10-30-2013

\title{
Target-Spin Asymmetry Measurements for Deeply Virtual Compton Scattering on Longitudinally Polarized Protons
}

Erin E. Seder

University of Connecticut - Storrs, seder@phys.uconn.edu

Follow this and additional works at: https://opencommons.uconn.edu/dissertations

\section{Recommended Citation}

Seder, Erin E., "Target-Spin Asymmetry Measurements for Deeply Virtual Compton Scattering on Longitudinally Polarized Protons" (2013). Doctoral Dissertations. 247.

https://opencommons.uconn.edu/dissertations/247 


\title{
Target-Spin Asymmetry Measurements for Deeply Virtual Compton Scattering on Longitudinally Polarized Protons
}

\author{
Erin E. Seder, Ph.D. \\ University of Connecticut, 2013
}

\begin{abstract}
This work studies the target-spin asymmetry from the EG1-DVCS experiment conducted in Hall B of Jefferson National Lab using the Continuous Electron Beam Accelerator Facility's $6 \mathrm{GeV}$ electron beam, a longitudinally polarized solid-state $\left({ }^{14} \mathrm{NH}_{3}\right)$ target, and the CEBAF Large Acceptance Spectrometer equipped with an additionally built Inner Calorimeter. The high statistics collected allow for detailed studies of the $Q^{2}, x_{B}$ and $\mathrm{t}$ dependencies of the DVCS amplitude over a wide range of kinematics. This measurement, in combination with the measurement of the beam spin asymmetry and double spin asymmetry allows for extraction of GPD related quantities, Compton Form Factors: $\Re$ e $\mathcal{H}, \Im \mathrm{m} \mathcal{H}, \Re \mathrm{e} \tilde{\mathcal{H}}$, эm $\tilde{\mathcal{H}}$, and $\Im \mathrm{mE}$.
\end{abstract}




\title{
Target-Spin Asymmetry Measurements for Deeply Virtual Compton Scattering on Longitudinally Polarized Protons
}

\author{
Erin E. Seder \\ B.S. Applied Physics, Richard Stockton College of NJ, Pomona, NJ, 2004 \\ M.S. Physics, University of Connecticut, Storrs, CT, 2011
}

\author{
A Dissertation \\ Submitted in Partial Fullfilment of the \\ Requirements for the Degree of \\ Doctor of Philosophy \\ at the \\ University of Connecticut
}


Copyright by

Erin E. Seder 


\section{APPROVAL PAGE}

Doctor of Philosophy Dissertation

\section{Target-Spin Asymmetry Measurements for Deeply Virtual Compton Scattering on Longitudinally Polarized Protons}

\begin{tabular}{lc} 
& \multicolumn{2}{c}{ Presented by } \\
& Erin E. Seder, B.S., M.S. \\
& \\
Major Advisor & Kyungseon Joo \\
\cline { 2 - 2 } Associate Advisor & \\
\cline { 2 - 2 } Associate Advisor & Andrew Puckett \\
\cline { 2 - 2 } Associate Advisor & Richard Jones \\
\cline { 2 - 2 } & \\
\hline
\end{tabular}

Peter Schweitzer

University of Connecticut

2013 


\section{TABLE OF CONTENTS}

1. Introduction . . . . . . . . . . . . . . . . . . . . 1

1.1 Deep Inelastic Scattering . . . . . . . . . . . . . . . . . . . . . . . . 1

1.2 Deep Exclusive Electroproduction of a Photon . . . . . . . . . . . . . . . . . . 2

1.3 Generalized Parton Distributions . . . . . . . . . . . . . . . . . . . . 4

2. Experimental Setup . . . . . . . . . . . . . . . . . . . 8

2.1 EG1-DVCS Experiment . . . . . . . . . . . . . . . . 8

2.2 Continuous Electron Beam Accelerator Facility . . . . . . . . . . . . . . . . 9

2.3 Experimental Hall B Beamline . . . . . . . . . . . . . . . . . . . . . . 13

2.4 Hall B CEBAF Large Acceptance Spectrometer . . . . . . . . . . . . . . . . . . 21

2.5 Data Processing . . . . . . . . . . . . . . . . . . . . . 32

2.6 Polarized Target . . . . . . . . . . . . . . . . . . . . . . . . 32

3. Data Selection . . . . . . . . . . . . . . . . . . . 50

3.1 Run Selection . . . . . . . . . . . . . . . . . . . 50

3.2 Particle Identification . . . . . . . . . . . . . . . . . . . . . . . . . . . . 52

3.3 Exclusive ep Elastic Selection . . . . . . . . . . . . . . . . . . 78

3.4 Monte-Carlo Simulations . . . . . . . . . . . . . . . . . . . . . . . . . 81

3.5 DVCS Channel Selection . . . . . . . . . . . . . . . . . . 93

3.6 Exclusive $e p \pi^{0}$ Selection . . . . . . . . . . . . . . . . . . . . . . . . . . . . . 110

3.7 Four-dimensional binning . . . . . . . . . . . . . . . . . . . 121

4. Data Analysis . . . . . . . . . . . . . . . . . . . . . . . . . . . . . 124

4.1 Electron Beam Energy . . . . . . . . . . . . . . . . . . . . . . . 124

4.2 Proton Polarization Using Elastic Asymmetry . . . . . . . . . . . . . . . . . . 127

4.3 Electron Beam Raster Correction . . . . . . . . . . . . . . . . . . . . . . . . . . 134

4.4 Angular Correction . . . . . . . . . . . . . . . . . . . . . 135

4.5 Momentum Correction . . . . . . . . . . . . . . . . . . . . . . . 138

4.6 Dilution Factor Correction . . . . . . . . . . . . . . . . . . . . 143 
$4.7 \pi^{0}$ Contamination $\ldots \ldots \ldots \ldots \ldots \ldots \ldots \ldots \ldots$

5. Results . . . . . . . . . . . . . . . . . . . . . 163

5.1 Target-Spin Asymmetry Definition _ . . . . . . . . . . . . . . . . . 163

5.2 Statistical Error . . . . . . . . . . . . . . . . . . . . . . . . . . . . . . 164

5.3 Target-Spin Asymmetry Results . . . . . . . . . . . . . . . . . . . . . . . . . 164

5.4 Combination of EG1-DVCS Run Periods A and B . . . . . . . . . . . . . 170

5.5 Merged Asymmetries . . . . . . . . . . . . . . . . . . . 175

5.6 Systematic Errors . . . . . . . . . . . . . . . . . . . . . . . . . . . 182

6. Physics Analysis: Extraction of Compton Form Factors . . . . . . . . . 194

6.1 Target-Spin Asymmetry . . . . . . . . . . . . . . . . . . . . . . . 195

6.2 Beam-Spin Asymmetry . . . . . . . . . . . . . . . . . . . . . . . 195

6.3 Double-Spin Asymmetry . . . . . . . . . . . . . . . . . . . . . 195

6.4 Extracted Compton Form Factors . . . . . . . . . . . . . . . . . 196

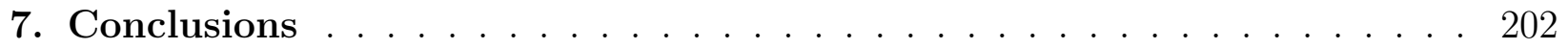

A. DVCS Channel Selection - Run Period A . . . . . . . . . . . . 203

B. Exclusive $e p \pi^{0}$ Selection - Run Period A . . . . . . . . . . . . . . . 212

C. $\pi^{0}$ Contamination - Run Period A . . . . . . . . . . . . 222

$\begin{array}{ll}\text { Bibliography } & 237\end{array}$ 


\section{Chapter 1}

\section{Introduction}

\section{$1.1 \quad$ Deep Inelastic Scattering}

High-energy lepton-nucleon scattering, also known as Deep Inelastic Scattering (DIS), has been utilized since the 1960's and continues to play a key role in determining the structure of nucleons. Using a lepton as a probe is advantageous because it interacts with the electromagnetic current density of the nucleon, described by QED. The coupling strength of the interaction is given by the fine structure constant $\alpha(\sim 1 / 137)$. The one photon exchange, the lowest order process, can be assumed in most analysis because an additional photon exchange would contribute to the cross section as $\alpha^{2}$ and can therefore be neglected. Scattering is considered deeply inelastic when the photon virtuality $\left(Q^{2}\right)$, the amount by which the photon is off it's mass shell, is high enough that the electron interacts via a virtual photon with a single quark of the nucleon (Figure 1.1) [2]. The signature of interaction with single quark is an observed $Q^{2}$ independence of the DIS scattering amplitude, known as "Bjorken scaling". The scaling regime has been observed experimentally to be valid for $Q^{2} \gtrsim 1 \mathrm{GeV}^{2}$.

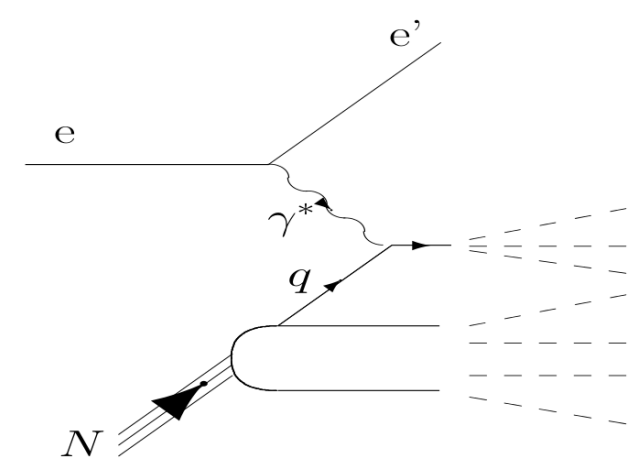

Fig. 1.1: Deep Inelastic Scattering [3]. 


\subsection{Deep Exclusive Electroproduction of a Photon}

For exclusive deep exclusive electroproduction of a real photon, the four-fold differential cross section is given by

$$
\frac{d \sigma}{d x_{B} d Q^{2} d|t| d \phi}=\frac{x_{B} e^{6}|\tau|^{2}}{32(2 \pi)^{2} Q^{4} \sqrt{1+\epsilon^{2}}}
$$

with

- $x_{B}$, the standard Bjorken variable $Q^{2} /(2 M \nu)(M=$ the mass of the target nucleon and $\nu=$ electron's energy loss in the nucleon rest frame).

- $Q^{2}$, photon virtuality, $Q^{2}=-q^{2}$ (with $q=k-k^{\prime}$, the 4-momentum of the virtual photon).

- $t=\left(p-p^{\prime}\right)^{2}$ (the squared 4-momentum transfer between the final and initial nucleon).

- $\epsilon=2 x_{B} M / Q$.

The scattering amplitude, $\tau$, can be written in terms of the amplitudes of two experimentally indistinguishable processes, shown schematically in Figure 1.2. The Bethe-Heitler (BH) process occurs when the incident or scattered electron emits a real photon, and Deeply Virtual Compton Scattering (DVCS) when the recoiling nucleon emits a real photon. Equation 1.2 shows the scattering amplitude expressed in terms of the BH and DVCS amplitudes.

$$
|\tau|^{2}=\left|\tau_{D V C S}\right|^{2}+\left|\tau_{B H}\right|^{2}+\left\{\tau_{D V C S} \tau_{B H}^{*}+\tau_{D V C S}^{*} \tau_{B H}\right\}
$$

To leading order in the electromagnetic coupling constant $\alpha$, the Fourier expansion of Equation 1.2 for a charged lepton beam and a target nucleon, where both the beam and the target are longitudinally polarised reads [5]:

$$
\begin{array}{r}
\left|\tau_{B H}\right|^{2}=\frac{K_{\mathcal{B H}}}{\mathcal{P}_{1}(\phi) \mathcal{P}_{2}(\phi)}\left\{\sum_{n=0}^{2} c_{n, u n p}^{\mathcal{B H}} \cos (n \phi)+P_{l} P_{t} \sum_{n=0}^{1} c_{n, L P}^{\mathcal{B H}} \cos (n \phi)\right\} \\
\left|\tau_{D V C S}\right|^{2}=K_{\mathcal{D V} \mathcal{C S}}\left\{\sum_{n=0}^{2} c_{n, u n p}^{\mathcal{D V} \mathcal{C} \mathcal{S}} \cos (n \phi)+P_{l} s_{1, u n p}^{\mathcal{D V} \mathcal{C}} \sin (\phi)\right. \\
\left.+P_{t}\left[P_{l} \sum_{n=0}^{1} c_{n, L P}^{\mathcal{D V C S}} \cos (n \phi)+\sum_{n=1}^{2} s_{n, L P}^{\mathcal{D V C S}} \sin (n \phi)\right]\right\}
\end{array}
$$




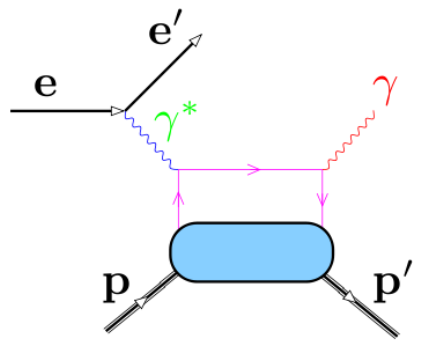

(a)

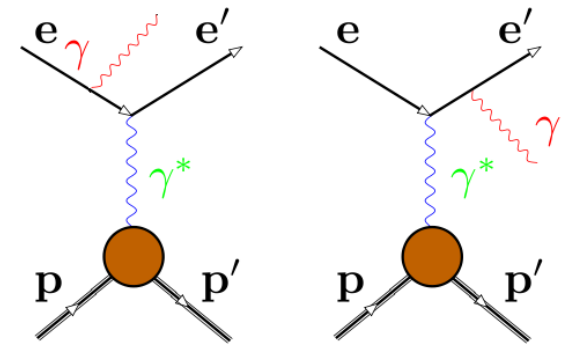

(b)

Fig. 1.2: (a) Diagram for Deeply Virtual Compton Scattering and (b) Bethe-Heitler electroproduction processes.

$$
\begin{array}{r}
\left\{\tau_{D V C S} \tau_{B H}^{*}+\tau_{D V C S}^{*} \tau_{B H}\right\}=\frac{-e K_{\mathcal{I}}}{\mathcal{P}_{1}(\phi) \mathcal{P}_{2}(\phi)}\left\{\sum_{n=0}^{3} c_{n, u n p}^{\mathcal{I}} \cos (n \phi)+P_{l} \sum_{n=1}^{2} s_{n, u n p}^{\mathcal{I}} \sin (n \phi)\right. \\
\left.+P_{t}\left[P_{l} \sum_{n=0}^{2} c_{n, L P}^{\mathcal{I}} \cos (n \phi)+\sum_{n=1}^{3} s_{n, L P}^{\mathcal{I}} \sin (n \phi)\right]\right\}
\end{array}
$$

The focus of the work presented in this thesis is to measure the target longitudinal spin asymmetry, $A_{U L}$, which is defined as the cross section difference between the 2 nucleon spin states divided by the total cross section (Equation 1.6). For the $A_{U L}$ measurement, the electron polarization, $\uparrow \downarrow$, is integrated over and only the target polarization, $\Uparrow \downarrow$, is considered.

$$
\begin{aligned}
& A_{U L}(\phi)=\frac{\left\{\sigma^{\Uparrow \Uparrow+\sigma}+\sigma^{\downarrow \Uparrow}\right\}-\left\{\sigma^{\Uparrow \Downarrow}+\sigma \downarrow \Downarrow\right\}}{\{\sigma \Uparrow \Uparrow+\sigma \downarrow \Uparrow\}+\left\{\sigma^{\top \Downarrow}+\sigma \downarrow \Downarrow\right\}}
\end{aligned}
$$

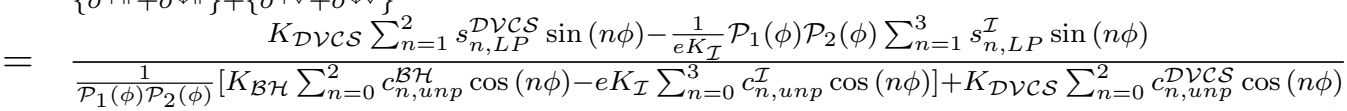

In Equations 1.3, 1.5, and 1.6, $\mathcal{P}_{1}(\phi)$ and $\mathcal{P}_{2}(\phi)$ are lepton Bethe-Heitler propagators, defined as:

$$
\begin{aligned}
& Q^{2} \mathcal{P}_{1}=\left(k-q_{2}\right)^{2} \\
& Q^{2} \mathcal{P}_{2}=\left(k-\left(p^{\prime}-p\right)\right)^{2}
\end{aligned}
$$

where $k, p, p^{\prime}$ and $q_{2}$ are momenta of the incoming electron, proton and outgoing proton and real photon respectively. The terms $P_{l}$ and $P_{t}$ are the linear polarization of the electron beam and target respectively. $K_{B H}, K_{D V C S}, K_{I}$ are kinematic factors:

$$
\begin{aligned}
K_{\mathcal{B H}} & =\frac{1}{x_{B}^{2} t\left(1+\epsilon^{2}\right)^{2}} \\
K_{\mathcal{D V C S}} & =\frac{1}{Q^{2}} \\
K_{\mathcal{I}} & =\frac{1}{x_{B} y t}
\end{aligned}
$$


where the fractional energy loss of the lepton, $y=\left(E-E^{\prime}\right) / E$. The BH coefficients, $c_{n}^{\mathcal{B H}}$, are exactly calculable in leading-order QED using the known elastic nucleon Dirac and Pauli

form factors $F_{1}$ and $F_{2}$ respectively. The DVCS coefficients, $c_{n}^{\mathcal{D V C S}}$ and $s_{n}^{\mathcal{D V C S}}$ relate to a bilinear combination of Generalized Parton Distributions (GPDs), whereas the coefficients $c_{n}^{\mathcal{I}}$ and $s_{n}^{\mathcal{I}}$ relate to linear combinations of GPDs. A full description of the relation of the DVCS coefficients to GPDs can be found in Reference [5].

\subsection{Generalized Parton Distributions}

Generalized Parton Distributions (GPDs) are a tool for studying qualitatively new aspects of hadron structure. GPDs encompass the familiar Parton Distribution Functions (PDFs) and nucleon Form Factors (FFs) to provide a comprehensive description of the structure of the nucleon. They correspond to the Fourier transform of the QCD non-local (initial and final quarks are created or annihilated at different space-time points), non-diagonal (momenta of initial and final nucleons are different) operators $\left\langle p^{\prime}\left|\bar{\Psi}_{q}(0) \mathcal{O} \Psi_{q}(y)\right|\right\rangle$ where $\mathcal{O}=\gamma^{+}$or $\gamma^{+} \gamma^{5}$. Original articles and general reviews on GPDs and details on the formalism can be found in References [6-12]. A thorough description of the nucleon in terms of GPDs would allow the deduction of the total angular momentum of partons in the nucleon, and the construction of a longitudinal-momentum-dissected transverse spatial map of parton densities [13]. As mentioned in the above section, the coefficients in the interference term of the DVCS amplitude, Equation 1.5, relate to linear combinations of GPDs making DVCS an ideal means for accessing GPDs.

In the QCD leading twist and leading order approximation there are 4 independent GPDs, H, E, $\tilde{\mathbf{H}}, \tilde{\mathbf{E}}$ that correspond to different spin and helicity orientations of the quark and nucleon, as illustrated in Figure 1.3.

Figure 1.4 depicts the dominant mechanism of the DVCS process known as the Handbag diagram. In the Handbag approach DVCS can be factorized into a hard scattering part, calculable in pQCD, and a nonperturbative part described by the above mentioned GPDs.

These GPDs depend on 3 variables, $x, \xi$ and $t . \xi$ and $x$ express the longitudinal momentum fractions, with $x+\xi(x-\xi)$ being the the fraction of the nucleon's momentum 

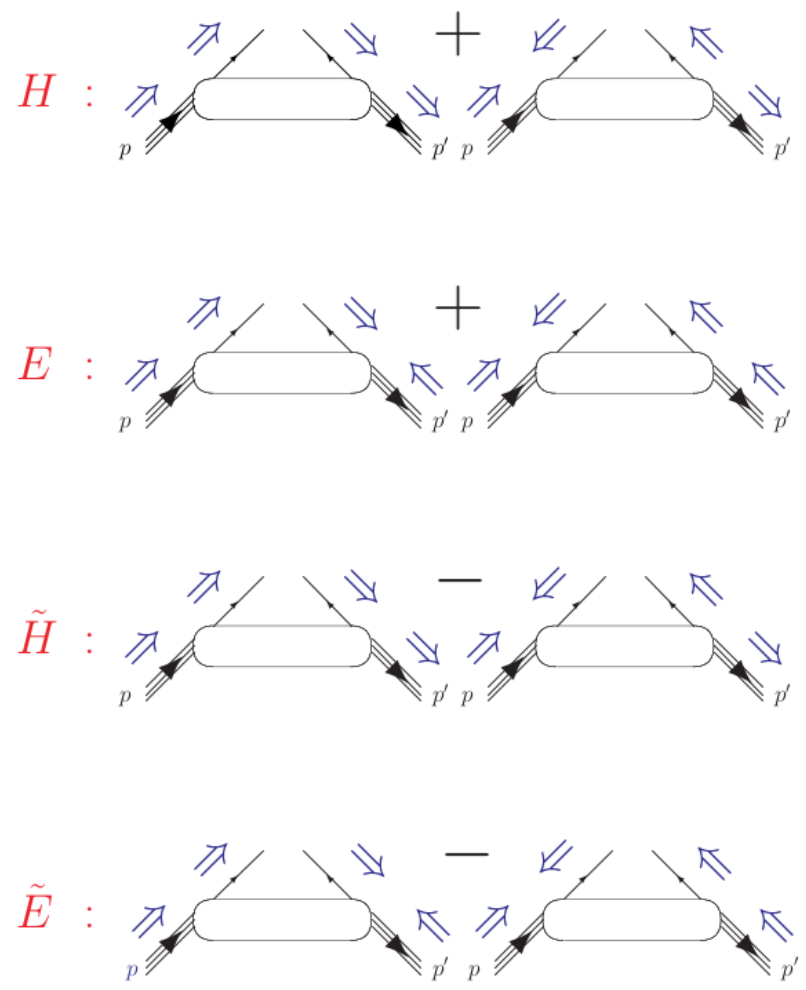

Fig. 1.3: The four GPDs $\mathbf{H}, \mathbf{E}, \tilde{\mathbf{H}}, \tilde{\mathbf{E}}$ correspondence to the various quarks helicity and nucleon spin orientations $(\Uparrow \Downarrow)[3]$.

carried by the initial (final) quark. In the Bjorken limit of photon virtuality $Q^{2} \rightarrow \infty$ with fixed $t, \xi \simeq \frac{x_{B} / 2}{1-x_{B} / 2}$, where $x_{B}$ is the standard Bjorken variable. $t$ is the squared 4-momentum transfer between the final and initial nucleon. The first moments of the leading twist GPDs are equal to the Dirac, Pauli, axial, and pseudoscalar parton form factors in the nucleon as [4]:

$$
\begin{aligned}
\int_{-1}^{1} \mathbf{H}(x, \xi, t), \mathrm{d} x & =F_{1}(t) \\
\int_{-1}^{1} \tilde{\mathbf{H}}(x, \xi, t), \mathrm{d} x & =G_{1}(t) \\
\int_{-1}^{1} \mathbf{E}(x, \xi, t), \mathrm{d} x & =F_{2}(t) \\
\int_{-1}^{1} \tilde{\mathbf{E}}(x, \xi, t), \mathrm{d} x & =G_{p}(t)
\end{aligned}
$$

and in the forward direction, i.e. $\xi=t=0$, they relate to Parton Distribution Functions 


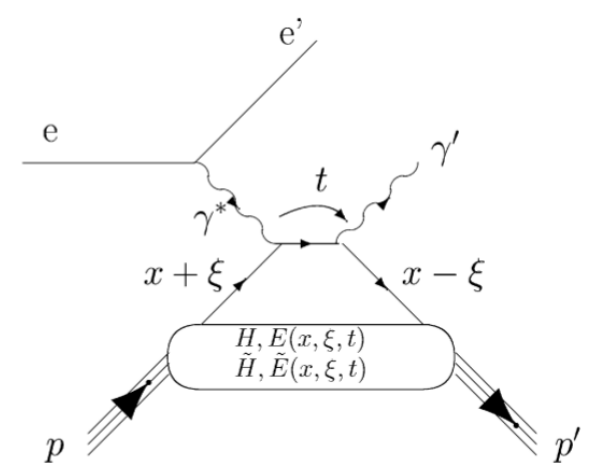

Fig. 1.4: Leading order Handbag diagram for Deeply Virtual Compton Scattering on the proton [3].

(PDFs) as [4]:

$$
\begin{aligned}
\mathbf{H}^{q}(x, 0,0) & =\left\{\begin{array}{cc}
q(x), & x>0, \\
-\bar{q}(-x), & x<0 .
\end{array}\right. \\
\tilde{\mathbf{H}}^{q}(x, 0,0) & =\left\{\begin{array}{cc}
\Delta q(x), & x>0, \\
-\Delta \bar{q}(-x), & x<0 .
\end{array}\right.
\end{aligned}
$$

Although GPDs are a function of 3 variables, they enter into the DVCS amplitude within a convolution integral over $x$, i.e. $\int_{-1}^{+1} \frac{\mathbf{H}(x, \xi, t)}{x \pm \xi+i \epsilon}, \mathrm{d} x$. Following reference [3], decomposing that integral, as well as the similar ones for $\mathbf{E}, \tilde{\mathbf{H}}, \tilde{\mathbf{E}}$ into their real and imaginary parts and reducing the $\mathrm{x}$-range of integration from $\{-1,1\}$ to $\{0,1\}$ in the convolutions leads to eight GPD related quantities accessible through DVCS observables, called Compton Form Factors (CFFs) as given in Equations 1.11 where $P$ denotes the principal value integral.

$$
\begin{aligned}
\Re \mathrm{e} \mathcal{H} & =P \int_{0}^{+1} d x\left(\frac{1}{x-\xi}+\frac{1}{x+\xi}\right)[\mathbf{H}(x, \xi, t)-\mathbf{H}(-x, \xi, t)] \\
\Im \mathrm{m} \mathcal{H} & =[\mathbf{H}(\xi, \xi, t)-\mathbf{H}(-\xi, \xi, t)] \\
\Re \mathrm{e} \tilde{\mathcal{H}} & =P \int_{0}^{+1} d x\left(\frac{1}{x-\xi}-\frac{1}{x+\xi}\right)[\tilde{\mathbf{H}}(x, \xi, t)+\tilde{\mathbf{H}}(-x, \xi, t)] \\
\Im \mathrm{m} \tilde{\mathcal{H}} & =[\tilde{\mathbf{H}}(\xi, \xi, t)+\tilde{\mathbf{H}}(-\xi, \xi, t)] \\
\Re \mathrm{E} & =P \int_{0}^{+1} d x\left(\frac{1}{x-\xi}+\frac{1}{x+\xi}\right)[\mathbf{E}(x, \xi, t)-\mathbf{E}(x, \xi, t)] \\
\Im \mathrm{m \mathcal {E }} & =[\mathbf{E}(\xi, \xi, t)-\mathbf{E}(-\xi, \xi, t)] \\
\Re \mathrm{E} \tilde{\mathcal{E}} & =P \int_{0}^{+1} d x\left(\frac{1}{x-\xi}-\frac{1}{x+\xi}\right)[\tilde{\mathbf{E}}(x, \xi, t)+\tilde{\mathbf{E}}(x, \xi, t)] \\
\Im \mathrm{m} \tilde{\mathcal{E}} & =[\tilde{\mathbf{E}}(\xi, \xi, t)+\tilde{\mathbf{E}}(-\xi, \xi, t)]
\end{aligned}
$$

Different DVCS observables are sensitive to different terms in 1.11 with the maximum 
information that can be extracted from the experimental data at a given $(\xi, t)$ point being $H( \pm \xi, \xi, t)$, when measuring an observable sensitive to the imaginary part of the DVCS amplitude, and $\int_{-1}^{+1} d x \frac{H(\mp x, \xi, t)}{x \pm \xi}$, when measuring an observable sensitive to the real part of the DVCS amplitude.

By using the information of different DVCS observables within the same kinematics it is possible to constrain the eight GPD related quantities in a model independent fashion, as is discussed in Section 6.

JLab has provided the first DVCS measurement, in the valence region, of beampolarized and unpolarized DVCS cross sections, in a limited phase-space domain, with the Hall A spectrometer [14], and beam-spin asymmetries $\left(A_{\mathrm{LU}}\right)$, over a large kinematic range, obtained with the CLAS detector [15]. Beam-charge asymmetries $\left(A_{\mathrm{C}}\right)$, longitudinally and transversely-polarized target-spin asymmetries, $A_{\mathrm{UL}}$, and $A_{\mathrm{UT}}$, as well as doublespin asymmetries, $A_{\mathrm{LL}}$, have also been measured by the HERMES collaboration [16]. $\mathrm{A}$ measurement of target-spin asymmetry $\left(A_{\mathrm{UL}}\right)$ analyzing non-dedicated CLAS data on a longitudinally polarized target was also performed, with limited statistics [17].

The work of this thesis is to measure the single target-spin asymmetry, $A_{U L}$, over a large kinematic range for the DVCS+BH process from a dedicated DVCS experiment, EG1-DVCS, conducted in 2009 using a polarized electron beam of $6 \mathrm{GeV}$ incident on a longitudinally polarized proton target. The experiment took place in Hall B of Jefferson National Lab using the CEBAF Large Acceptance Spectrometer (CLAS) equipped with an additionally built Inner Calorimeter for improved DVCS statistics. This measurement was combined with the measurement of the beam spin asymmetry, $A_{\mathrm{LU}}$, and double spin asymmetry, $A_{\mathrm{LL}}$, (measured by S. Pisano [1]) for extraction of the Compton Form Factors: $\Re \mathrm{H}, \Im \mathrm{m} \mathcal{H}, \Re \mathrm{e} \tilde{\mathcal{H}}, \Im \mathrm{m} \tilde{\mathcal{H}}$, and $\Im \mathrm{m} \mathcal{E}$. The following chapters discuss the experimental setup, data analysis, and results associated with this measurement. 


\section{Chapter 2}

\section{Experimental Setup}

\subsection{EG1-DVCS Experiment}

The data presented in this thesis come from the EG1-DVCS experiment, which ran in 3 installments (A, B, C) from February to September 2009. The experiment used Jefferson National Lab's Continuous Electron Beam Accelerator Facility's (CEBAF) $6 \mathrm{GeV}$ polarized electron beam and the experimental Hall-B's CEBAF Large Acceptance Spectrometer (CLAS) with an additional Inner Calorimeter. The experiment implemented a polarized target: polarized protons for Periods A and B, and polarized deuterons for Period C. Period $\mathrm{C}$ is not included in this analysis. Periods $\mathrm{A}$ and $\mathrm{B}$ mainly differ in three aspects. The first is in the target position with respect to the center of CLAS (see Figure 2.21 for a definition of CLAS center). For Period A, the target is $57.95 \mathrm{~cm}$ upstream of CLAS center and for Period B the target is $67.97 \mathrm{~cm}$ upstream of CLAS center. This difference slightly affects the angular coverage of CLAS (Section 2.4) and the Inner Calorimeter (Section 2.4.0.6). The second difference is in accumulated charge, shown in Figure 2.1. Period A ran for 31 days with 6.9 $\mathrm{mC}$ accumulated charge and 5,642 million events triggered. Period B ran substantially longer, 51 days, accumulating $15.4 \mathrm{mC}$ of charge with 14,149 million events triggered. The third difference is in the electron beam's average energy and polarization, discussed in Section 2.2).

The following sections give an overview of the experimental equipment, including the Continuous Electron Beam Accelerator Facility (CEBAF), the CEBAF Large Acceptance Spectrometer (CLAS), and the Polarized Target System used in the EG1-DVCS experiment. 

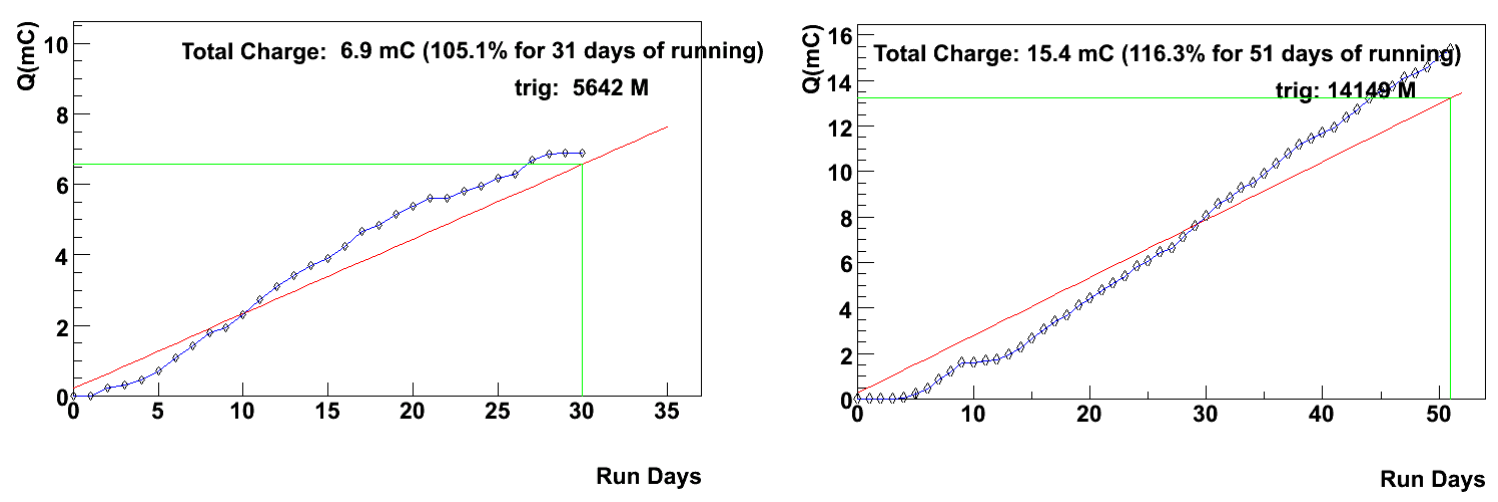

Fig. 2.1: Accumulated charge plotted as a function of time for EG1-DVCS Run Period A (left) and Run Period B (right). The red line indicated the projected accumulation, the black circles the measured accumulation.

\subsection{Continuous Electron Beam Accelerator Facility}

The Continuous Electron Beam Accelerator Facility (CEBAF) produces high power electron and photon beams with energies up to $6 \mathrm{GeV}$ and $100 \%$ duty cycle [18]. A drawing of the accelerator and experimental halls is shown in Figure 2.2. The accelerator consists of an injector, two anti-parallel superconducting linac segments connected by nine recirculation arcs, and a beam switch yard. The source of the polarized electrons is a $100 \mathrm{keV}$ electron gun which produces $45 \mathrm{MeV}$ electrons with a polarization of $80 \%$ or greater. The process in simplicity involves shining a $500 \mathrm{~mW}$ Ti-sapphire laser, circularly polarized, on a gallium arsenide (GaAs) cathode, exciting the $P_{1 / 2}$ and $P_{3 / 2}$ state electrons up to the conduction band. The high polarization is achieved by using a $G a A s$ lattice grown on a $G a A s_{0.72} P_{0.28}$ substrate. This creates a strain on the GaAs lattice due to the smaller spacing of the substrate thereby separating the otherwise degenerate $\mathrm{m}=1 / 2$ and $\mathrm{m}=3 / 2$ states for the $P_{3 / 2}$ state electrons, Figure 2.3. The helicity of the electron beam is flipped at a frequency on the order of $\mathrm{Hz}$ by inverting the laser polarization, and also on a longer timescale by periodically inserting a half-wave plate. Implementing both methods serves to reduce the systematics associated with beam differences between the two helicity sates.

The electron gun is followed by a prebuncher cavity and two circular apertures which 


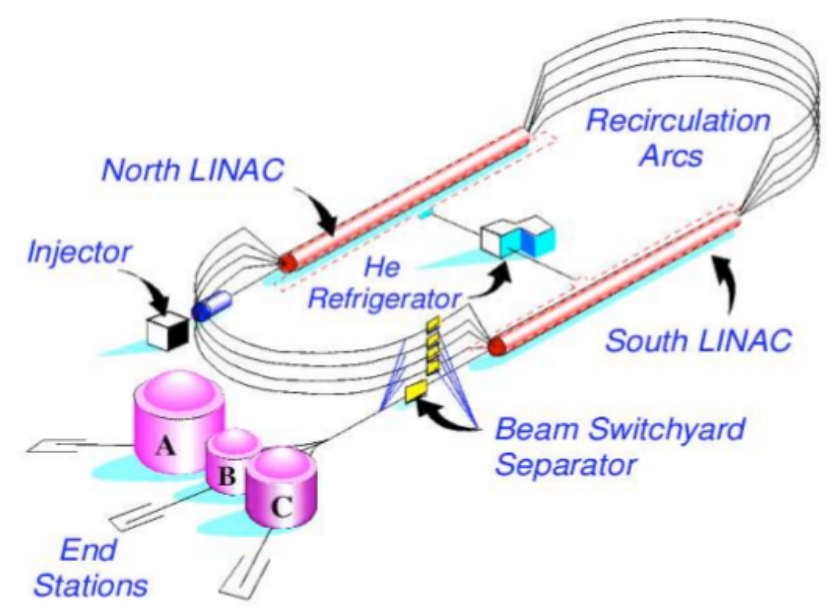

Fig. 2.2: The Continuous Electron Beam Accelerator Facility (CEBAF) at Jefferson National Lab [18]

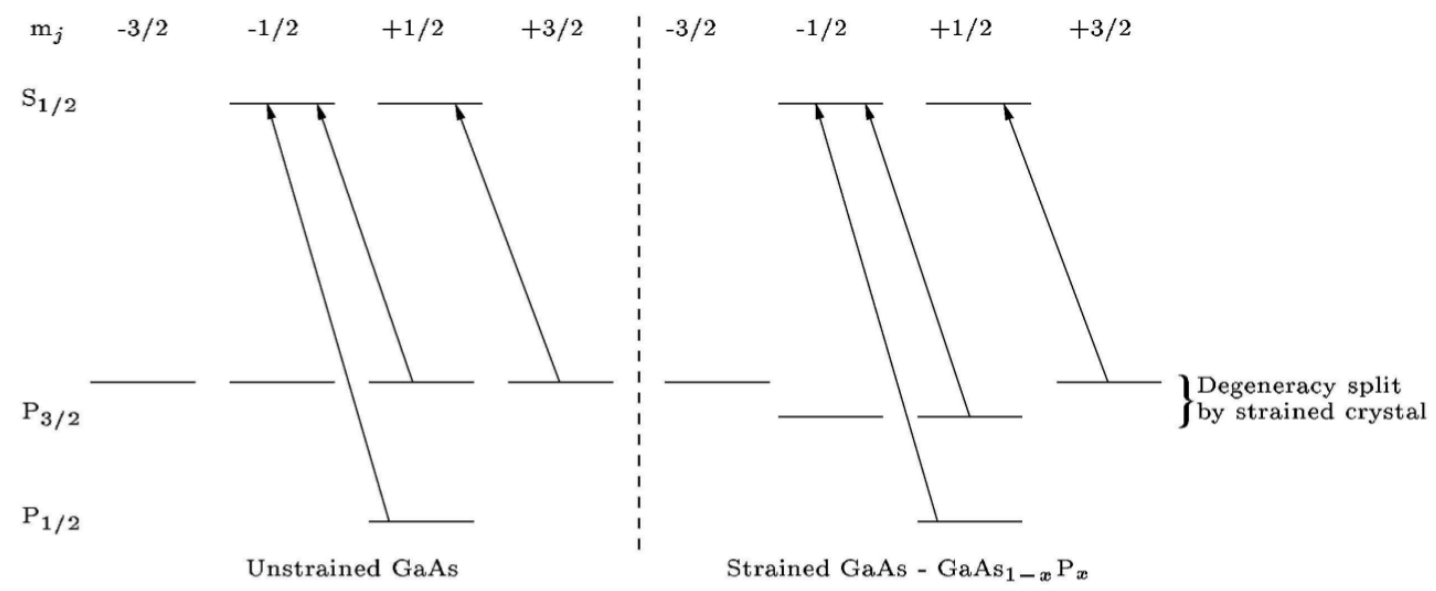

Fig. 2.3: The electron energy levels for $G a A s$ (left) and $G a A s-G a A s_{1-x} P_{x}$ (right) [24]. 
pare down the beam in length and diameter so that it can be passed to the chopper, which splits the beam into bunches. After the chopper, the beam enters a buncher cavity which compresses the bunches and then to a room temperature capture region with $2 \mathrm{RF}$ amplifiers increasing the beam energy to $540 \mathrm{keV}$. The capture region is followed by 2 superconducting RF cavities producing a beam of $5.15 \mathrm{MeV}$. The beam then passes through a tuning region and onto two cryomodules, each with 8 superconducting RF cavities, increasing the energy up to about $45 \mathrm{MeV}$ for injection into the north linac [25].

Once the beam exits the injector it can make up to 5 passes through the two linacs, labeled north and south, via the recirculation arcs (Figure 2.2). Each linac spans approximately 240 meters and consists of 25 cryomodules, each with 8 superconducting niobium RF cavities. After being accelerated in one of the linac segments the electron beam is fed into and out of the recirculation arcs by transport lines consisting of spreaders, recirculation arcs, and recombiners. Optically similar spreaders and recombiners enable beams of different energy to transit the individually energy-tuned recirculation arcs. After traversing one of the linacs the beam is fed through spreaders that bend the beam vertically in a dipole through an angle $\alpha$, inversely proportional to the beam energy. After traveling through a recirculation arc, the recombiner, a mirror image of the spreader, bends the individual beams by an angle $-\alpha$ from the arc lines and feeds to the next linac. A maximum electron beam energy of approximately $6 \mathrm{GeV}$ is achieved after all 5 passes through the linacs.

The beam is extracted at the end of the south linac. As mentioned above, the beam is structured in microbunches which are separated by a 1/3-harmonic rf separator system and delivered in sequence to the three different experimental areas, Halls A, B, and C for simultaneous experiments. The microbunches for each Hall can be taken from any of the five-pass energies.

During the recirculation of the electron beam, the electron spin orientation undergoes a precession due to the magnetic field of the bending magnets. This is corrected by the use of a Wien filter whose perpendicular magnetic field induces a precession while its perpendicular electric field preserves the electron momentum by compensating for the deflection caused by the magnetic field. The total post accelerator precession angle is a function of the injector energy, the number of passes, and the linac energy. An Experimental Hall's optimal Wien 
angle for a particular beam condition can be seen through a "spin dance", where the Wien filter is rotated through several angles, with Møller measurements (see Section 2.3.1) taken at each angle. An example of the results of a spin dance for Hall-B is shown in Figure 2.4. Because the 3 Halls can be run at different energies the ideal Wien angle for one Hall is not necessarily the same for the other two. Maximum polarization can be obtained simultaneously for the 3 experimental Halls by choosing particular combinations of beam energy [26].

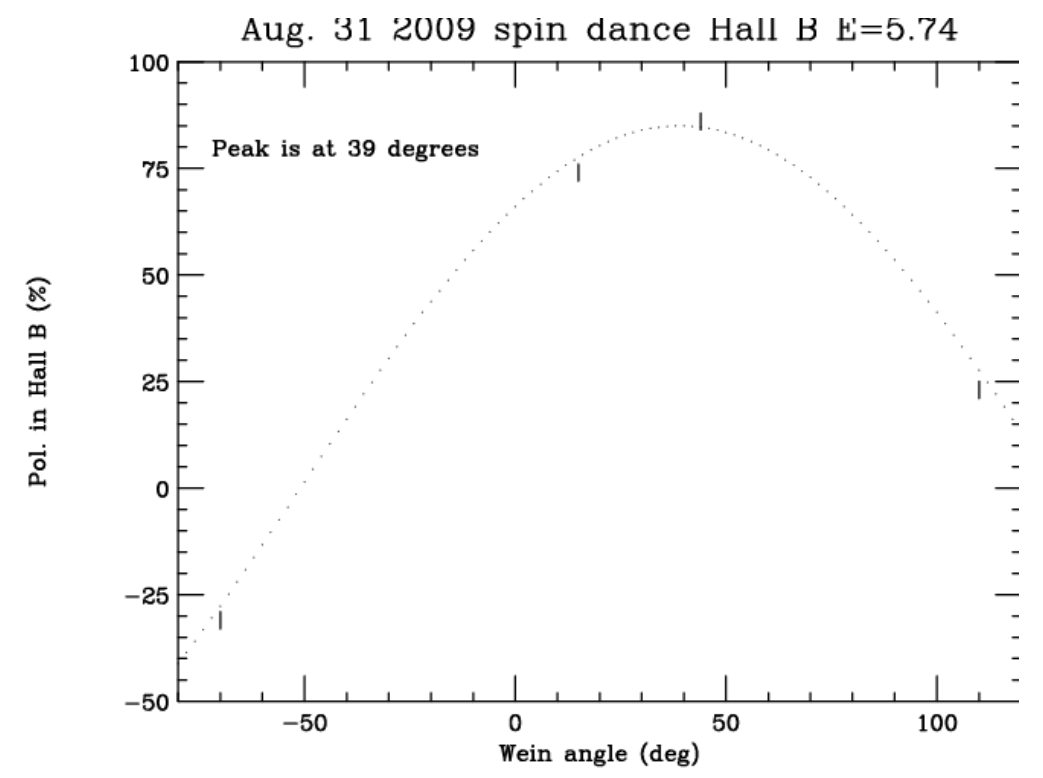

Fig. 2.4: Hall-B electron beam polarization results from a spin dance [27]. 


\subsection{Experimental Hall B Beamline}

The specifications of the electron beam delivered to Experimental Hall B are listed in Table 2.1. The following section provides an overview of the Hall B beam line systems.

\begin{tabular}{|l|l|l|}
\multicolumn{1}{c}{ Quantity } & \multicolumn{1}{c}{ Range } & \multicolumn{1}{c}{ Precision } \\
\hline \hline Energy & $0.8-6.0 \mathrm{Gev}$ & $\delta \mathrm{E} / \mathrm{E}<0.1 \%$ \\
Polarization & $40-85 \%$ & $\delta \mathrm{P} / \mathrm{P}<3 \%$ \\
Position & $\pm 3 \mathrm{~mm}$ off target center & $100 \mu \mathrm{m}$ \\
Width & $\sigma<250 \mu \mathrm{m}$ & $10 \mu \mathrm{m}$ \\
Beam halo & $1: 100,000$ & \\
Current & $1-30 \mathrm{nA}$ & $<1 \%$ \\
\hline \hline
\end{tabular}

Table 2.1: Summary of the Hall B electron beam properties

\subsubsection{Electron Beam Polarization Monitor}

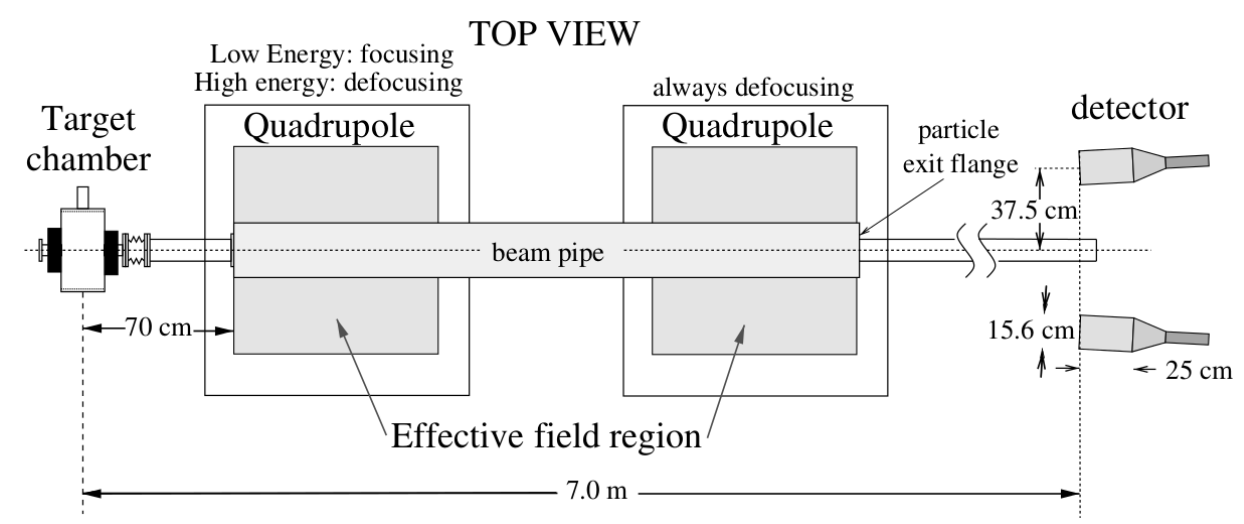

Fig. 2.5: Layout of the Hall B Møller polarimeter [18].

The electron polarization is measured by a Møller polarimeter, shown in Figure 2.5, located upstream of the target. The Møller target consists of a $25 \mu \mathrm{m}$ iron foil that can be polarized either parallel or antiparallel to the beam direction. This is done using a magnet in a Helmholtz configuration placed in the beam line. Electrons that scatter off 
the foil's electrons, and the recoil electrons, are deflected from the electron beam by two quadrupole magnets located downstream to the Møller target and detected in coincidence by two scintillator counters located symmetrically on the two sides of the beam line. The electron beam helicity asymmetry of Møller scattering, A, is a well know function of incoming and target electron polarization ( $P$ and $P^{T}$ respectively) and scattering angle $(\theta)$, and is given in Equation 2.1. The $z$ direction in Equation 2.1 is along the beam direction, and the $y$ direction is chosen so that the Møller target polarization is in the $y-z$ plane. Comparing the measured asymmetry to that of the theoretical value gives the electron beam polarization. Both polarization states of the electron beam are measured in sequence by reversing the Helmholtz current between two Møller runs. These measurements, which take about 30 minutes each, were taken periodically throughout the EG1-DVCS experiment, the results of which are listed in Tables 2.2 and 2.3.

$$
A=\frac{\sin ^{2}(\theta)}{\left(4-\sin ^{2}(\theta)\right)^{2}}\left(\sin ^{2}(\theta) P_{y} P_{y}^{T}-\left(8-\sin ^{2}(\theta)\right) P_{z} P_{z}^{T}\right.
$$

\begin{tabular}{c|c|c|c|}
$\begin{array}{c}\text { Date } \\
\text { Wien } \\
(\mathrm{mm} / \mathrm{dd} / \mathrm{yy})\end{array}$ & \multicolumn{1}{c}{ angle $\left(^{\circ}\right)$} & \multicolumn{1}{c}{$P+(\%)$} & $P-(\%)$ \\
\hline \hline $02 / 08 / 09$ & 29.99 & $88.70 \pm 1.48$ & $-81.09 \pm 1.48$ \\
$02 / 11 / 09$ & 29.99 & $91.12 \pm 1.05$ & $-83.78 \pm 1.05$ \\
$02 / 18 / 09$ & 22.99 & $90.82 \pm 1.48$ & $-87.04 \pm 1.48$ \\
$02 / 23 / 09$ & 12.99 & $89.64 \pm 1.00$ & $-84.14 \pm 1.00$ \\
$02 / 27 / 09$ & 29.99 & $90.64 \pm 1.45$ & $-79.09 \pm 1.48$ \\
$03 / 06 / 09$ & -17.99 & $75.19 \pm 1.49$ & $-68.01 \pm 1.49$ \\
$03 / 12 / 09$ & 21.19 & $90.60 \pm 1.32$ & $-84.25 \pm 1.42$ \\
\hline \hline
\end{tabular}

Table 2.2: Summary of the Hall B electron beam polarization measurements during EG1DVCS Run Period A. The errors listed are statistical. 
Date Wien

\begin{tabular}{c|c|c|c|}
$(\mathrm{mm} / \mathrm{dd} / \mathrm{yy})$ & \multicolumn{1}{c}{ angle $\left(^{\circ}\right)$} & $P+(\%)$ & $P-(\%)$ \\
\hline \hline $04 / 30 / 09$ & 74.83 & $87.53 \pm 1.54$ & $-81.44 \pm 1.52$ \\
$05 / 06 / 09$ & 74.83 & $81.42 \pm 1.33$ & $-82.14 \pm 1.61$ \\
$05 / 08 / 09$ & 66.92 & $86.13 \pm 1.48$ & $-84.71 \pm 1.51$ \\
$05 / 15 / 09$ & 66.92 & $89.93 \pm 1.34$ & $-80.11 \pm 1.45$ \\
$05 / 20 / 09$ & 66.92 & $81.97 \pm 1.44$ & $-86.51 \pm 1.14$ \\
$05 / 21 / 09$ & 66.92 & $81.55 \pm 1.44$ & $-80.25 \pm 1.38$ \\
$05 / 28 / 09$ & 66.92 & $85.72 \pm 1.50$ & $-81.80 \pm 1.03$ \\
$05 / 29 / 09$ & 66.92 & $84.57 \pm 1.49$ & $-82.68 \pm 1.48$ \\
$06 / 01 / 09$ & 66.92 & $81.20 \pm 1.48$ & $-85.20 \pm 1.05$ \\
$06 / 04 / 09$ & 66.92 & $88.55 \pm 1.28$ & $-75.55 \pm 0.95$ \\
$06 / 11 / 09$ & 66.92 & $85.15 \pm 1.48$ & $-83.99 \pm 1.48$ \\
$06 / 12 / 09$ & 66.92 & $85.85 \pm 1.49$ & $-85.28 \pm 1.48$ \\
\hline \hline
\end{tabular}

Table 2.3: Summary of the Hall B electron beam polarization measurements during EG1DVCS Run Period B. The errors listed are statistical. 


\subsubsection{Electron Beam Position and Current Monitor}

The electron beam position is measured continuously by three beam-position monitors (BPMs). Each of the three BPMs consists of three RF cavities. The three BPMs are located $36.0 m, 24.6 m$, and 8,2 $m$ upstream of CLAS center. These BPMs provide beam position coordinates $(\mathrm{x}, \mathrm{y})$ and beam intensity at each BPM location. The positional information is continuously used in feedback loops to keep the beam centered. Plots of the beam current and beam position during a 40 minute period is shown in Figure 2.6. The relative position calibration of the RF cavities is done with a cross calibration with the beam profile monitors (Section 2.3.3). Absolute position calibration is obtained by centering the electron beam in the upstream beamline quadrupoles, which have been surveyed into place. The current calibration is obtained through comparison with the calibrated Faraday cup (Section 2.3.5). 


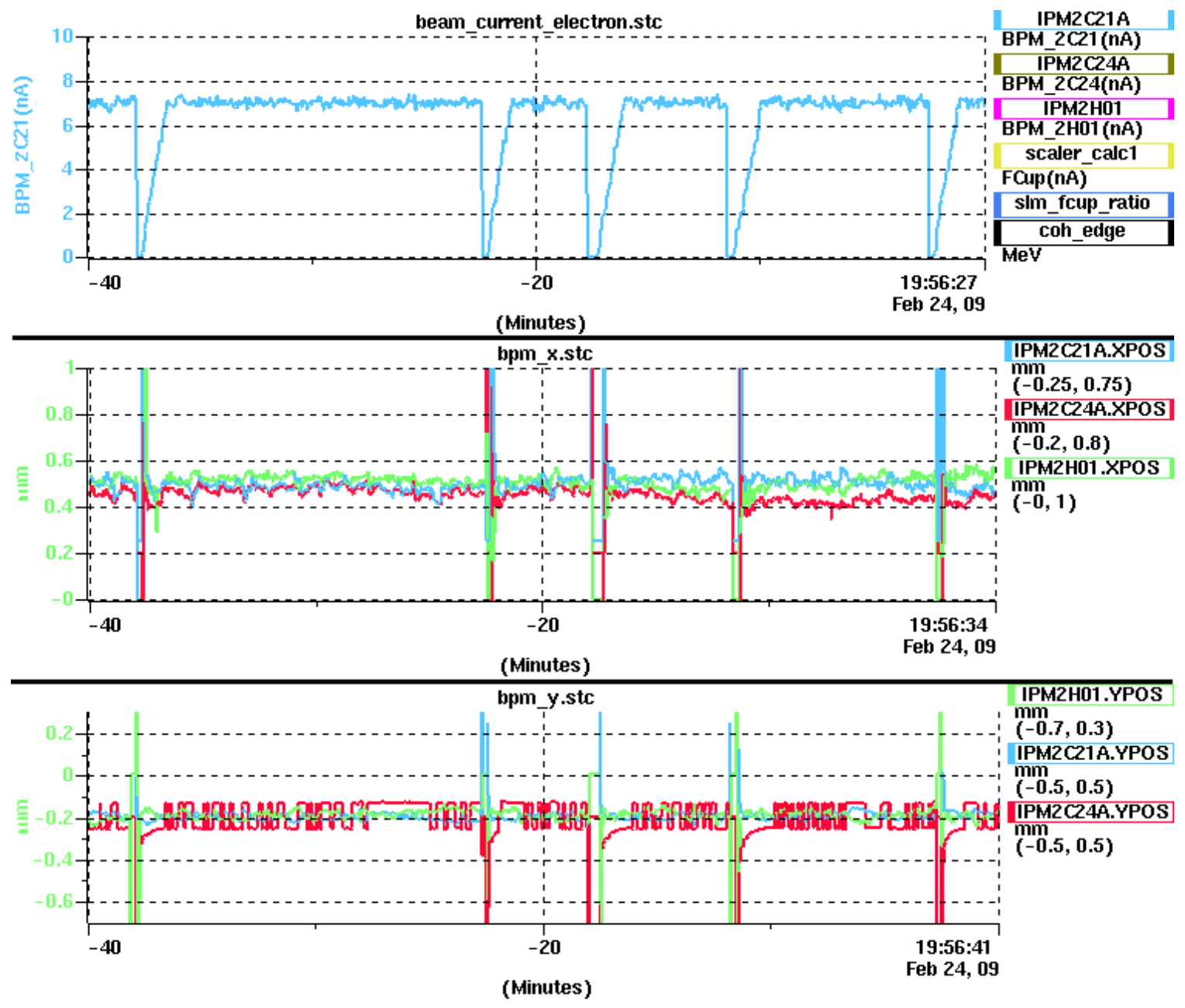

Fig. 2.6: Electron beam current and position (x,y) from BPM measurements during the EG1-DVCS experiment. 


\subsubsection{Electron Beam Profile Monitor}

The electron beam profile is measured by moving thin wires $(20 \mu m$ and $50 \mu m$ tungsten and $100 \mu \mathrm{m}$ iron) through the beam and detecting the scattered electrons via Cerenkov light in the glass windows of photo multiplier tubes. The wires are oriented along the horizontal and vertical axes with the direction of motion at $45^{\circ}$ with respect to the horizontal axis. The wire-moving device is called a harp. There are three harps located at $36.7 \mathrm{~m}, 22.1 \mathrm{~m}$, and $15.5 \mathrm{~m}$ upstream of the CLAS target. Harp scans are typically performed after any major change to the electron beam delivery. One of the EG1-DVCS experiment's beam profile from a harp scan is shown in Figure 2.7.
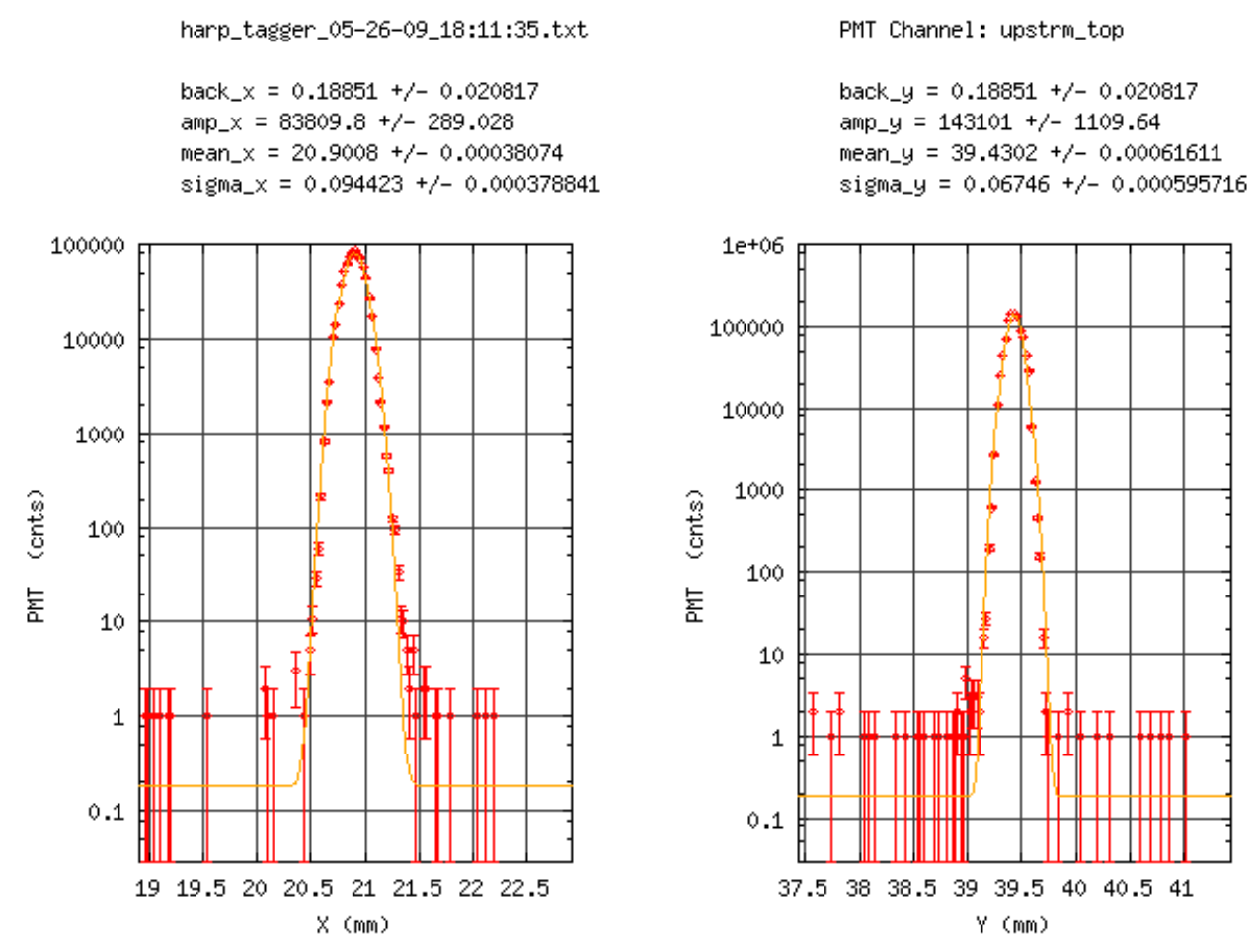

Fig. 2.7: Electron beam position $(\mathrm{x}, \mathrm{y})$ from a harp scan during the EG1-DVCS experiment. 


\subsubsection{Electron Beam Raster System}

When running on polarized targets, the electron beam is circularly rastered by a set of raster magnets upstream of the CLAS target to reduce target heating which would otherwise lower the target polarization. The current going to the raster magnets is recorded by ADCs in coincidence with each scattering event, so that the raster pattern can be reconstructed and monitored. Offline, the ADC information is translated into $\mathrm{x}$ and $\mathrm{y}$ position information relative to the beam line and used for correcting particle vertex reconstruction (Section 4.3). Figure 2.8 shows an ADC readout for the raster $\mathrm{x}$ and $\mathrm{y}$ positions during an EG1-DVCS run.
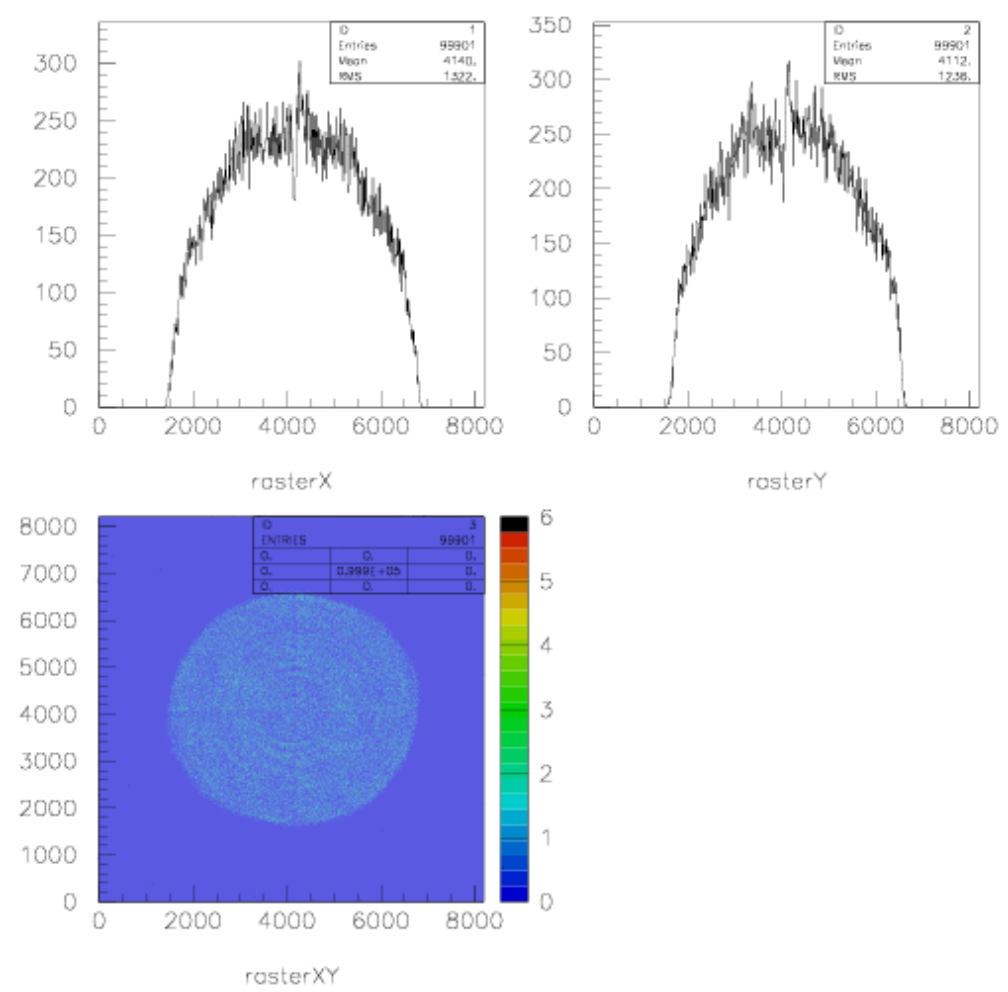

Fig. 2.8: Electron beam potion $(\mathrm{x}, \mathrm{y})$ after rastering during the EG1-DVCS experiment. 


\subsubsection{Electron Beam Current Monitor}

CLAS's Faraday cup is composed of $4000 \mathrm{~kg}$ of lead, which corresponds to 75 radiation lengths, and is located at the end of the beam line, $29 \mathrm{~m}$ downstream of CLAS center. Collected charge is measured through an electrical feedthrough. In addition to measuring the integrated charge, the Faraday cup also measures the variation of the charge with helicity for experiments requiring polarized electrons [28]. This information is written into the data stream. Knowledge of the accumulated charge allows for normalization of event counts which is essential for asymmetry measurements. Figure 2.9 shows charge counts from the Faraday cup plotted vs event number for one run of the EG1-DVCS experiment.

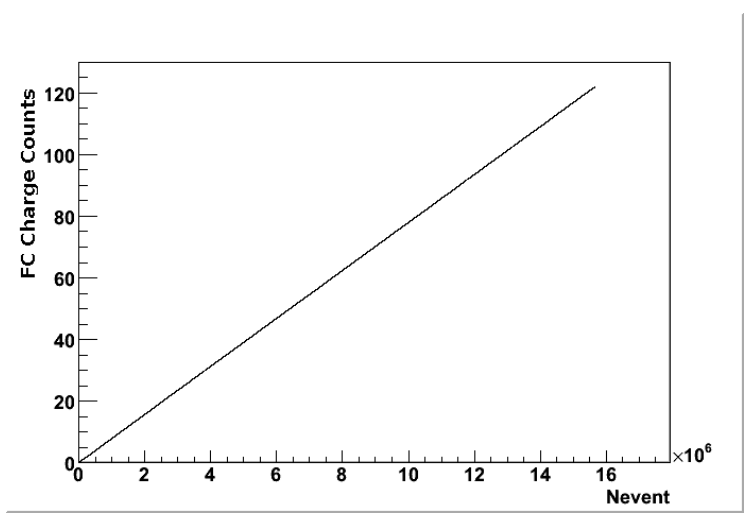

Fig. 2.9: Electron beam charge counts from the Faraday cup plotted vs event number for one run of the EG1-DVCS experiment. 


\subsection{Hall B CEBAF Large Acceptance Spectrometer}

The following section includes an overview of the separate detector systems that make up the Hall-B CEBAF Large Acceptance Spectrometer (CLAS), depicted in Figure 2.10. More extensive and detailed descriptions can be found in References [18]- [23]. Table 2.4 contains a summary of the overall coverage, resolution, and other characteristics of CLAS.

\begin{tabular}{|l|l|l|}
\hline Capability & Quantity & Range \\
\hline \hline Coverage & Charged-particle angle*,** & $8^{\circ} \leq \theta \leq 140^{\circ}$ \\
& Charged-particle momentum & $p \geq 0.2 \mathrm{GeV} / \mathrm{c}$ \\
& Photon angle* & $8^{\circ} \leq \theta \leq 45^{\circ}$ \\
& Photon energy* & $E_{\gamma} \geq 0.1 \mathrm{GeV}$ \\
\hline Resolution & Momentum $\left(\right.$ theta $\left.\lesssim 30^{\circ}\right)$ & $\sigma_{p} / p \approx 0.5 \%$ \\
& Momentum $\left(\right.$ theta $\left.\gtrsim 30^{\circ}\right)$ & $\sigma_{p} / p \approx(1-2) \%$ \\
& Polar angle & $\sigma_{\theta} \approx 1 \mathrm{mrad}$ \\
& Azimuthal angle & $\sigma_{\phi} \approx 4 \mathrm{mrad}$ \\
& Time (charged particles $)$ & $\sigma_{t} \approx(100-250) \mathrm{ps}^{\circ}$ \\
& Photon Energy* & $\sigma_{E} / \mathrm{E} \approx 10 \% / \sqrt{E}$ \\
\hline Particle ID & $\pi / p$ separation & $p \leq 3.5 \mathrm{GeV} / \mathrm{c}$ \\
& $\pi^{-}$misidentified as $e^{-}$ & $\leq 10^{-3}$ \\
\hline Luminosity & Electron beam & $L \approx 10^{34} \mathrm{nucleon} \mathrm{cm}^{-2} \mathrm{~s}^{-1}$ \\
\hline Data acquisition & Event rate & $4 \mathrm{kHz}$ \\
& Data rate & $25 \mathrm{MB} / \mathrm{s}$ \\
\hline
\end{tabular}

Table 2.4: Summary of the CLAS detector characteristics relevant to the EG1-DVCS experiment $[18] .{ }^{*}$ Coverage and resolution are listed without consideration of the additional Inner Calorimeter used in the EG1-DVCS experiment, see Table 2.5 for Inner Calorimeter characteristics. ${ }^{* *}$ The polarized target magnet (Section 2.6.3.1) reduces CLAS's maximum scattered particle theta angle to 50 


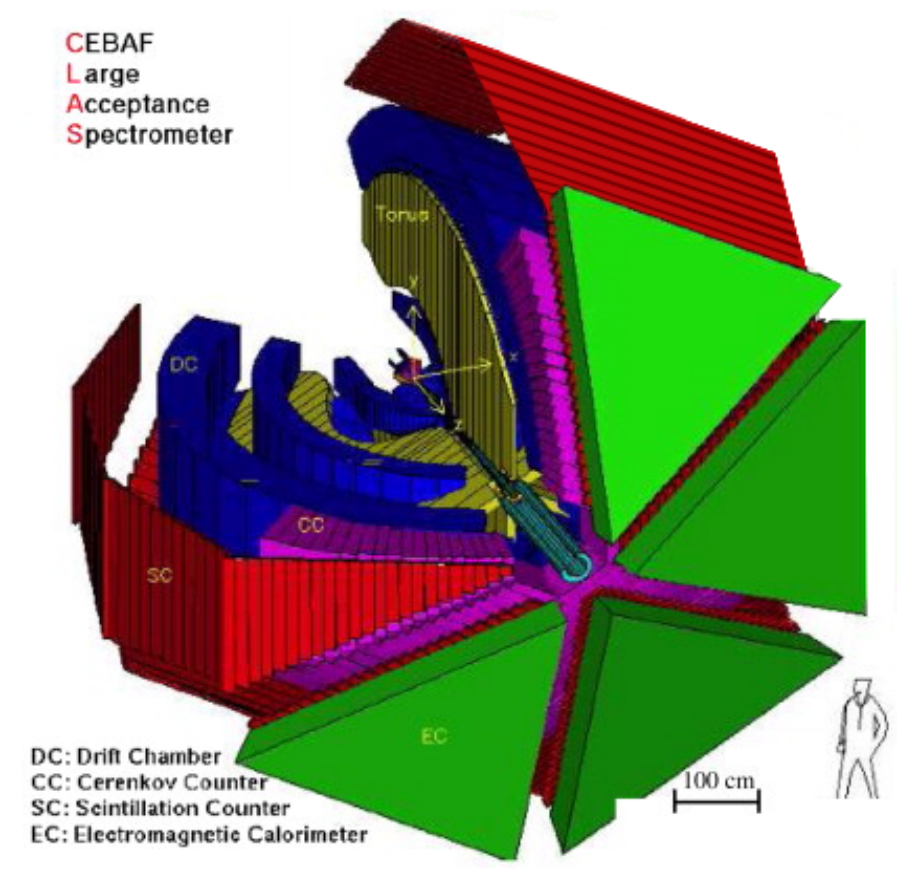

Fig. 2.10: The CEBAF Large Acceptance Spectrometer (CLAS) detector. Not pictured are the Large Angle Calorimeters which are not used in the EG1-DVCS experiment. 


\subsubsection{Torus Magnet}

The magnetic field for the momentum analysis of charged particles is generated by six superconducting coils arranged in a toroidal geometry around the electron beam line, as shown in Figure 2.11. The magnet is approximately $5 \mathrm{~m}$ in diameter and $5 \mathrm{~m}$ in length. The CLAS toroidal magnet bends charged particles toward or away from the beam axis but leaves the azimuthal angle essentially unchanged. The kidney-shape of the coils preserves a central field-free volume for the operation of a polarized target. Each of the six coils, which separate CLAS into 6 sectors, has four layers of 54 turns of aluminum stabilized $\mathrm{NbTi} / \mathrm{Cu}$ conductor cooled to a temperature of $4.5 \mathrm{~K}$.
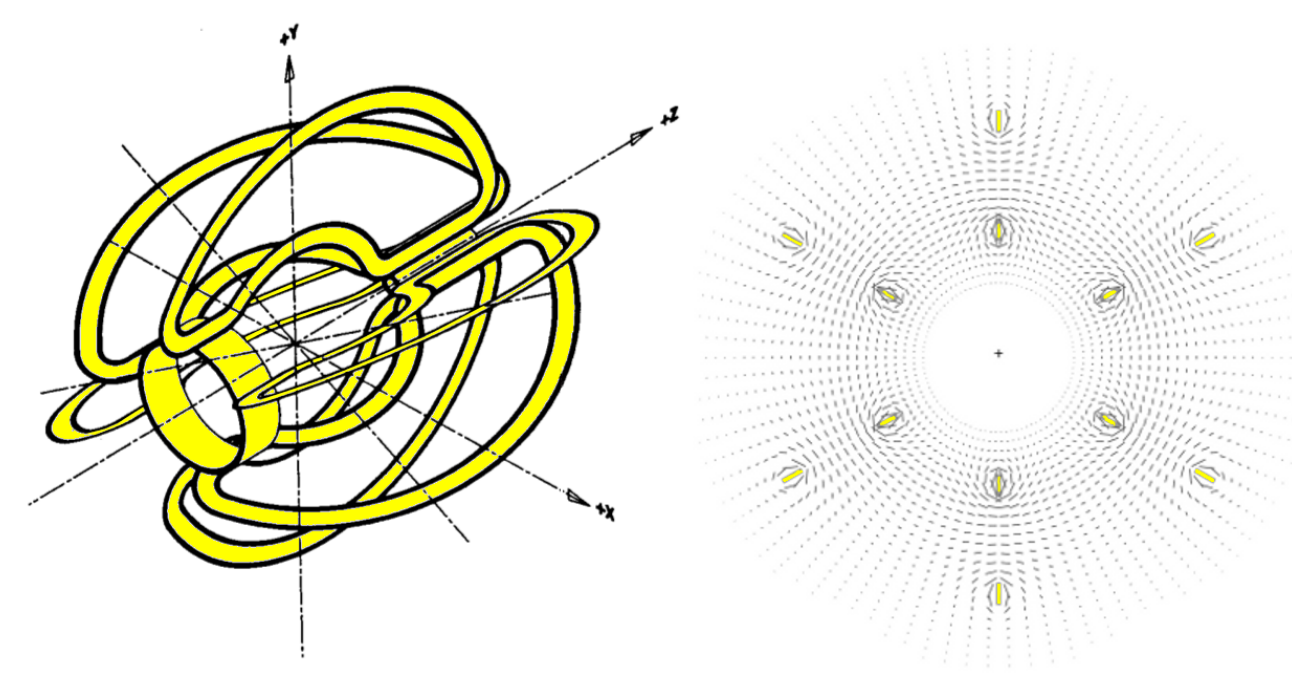

Fig. 2.11: The CLAS Torus configuration [19] (left) and magnetic field vectors for the CLAS toroid transverse to the beam in a plane centered on the target (right). The length of each line segment is proportional to the field strength at that point. [18].

\subsubsection{Drift Chambers}

Eighteen separate drift chambers are located at three radial positions in each of the six sectors of CLAS. These radial locations are referred to as Regions. The six Region One chambers surround the target in an area of low magnetic field, the six Region Two chambers 
are situated between the magnet coils in an area of high field near the point of maximum track sagitta, while the six Region Three chambers are located outside of the magnet coils. A schematic view of the regions can be seen in Figure 2.12, and the placement within the torus in Figure 2.13. Each chamber is subdivided into two superlayers of six wire layers each, one axial to the magnetic field, and the other tilted at a $6^{\circ}$ stereo angle to provide azimuthal information. The design of the wire layout is in a quasi-hexagonal pattern with six field wires surrounding one sense wire. A detail of the wire layout is shown in Figure 2.14.

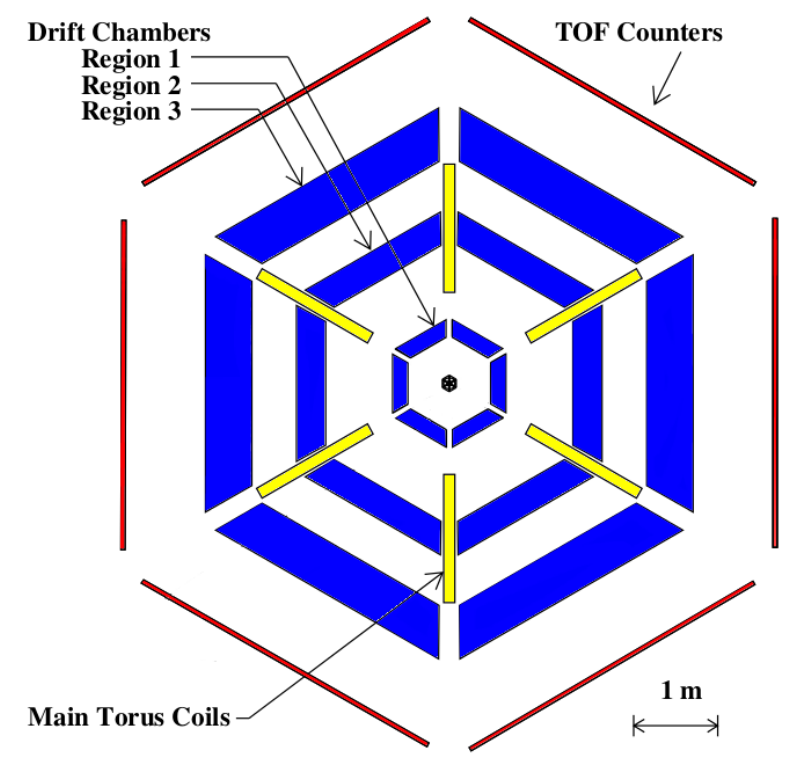

Fig. 2.12: Schematic view of CLAS showing a cut perpendicular to beam, with Torus (yellow), Drift Chambers (blue), and Time-of-Flight Counters (Red) [18]. 


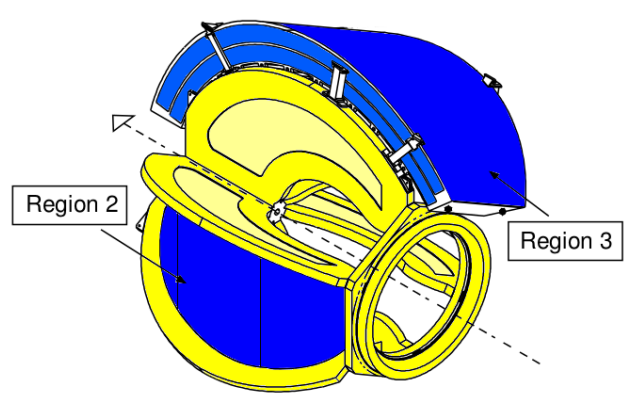

Fig. 2.13: The CLAS Torus cryostat (yellow) and a Region 2 (blue, lower left) and Region 3 (blue, upper right) Drift Chamber section [18].

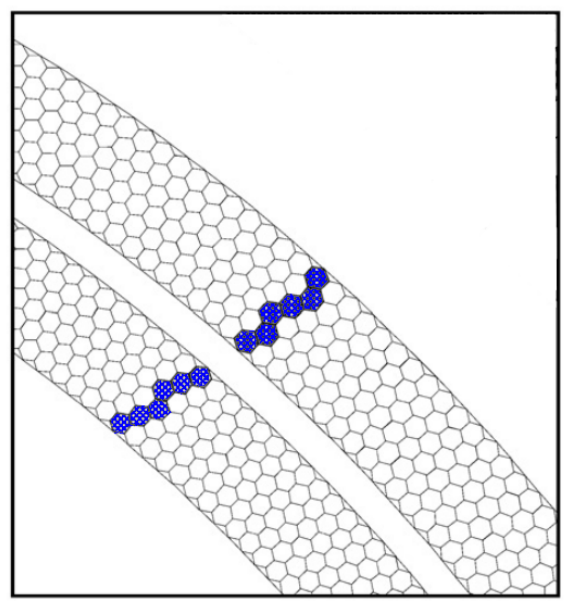

Fig. 2.14: A portion of the CLAS Region 3 Drift Chamber showing the layout of its two superlayers. A passing charged particle is shown by the blue highlighted drift cells that have fired [18]. 


\subsubsection{Cherenkov Counters}

The full $\theta$ range of each of CLAS's six sectors is divided into 18 regions, as may be seen in magenta in Figure 2.15. Each $\theta$ segment is divided into two modules about the symmetry plane bisecting each sector, see Figure 2.16. This results in a total of 12 identical (except for an inversion symmetry) subsectors around the $\phi$ direction for each $\theta$ interval, and a total of 216 light-collection modules. To maximize the available coverage in each of CLAS's 6 sectors the Cherenkov Counter's light-collecting cones and photomultiplier tubes (PMTs) were placed in the regions of $\phi$ that are already obscured by the Torus Magnet coils, and covering as much of the available unobstructed space as possible with mirrors. A drawing of the design can be seen in Figure 2.17.

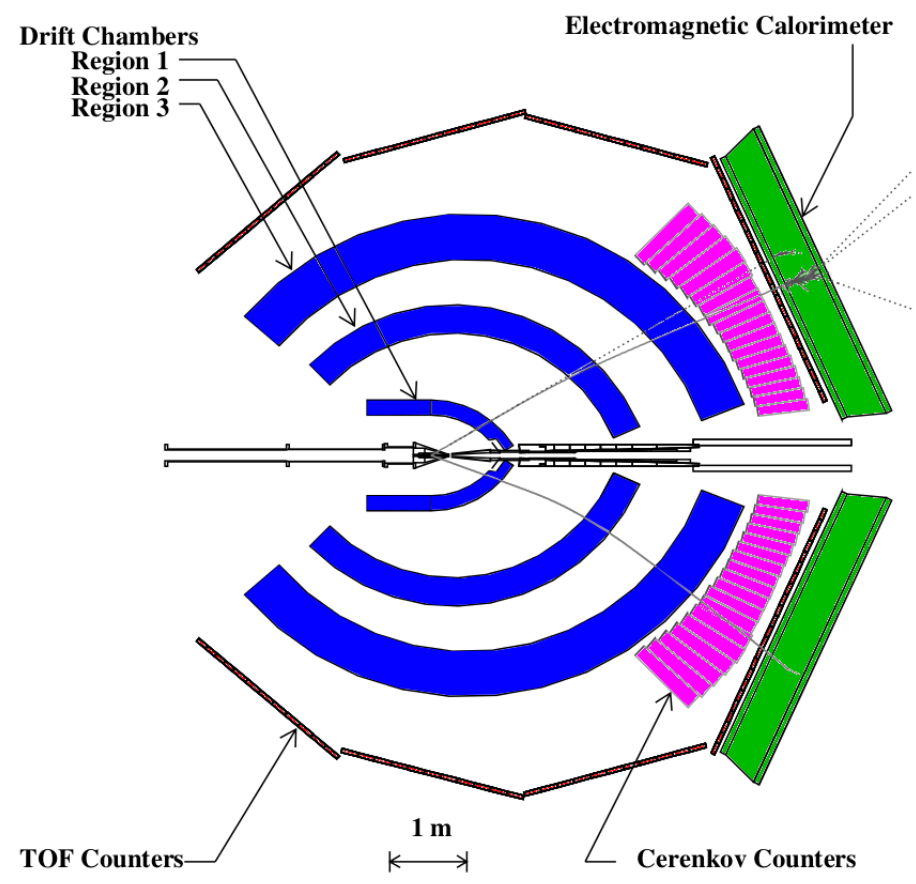

Fig. 2.15: Schematic view of CLAS showing a cut along the beam line with Drift Chambers (blue), Time-of-Flight Counters (red), Cherenkov Counters (magenta), and Electromagnetic Calorimeters (green) [21]. 


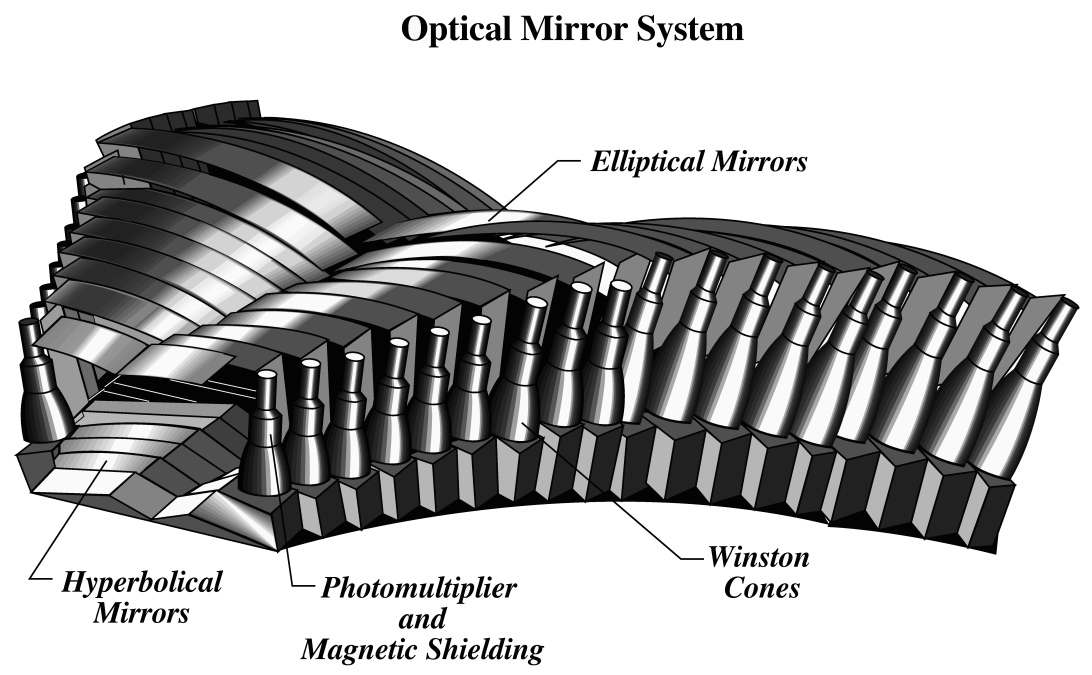

Fig. 2.16: A schematic drawing of the array of the Cherenkov optical modules in one sector of CLAS [21].

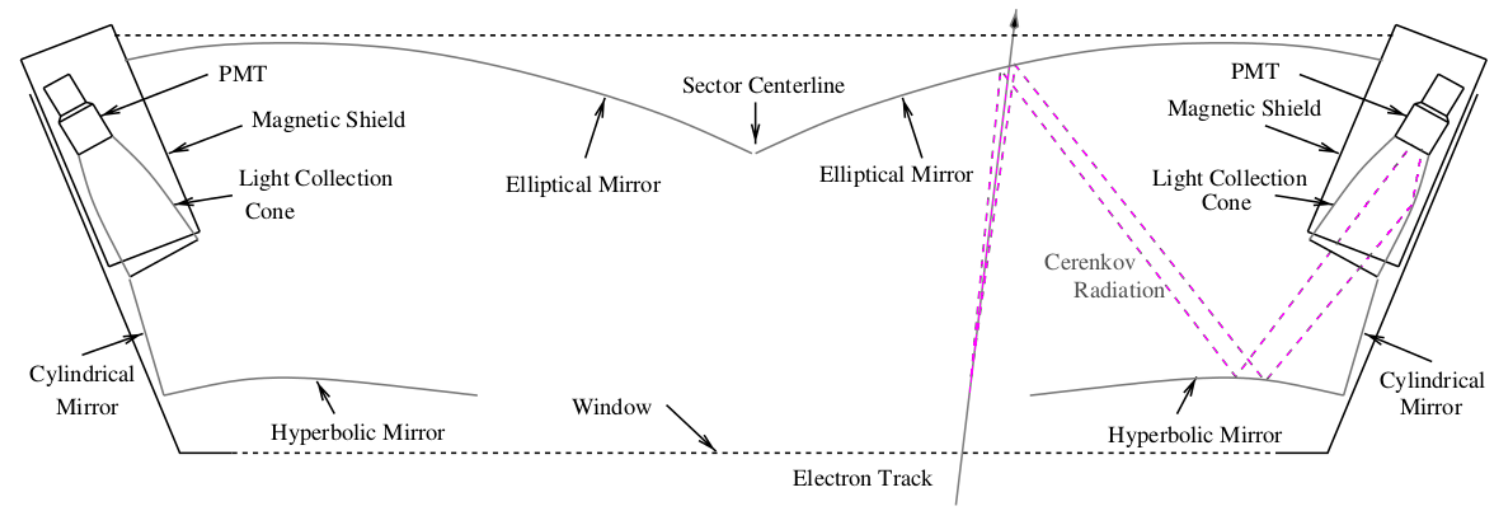

Fig. 2.17: Schematic diagram of one Cherenkov segment, symmetric about the sector center. Also shown, in magenta, is an example of an electron trajectory with the collection of Cherenkov light to the PMT [18]. 


\subsubsection{Time-of-Flight Counters}

The Time-of-Flight Counters' design is based on long rectangular plastic scintillators, each with two PMTs, one on each end. The scintillators are positioned outside the tracking system between the Cherenkov Counters and the Electromagnetic Calorimeters, as can be seen in red in Figure 2.15. Each of CLAS's six sectors has 57 scintillators in 4 groups called panels, as in Figure 2.18. Panel 1 contains 23 scintillators which are referred to as the forward-angle counters, and correspond to scattering angles less than $46^{\circ}$. Panels 2, 3 and 4 , which contain 11, 11 and 12 scintillators respectively, are called large angle counters covering roughly $47^{\circ}$ to $141^{\circ}$ scattering angles.

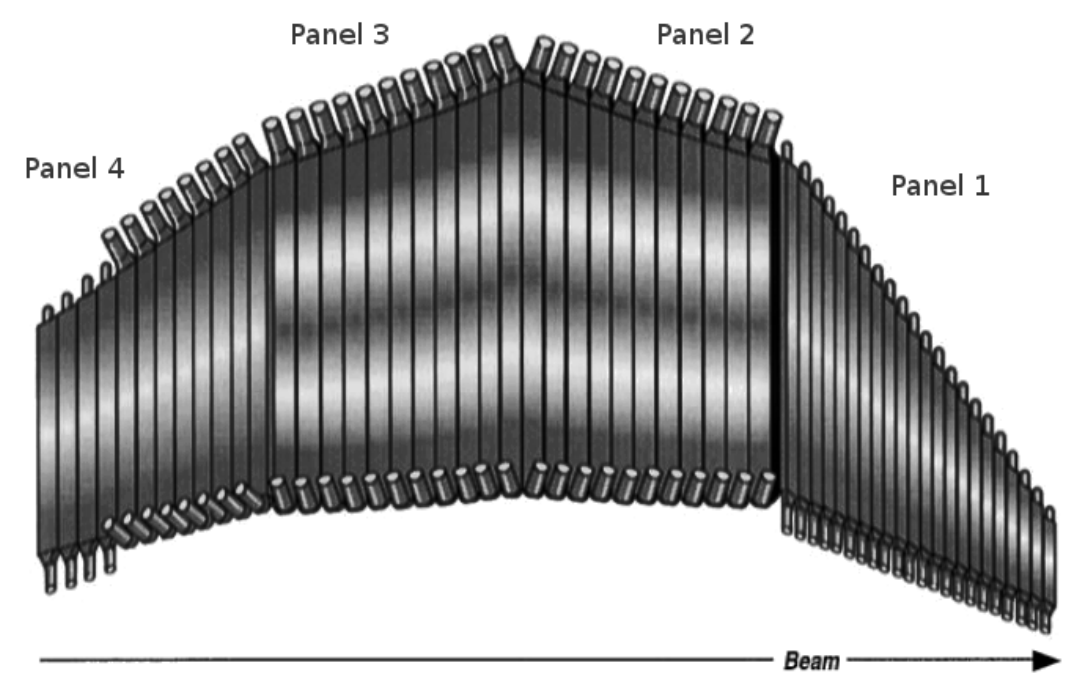

Fig. 2.18: A schematic drawing of the array of Time-of-Flight scintillators in one sector of CLAS [21]. 


\subsubsection{Electromagnetic Calorimeter}

The Electromagnetic Calorimeters are positioned on the outermost portion of CLAS, as can be seen in green in Figure 2.15. There are 6 calorimeters covering the six sectors of CLAS, covering a $\theta$ range from $8^{\circ}$ to $45^{\circ}$. Each calorimeter consists of 39 layers of scintillators separated by layers of lead. Each scintillator layer consists of 36 strips parallel to one side of the triangle, with the orientation of the strips rotated by $120^{\circ}$ in successive layers, illustrated in Figure 2.19. This makes for three orientations, or views (labeled U, V, and W), each containing 13 layers. Each view is further subdivided into an inner (5 layers) and outer (8 layers) stack.

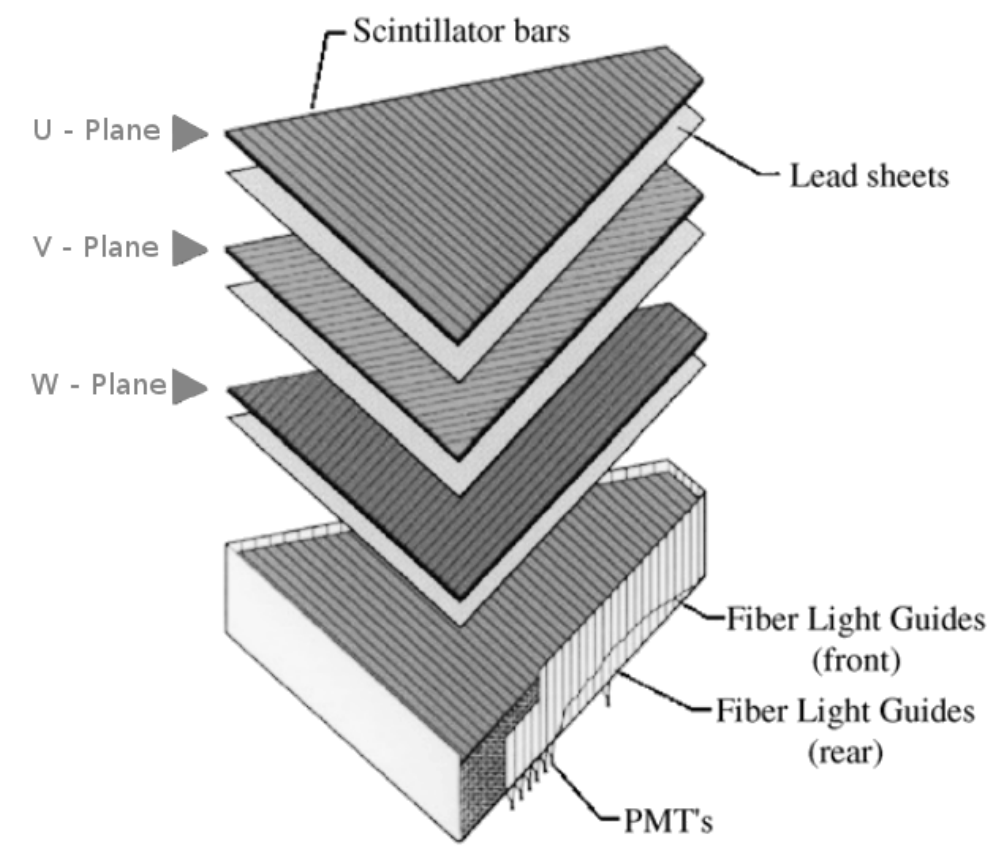

Fig. 2.19: Flared view of one of the six CLAS electromagnetic calorimeter modules. [18]. 


\subsubsection{Inner Calorimeter}

For the EG1-DVCS experiment, the CLAS detector was equipped with an Inner Calorimeter located between the polarized target assembly and the Drift Chambers Region 1, see Figure 2.21. The Inner Calorimeter consists of 424 lead-tungsten $\left(\mathrm{PbWO}_{4}\right)$ crystals, each crystal outfitted with an avalanche photo-diode for light readout. The crystals are arranged in an octagonal array, as shown in Figure 2.20. The photon angular coverage of the Inner Calorimeter overlaps with CLAS, reducing CLAS's low angle coverage for both charged and neutral particles. For Run Period A where the target was $57.95 \mathrm{~cm}$ from the IC (located at CLAS center) the photon coverage in $\theta$ was from $4^{\circ}$ to $15.5^{\circ}$, while the housing of the Inner Calorimeter blocked the $\theta$ photon coverage from roughly $15.5^{\circ}$ to $17^{\circ}$. For charged particles, the IC blocked CLAS's $\theta$ coverage up to $17^{\circ}$. For Run Period B where the target was 67.97 $\mathrm{cm}$ from the IC (again located at CLAS center) the photon coverage in $\theta$ was from $3.5^{\circ}$ to $13^{\circ}$, while the housing of the Inner Calorimeter blocks the $\theta$ region from roughly $13^{\circ}$ to $15^{\circ}$. For charged particles in Run Period B, the IC blocked CLAS's $\theta$ coverage up to $15^{\circ}$.A summary of the IC coverage and resolution is listed in Table 2.5.

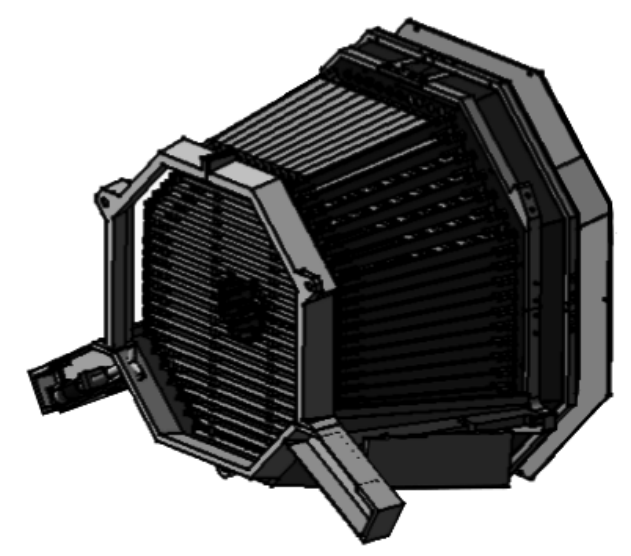

Fig. 2.20: A schematic drawing of the Inner Calorimeter. 


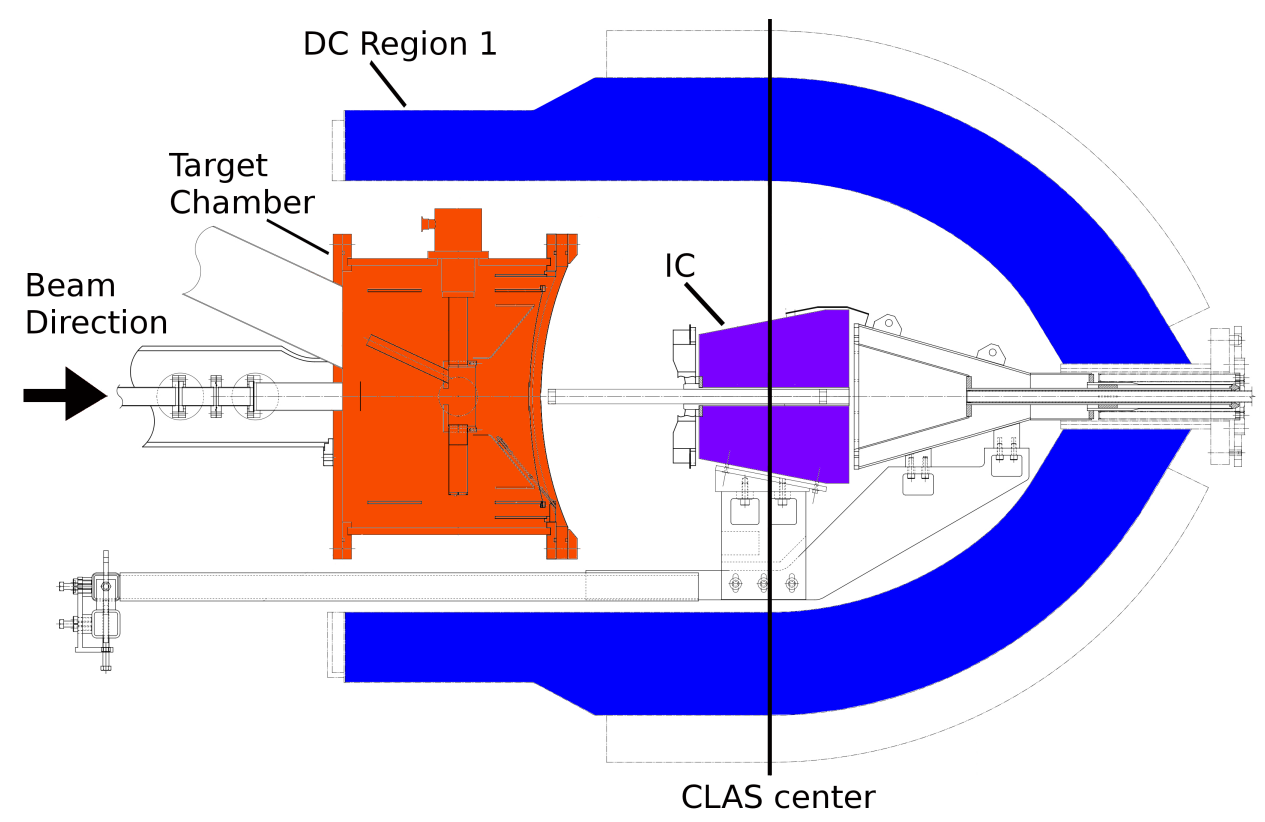

Fig. 2.21: A schematic drawing of the Inner Calorimeter (purple), Drift Chamber Region 1 (blue), and Polarized Target system (orange).

\begin{tabular}{|l|l|l|}
\multicolumn{1}{c}{ Capability } & \multicolumn{2}{c}{ Range } \\
\hline \hline Shadow on CLAS & Charged particle angle Run Period A & $\theta \leq 17^{\circ}$ \\
& Charged particle angle Run Period B & $\theta \leq 15^{\circ}$ \\
\hline Coverage & Photon angle Run Period A & $4^{\circ} \leq \theta \leq 15.5^{\circ}$ \\
& Photon angle Run Period B & $3.5^{\circ} \leq \theta \leq 13^{\circ}$ \\
& Photon energy & $E_{\gamma} \geq 0.015 \mathrm{GeV}$ \\
\hline Resolution & Photon Energy (at 1 GeV) & $\sigma_{E} / E \approx 4 \%$ \\
\hline
\end{tabular}

Table 2.5: Summary of the IC detector characteristics. 


\subsection{Data Processing}

During the experiment, the signals coming from each CLAS detector component for events which pass CLAS's two-level trigger system are stored in BOS bank files. The data is then processed (off-line) converting the the raw information of the ADCs and TDCs into physical observables like momentum, trajectory, etc... The processing also applies detector calibrations, which are determined through well established CLAS calibration programs for each detector system. Due to the large size of the data files, a general particle skim was used by the EG1-DVCS working group which implemented some very loose particle identification cuts as well as using bit compression in order to reduce the size of the data files by a factor of 10. The details of the event selection cuts and the structure of the data files are detailed in Reference [29].

\subsection{Polarized Target}

The source of polarized protons used in the EG1-DVCS experiment was dynamically polarized solid state ${ }^{14} \mathrm{NH}_{3}$ (Figure 2.22). The ${ }^{14} \mathrm{NH}_{3}$ was polarized using the process of Dynamic Nuclear Polarization (DNP), discussed below.

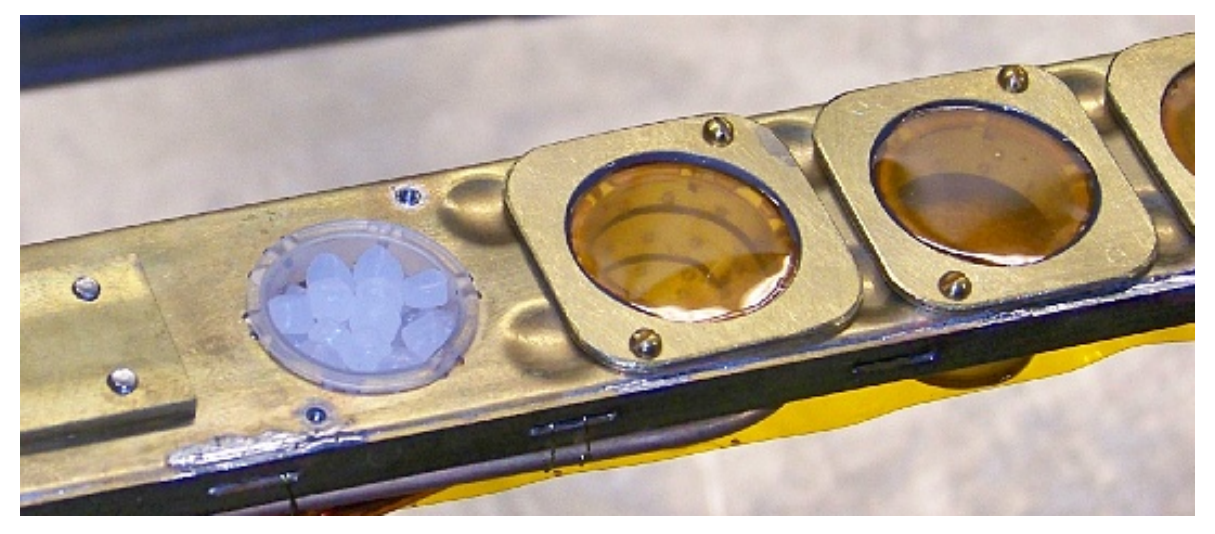

Fig. 2.22: Photograph of the section of the target insert housing the target cups. Cup 1 (left) contains beads of solid state ${ }^{14} \mathrm{NH}_{3}$. The right 3 cups show the Kapton covers used to encapsulate the target material. 


\subsubsection{Dynamic Nuclear Polarization}

There are two ways in which to describe the process of Dynamic Nuclear Polarization (DNP). The first is through the solid state effect, the second is through equal spin temperature theory. The target material for the EG1-DVCS experiment, ${ }^{14} \mathrm{NH}_{3}$, does not follow either description exactly, but includes aspects of both descriptions. It is important to note that both descriptions result in the need of the same apparatus as well as accurately describe the observed results. Both descriptions start with a polarizable material defined as a material with unpaired protons with embedded paramagnetic centers. In the case of the EG1DVCS target material, the unpaired protons come from the 3 unpaired protons in the ${ }^{14} \mathrm{NH}_{3}$ molecule, and the paramagnetic centers from irradiating the target material. The process starts with placing the target in a large magnetic field $(\sim 5 T)$ at low temperature $(\sim 1 K)$, effectively polarizing the electrons (99\%) in the sample, and to a much lesser extent the protons $(0.5 \%)$. Called thermal equilibrium polarization, the value for spin $1 / 2$ particles is given by the Brillouin function, Equation 2.2 where $\mu$ is the magnetic moment of the proton, $k_{N}$ is Boltzmann's constant, $B_{0}$ is magnetic field, and $T$ is temperature.. Overviews of the two polarization enhancing processes are given in the following sections.

$$
P_{T E}=P_{1 / 2}=\tanh \left(\frac{\hbar \omega}{2 k_{B} T}\right)=\tanh \left(\frac{\mu B_{0}}{k_{B} T}\right)
$$

\subsubsection{Polarization Through a Solid State Approach}

The solid state approach is the simplest picture of the DNP process. Electron paramagnetic resonance (EPR) frequency microwave radiation can be used to induce electron spin flips from a spin orientation of anti-parallel to parallel with the magnetic field. If the microwave frequency is instead selected higher or lower than the electron paramagnetic resonance frequency by an amount equaling the nuclear magnetic resonance (NMR) frequency of the proton, then instead of an electron spin flip one induces a double electron-proton spin flip. As an example, if one uses a microwave frequency equal to $\nu=\nu_{E P R}-\nu_{N M R}$ one induces the transition $e_{\downarrow} p_{\Downarrow} \rightarrow e_{\uparrow} p_{\Uparrow}$. The energy levels and transitions of this system are shown in Figure 2.23. The relatively fast relaxation time of the electron soon produces the transition $e_{\uparrow} p_{\Uparrow} \rightarrow e_{\downarrow} p_{\Uparrow}$. Exploiting the fast electron relaxation effectively pumps the protons in a 
chosen polarization state, in the above example to the $p_{\Uparrow}$ state. The same operation can be used to produce the opposite proton polarization state without changing the magnetic field by inducing the $e_{\downarrow} p_{\Uparrow} \rightarrow e_{\uparrow} p_{\Downarrow}$ through supplying the system with $\nu=\nu_{E P R}+\nu_{N M R}$. The

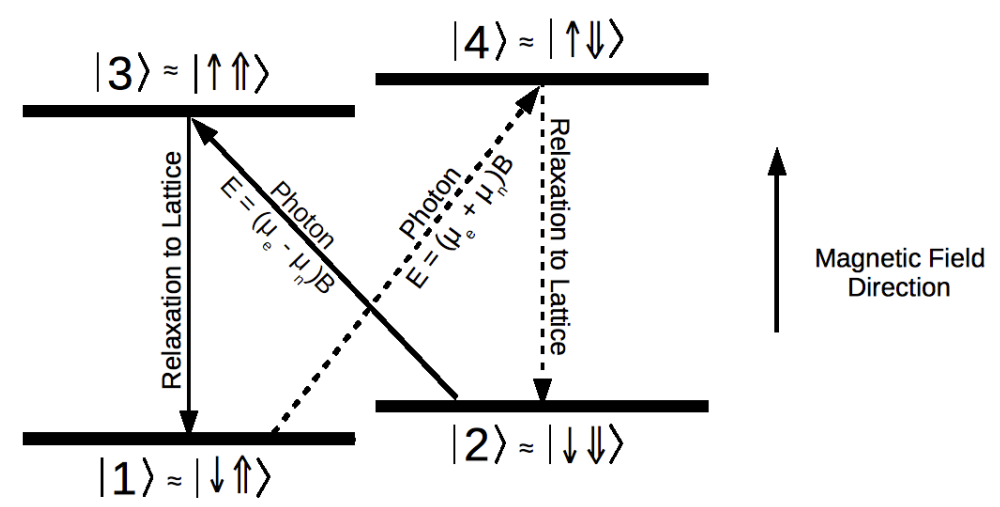

Fig. 2.23: Transitions involved in polarization enhancement by DNP. $\uparrow$ and $\Uparrow$ denote electron and proton spin states respectively.

rate of proton polarization is given in Equation 2.3 with $N_{S / I}$ the number of paramagnetic centers and of protons respectively, $P_{S / I}$ the electron/proton polarization, $T_{S / I}$ the relaxation time of the electron/proton, and $V$ the probability per unit time of a proton flipping with the electron in the presence of the microwave radiation. The superscript $L$ denotes thermal equilibrium, i.e. no microwave radiation.

$$
\begin{aligned}
& \frac{P_{S}}{d t}=-V\left(P_{S}-P_{I}\right)+\frac{1}{T_{S}}\left(P_{S}^{L}-P_{S}\right) \\
& \frac{P_{I}}{d t}=\frac{N_{S}}{N_{I}} V\left(P_{S}-P_{I}\right)-\frac{1}{T_{I}}\left(P_{I}-P_{I}^{L}\right)
\end{aligned}
$$

An estimate of the maximum achievable polarization can be determined by setting the derivatives in Equation 2.3 to 0 and assuming the total rate of relaxation of the electrons is much greater than the protons', i.e. $N_{1} / T_{1} \ll N_{S} / T_{S}$ (a valid assumption for the EG1-DVCS target material). The resulting maximum polarizations work out to Equation 2.4. If one further assumes the rate of protons being flipped with electrons is much greater than the total relaxation rate of the protons, $N_{S} V \gg N_{I} / T_{I}$, then the proton polarization can reach that of the electron thermal equilibrium polarization, $P_{S} \approx P_{I}$.

$$
\begin{aligned}
P_{S} & \approx P_{S}^{L} \\
P_{I} & \approx \frac{P_{I}^{L} \frac{N_{I}}{N_{S} T_{I} V}+P_{S}^{L}}{\frac{N_{I}}{N_{S} T_{I} V}+1}
\end{aligned}
$$




\subsubsection{Polarization Through Equal Spin Temperature Theory}

The Equal Spin Temperature Theory has the advantage of including in it the interaction of the free electrons, which is particularly important in target materials whose paramagnetic centers concentration is high. The inclusion of the electron-electron interactions results in a system that no longer has discrete energy states. The electron system can be described by Boltzmann distributions with temperatures $T_{Z}$ (electron Zeeman interaction) and $T_{S S}$ (spin-spin interaction). The Zeeman interaction associated with $T_{Z}$ determines the overall population of the two continuous spin states, whereas the spin-spin interaction, $T_{S S}$, determines the Boltzmann distribution within each state. Thermal equilibrium of the electron system occurs when $T_{S S}=T_{Z}$. The protons in the material can be described by Boltzmann distributions characterized by temperature $T_{Z_{p}}$, associated with proton Zeeman system. The coupling of this system with the electron spin-spin system allows the proton system to cool by a double electron spin flip along with a single proton spin flip, enabling proton polarization enhancement.

The polarization enhancement process involves microwave radiation slightly off from the electron's Larmor frequency (the Larmor frequency being equal to the EPR frequency in the limit of free electrons) to increase or decrease $T_{S S}$. Microwave radiation equaling $h(\nu+\delta)$ is absorbed by the electron. $h \nu$ is the electron Zeeman energy and is absorbed by the electron Zeeman system, and $h \delta$ is absorbed by the spin-spin system. If $\delta<0$ the electron spin-spin system emits this energy, if $\delta>0$ the electron spin-spin system absorbs the energy. For a positive $T_{S S}$ the act of emitting effectively cools the system, and absorbing heats the system. Conversely, for a negative $T_{S S}$, which corresponds to a negative polarization, the act of absorbing cools the system, and emitting heats the system. The proton Zeeman system, $T_{Z_{p}}$, moves to thermal equilibrium with the thermal equilibrium of the electron spin-spin system, $T_{Z_{p}}=T_{S S}$, by double electron spin flips coupled with a single proton spin flip. In this process, the spin of the electron system effectively stays the same, where as the proton system emits or absorbs $h \nu_{p}$ with $\nu_{p}$ being the Larmor frequency of the proton. As $T_{Z_{p}}$ decreases, the proton polarization increases. If the temperature of the spin-spin system, $T_{S S}$, is negative, the thermal contact between the spin-spin system and the proton Zeeman 
system, $T_{Z_{p}}$, would result in a negative value for $T_{Z_{p}}$, which corresponds to a negative proton polarization.

\subsubsection{Target Material}

There are a multitude of materials which can be dynamically polarized, each material having advantages and disadvantages. Typically used materials that can reach $90 \%$ proton polarization or more include butanol $\left(\mathrm{C}_{4} \mathrm{H}_{9} \mathrm{OH}\right)$, lithium hydride $(\mathrm{LiH})$, and ammonia $\left(\mathrm{NH}_{3}\right)$ [30]. $\mathrm{NH}_{3}$ was chosen because of the material's high resistance and easy repairability to radiation damage.

The material was prepared by first freezing the $\mathrm{NH}_{3}$ gas using liquid nitrogen. Then, to increase surface area to make cooling easier, the $\mathrm{NH}_{3}$ solid was crushed it into small pieces, approximately $1-3 \mathrm{~mm}$ in diameter (Figure 2.22). Paramagnetic centers in the form of free electrons are then introduced through irradiation with an electron beam. Electrons from the beam knock out a proton from the $\mathrm{NH}_{3}$ forming $\mathrm{NH}_{2}^{-}$where the extra electron acts as a paramagnetic center for the DNP process.

As the electroproduction experiment EG1-DVCS was running, the experiment's electron beam further ionized the target material. Eventually the build up of paramagnetic centers causes a negative effect on the proton polarization, primarily through broadening the EPR transition and increasing the number of relaxation paths for the protons [24]. These excess centers can be removed from the material by a process of annealing, where the temperature of the target is increased from the operational temperature of $1 \mathrm{~K}$ to around $80 \mathrm{~K}$ for a period of time, usually around 45 minutes. Heating the system results in some of the created ions to combine, i.e. $\mathrm{NH}_{2}^{-}+\mathrm{H}^{+} \rightarrow \mathrm{NH}_{3}$. This process was done about once a week, or if the average achievable proton polarization fell by more than $5 \%$.

\subsubsection{Target Design}

The subsystems of the polarized target include a superconducting magnet, a $1 \mathrm{~K}$ refrigerator, microwave and NMR systems, and a target insert. A review of each subsystem is described separately in the following sections. Figure 2.21 shows how the magnet and target chamber 
containing the target insert (in orange) of the polarized target assembly is situated with respect to the CLAS detector (discussed in section 2.4).

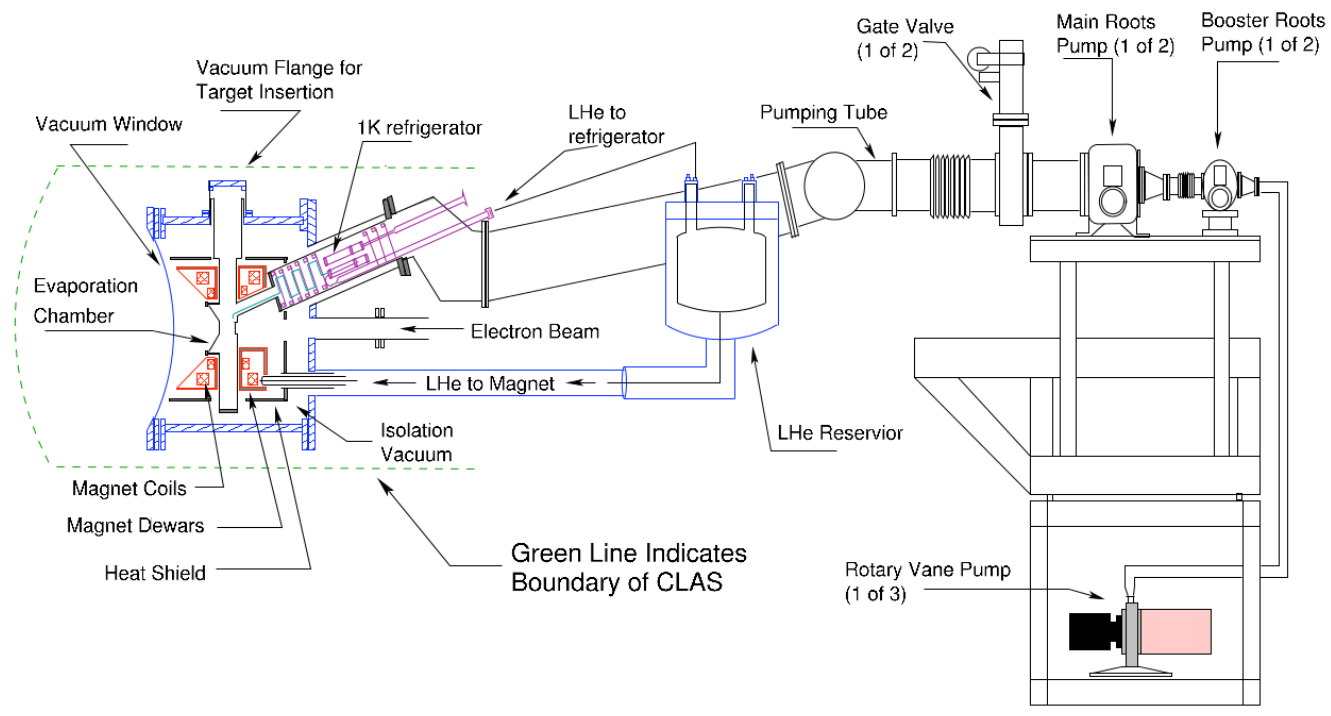

Fig. 2.24: Cutaway view of the polarized target cryostat from the beam-left side [31]. 


\subsubsection{Superconducting Magnet}

The superconducting magnet surrounds the target chamber and consists of a pair of Helmholtz coils constructed out of a Niobium-Titanium alloy submerged in liquid ${ }^{4} \mathrm{He}$ supplied by a reservoir located outside of the CLAS detector. The magnet housing and liquid ${ }^{4} \mathrm{He}$ reservoir are indicated in blue in Figure 2.24.

Niobium-Titanium alloy has a critical temperature of $\sim 9.2 K$. Kept below this temperature, the magnet becomes superconducting and can be maintained in a persistent current mode. In persistent mode, the leads connecting the magnet to the power supply are shorted, isolating the magnet from the power supply and any associated power fluctuations, increasing field stability. Persistent mode also reduces the liquid helium consumption because the power supply can be turned off thereby adding less heat into the system. During the EG1-DVCS experiment runs the magnet was kept in persistent mode.

The magnet produces a $5 T$ magnetic field parallel to the electron beam axis. Within a $20 \mathrm{~mm}$ diameter $20 \mathrm{~mm}$ length cylindrical volume, the central field uniformity varies less than $10^{-4}$. This high uniformity is essential for the DNP and NMR processes [31]. The field falls off as $\sim 1 / r^{3}$ from this central region, becoming negligible at the Region 1 Drift Chambers. The magnetic field and its effect on charged particles is accounted for in the CLAS particle reconstruction programs. The on-axis bore of the magnet is $200 \mathrm{~mm}$ in diameter, which limits the forward (downstream) scattered particle maximum $\theta$ acceptance angle to $\sim 50^{\circ}$.

\subsubsection{Target Insert}

The EG1-DVCS experiment ran on four separate targets. Each target was housed in a cell consisting of a polychlorotrifluoroethylene (PCTFE) cylinder capped with kapton. The cells were mounted on a thin aluminum structure, shown in Figure 2.22. Two of the target cells, also referred to as "cups", contained polarized ${ }^{14} \mathrm{NH}_{3}$, one cup contained ${ }^{12} \mathrm{C}$, and one cup was left empty. The ${ }^{12} \mathrm{C}$ and empty targets were used for nuclear and other background studies. The two ${ }^{14} \mathrm{NH}_{3}$ cups were outfitted with NMR coils located on the outside of the PCTFE cylinders for polarization monitoring (Section 2.6.4). A schematic drawing of the 
target insert showing the placement of the target cells and NMR coils is shown in Figure 2.25. The aluminum structure that housed the target cups was designed to be moved vertically with a precision of $2 \mu \mathrm{m}$ remotely, thereby allowing a change of target without entering the experimental hall.

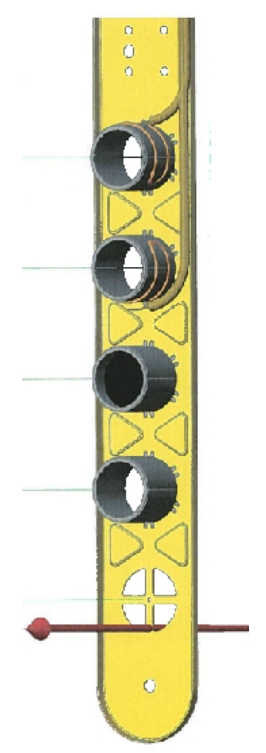

Fig. 2.25: Schematic drawing of the polarized target insert showing the four target cups. The two top cups include NMR coils for ${ }^{14} \mathrm{NH}_{3}$ polarization monitoring. Below the four target cups is a cross-hair for positional calibration of the target insert. 


\subsubsection{Refrigerator}

The targets were kept at an operational temperature of $1 K$ by being submerged in a pumped bath of liquid ${ }^{4} \mathrm{He}$. They were housed in an evaporation chamber situated at the center of the Helmholtz coils, consisting of a stainless steel chamber of $50 \mathrm{~cm}$ circumference and $7 \mathrm{~cm}$ length. The up-stream end of the chamber is connected to the ${ }^{4} \mathrm{He}$ refrigerator pumping tube. The refrigerator consists of a separator, heat exchangers, and two pumping tubes. Its position on the polarized target assembly can be seen in magenta in Figure 2.24. Liquid ${ }^{4} H e$, from the same reservoir that services the superconducting magnet, enters the separator, a copper pot, where the ${ }^{4} \mathrm{He}$ vapor is pumped away. The liquid from the separator drains into one of the two the $2.5 \mathrm{~mm}$ diameter copper pumping tubes. One of the tubes, called the 'bypass' line, is a direct line into the evaporation chamber and is used in the initial cool down of the system. The other, called the 'run' line, is heat sunk to a series of seven perforated copper plates that act as liquid-gas heat exchangers between the incoming liquid and the vapor pumped from the target chamber thereby further cooling the liquid before it enters the evaporation chamber.

\subsubsection{Microwave System}

The microwaves necessary to polarize the ${ }^{14} \mathrm{NH}_{3}$ target material were generated by an Extended Interaction Oscillator (EIO) capable of several watts of power at $140 \mathrm{GHZ}$ with a linewidth of $\sim 10 \mathrm{MHz}$ [31]. The central frequency can be tuned over a bandwidth of $2 \mathrm{GHz}$ by adjusting the length of the resonant cavity, which is done mechanically using a remotely controlled DC motor. This tunability allows for polarization in both the positive(parallel to the electron beam) and negative(antiparallel to the electron beam) directions without reversing the field of the target magnet.

The microwaves were transmitted from the EIO outside the target cryostat through rectangular wave guides and once inside the cryostat through a $5 \mathrm{~mm}$ copper-nickel $(\mathrm{CuNi})$ tube. The $C u N i$ tube terminated with a gold plated rectangular horn, which can be seen in Figure 2.26. 


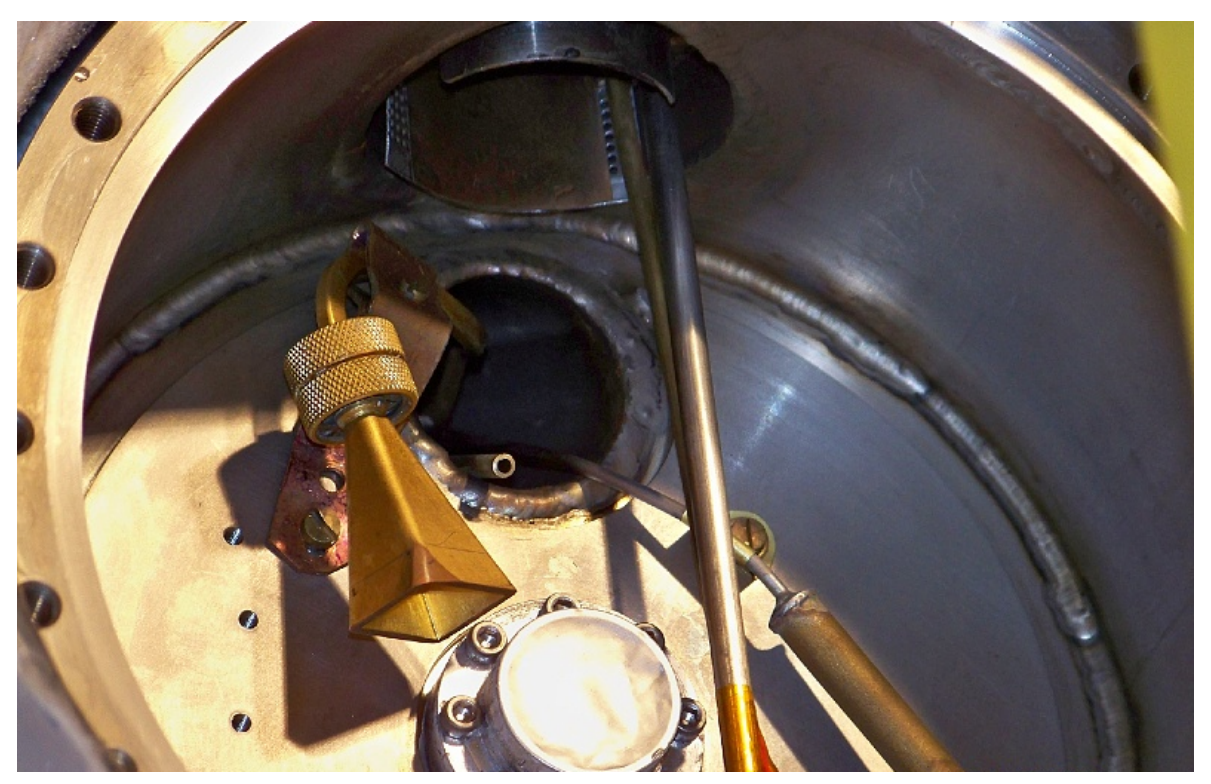

Fig. 2.26: Picture of the polarized target evaporation chamber and rectangular microwave horn looking upstream. 


\subsubsection{Nuclear Magnetic Resonance}

Nuclear Magnetic Resonance (NMR) was used to monitor the proton's polarization during the experiment. Measuring the NMR signal involves inducing and detecting nuclear magnetic transitions. The rate of these transitions is proportional to the population differences between the energy levels, i.e. the polarization. The premise of measuring the NMR signal of the polarized protons is that the impedance of an inductor is related to the magnetic susceptibility of the material inside the inductor.

The polarized target material is surrounded by an inductor coil which produces a magnetic field $\left(B_{1}\right)$ that is perpendicular to the target magnetic field $\left(B_{0}\right)$ with $B_{1}<<B_{0}$. Choosing the z-axis along the direction of $B_{0}$ and x-axis along $B_{1}$, the susceptibility of the target material in relation to $B_{1}$ can be decomposed into a real (dispersive) and imaginary (absorptive) part as expressed in Equation 2.5 where $M_{x^{\prime}}$ and $M_{y^{\prime}}$ are the target material's magnetization in the $x^{\prime}, y^{\prime}$ plane.

$$
\chi(\omega)=\chi^{\prime}(\omega)+i \chi^{\prime \prime}(\omega)=\frac{\mu_{0}}{2 B_{1}}\left(M_{x^{\prime}}(\omega)+i M_{y^{\prime}}(\omega)\right)
$$

The coil's inductance becomes Equation 2.6, with $\omega$ being the frequency going through the inductor, $L_{0}$ the inductance of the empty coil, and $\eta$ the fill factor of the material in relation to the coils.

$$
L(\omega)=L_{0}(1+\eta \chi(\omega))
$$

As the frequency through the inductor is swept through a range and near resonance, i.e. $\omega$ $=$ the proton Larmor frequency, the dispersive part of the magnetic susceptibility, $\chi^{\prime}$, will be at a minimum and the absorptive part, $\chi^{\prime \prime}$, a maximum. Measurement is taken of the change in inductance throughout the sweep through the resonance region using a Q-meter circuit (Section 2.6.4.1). The strength of the signal at resonance is proportional to the polarization in the $z$ direction with the absolute polarization of the material being proportional to the integral of $\chi^{\prime \prime}$ as Equation 2.7 [32]. There, $C$ is a constant of proportionality, also called calibration constant discussed in Section 2.6.4.1. It is important to note that because $B_{1}<<$ $B_{0}$ this measurement does not appreciably affect the target polarization in the $z$ direction.

$$
P_{z}=C \int_{\Delta \omega} \chi^{\prime \prime}(\omega) d \omega
$$




\subsubsection{Polarization Monitoring and Calibration}

Continuously throughout the experiment, Nuclear Magnetic Resonance (NMR) measurements of the $\mathrm{NH}_{3}$ target were taken. These measurements were calibrated using thermal equilibrium (TE) measurements, and the average polarization for each run was recorded. The polarization of protons at temperature $T$ in a magnetic field $B_{0}$ in thermal equilibrium is given by the Brillouin function expressed in Equation 2.8 where $\mu$ is the magnetic permeability, and $k_{B}$ the Boltzmann constant. This, used in conjunction with relationship between polarization, $P_{T E}$, and the area under the NMR signal, $A_{T E}$, Equation 2.9, can be used to obtain a calibration constant, $C$, for use in determining the polarization of the target while it is being actively polarized via DNP.

$$
\begin{gathered}
P_{T E}=\tanh \left(\frac{\mu B_{0}}{k_{B} T}\right) \\
P_{T E}=C A_{T E}
\end{gathered}
$$

A Liverpool Q meter circuit [33] was used to measure the proton's nuclear magnetic resonance (NMR) response within the ${ }^{14} \mathrm{NH}_{3}$ target material. The output signal of the $\mathrm{Q}$ circuit and components with input frequency swept $\pm 0.4 \mathrm{MHz}$ from a central frequency approximately equaling the Larmor frequency of the proton in an applied magnetic field of $5 T$ is shown in Figure 2.27. As the target material absorbs or emits energy the inductance of the surrounding coil changes causing a dip or bump in the above mentioned plot. In addition to this inductance change, the signal contains a characteristic Q-curve [33] also shown in Figure 2.27 .

Several methods of subtracting the Q-curve from signal were tested, with the most reliably accurate one being subtraction of a baseline measurement. The baseline was taken with the magnetic field surrounding the target set so the Larmor frequency of the proton did not fall within the frequency sweeping range i.e. the coils surrounding the target had a constant impedance, see Figure.2.28(left) The resulting baseline subtracted data were then fit with a 3rd order polynomial to supplement the small variations of the baseline Figure 2.28(center).

The temperature used in the TE calculation was from a MKS Baratron3 liquid $\mathrm{He}$ 


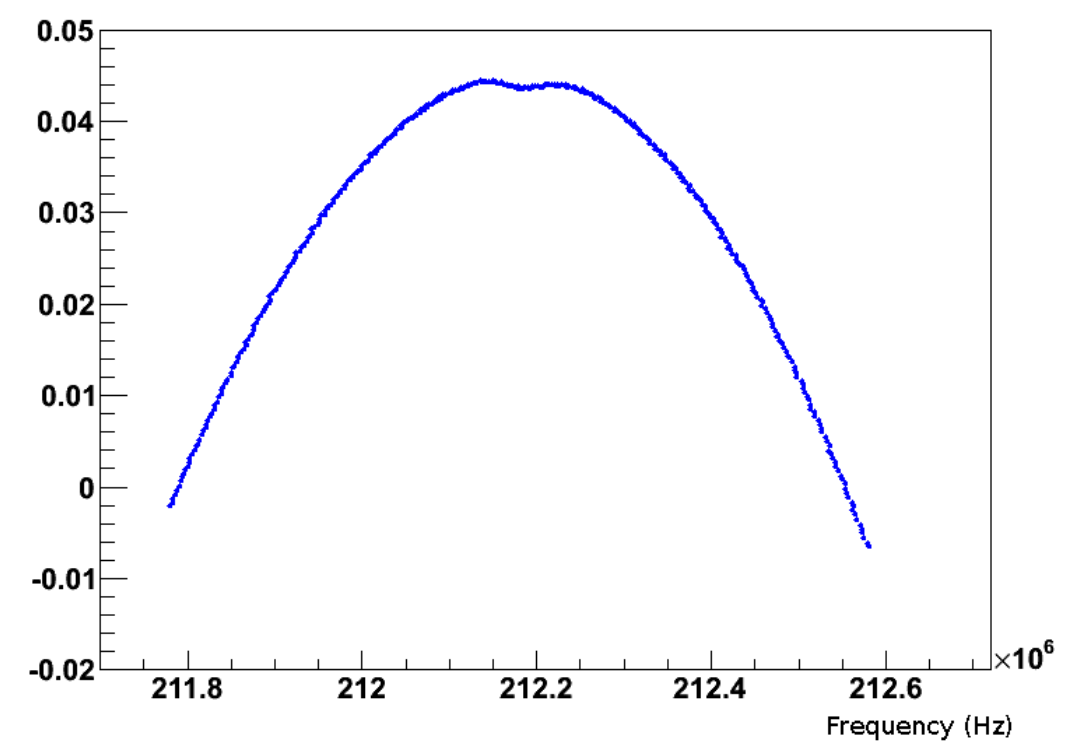

Fig. 2.27: Signal when target is at thermal equilibrium. The small dip in the Q-curve signal is due to the thermal equilibrium polarization of the protons in the target material.

probe. The readback of the temperature was delayed on the order of minutes, so the temperature used was the average readout over the course of a set of TE scans.

A normalized Gaussian function was used to determine the area of the background subtracted thermal equilibrium NMR curve. Figure 2.28(left). The agreement of the fit to the data fell within the noise level of the data.

TE's were taken periodically throughout the experiment. A total of 17 TE measurements were conducted in Run Periods A and B of the EG1-DVCS experiment. Each TE measurement involved taking many scans, with each scan averaging on the order of 1000 sweeps through the above mentioned frequency band. This procedure was done to minimize any noise from the $\mathrm{Q}$ meter circuit. The areas of the TE measurements were then used to determine the multiplicative calibration constant $C$. The calibration constants for all the TEs taken throughout EG1-DVCS run periods A and B are shown in Figure 2.29. 

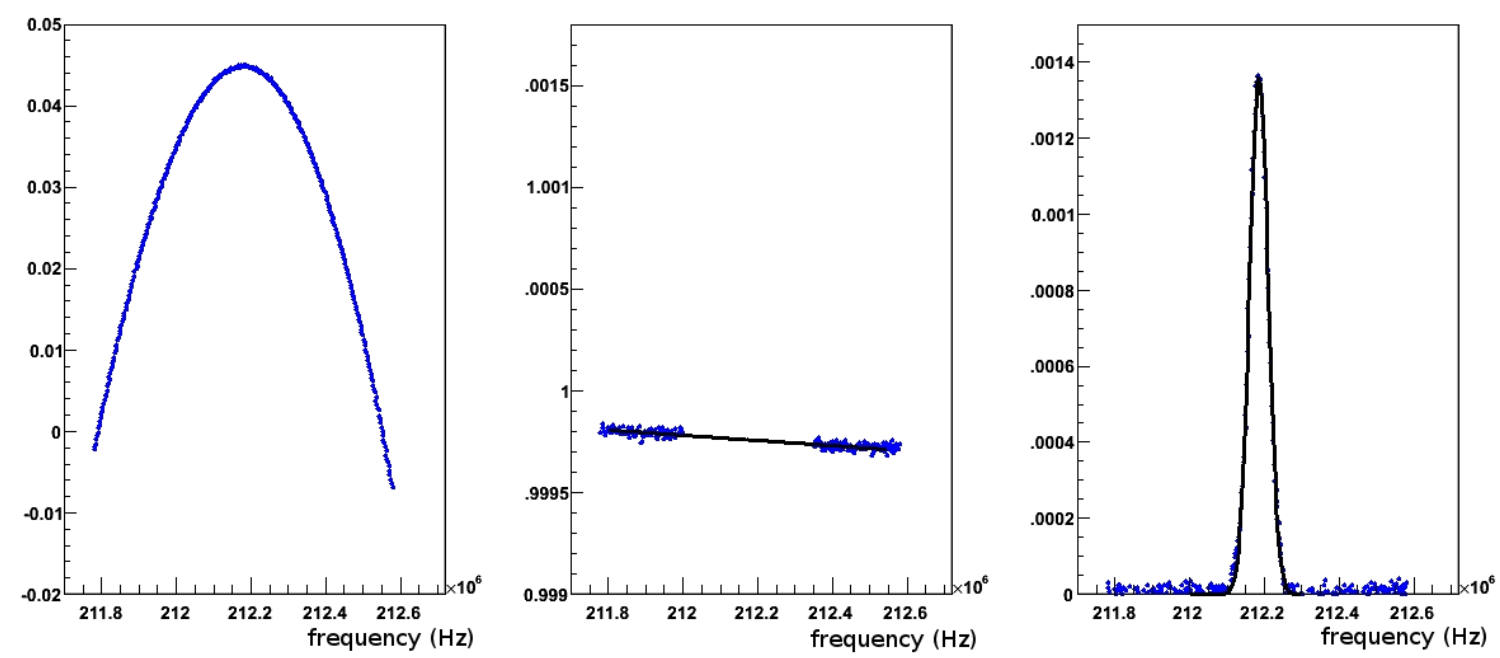

Fig. 2.28: Baseline scan (left), off-resonance signal minus baseline fitted with polynomial (center), signal minus baseline and polynomial, fitted with a Gaussian (right).
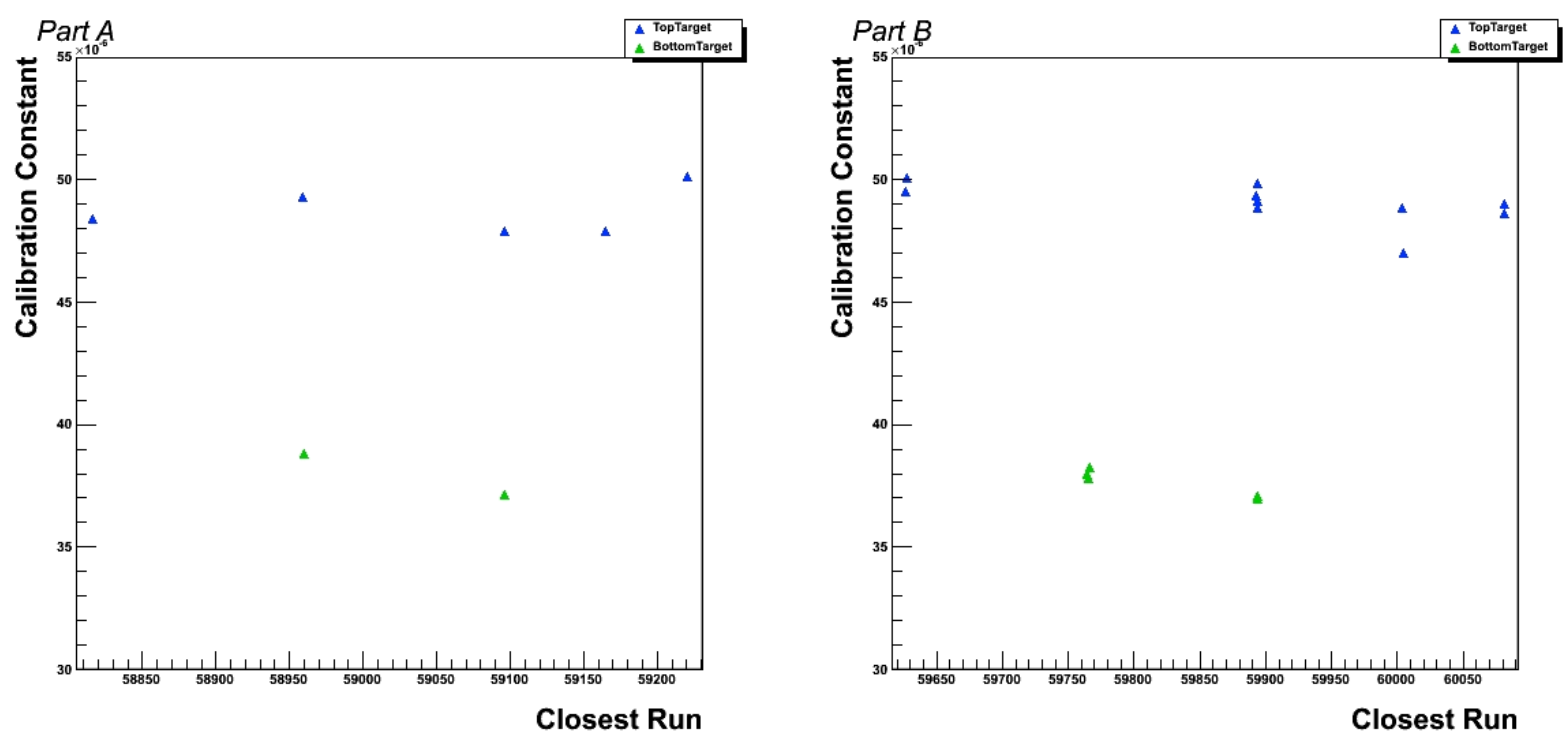

Fig. 2.29: Calibration constants for all TE measurements taken throughout EG1-DVCS Run Periods A and B. Blue points are from the top target cup, and green points from the bottom target cup. 


\subsubsection{Polarization Run-By-Run}

Once the calibration constants were determined, the areas of the NMR signals during active polarization were determined through a process of fitting the background subtracted NMR peak with a Gaussian and discretely summing the area within 4 sigma of the fitted Gaussian. This method was employed because the edges of the NMR peak were not represented by a Gaussian distribution, Figure 2.30. The polarization was then obtained by applying the associated multiplicative calibration constant determined by the TE measurements.

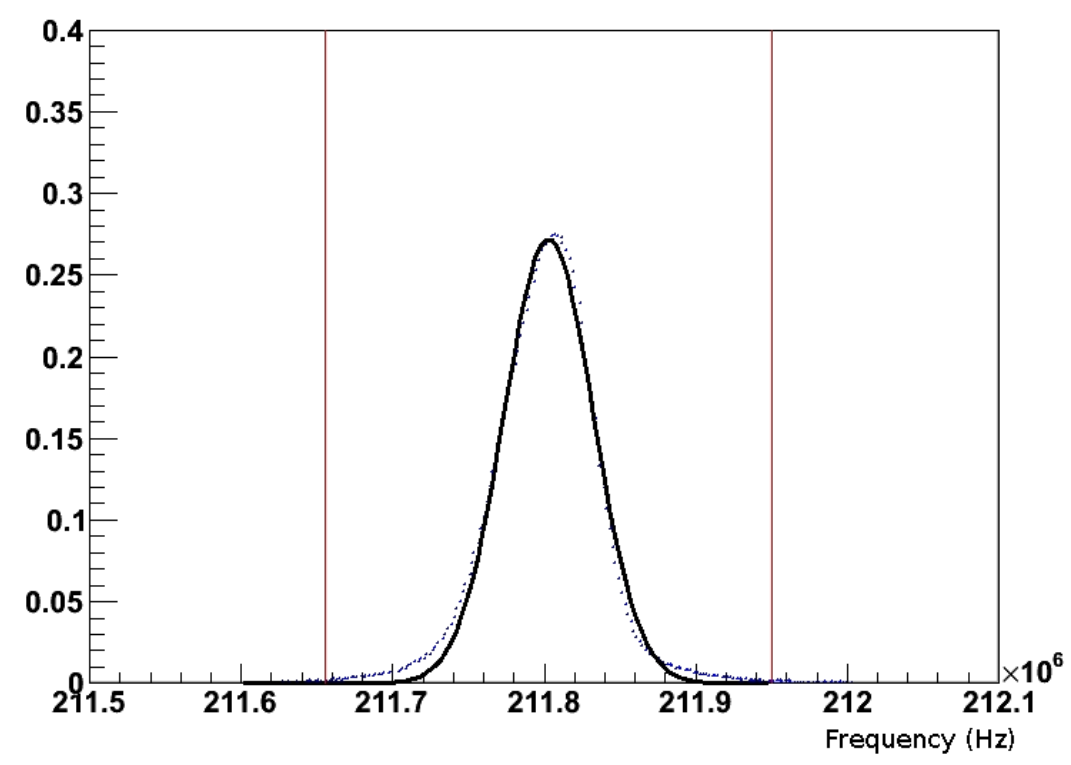

Fig. 2.30: Thermal equilibrium resonance signal minus baseline and polynomial fitted with a Gaussian. Red lines indicate 4 sigma from center.

The proton polarization during run periods A and B, plotted by run number, are shown in Figures2.31 and 2.32. 


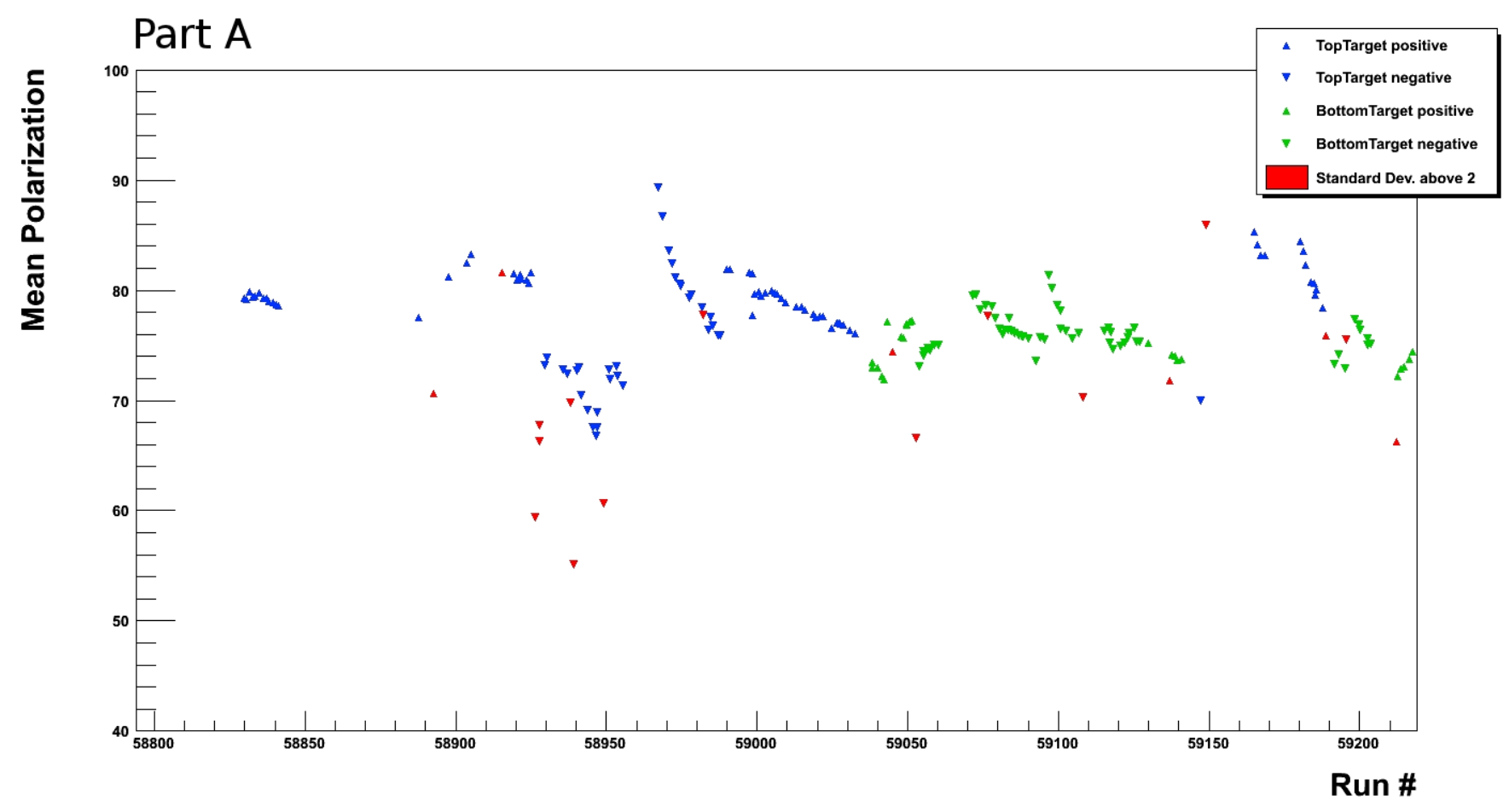

Fig. 2.31: Mean polarization plotted by run for EG1-DVCS Run Period A. Blue points are from the top target cup, and green points from the bottom target cup. The red points indicate when the standard deviation of polarizations in a run were greater than 2. This generally indicated a problem with the polarized target during the run, as can be seen in 2.33 . 


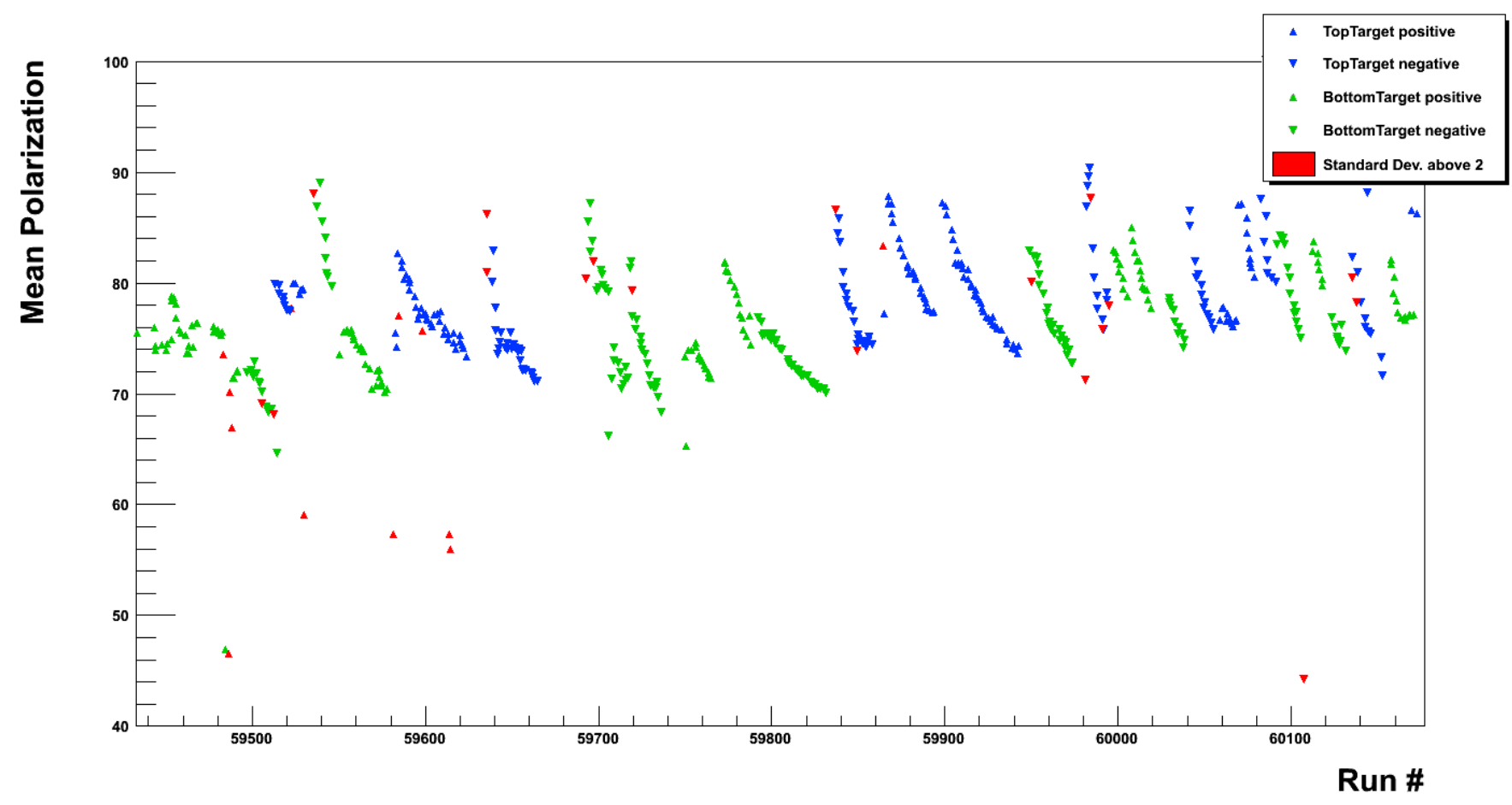

Fig. 2.32: Mean polarization plotted by run for EG1-DVCS Run Period B. Blue points are from the top target cup, and green points from the bottom target cup. The red points indicate when the standard deviation of polarizations in a run were greater than 2. This generally indicated a problem with the polarized target during the run, as can be seen in 2.33 . 


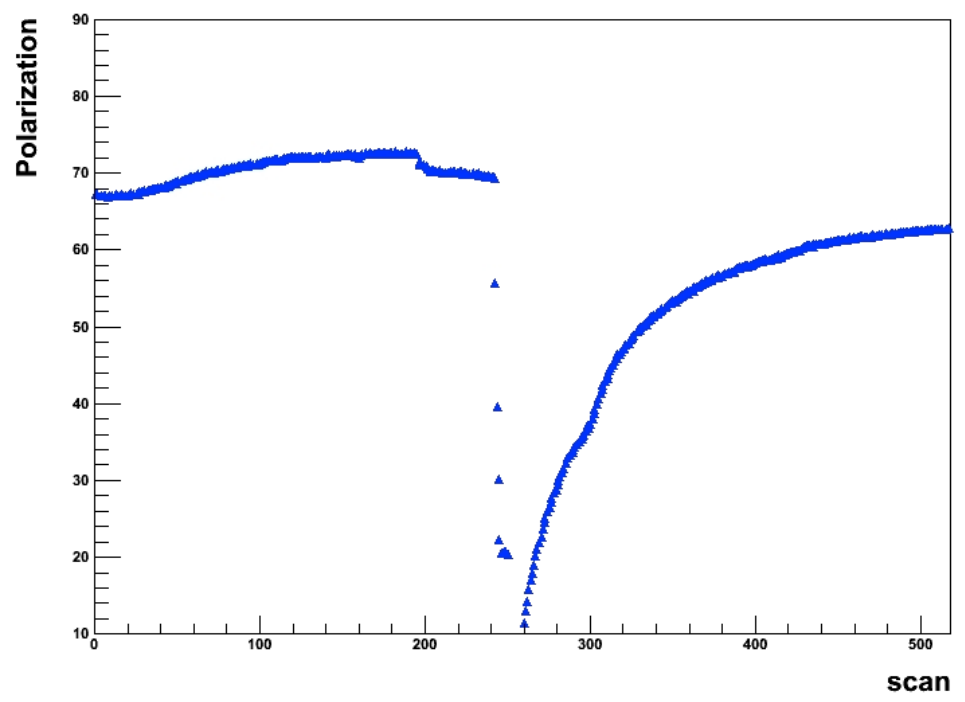

Fig. 2.33: Area of resonance peak vs scans taken throughout a problem run. The sudden drop in polarization occurred when the microwave generator mode-hopped. 


\section{Chapter 3}

\section{Data Selection}

\subsection{Run Selection}

Measurements of inclusive electron count rates (normalized by accumulated charge) are useful for checking the quality of the detector data. Count rates can change for a variety of reasons such as a change in torus current, beam energy, target material, or target polarization. Changes of those nature do not necessarily mean that there is a data quality problem. However, a sudden change in the count rate in an isolated sector can indicate a high voltage trip or detector problem. Uniform drops in count rate across all sectors can indicate a problem in the target such as a cryogenic leak, or problems in the Data Acquisition System (DAQ) or general electronics failure. A method was developed by A. Kim [34] to isolate data files that exhibit problematic changes in count rate for the EG1-DVCS data set. The procedure starts with grouping files based on the different run parameters of torus current, beam energy, target material, and target polarization. The inclusive electron rates for each sector in CLAS for each group are fit with a Gaussian function, and a cut on files that fall outside of $5 \sigma$ of the fit are discarded. The fitting and exclusion procedure is repeated until all "bad" files are eliminated. Table 3.1 shows the ratio of bad files to total number of files through each iteration of the above mentioned procedure. Figure 3.1 shows the distribution of electron rates for each data file of one of the run groups in Run Period A for each sector in CLAS, along with vertical and horizontal lines indicating Run changes and the distributions' $5 \sigma$ mark, respectively. 
("Bad" files)/(Total files)

\begin{tabular}{|l|l|}
\hline \hline After $1^{\text {st }}$ iteration & $1049 / 48710$ \\
After $2^{\text {nd }}$ iteration & $1054 / 48710$ \\
After $3^{\text {rd }}$ iteration & $1054 / 48710$ \\
\hline \hline
\end{tabular}

Table 3.1: Number of bad files after application of the run selection procedure [34].


Fig. 3.1: Electron rates for one group of runs, runs 58939 through 58956, from Run Period A, for each CLAS sector. The black vertical lines represent a change in Run number, the black horizontal line represents $5 \sigma$ from a Gaussian fit of each sector's rate distribution during the first iteration of the run selection procedure [34]. 


\subsection{Particle Identification}

This analysis looks at the exclusive DVCS channel where all scattered particles, e $p$ and $\gamma$, are detected. The following chapter details each selection cut used to identify particles.

\subsubsection{Electron Identification}

An electron candidate is first defined by time-of-flight, charge, and momentum using information mainly from the Scintillation Counters (SC) and Drift Chambers (DC). It is used as the trigger particle for an event in the CLAS detector. Additional selection cuts are needed to distinguish well defined "good" electrons from other light hadrons, mainly $\pi^{-}$, whose trajectory and time-of-flight overlap with electrons (at energies in the GeV). These additional selection cuts are done by exploiting the difference in interactions with matter between electrons and hadrons, which can be seen using combined information in the SC, DC, Cherencov Counters (CC) and Electromagnetic Calorimeters (EC). These additional selection criteria, including energy deposited in the EC, vertex position, timing, and fiducial cuts, are described below with a summary of the cuts in Table 3.2.

\subsubsection{Preliminary Data-Skim Cuts}

For electrons, the preliminary skim criteria mentioned in Section 2.5 are as follows:

1. Time based tracks with timing information in the EC and SC

2. charge $q \neq 0$

3. hits in matching DC, CC and EC sectors

4. momentum $\mathrm{p}>0.5 \mathrm{GeV} / \mathrm{c}($ see section 3.2.1.4)

5. etot $/ \mathrm{p}>0.12($ see section 3.2 .1 .9$)$

6. CC $\chi^{2}<0.15$ (see section 3.2.1.11) 


\begin{tabular}{|l|l|}
\hline Selection & Cut \\
\hline \hline charge & -1 \\
\hline IC Fiducial & see Figure: 3.2 \\
\hline EC Fiducial & \\
U & $40-400 \mathrm{~cm}$ \\
V & $0-360 \mathrm{~cm}$ \\
W & $0-390 \mathrm{~cm}$ \\
\hline Momentum & $>0.8 \mathrm{GeV} / \mathrm{c}$ \\
\hline Vertex & within $3 \mathrm{~cm}$ of nominal target position \\
\hline Timing & within 2 ns of Gaussian peak position \\
\hline Energy & EC inner energy $>0.06$ GeV \\
\hline
\end{tabular}

Table 3.2: Electron selection. Summary of electron identification cuts implemented in addition to the skim level cuts.

\subsubsection{IC Fiducial Cut}

Two fiducial cuts were applied for scattered electrons. The first is a cut on the shadow caused by the Inner Calorimeter (IC) on the Region 1 Drift Chambers. The IC housing is shown in Figure 3.2. Particles that go through the housing lose energy that is not accounted for, therefore they are not well reconstructed. To determine a proper fiducial cut $e^{+} / e^{-}$ratios were used [35]. In the area between the IC crystals and the IC housing, photons hit thick materials and convert to $e^{+} / e^{-}$pairs. Particles that hit the areas where the $e^{+} / e^{-}$ratio is high are not considered "good" electron candidates. The cut determined through the $e^{+} / e^{-}$ ratio study can be seen in Figure 3.2 for electrons. 

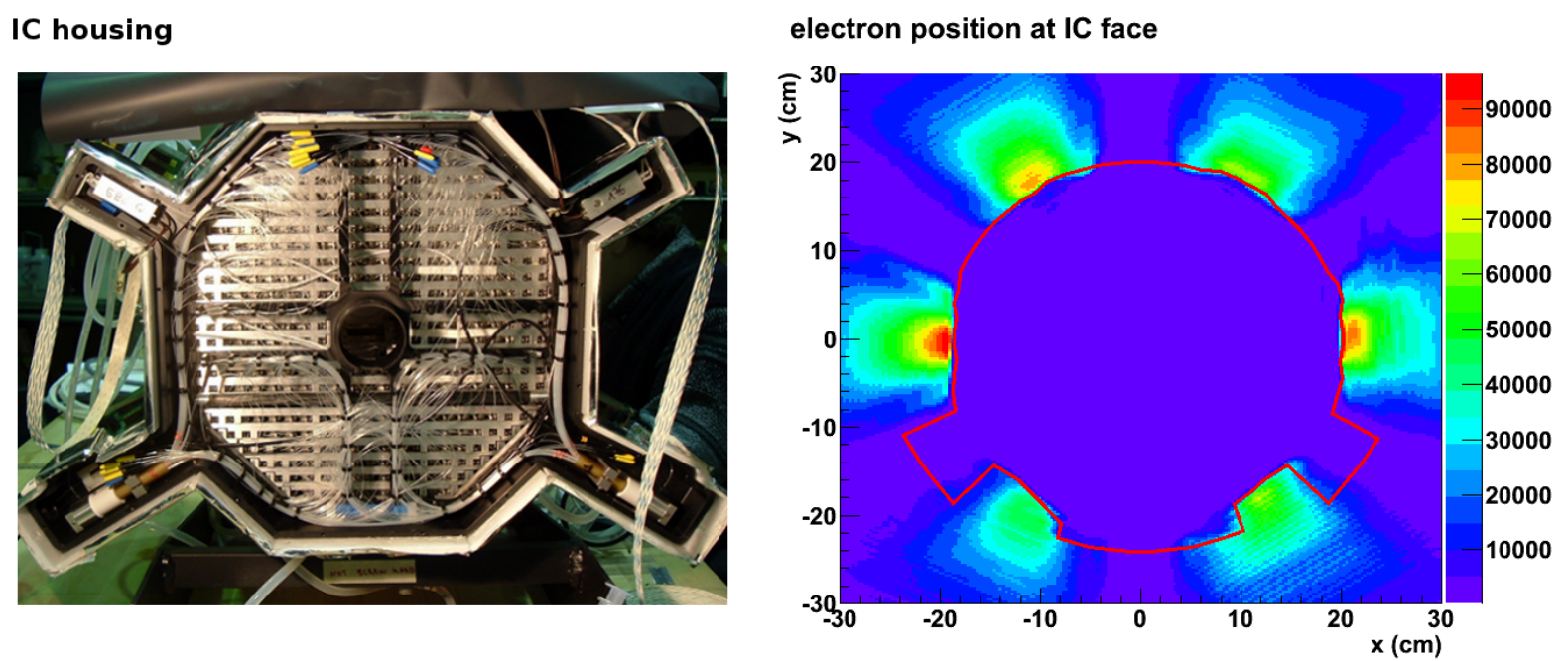

Fig. 3.2: Electron selection. Housing of the Inner Calorimeter (IC)(left), electron candidate particles' position at DC Region 1 (right). The red line indicates the IC fiducial cut. Any particles that are to the inside of the red line are considered "bad" electron candidates. 


\subsubsection{EC Fiducial Cut}

Electrons that hit the Electromagnetic Calorimeters (EC) near the calorimeters' edges do not loose all their energy in the detector because the shower is truncated. Therefore their energy is not properly reconstructed. A fiducial cut to reject particles that hit near the edges is determined by looking at the electron's position at the EC projected onto the $\mathrm{U}, \mathrm{V}$, and $\mathrm{W}$ planes of the EC (a description of the EC geometry is given in Section 2.4.0.5). Figure 3.3 shows the $\mathrm{U}, \mathrm{V}$, and $\mathrm{W}$ distributions with red lines indicating the cuts. The effect of the cut can be seen by looking at Figure 3.4 which shows the $\mathrm{x}-\mathrm{y}$ position of all electron candidates, left, and those electron candidates that pass the EC fiducial cut, right.
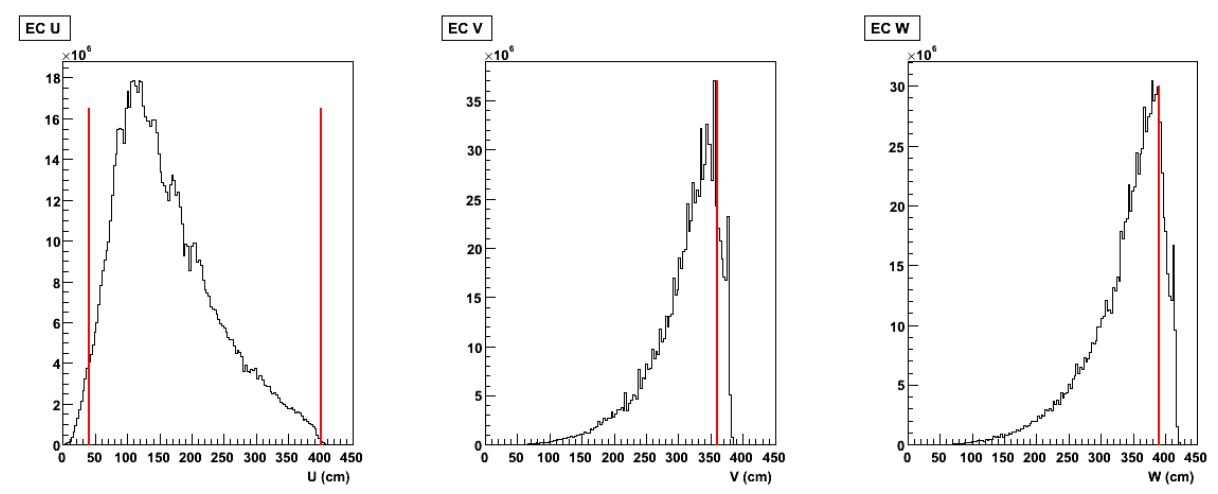

Fig. 3.3: Electron selection. Distribution of electron candidates in the U, V, and $\mathrm{W}$ planes of the EC. Applied cuts are shown in red. The effect of the cuts on the $\mathrm{x}-\mathrm{y}$ distribution of electrons at the EC can be seen in Figure 3.4. 

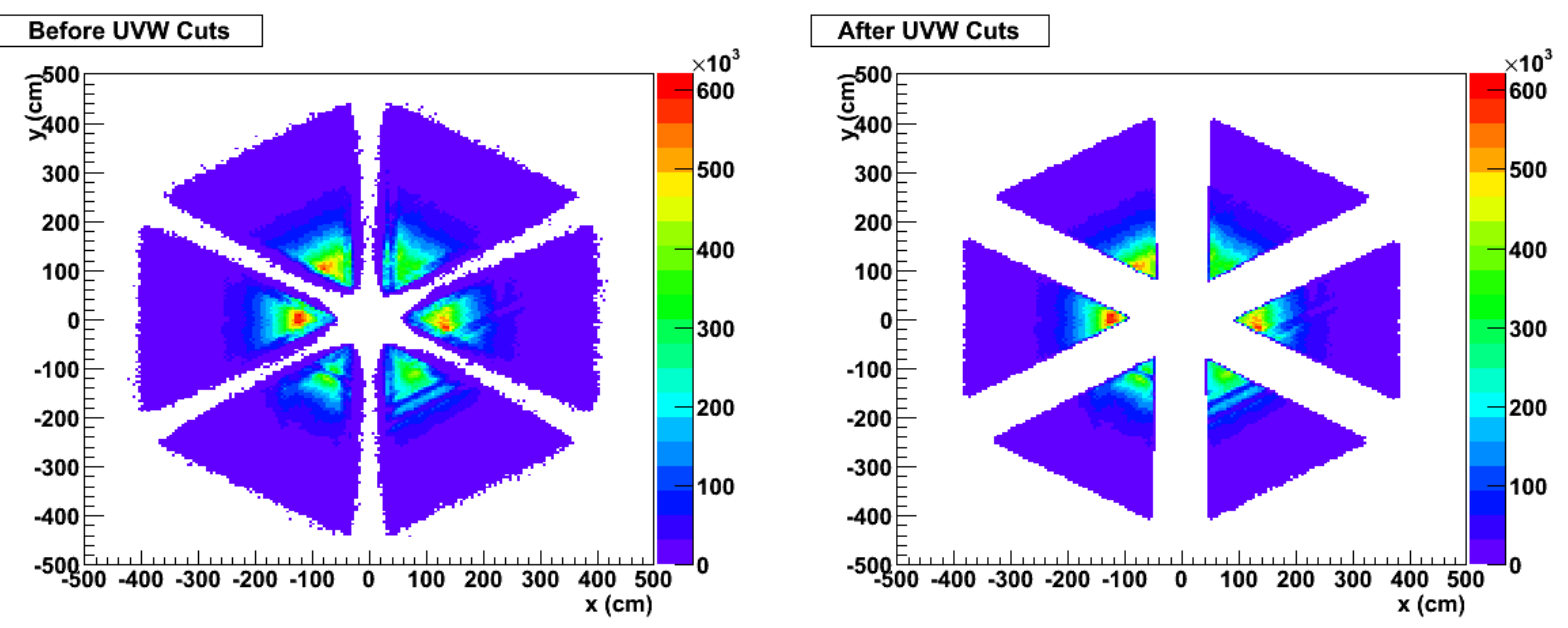

Fig. 3.4: Electron selection. EC x-y distribution of all electron candidates (left), and electron candidates that pass the EC fiducial cut (right). 


\subsubsection{Momentum Cut}

The EC is used to trigger events by detecting electrons above a set energy threshold. For a given threshold, one would expect the spectrum of detected electrons to cut off abruptly at the corresponding threshold energy. However, in practice, because of amplitude fluctuations of the EC response this cut is not sharp, which leads to distortions of the actual electron energy, $Q^{2}$, W, etc... at energies near the thresholds [36]. This effect was not corrected due to uncertainties in the EC output summing electronics. The distortion that results from the threshold at low energies can be seen by looking at the distribution of energy over momentum as a function of momentum, Figure 3.5. A minimum momentum cut was chosen to exclude electrons that fall below the trigger threshold. The data-skim applied a preliminary cut of $500 \mathrm{MeV}$, but for this analysis we found the optimal cut to be at $800 \mathrm{MeV}$ (just above the edge of distortion).

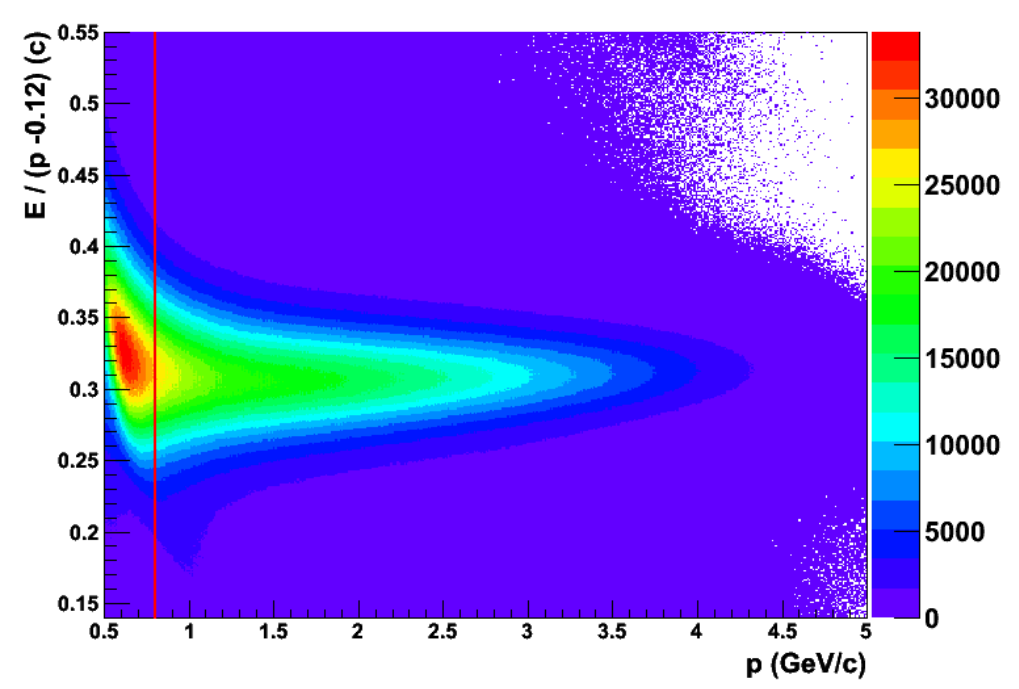

Fig. 3.5: Electron selection. Energy over momentum vs. momentum for electron candidates, red line indicates minimum momentum cut on electrons. 


\subsubsection{Vertex Cut}

A vertex cut was applied to the corrected z-vertex position of scattered electrons to reject events that take place outside of the target material. This was done after having applied the raster, angle and vertex corrections described in Sections 4.3 and Section 4.4. Figure 3.6 shows the electron's reconstructed z-vertex position minus the nominal target position, with red lines indicating the selection cut.

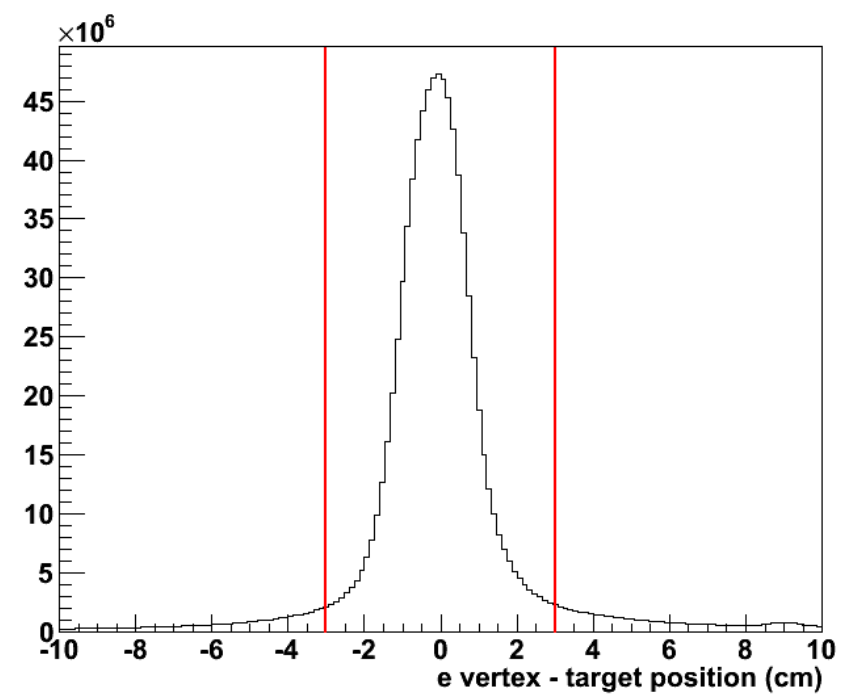

Fig. 3.6: Electron selection. Vertex distribution of electron candidates minus the nominal target position, red lines indicate cuts. 


\subsubsection{Timing Cut}

If a single particle hits both the CC and SC, the timing difference between the 2 hits differs by the track distance between the 2 detectors, and can be written as Equation 3.1 with $s c_{r}$ $\left(c c_{r}\right)$ as the path length from the target to the hit in the $\mathrm{SC}(\mathrm{CC})$, and $s c_{t}\left(c c_{t}\right)$ as the time of the hit in the $\mathrm{SC}(\mathrm{CC})$. The distribution of $\Delta t$ can be seen in Figure 3.7. The offset from 0 is attributed to calibration differences between the 2 detectors. The tail on the positive side of the Gaussian distribution is attributed to $\pi^{-}$, whose velocities are slower than c, and therefore Equation 3.1 does not hold. A cut of $2 \mathrm{~ns}$ from the electron peak position was applied to select "good" electron candidates.

$$
\Delta t_{c c-s c}=c c_{t}-\frac{c c_{r}}{c}-s c_{t}-\frac{s c_{r}}{c}
$$

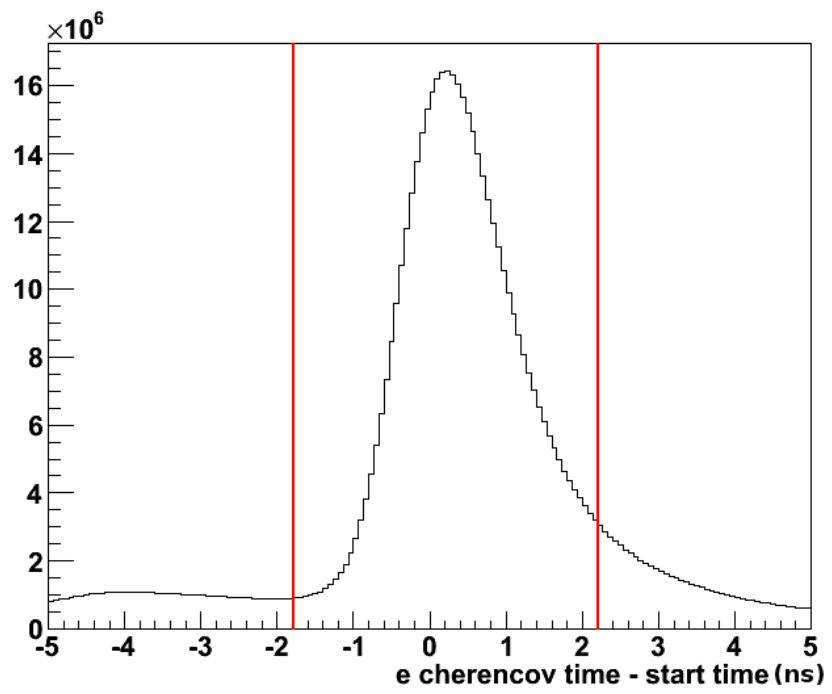

Fig. 3.7: Electron selection. Timing distribution of electron candidates, red lines indicate cuts. 


\subsubsection{Energy Cut}

$\pi^{-}$particles, whose mass is about $140 \mathrm{MeV}$, are substantially more massive than electrons $(0.5 \mathrm{MeV})$. As high energy $\pi^{-}$collide with heavy nuclei they lose energy in accordance with the Bethe-Bloch formula of energy $\operatorname{loss}^{1}$ 3.2. This formula dictates that the energy loss, $d E / d x$, decreases with increasing energy and reaches a minimum at $\beta \approx 0.96$. At speeds higher than this, the particles energy loss increases, but very slowly. Particles that are past this threshold of minimum energy loss are referred to as minimally ionizing particles. In the $\mathrm{GeV}$ energy range, $\pi^{-}$are minimally ionizing. Electrons on the other hand lose their energy primarily through bremsstrahlung radiation. At $\mathrm{GeV}$ energies the electron energy loss rate is directly proportional to the energy of the electrons in contrast to the nearly constant energy loss rate of $\pi^{-}$[38]. This can be seen in the ratio of energy lost in the Electrocalorimeter divided by the particle's momentum, Figure 3.9(left), which has 2 separate bands, one attributed to electrons, and one to $\pi^{-}$. To remove these $\pi^{-}$it was found sufficient to cut on the energy deposited in the inner part of the EC. $\pi^{-}$, being minimally ionizing, only loose around $30 \mathrm{MeV}$ of energy in the $15 \mathrm{~cm}$ thick inner layer of the Electromagnetic Calorimeter (EC). This can be seen in Figure 3.8. A cut at $60 \mathrm{MeV}$ was placed to eliminate $\pi^{-}$. The result on the energy momentum ratio distribution can be seen in Figure 3.9.

$$
-\frac{1}{\rho} \frac{d E}{d x}=4 \pi N_{a} r_{e}^{2} m_{e} c^{2} \frac{Z}{A} \frac{1}{\beta^{2}}\left[\ln \left(\frac{2 m_{e} c^{2} \gamma^{2} \beta^{2}}{I}\right)-\beta^{2}\right]
$$

\footnotetext{
${ }^{1}$ From reference [38] using $\beta=\frac{v}{c}$ and the assumption $W_{\max }=2 m_{e} c^{2} \gamma^{2} \beta^{2}$ (valid for high energies). $r_{e}$ is the classical electron radius, $m_{e}$ the electron mass and $N_{a}$ is Avogadro's number
} 


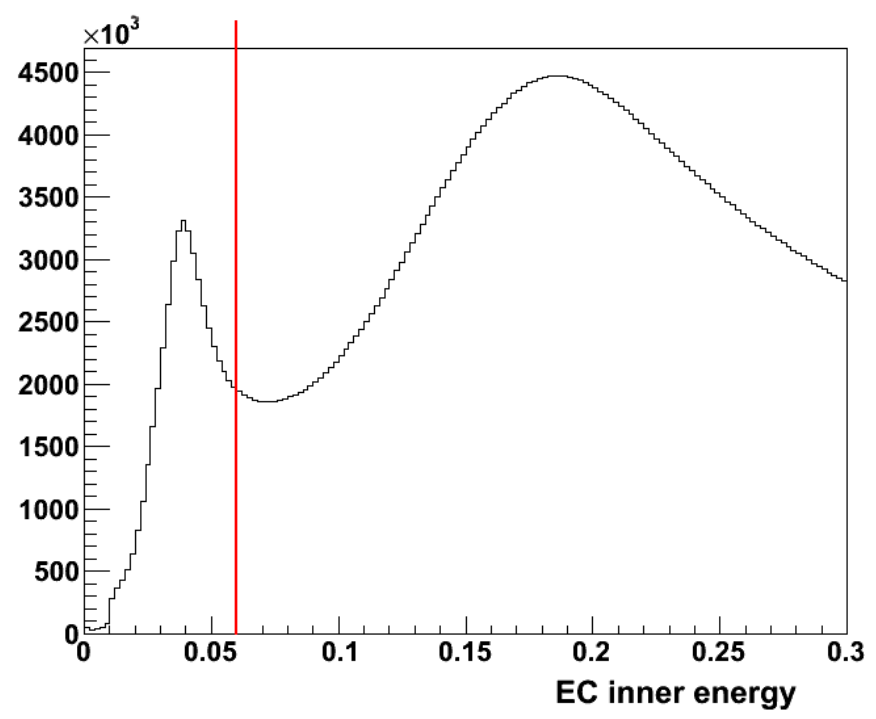

Fig. 3.8: Electron selection. Distribution of deposited energy in the inner region of the EC for electron candidates. The peak around $30 \mathrm{MeV}$ is attributed to $\pi^{-}$particles. The selection cut is shown in red, any particles that are to the left of the red line are considered "bad" electron candidates. 


\subsubsection{Other Electron Distributions}

There are several other cuts on electron distributions, besides the ones discussed above, that were studied but ultimately were not used in this analysis. Those include a cut on energymomentum ratio, number of photo-electrons produced in the $\mathrm{CC}$, and Cherencov mirror matching. This section will discuss the reasons for excluding those cuts.

\subsubsection{Energy Over Momentum}

As discussed in Section 3.2.1.7, the energy loss of an electron in the EC depends on the electron's energy, whereas for $\pi^{-}$the loss is only very weekly related to the $\pi^{-}$energy. This can be seen on the left side of Figure 3.9, which shows the ratio of total energy loss over momentum vs. momentum ${ }^{2}$. The lower band, which is presumed to be $\pi^{-}$, is negligible after the cuts described above were applied, shown in right plot of Figure. 3.9. For this reason a cut was not applied on the energy-momentum ratio.
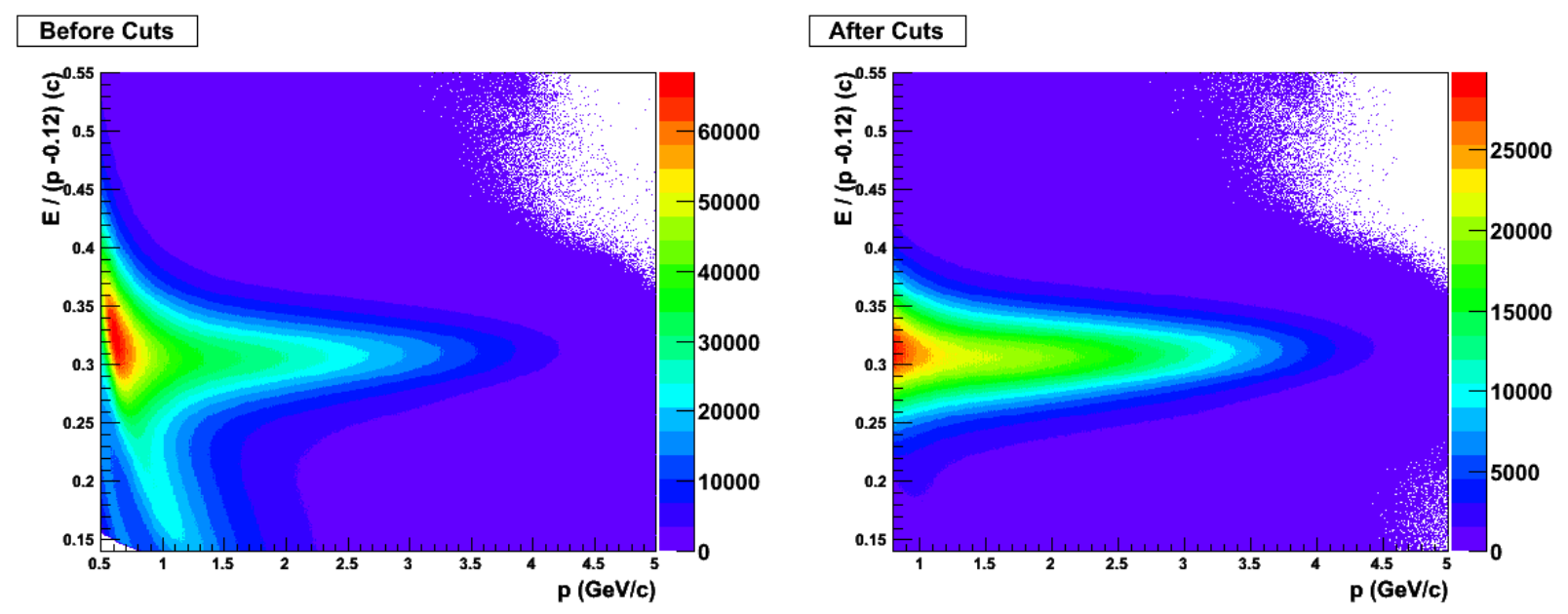

Fig. 3.9: Electron selection. Energy over momentum vs. momentum for electron candidates, before electron PID cuts (left) and after electron PID cuts (right).

\footnotetext{
${ }^{2}$ The denominator, (p-0.12) is used instead of $\mathrm{p}$ to have energy/momentum be independent of momentum. The electron looses a certain amount of energy before it gets to the EC, due to ionization energy loss. This occurs in the gas of the Cerenkov Counter, the material of the wire chambers, the EC support structure, and especially in the rather thick SC layer. This dependency can be corrected by applying a constant corrective term of -0.12 to the denominator [37].
} 


\subsubsection{Number of Photo-electrons}

The rate of photo production for negatively charged particles can be calculated as Equation. 3.3 [38], with $\alpha$ being the fine structure constant, $\alpha=\frac{1}{137}, \lambda$ the wavelength of the emitted light, and $n(\lambda)$ the refractive index of the medium.

$$
\frac{d^{2} N}{d \lambda d x}=\frac{2 \pi \alpha}{\lambda^{2}}\left(1-\frac{1}{\beta^{2} n(\lambda)^{2}}\right)
$$

$\pi^{-}$have velocities (and therefore $\beta$ ) considerably lower than electrons, so a smaller number of photo-electrons (nphe) will be produced in the CC for $\pi^{-}$as opposed to electrons. This can be seen on the left plot in Figure. 3.10. The large peak at around 1.2 photo-electrons is attributed mainly to $\pi^{-}$. There are also good electrons at those small values of nphe which are attributed to angular regions where the produced photoelectrons miss the CC photomultiplier tubes. For this reason a cut was not applied on the nphe distribution. The large peak around 2 photo-electrons, attributed mainly to $\pi^{-}$, is significantly reduced after the cuts described above were applied, shown in right plot of Figure. 3.10.
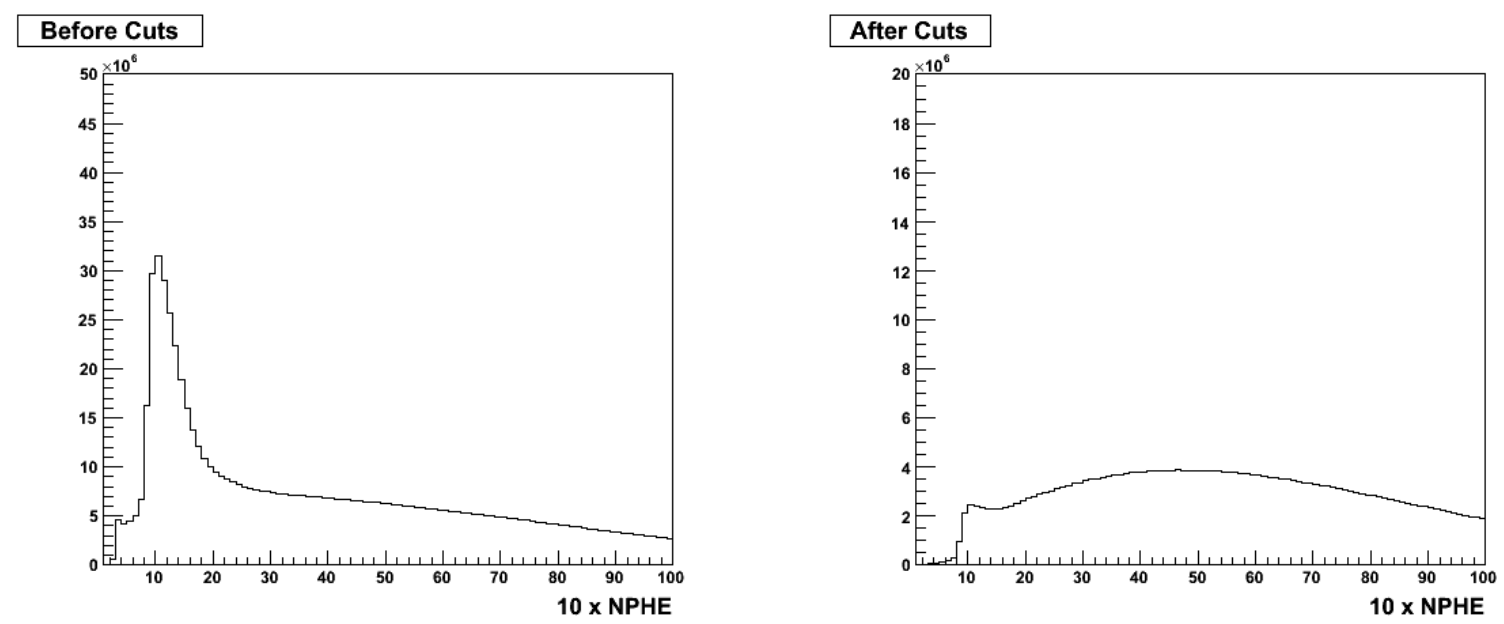

Fig. 3.10: Electron selection. Number of photo-electrons in the CC before (left) and after (right) electron PID cuts. 


\subsubsection{Cherencov Mirror Matching}

A new definition of the correlation between Cherencov counter mirror segment number and time-of-flight paddle number, $\chi^{2}$, was developed by Peter Bosted [39]. The original definition of $\chi^{2}$ in the CLAS reconstruction software was developed to work for particles whose z-vertex position is near CLAS center $(z=0)$. The EG1-DVCS target was positioned back from CLAS center to $z=-57.95 \mathrm{~cm}$ for part A, and $z=-67.97 \mathrm{~cm}$ for part B. The $\chi^{2}$ definition used in the EG1-DVCS analysis is given in Equation 3.4, with $M$ being the Cherencov mirror segment number and $P$ the associated SC paddle number.

$$
\chi^{2}=\frac{\left(1.1 M+0.003 M^{2}-P\right)^{2}}{80}
$$

In combination with the cuts used, cutting on the $\chi^{2}$ variable did not remove any other particles that would be considered $\pi^{-}$. However, a loose cut on $\chi^{2}$ for electrons was applied at the data-skim level at $\chi^{2}=0.15$. A study was done to see the effect of this loose cut on DVCS electrons. Out of 1,251,710 otherwise good electrons, 410 had a $\chi^{2}>0.15$, none of which passed DVCS exclusivity cuts (see section 3.5 for DVCS cuts). This skim-level loose cut was considered to contribute a negligible loss of electrons for this analysis. 


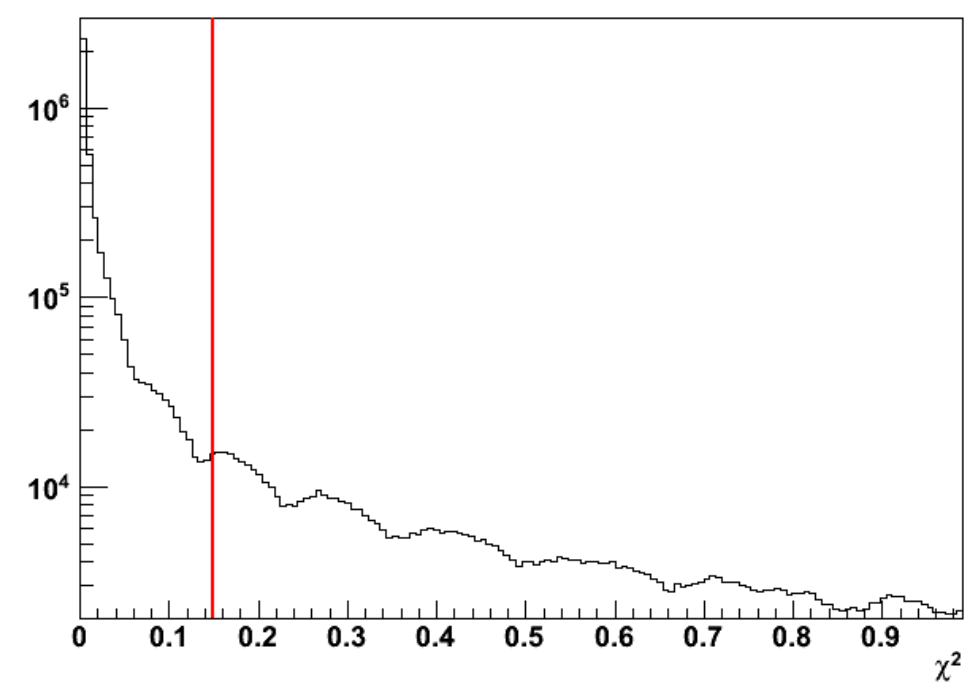

Fig. 3.11: Electron selection. The correlation between Cherencov Counter mirror segment number and time-of-flight paddle number, $\chi^{2}$ distribution for electron candidates. The red line indicates the skim-level $\chi^{2}$ cut applied to data. Any particles that are to the right of the red line are considered "bad" electron candidates. 


\subsubsection{Proton Identification}

Proton selection was done after identifying events with 1 "good" electron and at least one other particle with positive charge. Additional proton selection criteria are described below and include an IC fiducial cut, a z-vertex cut, and a momentum dependent $\beta$ cut. A summary of the cuts can be found in Table 3.3.

\begin{tabular}{|l|c|}
\hline Selection & Cut \\
\hline \hline charge & +1 \\
\hline IC Fiducial & see Figure 3.12 \\
\hline Vertex & within $4 \mathrm{~cm}$ of nominal target position \\
\hline$\beta$ & momentum dependent, see Figure 3.14 \\
\hline
\end{tabular}

Table 3.3: Summary of proton identification cuts

\subsubsection{IC Fiducial Cut}

The same fiducial cut described in Section 3.2.1.2 for electrons was applied for protons. Figure 3.12 shows the proton $\mathrm{x}-\mathrm{y}$ position at DC Region 1 both before(left) and after(right) the other proton selection cuts. 

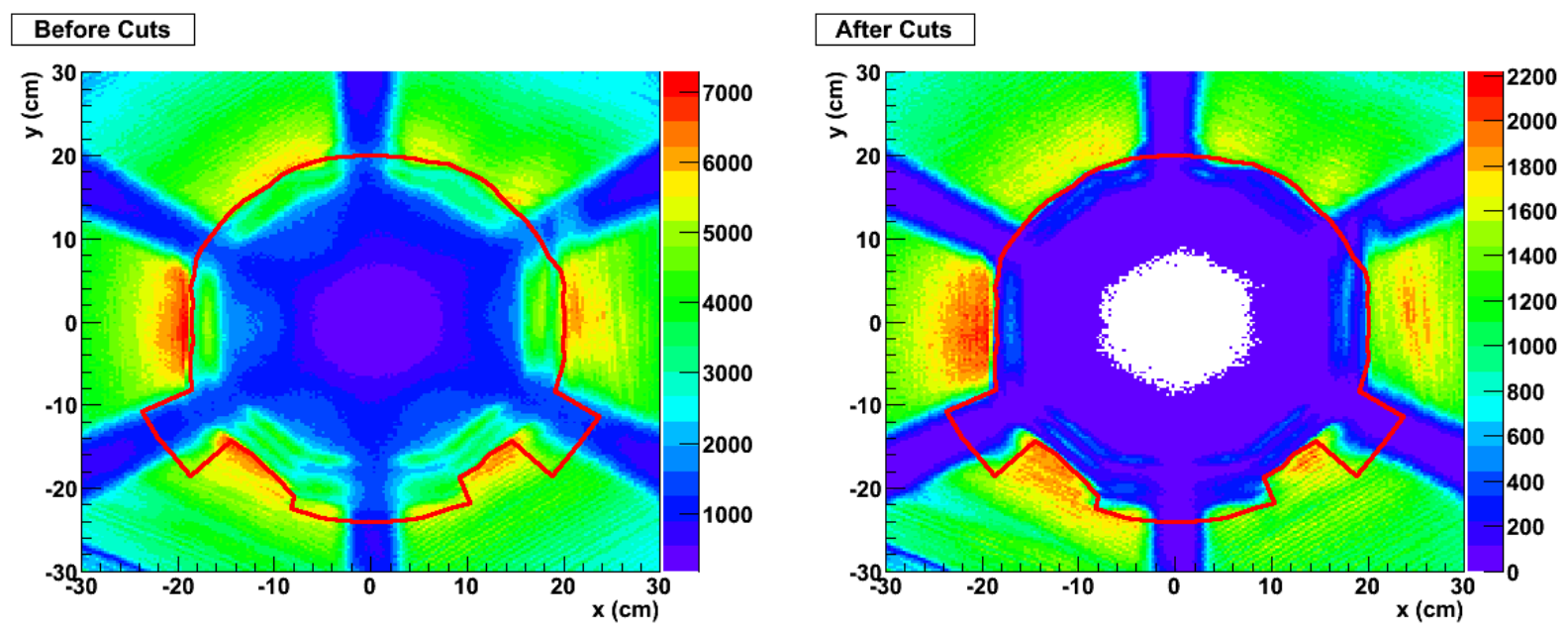

Fig. 3.12: Proton selection. Candidate proton position at DC Region 1 before (left) and after (right) other proton selection cuts. The red line indicates the IC fiducial cut. Any particles that are to the inside of the red line are considered "bad" proton candidates. 


\subsubsection{Vertex Cut}

A vertex cut, shown in Figure 3.13, was applied to the reconstructed z-vertex position of scattered protons to reject events that take place outside of the target material. This was done after having applied the raster and angular corrections described in Sections 4.3 and Section 4.4.

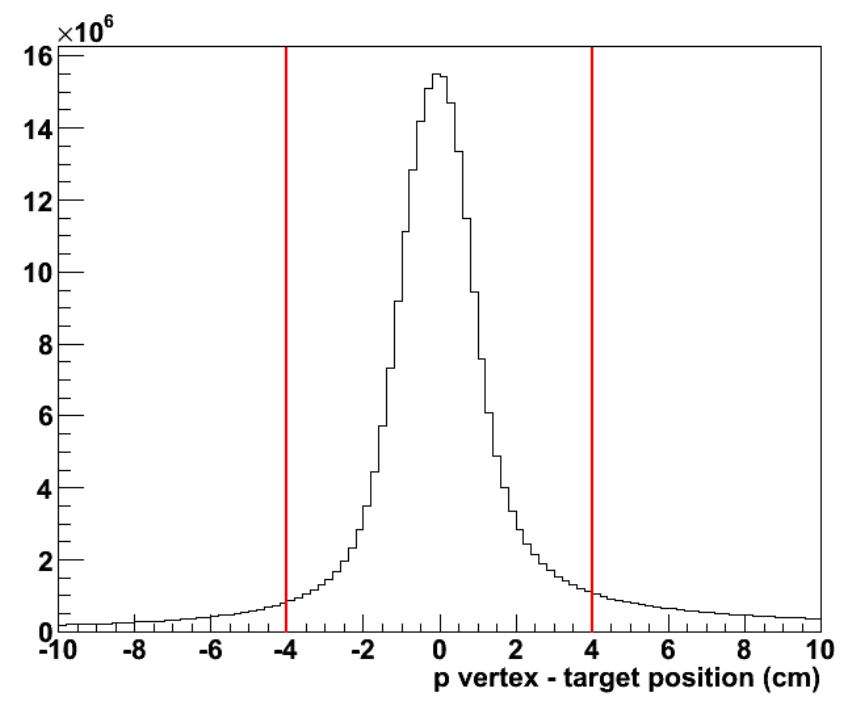

Fig. 3.13: Proton selection. Vertex distribution of proton candidates minus the nominal target position, red lines indicate cuts. 


\subsubsection{3 $\beta$ Cut}

The value of $\beta$ for a particle can be determined using the timing and positional information of the SC, as in Equation $3.5^{3}$. For a particle with mass, $\beta$ is related to its mass and momentum by Equation 3.6, so a momentum dependent $\beta$ cut was applied to select protons Figure 3.14.

$$
\begin{gathered}
\beta=\frac{s c_{r}}{\left(s c_{t}-t_{\text {event }}\right) * c} \\
\beta=\frac{p}{\sqrt{p^{2}+m^{2}}}
\end{gathered}
$$

To determine the selection cut, 1D $\beta$ distributions were fit with Gaussian functions in 11 small $(10 \mathrm{MeV})$ momentum bins. The distribution and the associated proton selection parameters for each of the 11 bins can be seen in Figure 3.15. The selection parameters of each bin were then plotted as a function of momentum, the black points in Figure 3.14, and fit with Functions 3.7. The resulting fit is shown as red lines in Figure 3.14.

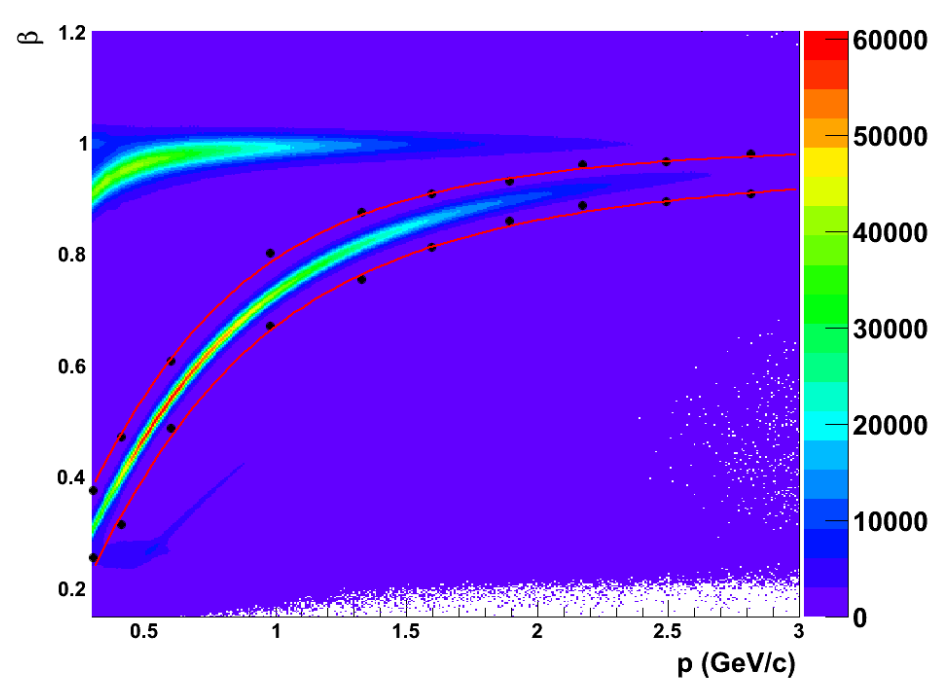

Fig. 3.14: Proton selection. $\beta$ distribution of proton candidates as a function of momentum. The distribution around $\beta=0.95$ is attributed to $\pi^{+}$particles. The black points correspond to the red lines shown in Figure 3.15. The red lines indicate the cut lines which are the result of a fit of the black points with Functions 3.7.

\footnotetext{
${ }^{3} s c_{r}$ is the path length from the target to the hit in the SC, $s c_{t}$ is the time of the hit in the SC, and $t_{\text {event }}$ is the start time of the event determined by the detection of the scattered electron
} 


$$
\begin{aligned}
& \beta_{\text {High }}=\frac{p}{\sqrt{p^{2}+0.938272^{2}}+[0]} * \frac{[1]+[2] * p}{1+p} \\
& \beta_{\text {Low }}=\frac{p}{\sqrt{p^{2}+0.938272^{2}}-[0]} * \frac{[1]+[2] * p}{1+p}
\end{aligned}
$$



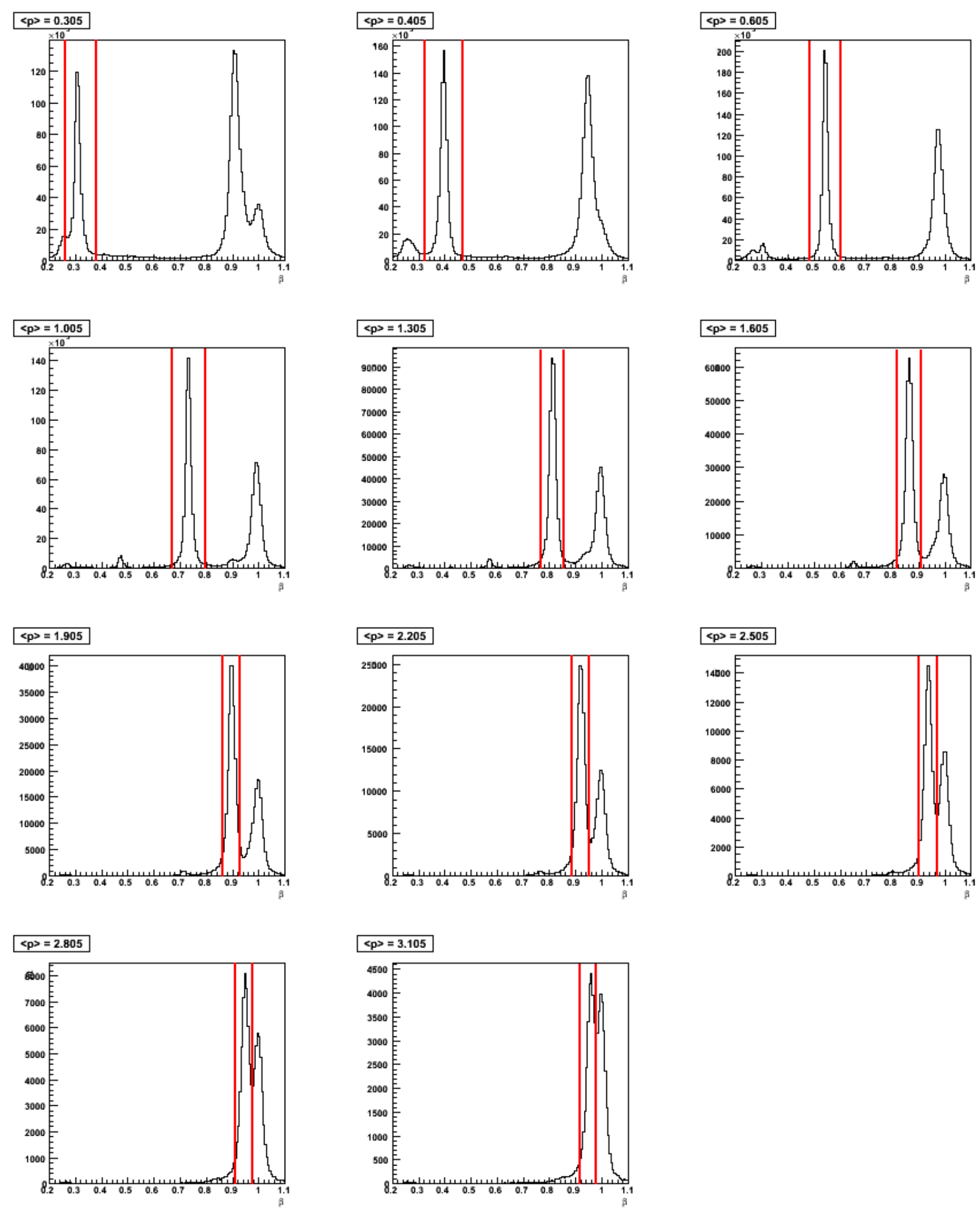

Fig. 3.15: Proton selection. $\beta$ distribution of proton candidates in $10 \mathrm{MeV}$ bins of momentum, red lines indicate proton selection parameters in each momentum bin. In addition to the peaks associated with protons there are also peaks associated with $e^{+}, \pi^{+}$and $K^{+}$particles. 


\subsubsection{EC Photon Identification}

Events with 1 "good" electron and at least 1 neutral particle detected in the EC were considered for EC photon selection. Additional EC photon selection criteria include fiducial cuts for both the EC and the shadow caused by the IC support frame, as well as a cut on measured $\beta$. This section describes the additional EC photon selection cuts with a summary of the cuts in Table 3.4.

\begin{tabular}{|l|c|}
\hline Selection & Cut \\
\hline \hline charge & 0 \\
\hline IC Fiducial & see Figure 3.16 \\
\hline EC Fiducial & \\
U & $40-400 \mathrm{~cm}$ \\
V & $0-360 \mathrm{~cm}$ \\
$\mathrm{~W}$ & $0-390 \mathrm{~cm}$ \\
\hline$\beta$ & $>0.92$ \\
\hline
\end{tabular}

Table 3.4: Summary of EC photon identification cuts

\subsubsection{IC Fiducial Cut}

A cut was applied on the position of photons at DC Region 1, similar to that applied to electrons and protons. However, for photons, the position was determined by tracing back the neutral tracks from the EC detectors to DC Region 1. The cut used can be seen in Figure 3.16. 


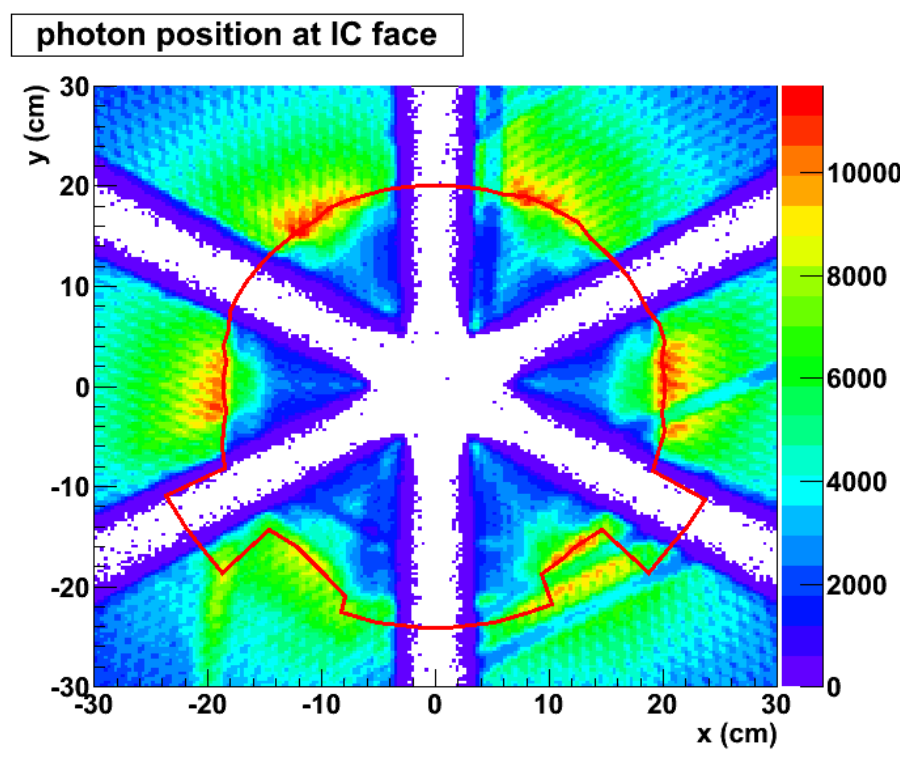

Fig. 3.16: EC photon selection. EC photon candidates $x-y$ position at DC Region 1. Red lines indicate the cut. Any particles that are to the inside of the red line are considered "bad" photon candidates. 


\subsubsection{EC Fiducial Cut}

The same EC fiducial cut applied to electrons is also applied to EC photons. This cut is used to reject particles that hit near the edges and is determined by looking at the photon's position at the EC projected onto the $\mathrm{U}, \mathrm{V}$, and $\mathrm{W}$ planes of the EC (a description of the EC geometry is given in Section 2.4.0.5). The effect of the cut is shown in Figure 3.17, which shows the $\mathrm{x}-\mathrm{y}$ position of EC photons at the EC detectors. The 6 distinct sections correspond to the 6 sectors of CLAS.
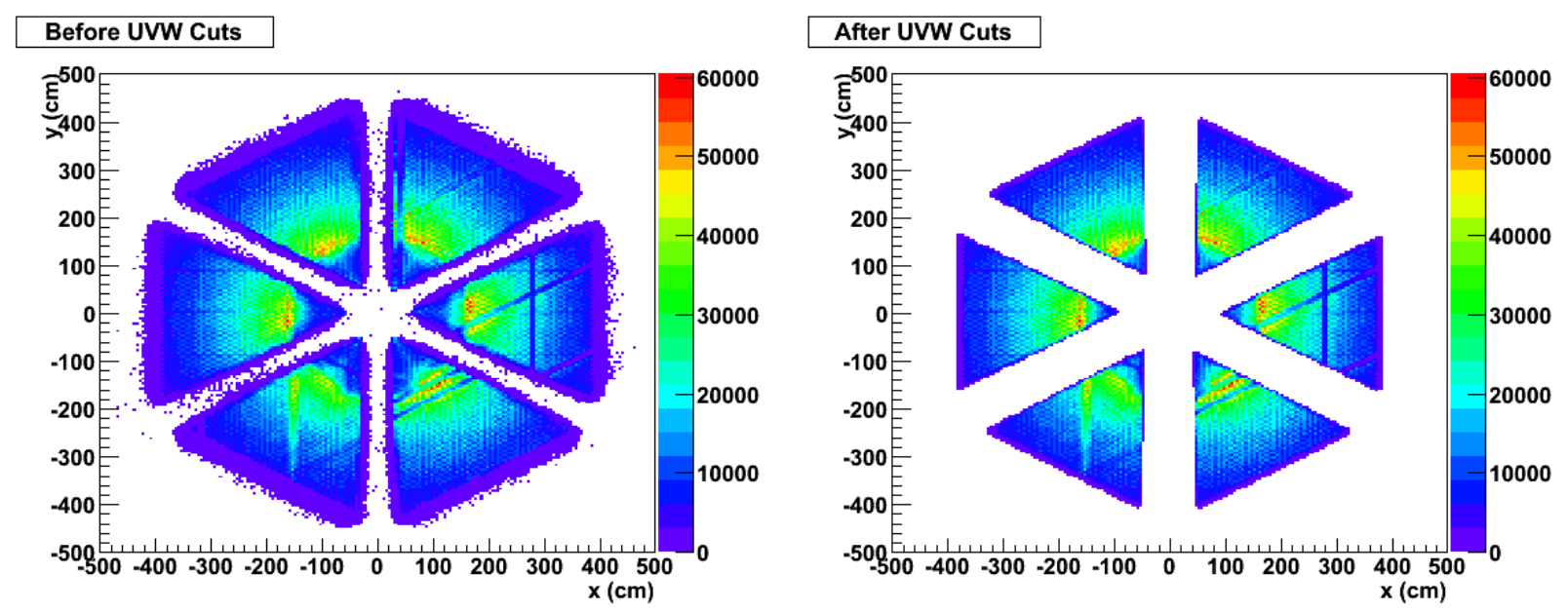

Fig. 3.17: EC photon selection. EC x-y distribution of all photon candidates (left), and photon candidates that pass the EC fiducial cut (right). 


\subsubsection{3 $\beta$ Cut}

Photons, which travel at $c$, have an energy independent $\beta$ of 1 . Due to the limitations of the detectors, the beta distribution of detected photons has a finite width, as shown in Figure 3.18. The value of $\beta$ for EC photons is determined using the timing and positional information of the EC for photons, as in Equation $3.8^{4}$. A cut on $\beta>0.92$ was applied to select well reconstructed EC photons.

$$
\beta=\frac{e c_{r}}{\left(e c_{t}-t_{\text {event }}\right) * c}
$$

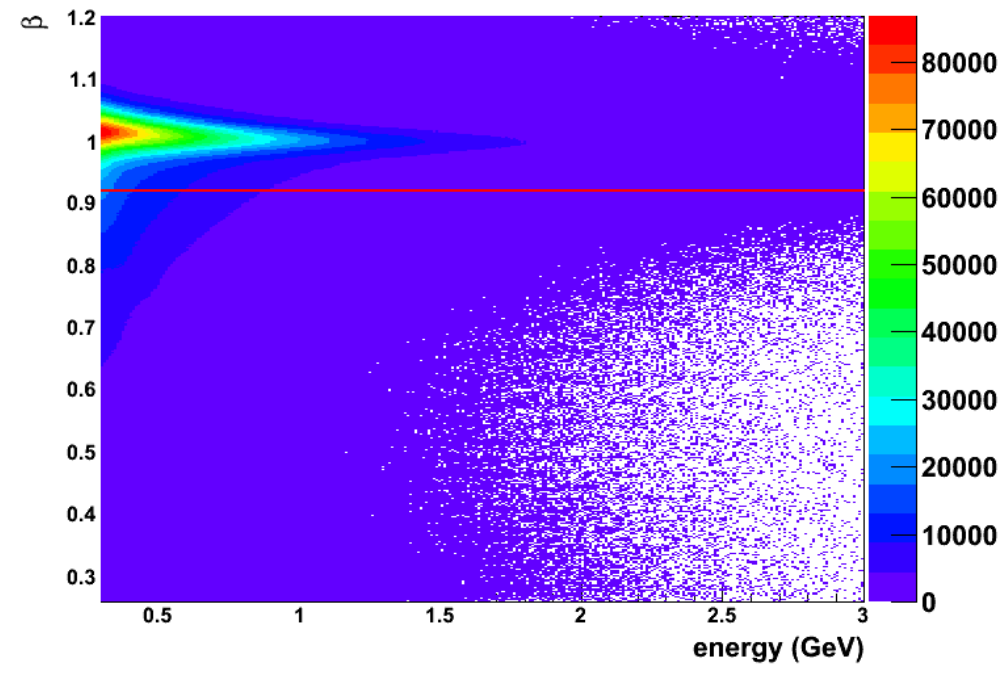

Fig. 3.18: EC photon selection. EC photon candidates $\beta$ distribution as a function of energy. Red line indicate the cut. Any particles that are below the red line are considered "bad" photon candidates.

\footnotetext{
${ }^{4} e c_{r}$ is the path length from the target to the hit in the EC, $e c_{t}$ is the time of the hit in the EC, and $t_{\text {event }}$ is the start time of the event determined by the detection of the scattered electron
} 


\subsubsection{IC Photon Identification}

\subsubsection{IC Fiducial Cut}

All particles hitting IC within the fiducial cut are considered "good" candidate photons (including Moller electrons). DVCS event selection is relied on for non-photon rejection. IC fiducial cuts, developed by F.X. Girod [40], are octagonal. Photons in which ALL of the conditions:

$$
\begin{aligned}
\frac{|x|}{s t e p X} & <3.25 \\
\frac{|y|}{s t e p Y} & <3.25 \\
\left|\frac{x}{\text { step } X}-\frac{y}{\text { stepY }}\right| & <3.25 \times \sqrt{2} \\
\left|\frac{x}{\text { step } X}+\frac{y}{\text { stepY }}\right| & <3.25 \times \sqrt{2}
\end{aligned}
$$

or:

$$
\begin{aligned}
\frac{|x|}{\text { stepX }} & >10.75 \\
\frac{|y|}{\text { stepY }} & >10.75 \\
\left|\frac{x}{s t e p X}-\frac{y}{\text { stepY }}\right| & >10.75 \times \sqrt{2} \\
\left|\frac{x}{\text { step } X}+\frac{y}{\text { stepY }}\right| & >10.75 \times \sqrt{2}
\end{aligned}
$$

are met are considered "bad" photon candidates and are rejected from this analysis. step $X$ $=1.346 \mathrm{~cm}($ step $Y=1.360 \mathrm{~cm})$ correspond to the horizontal (vertical) distance between the centers of two neighboring IC crystals. The values of 3.25 and 10.75 are set to reject reconstructed photon positions on the outside $1 / 4$ of the IC inner and outer edge crystals. The $\mathrm{x}-\mathrm{y}$ position of IC photons is shown in Figure 3.16, with the red line indicating the fiducial conditions listed in Equation 3.10. 


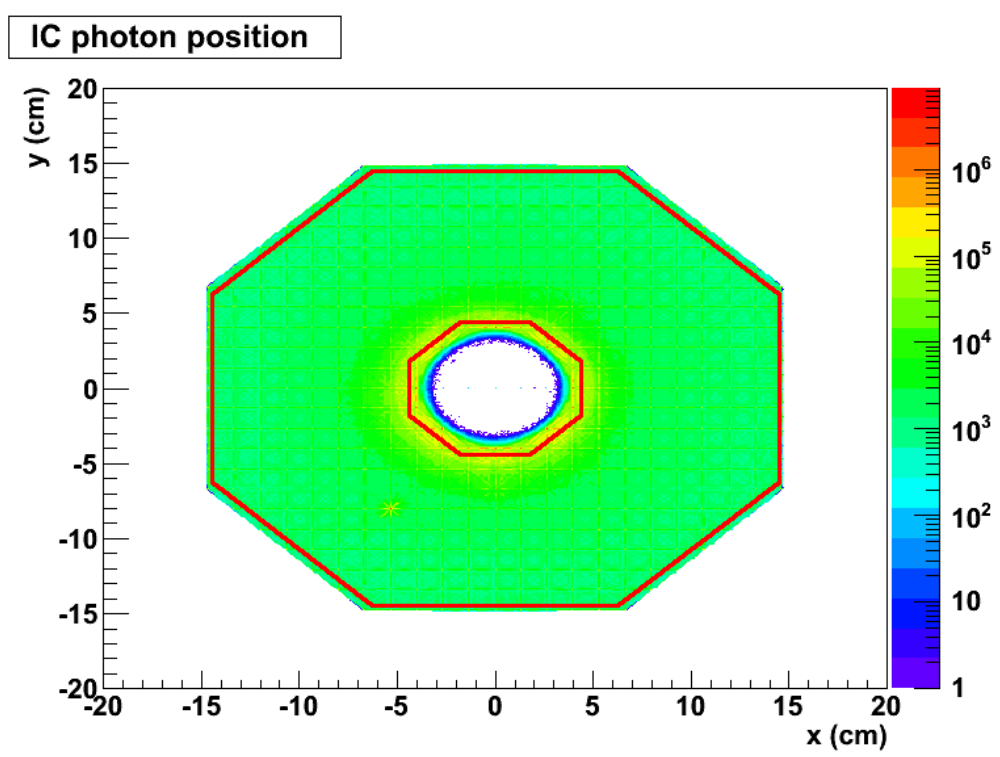

Fig. 3.19: IC photon selection. IC $x-y$ distribution of all IC photon candidates. Red lines indicate the cut. Any particles that are inside of the inner red line or outside of the outer red line are considered "bad" photon candidates. 


\subsection{Exclusive ep Elastic Selection}

Electron-proton elastic scattering is used in this analysis for the two following tasks:

- determination of electron beam energy (Section 4.1)

- determination of the proton target polarization (Section 4.2)

After selecting events with exactly one "good" electron candidate and one "good" proton candidate, and having applied the momentum and angle corrections described in Sections 4.5 and 4.4, further cuts needed to be applied to ensure selection of elastic events. Cuts on the following observables, or "exclusivity variables", were chosen to isolate elastic events and reduce the nuclear background coming from scattering on the nitrogen of the $\mathrm{NH}_{3}$ target:

- $0.858<W<1.018 \mathrm{GeV}$

- Missing energy for the $e p \rightarrow e p$ reaction, $E_{\text {miss }}<0.12 \mathrm{GeV}$

- Perpendicular component of the missing momentum, $p_{\text {perp }}<0.08 \mathrm{GeV} / \mathrm{c}$

- Longitudinal component of the missing momentum, $p_{\text {long }}<0.12 \mathrm{GeV} / \mathrm{c}$

- Initial electron energy difference, $-0.07<E_{0}-E_{\text {beam }}<0.06 \mathrm{GeV}$

where,

$$
E_{0}=M_{p}\left\{\left[\tan \left(\theta_{e} / 2\right) \tan \left(\theta_{p}\right)\right]^{-1}-1\right\}
$$

$M_{p}$ is the proton mass, and $\theta_{e}$ and $\theta_{p}$ are the polar angles of electron and proton, respectively. The first cut applied was on the elastic region of the $\mathrm{W}$ spectrum to eliminate the background from other channels. Figure 3.20 shows the W distribution; on the left plot with only particle identification cuts (Section 3.2), and on the right plot after all exclusivity cuts excluding the one on W. The other four distributions are shown in Figure 3.21. The plots on the left are the distributions with only particle identification and $\mathrm{W}$ cut applied, and the plots on the right are with all exclusivity cuts but the one on the variable plotted. 

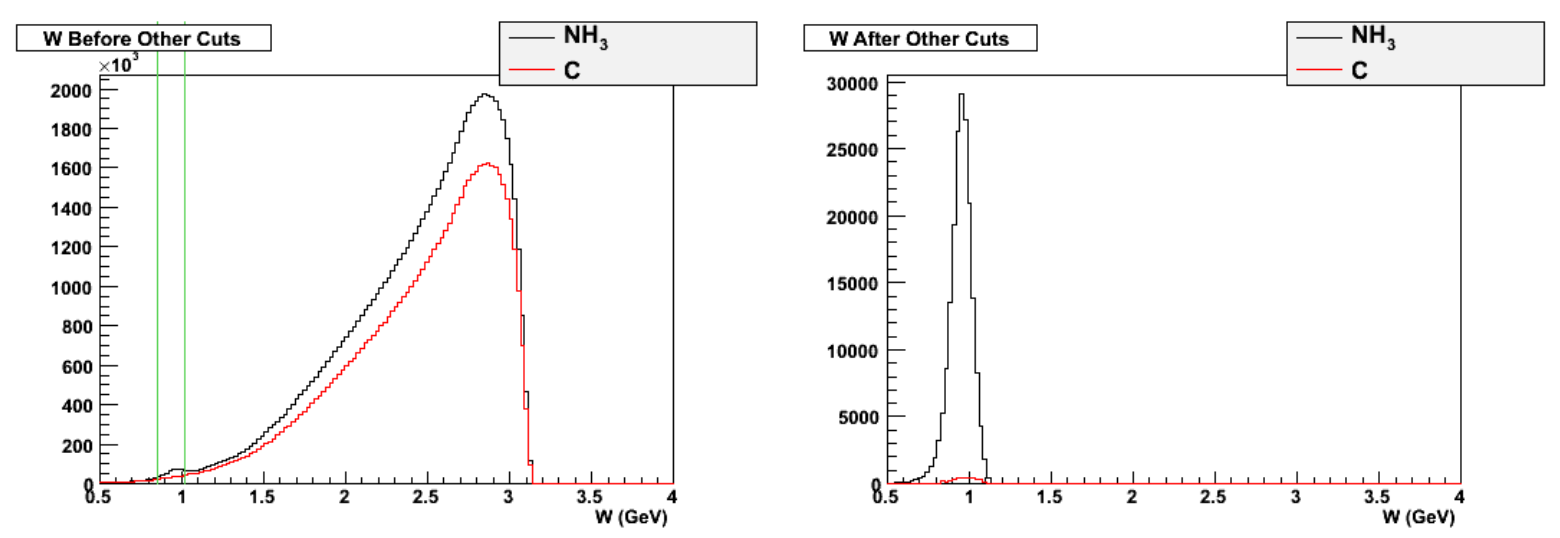

Fig. 3.20: Exclusive ep elastic analysis. W distribution (black: all $\mathrm{NH}_{3}$ data, red: charge scaled carbon data), before exclusivity cuts (left) and after all other exclusivity cuts (right). Green lines indicate cut values. 

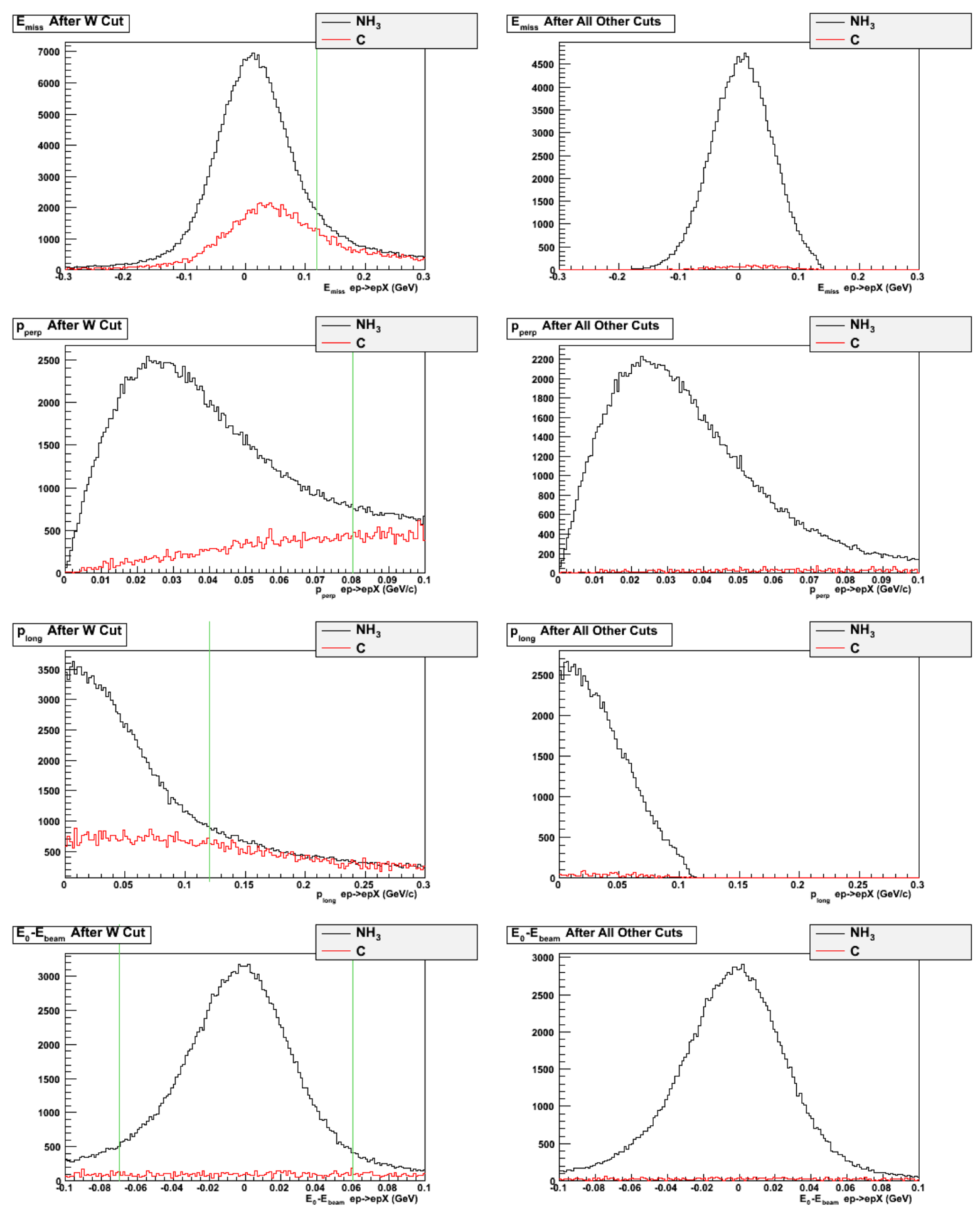

Fig. 3.21: Exclusive ep elastic analysis. Comparison of distributions (black: all $\mathrm{NH}_{3}$ data, red: charge scaled carbon data) with a cut on W (left column) and after all other exclusivity cuts (right column). From the first row down, missing energy $\left(E_{\text {miss }}\right)$, perpendicular component of the missing momentum $\left(p_{\text {perp }}\right)$, longitudinal component of the missing momentum $\left(p_{\text {long }}\right)$, and the difference between measured initial electron energy $E_{0}$ and electron beam energy $E_{\text {beam }}$. The green lines indicate cut values. 


\subsection{Monte-Carlo Simulations}

Monte-Carlo simulations are used in this analysis for the three following tasks:

- determination of momentum corrections for electrons and protons (Section 4.5)

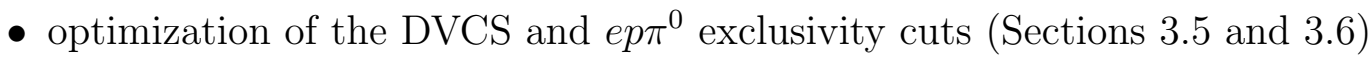

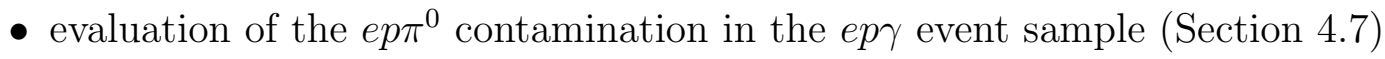

Two sets of generated events, DVCS/Bethe-Heitler (Section 3.4.1) and ep $\pi^{0}$ (Section 3.4.2) were put through GSIM, a GEANT3 based simulation program to simulate the response of the CLAS spectrometer. GSIM was modified to include the geometry of the EG1-DVCS polarized target, the different target-IC relative positions, shieldings and superinsulations, and beam energies between run periods A and B. With GSIM, particles are traced through the target region and detectors, hits are generated, and a simulated data file is produced.

The output from GSIM contains reconstructed events assuming perfect resolution for each detector. To better match the working conditions of the CLAS detector, after the events are processed with GSIM, they are then processed through the GSIM Post Processor (GPP) which smears the TOF times and the drift chamber DOCA. This is done by adding a pseudo-random time from a Gaussian distribution to each detector. GPP also simulates "dead" detector elements, such as dead wires in the drift chambers, and bad TOF scintillator paddles. Figure 3.22 shows the comparison obtained for the DC-wire efficiency for the data (top) and the Monte-Carlo simulation after GPP (bottom), for Sector 1. Similar matches were obtained for the other sectors, and the full study can be found in [41]. Once the data is put through GPP, it is processed using the same reconstruction program as the real data, user_ana. What results is a file that contains both the simulated event information and the GSIM/GPP/user_ana reconstructed event information. 


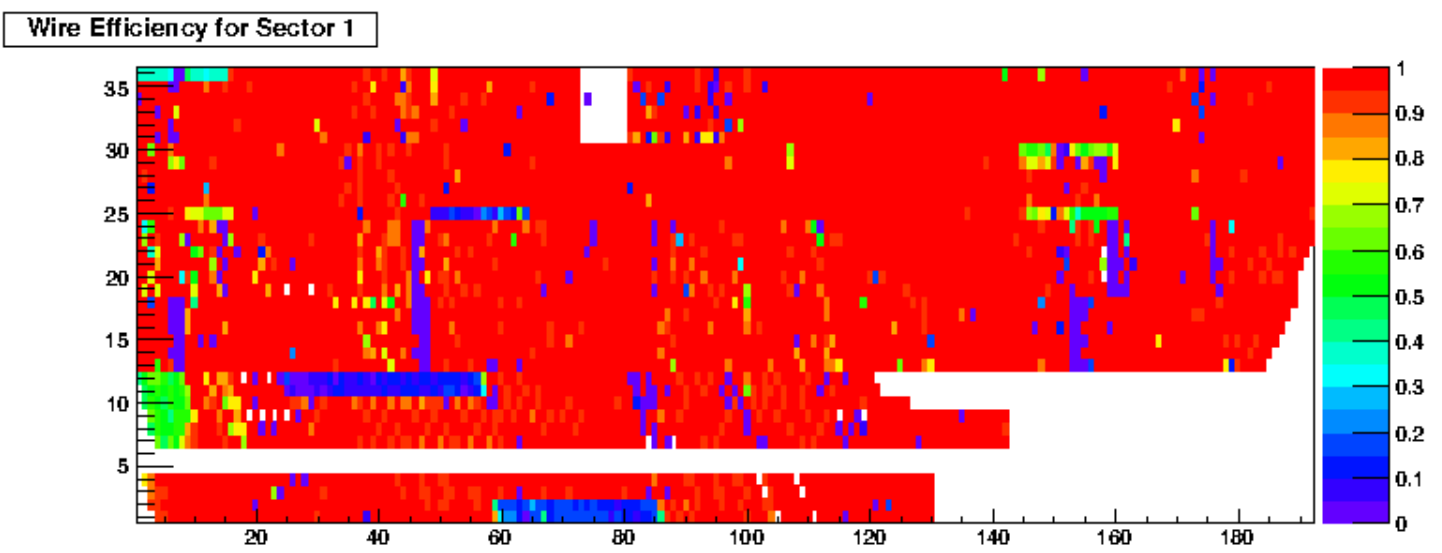

Wire Efficiency for Sector 1

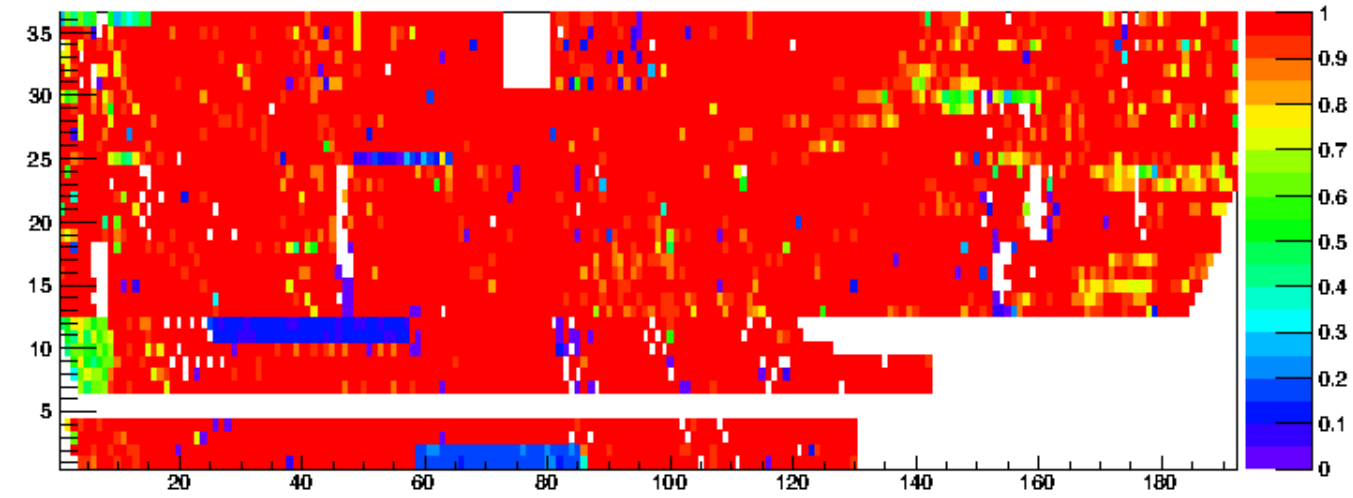

Fig. 3.22: Comparison of the efficiencies (on the $\mathrm{z}$ axis) of $\mathrm{DC}$ wires, for Sector 1 . The layer and the wire numbers are plotted on the $\mathrm{y}$ and $\mathrm{x}$ axes, respectively (a description of the DC geometry is given in Section 2.4.0.2). The EG1-DVCS data (Run Period A) are shown in the top plot and the Monte-Carlo simulation, after GPP, in the bottom [41]. 


\subsubsection{DVCS simulation}

DVCS/Bethe-Heitler events were generated using the "dvcsgen" code, which produces ep $\gamma$ events according to the formalism of Belitsky et al. [42]. The original code was modified in order to have the interaction vertex distributed over an ellipse of dimensions equal to those obtained with the raster pattern of EG1-DVCS and a cut on the minimal cone angle between the beam and the real photon, as well as on the one between the scattered electron and the real photon, $\theta_{e \gamma}>1.95^{\circ}$ was also imposed, in order to avoid the sharp singularities of the Bethe-Heitler process [1].

Figures 3.23 - 3.26 show comparisons of the distributions of the relevant kinematic variables for the data (left column) and the Monte-Carlo simulation (right column) (PID cuts (Section 3.2) and DVCS exclusivity cuts (Section 3.5) were applied for both the data and the Monte-Carlo). The difference between data and $\mathrm{MC}$ in the relative rates of IC and EC events is thought to be due to the fact that the data are not only pure DVCS/BetheHeitler events, but are contaminated by exclusive $\pi^{0}$ events. This is verified in Section 3.5.2 where the $e p \pi^{0}$ contamination was found to be stronger in the EC topology. 

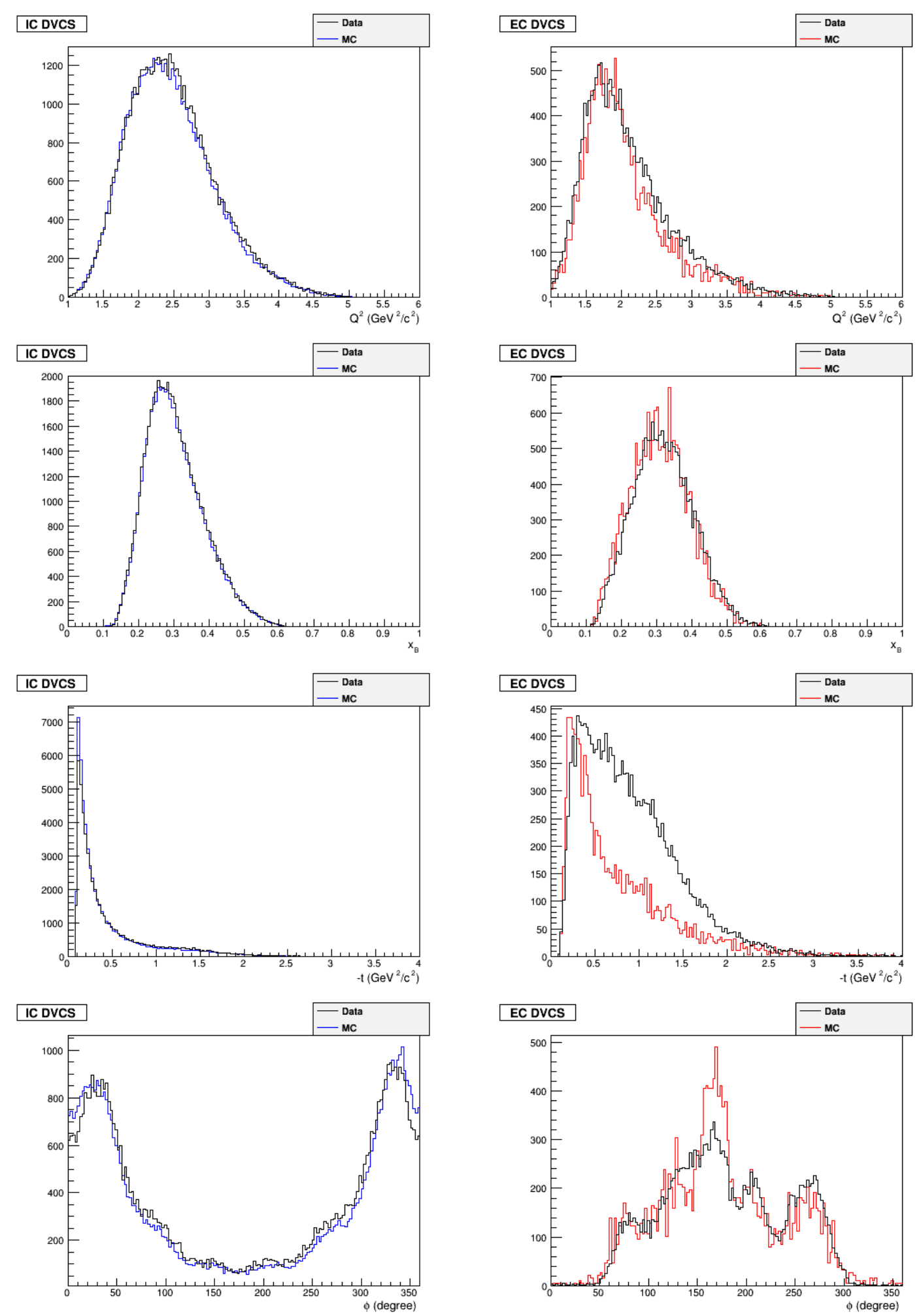

Fig. 3.23: DVCS channel. Comparison of data and Monte-Carlo simulation for DVCS events with the photon detected in the IC (left) and EC (right). From the first row down, $Q^{2}, x_{B},-t$ and $\phi$ are plotted. 

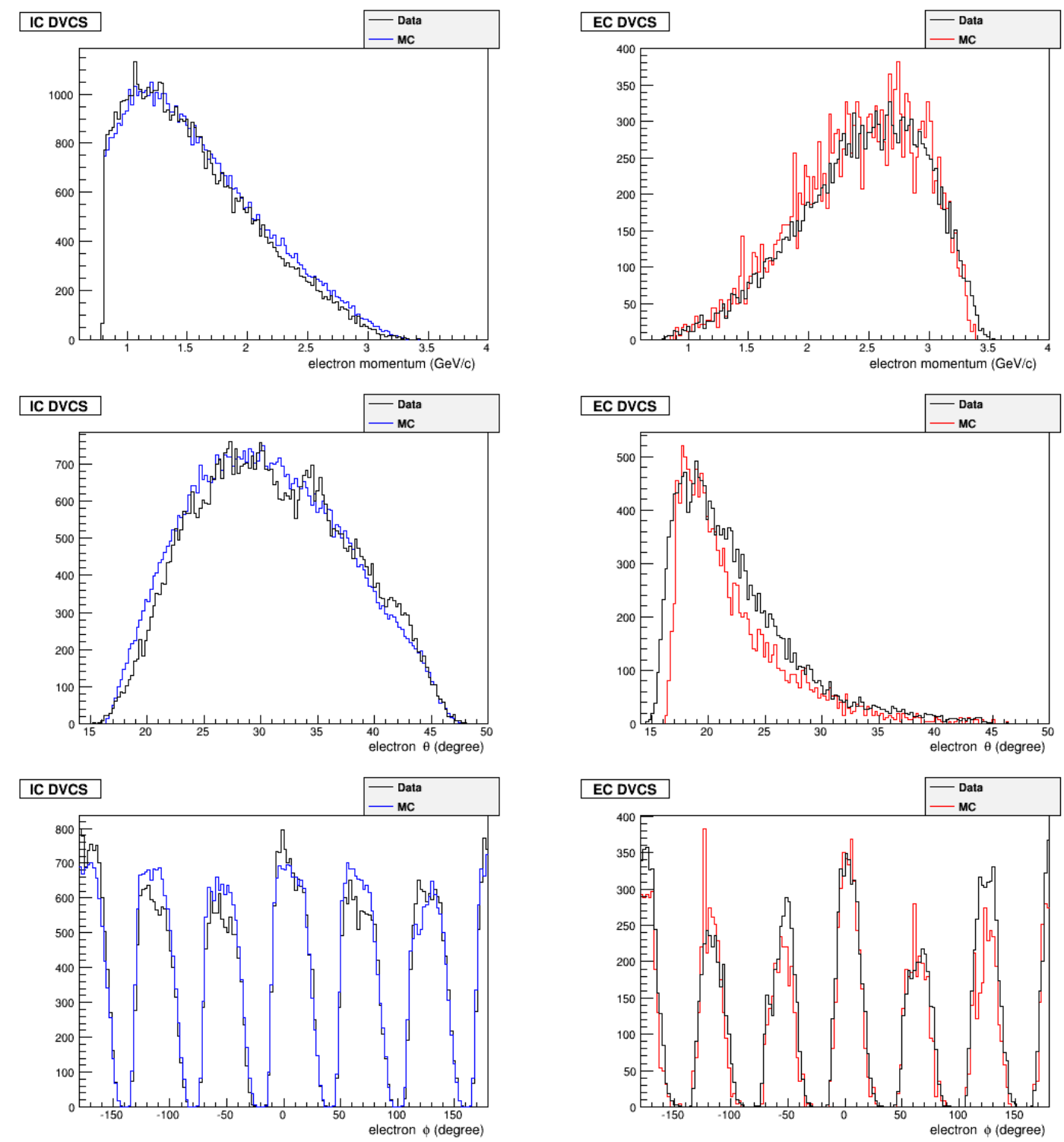

Fig. 3.24: DVCS channel. Comparison of data and Monte-Carlo simulation for DVCS events with the photon detected in the IC (left) and EC (right). Electron kinematic variables. From the first row down, the momentum $p$, the polar angle $\theta$, and the azimuthal angle $\phi$ are plotted. 

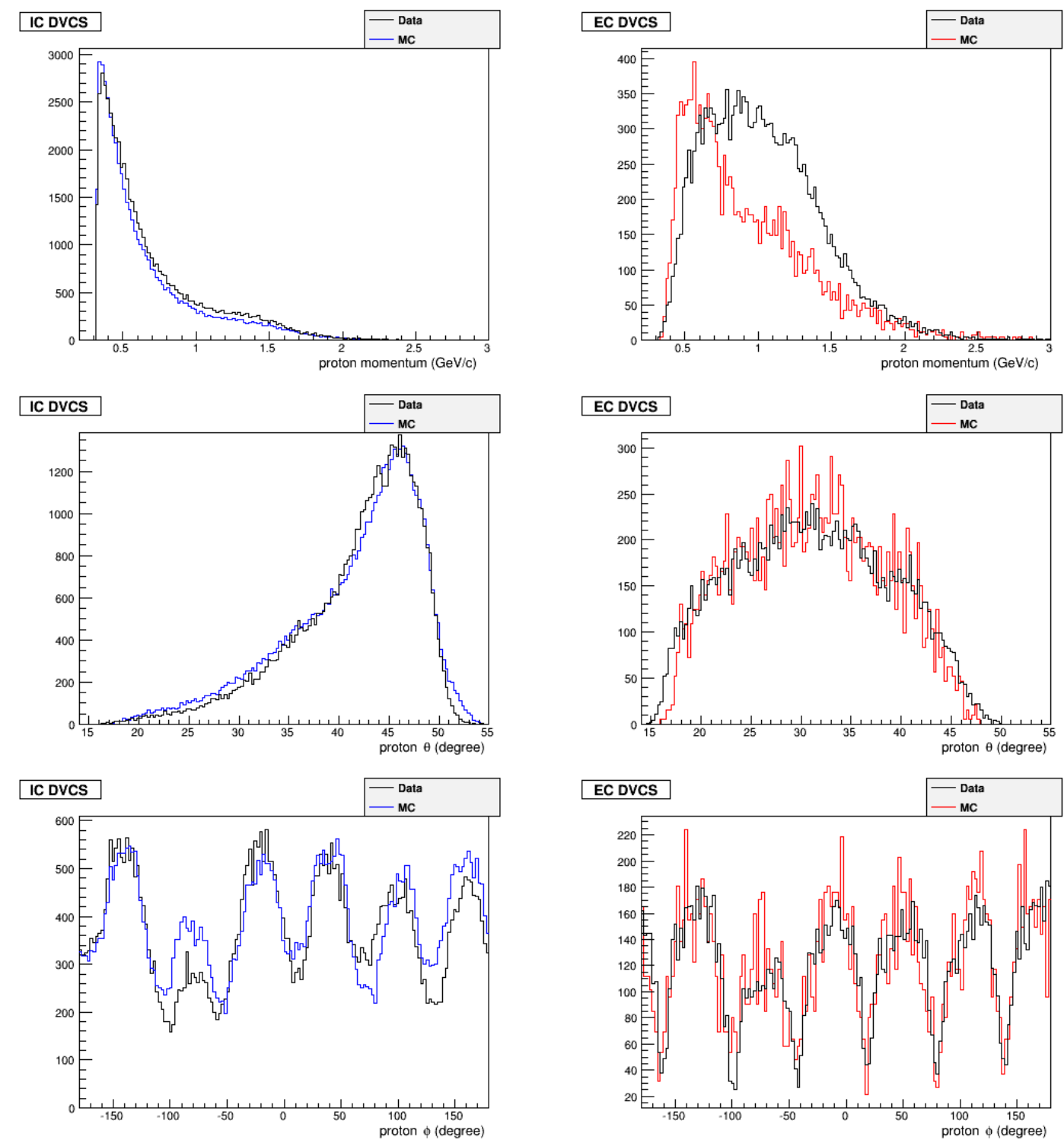

Fig. 3.25: DVCS channel. Comparison of data and Monte-Carlo simulation for DVCS events with the photon detected in the IC (left) and EC (right). Proton kinematic variables. From the first row down, the momentum $p$, the polar angle $\theta$, and the azimuthal angle $\phi$ are plotted. 

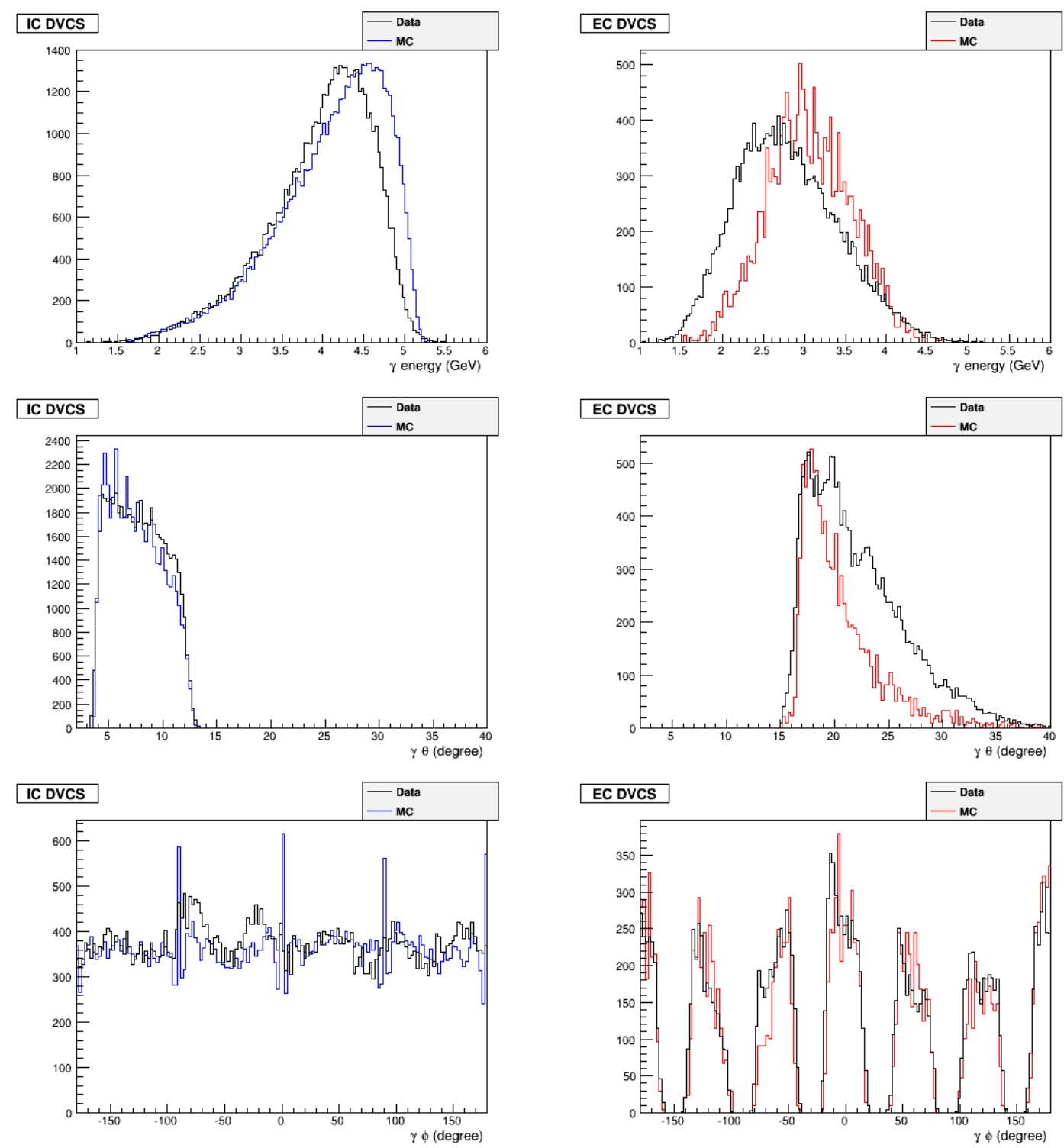

Fig. 3.26: DVCS channel. Comparison of data and Monte-Carlo simulation for DVCS events with the photon detected in the IC (left) and EC (right). Photon kinematic variables. From the first row down, the momentum $p$, the polar angle $\theta$, and the azimuthal angle $\phi$ are plotted. 


\subsubsection{Exclusive $\pi^{0}$ simulation}

Exclusive $e p \pi^{0}$ events were generated using the "aoo_rad" code [1], which had been modified including a parametrization of the $e p \pi^{0}$ differential cross sections that have recently been measured by CLAS [43]. Figures 3.27 - 3.30 show comparisons of the distributions of kinematic variables for the data (left column) and the Monte-Carlo simulation (right column)

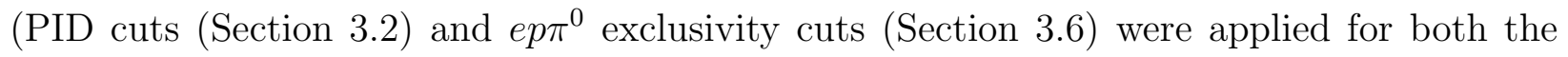
data and the Monte-Carlo). 

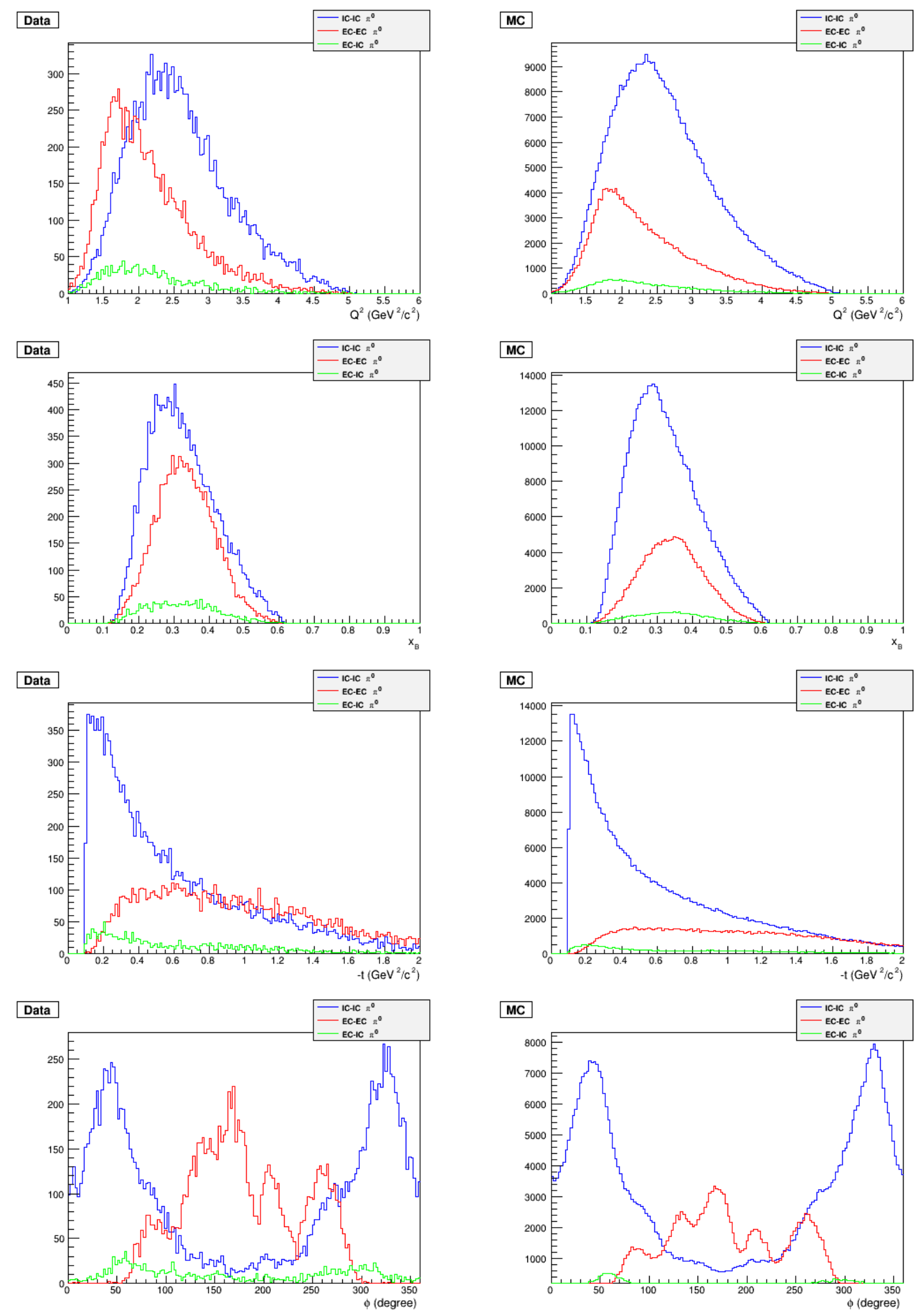

Fig. 3.27: Exclusive $\pi^{0}$ channel. Comparison of data (left column) and Monte-Carlo simulation (right column). From the first row down, $Q^{2}, x_{B},-t$ and $\phi$ are plotted. The blue, red, and green curves correspond to the three photon-detection topologies: IC-IC (blue), EC-EC (red), EC-IC (green). 

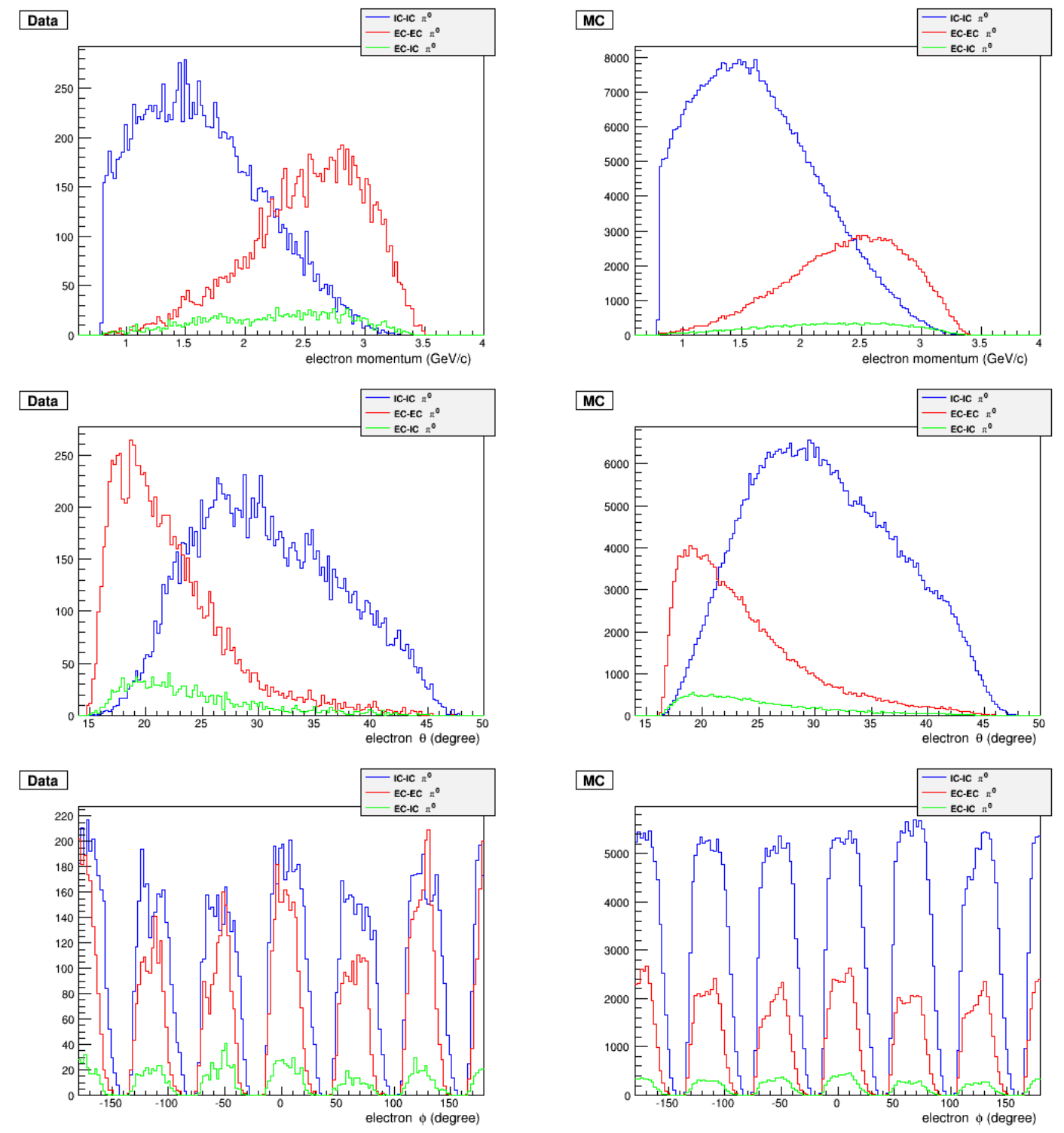

Fig. 3.28: Exclusive $\pi^{0}$ channel. Comparison of data (left column) and Monte-Carlo simulation (right column). Electron kinematic variables. From the first row down, the momentum $p$, the polar angle $\theta$, and the azimuthal angle $\phi$ are plotted. The blue, red, and green curves correspond to the three photon-detection topologies: IC-IC (blue), EC-EC (red), EC-IC (green). 

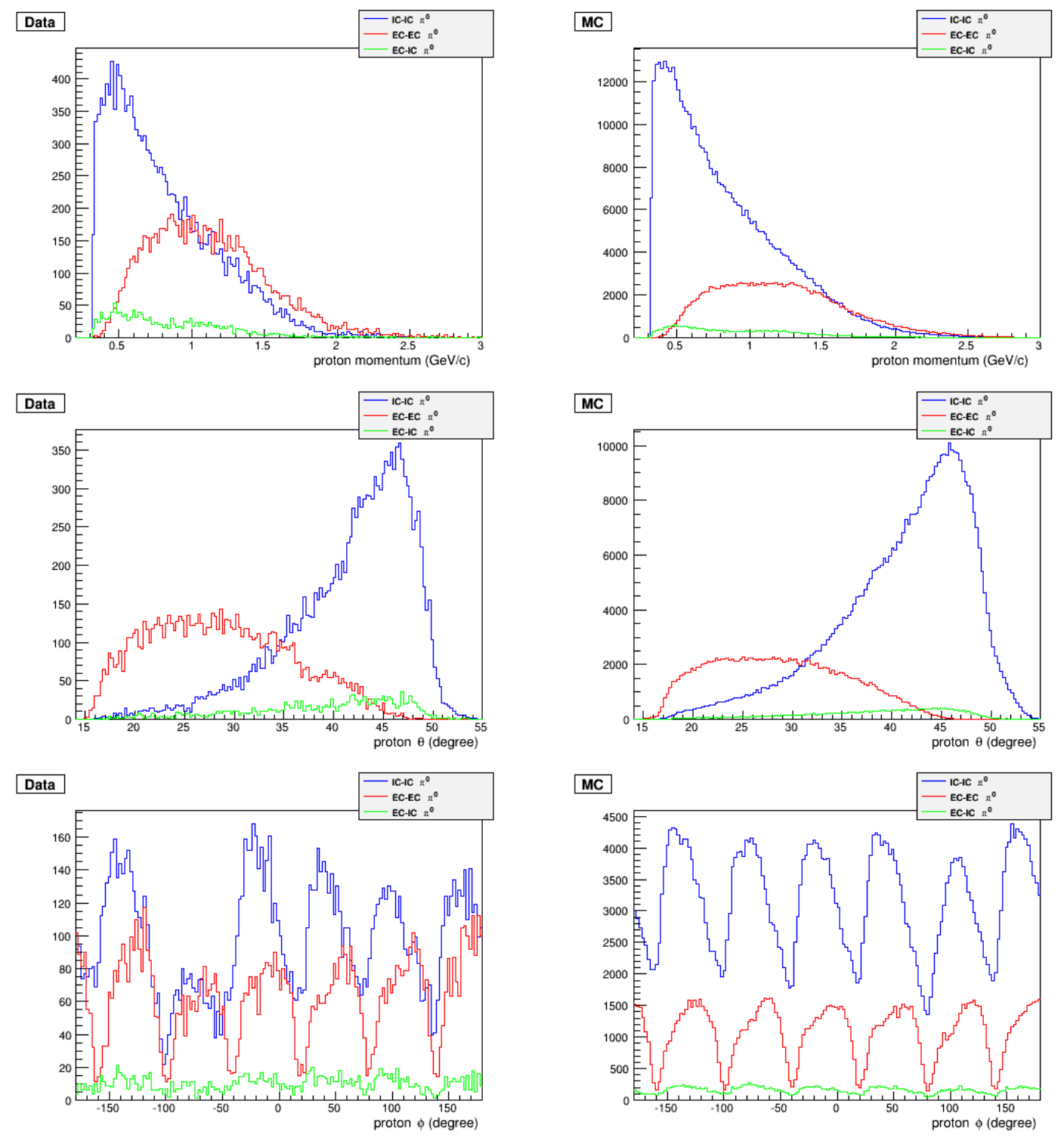

Fig. 3.29: Exclusive $\pi^{0}$ channel. Comparison of data (left column) and Monte-Carlo simulation (right column). Proton kinematic variables. From the first row down, the momentum $p$, the polar angle $\theta$, and the azimuthal angle $\phi$ are plotted. The blue, red, and green curves correspond to the three photon-detection topologies: IC-IC (blue), EC-EC (red), EC-IC (green). 

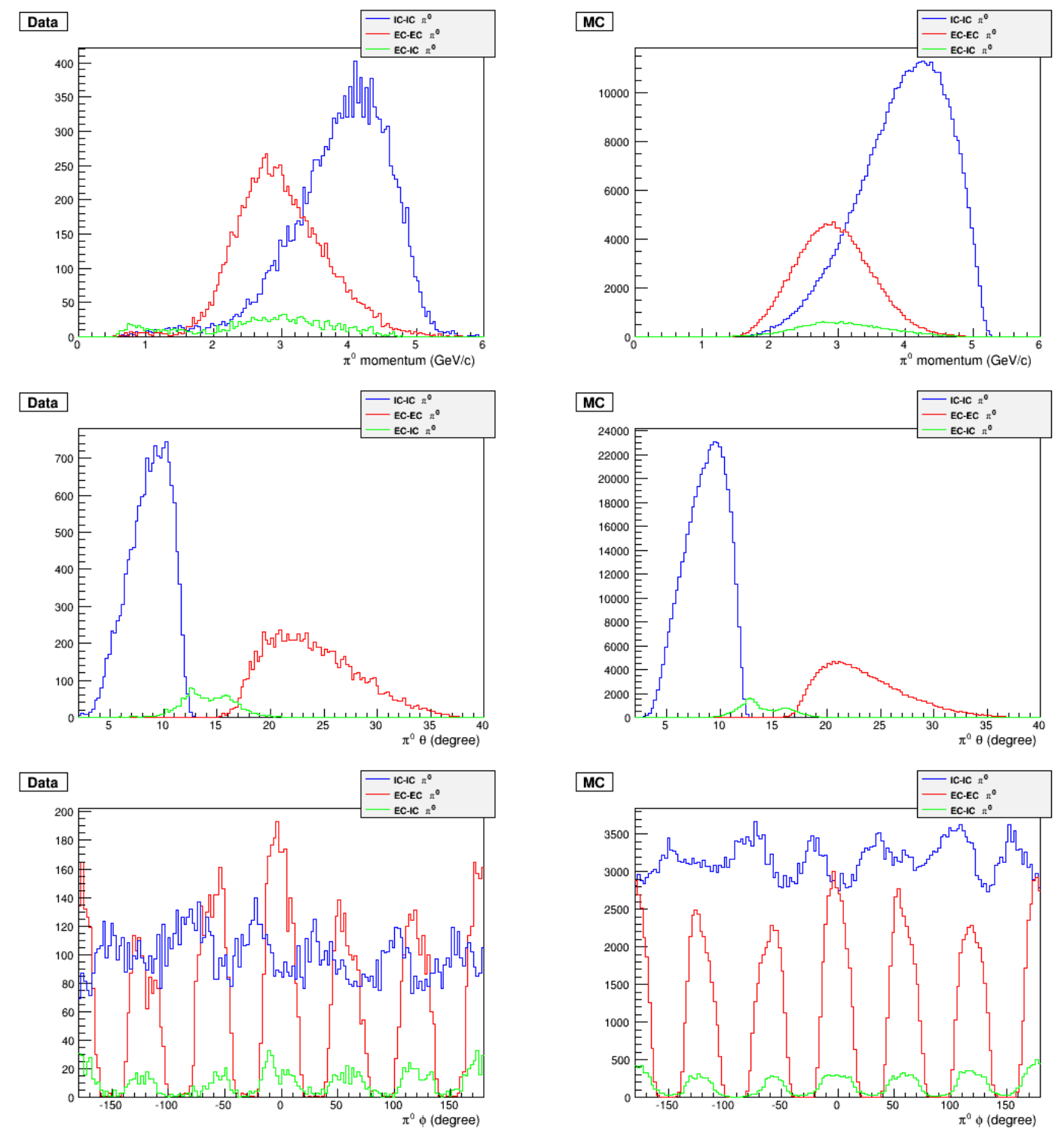

Fig. 3.30: Exclusive $\pi^{0}$ channel. Comparison of data (left column) and Monte-Carlo simulation (right column). $\pi^{0}$ kinematic variables. From the first row down, the momentum $p$, the polar angle $\theta$, and the azimuthal angle $\phi$ are plotted. The blue, red, and green curves correspond to the three photon-detection topologies: IC-IC (blue), EC-EC (red), EC-IC (green). 


\subsection{DVCS Channel Selection}

After selecting events with exactly one electron and one proton, and at least one photon, and having applied the momentum and angle corrections described in Sections 4.5 and 4.4, further cuts need to be applied to ensure the exclusivity of the DVCS/Bethe-Heitler final state. There are two main sources of background in the data: the nuclear background coming from scattering on the nitrogen of the $\mathrm{NH}_{3}$ target as well as the background coming from other channels containing electron, proton and at least one photon in the final state (mainly the exclusive $\pi^{0}$ channel). Cuts on the following observables, or "exclusivity variables", were chosen to reduce the background and ensure the selection of the DVCS/Bethe-Heitler events:

- $M M^{2}(e p)$, the squared missing mass of the ep system,

- $\theta_{\gamma X}$, the angle between the measured photon and the calculated photon, using the detected electron and proton,

- $\Delta \phi$, the difference between two ways to compute the $\phi$ angle (the angle between the leptonic and the hadronic planes). The two ways concern the definition of the normal vector to the hadronic plane: one is via the cross product of proton and real photon, and the other one is via the cross product of the proton and the virtual photon

- $p_{\text {perp }}$, the transverse component of the missing momentum of the reaction $e p \rightarrow e p \gamma X$, $p_{\text {perp }}=\sqrt{p_{x}(X)^{2}+p_{y}(X)^{2}}$

It was important to be sure that the exclusivity cuts were determined in a consistent

way for both the data and the Monte-Carlo simulation used to compute the $\pi^{0}$ background contamination to the DVCS-Bethe-Heitler events (Section 4.7). As is discussed in Section 3.4 , the simulation was done as close as possible to the experimental data, however, the particle resolutions are not perfectly reproduced. A method of selection was developed so that the same fraction of events for both data and simulation would be kept by fitting each exclusivity variable separately for data and simulation with a Gaussian and cutting $\pm 3 \sigma$ around the fitted mean. The fits were done on the distributions after subtracting the carbon 
data scaled by the ratio of $\mathrm{NH}_{3}$ to carbon Faraday-cup counts from the $\mathrm{NH}_{3}$ data in order to eliminate possible smearing effects stemming from nuclear background.

The exclusivity variables to be fit were plotted after applying PID cuts (Section 3.2), momentum and angle corrections, as well as the preliminary set of cuts of:

- "DIS cuts", to be away from the resonance region and ensure the applicability of the GPD formalism: $Q^{2}>1 \mathrm{GeV}^{2}, W>2 \mathrm{GeV}^{2}, Q^{2}>-t$

- $E_{\gamma}>1 \mathrm{GeV}$, as the DVCS and Bethe-Heitler photons are expected to have high energy

- $3 \sigma$ cut around the mean of $M M^{2}(e p)$ to eliminate, from the experimental data, the background from other channels than DVCS-Bethe-Heitler or ep $\pi^{0}$ (visible, for instance, in Figure 3.31, where peaks from $\eta$ and $\omega / \rho$ are evident.

The distributions were fit after applying only the preliminary cuts listed above (and not the other exclusivity cuts) in order to avoid distortion to the distributions coming from cutting on correlated variables. The reliability of this method regarding this aspect was checked by comparing the widths of the peaks on the exclusivity variables before and after the final cuts were applied.

As the DVCS photon was detected by either the Inner calorimeter (IC) or CLAS's Electromagnetic Calorimeter (EC), and the two calorimeters have different resolutions, the two photon-detection typologies were considered separately. The next two sections detail the cut selection for each topology.

\subsubsection{IC Photon}

Figures 3.31 - 3.34 show the results of the exclusivity-cuts analysis performed on the ICtopology data for Run Period B (plots for Run Period A are shown in Appendix A) with a summary of cuts listed in Table 3.5 for data and Table 3.6 for MC. The cuts, shown as green lines, were determined as described in the previous section: Gaussian fits done separately to data and MC, and a $3 \sigma$ cut placed around the fitted means. For some of the distributions, that are not exactly gaussian, it was still decided to perform a gaussian fit, using a restricted fitting range. The stability of the obtained widths and mean was checked by comparing 
the results obtained by two independent data analyses on the Run Periods A and B of the EG1-DVCS experiment. Each plot shows the data on the two top plots and the Monte-Carlo simulation for DVCS/Bethe-Heitler in the two bottom plots. The plots on the left are before all cuts but the preliminary ones, the ones on the right are after all cuts but the one on the variable plotted. The fairly good agreement, after application of the cut on $M M^{2}(e p)$, of data and simulation with respect to shapes of distributions, peak positions and widths, hints that this topology is strongly dominated by the DVCS channel, with a minor contribution from pi0 contamination.

\begin{tabular}{|l|c|c|}
$\begin{array}{c}\text { Exclusivity } \\
\text { Variable }\end{array}$ & $\begin{array}{c}\text { Run Period A } \\
\text { Exclusivity Cut }\end{array}$ & $\begin{array}{c}\text { Run Period B } \\
\text { Exclusivity Cut }\end{array}$ \\
\hline \hline$M M^{2}(e p)$ & $>-0.167$ & $>-0.150$ \\
& $<0.181$ & $<0.161$ \\
\hline$\theta_{\gamma X}$ & $<1.055$ & $<0.976$ \\
\hline$\Delta \phi$ & $>-1.773$ & $>-1.495$ \\
& $<1.647$ & $<1.289$ \\
\hline$p_{\text {perp }}$ & $<0.096$ & $<0.095$ \\
\hline
\end{tabular}

Table 3.5: DVCS selection, IC topology. Summary of DVCS exclusivity cuts on data. Independent data analyses were performed on the Run Periods A and B of the EG1-DVCS experiment. 
Exclusivity Run Period A Run Period B

Variable Exclusivity Cut Exclusivity Cut

\begin{tabular}{|l|c|c|}
\hline \hline$M M^{2}(e p)$ & $>-0.128$ & $>-0.134$ \\
& $<0.218$ & $<0.138$ \\
\hline$\theta_{\gamma X}$ & $<1.118$ & $<1.041$ \\
\hline$\Delta \phi$ & $>-1.899$ & $>-1.694$ \\
& $<1.737$ & $<1.546$ \\
\hline$p_{\text {perp }}$ & $<0.084$ & $<0.083$ \\
\hline
\end{tabular}

Table 3.6: DVCS selection, IC topology. Summary of DVCS exclusivity cuts on MC. Independent data analyses were performed on the Run Periods A and B of the EG1-DVCS experiment. 

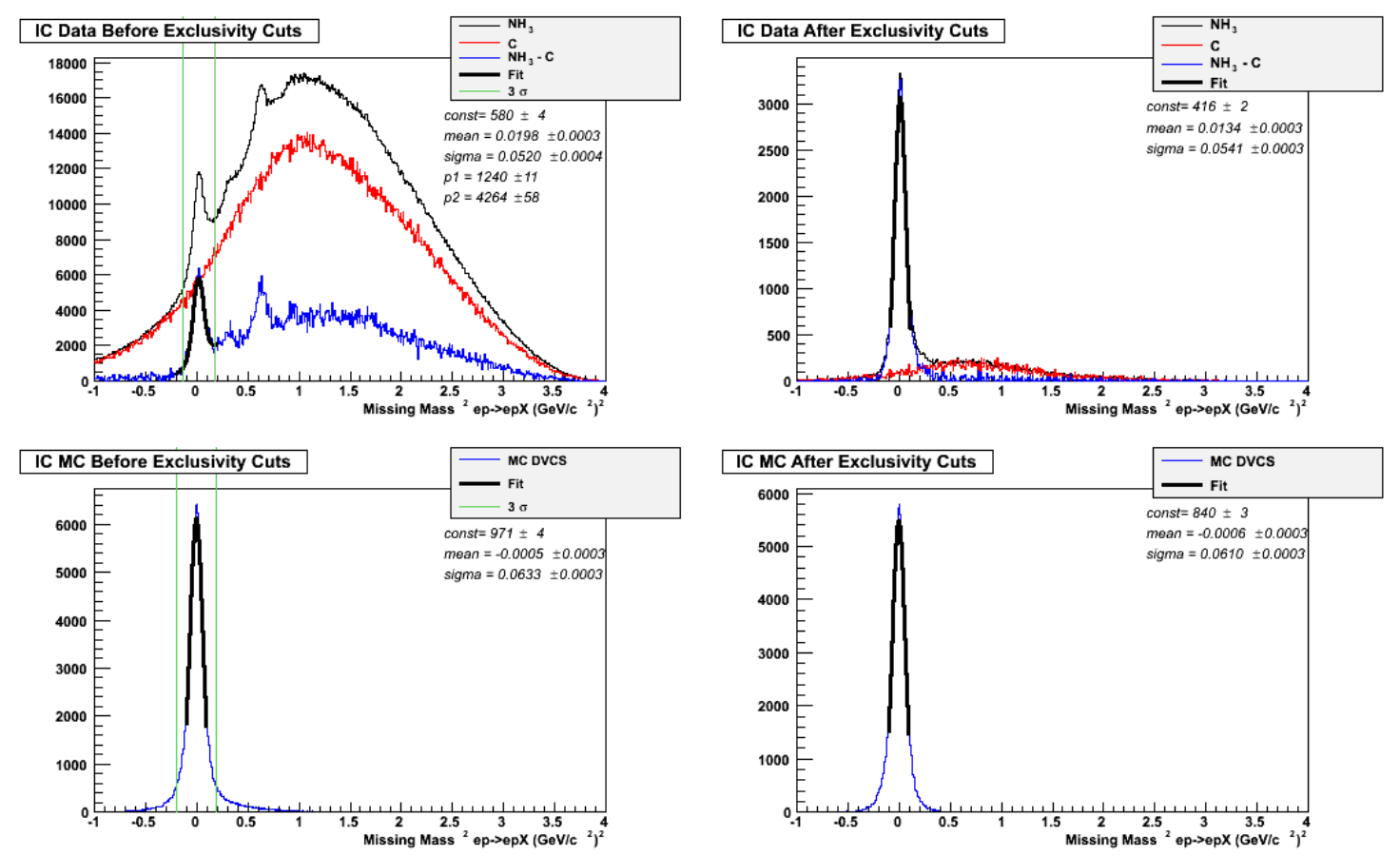

Fig. 3.31: DVCS selection Run Period B, IC topology Missing mass of the ep system. Top left: without any but the preliminary "DIS" and $E_{\gamma}$ cuts applied for $\mathrm{NH}_{3}$ data (black), carbon data (red), $\mathrm{NH}_{3}$ data after carbon subtraction (blue); the thick black line is a Gaussian-plus-linear-background fit to the blue curve; the $\pm 3 \sigma$-cut window is indicated by the two green vertical lines. Top right, after all exclusivity cuts, except the one on $M M^{2}(e p)$, have been applied, same color code as the top-left plot. Bottom left, DVCS/Bethe-Heitler Monte-Carlo simulation, without any but the preliminary "DIS" and $E_{\gamma}$ cuts, fitted with a Gaussian curve (black thick line). Bottom right: DVCS/Bethe-Heitler Monte-Carlo simulation, after all exclusivity cuts except the one on $M M^{2}(\mathrm{ep})$ have been applied, fitted with a Gaussian curve (black thick line). 

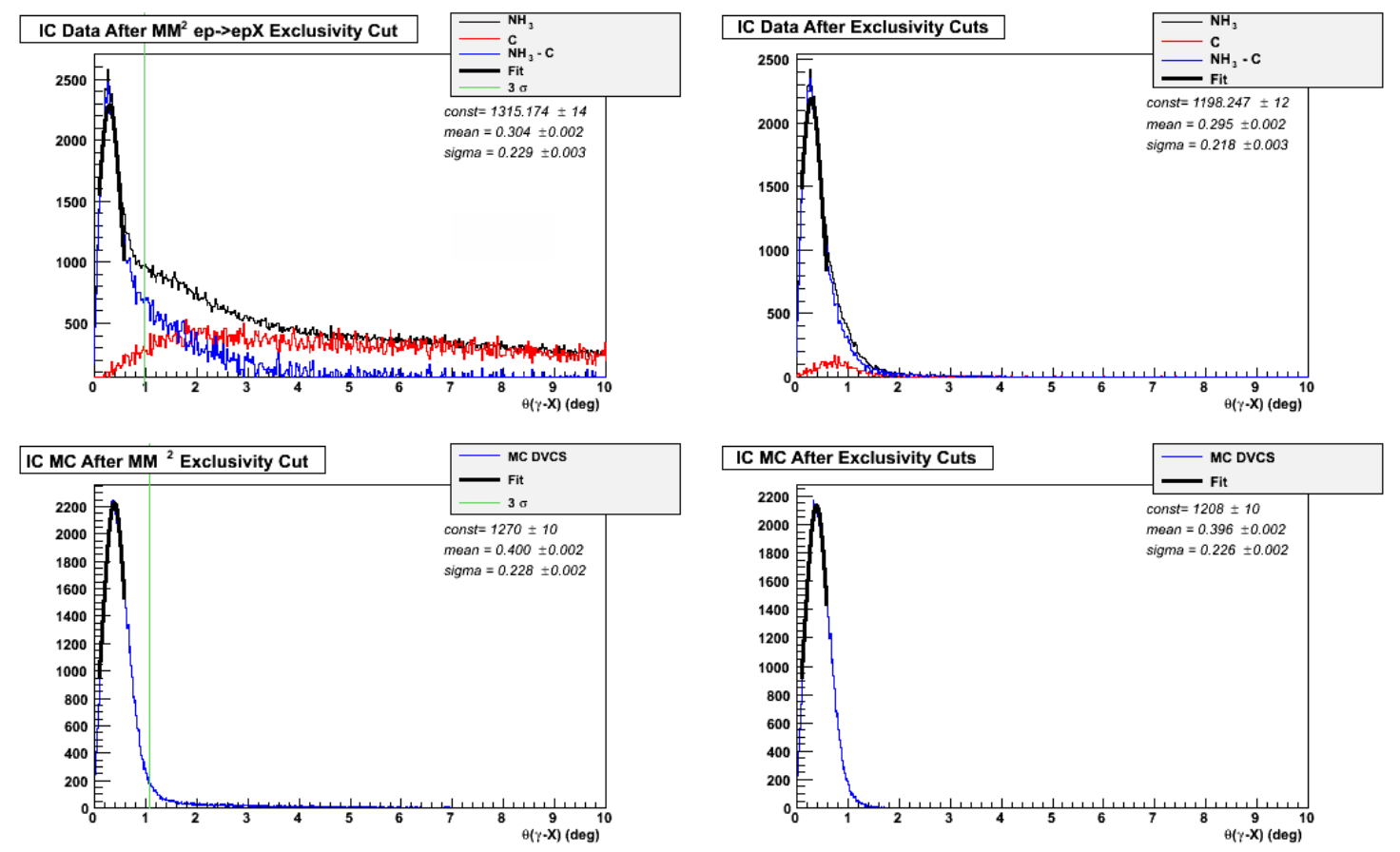

Fig. 3.32: DVCS selection Run Period B, IC topology $\theta_{\gamma X}$ distributions. Top left, with "DIS" and $E_{\gamma}$ cuts plus a $3 \sigma$ cut on $M M^{2}(e p)$ applied for $N H_{3}$ data (black), carbon data (red), $\mathrm{NH}_{3}$ data after carbon subtraction (blue); the thick black line is a Gaussian fit to the blue curve, the $\pm 3 \sigma$-cut window is indicated by the two green vertical lines. Top right, after all exclusivity cuts, except the one on $\theta_{\gamma X}$, have been applied, same color code as the top-left plot. Bottom left, DVCS/Bethe-Heitler Monte-Carlo simulation, without any but the preliminary "DIS" and $E_{\gamma}$ cuts plus a $3 \sigma$ cut on $M M^{2}(e p)$, fitted with a Gaussian curve (black thick line). Bottom right: DVCS/Bethe-Heitler Monte-Carlo simulation, after all exclusivity cuts except the one on $\theta_{\gamma X}$ have been applied, fitted with a Gaussian curve (black thick line). 

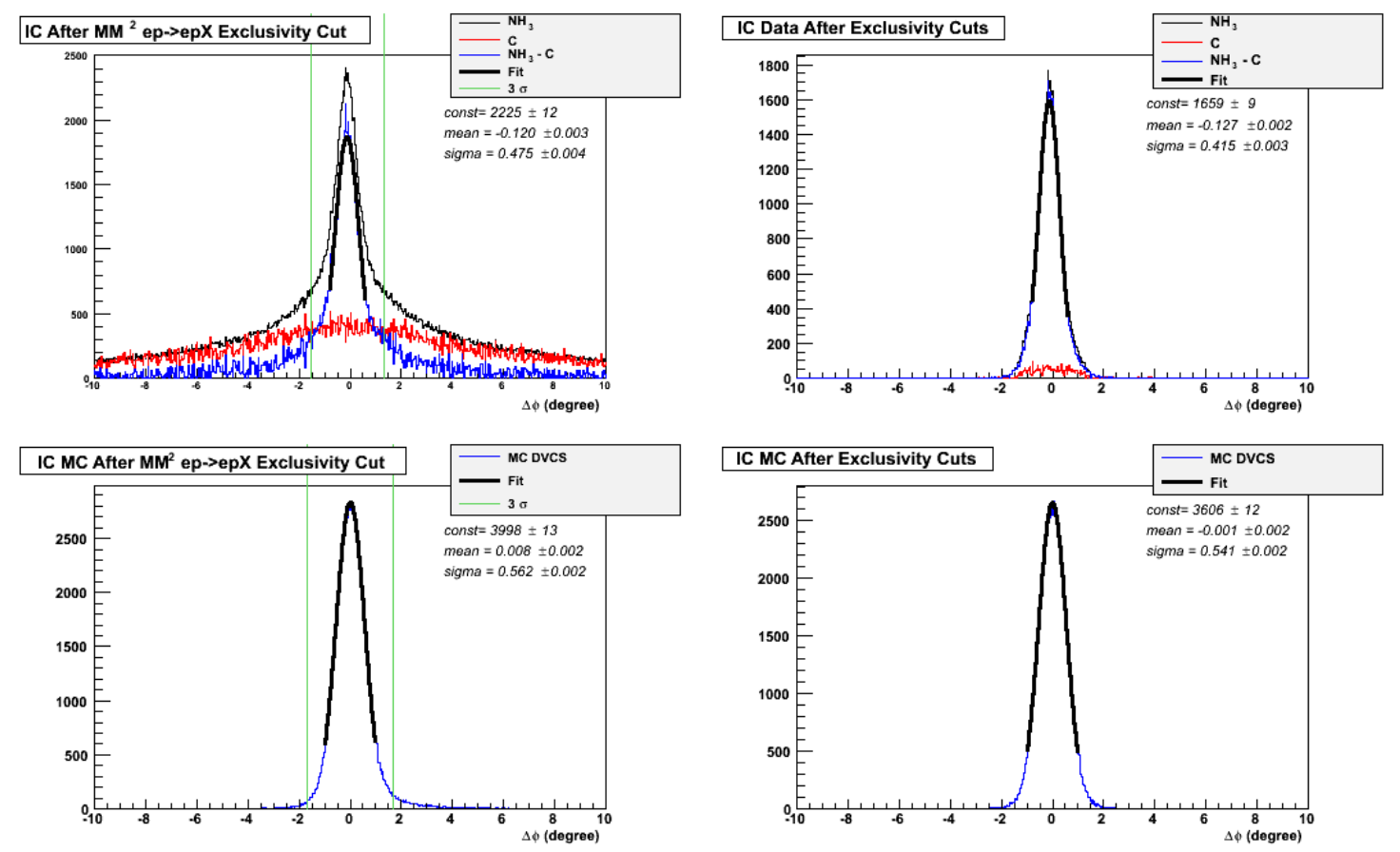

Fig. 3.33: DVCS selection Run Period B, IC topology $\Delta \phi$ distributions. Top left, with "DIS" and $E_{\gamma}$ cuts plus a $3 \sigma$ cut on $M M^{2}(e p)$ applied for $N H_{3}$ data (black), carbon data (red), $\mathrm{NH}_{3}$ data after carbon subtraction (blue); the thick black line is a Gaussian fit to the blue curve, the $\pm 3 \sigma$-cut window is indicated by the two green vertical lines. Top right, after all exclusivity cuts except the one on $\Delta \phi$ have been applied, same color code as the top-left plot. Bottom left, DVCS/Bethe-Heitler Monte-Carlo simulation, without any but the preliminary "DIS" and $E_{\gamma}$ cuts plus a $3 \sigma$ cut on $M M^{2}(e p)$, fitted with a Gaussian curve (black thick line). Bottom right: DVCS/Bethe-Heitler Monte-Carlo simulation, after all exclusivity cuts except the one on $\Delta \phi$ have been applied, fitted with a Gaussian curve (black thick line). 

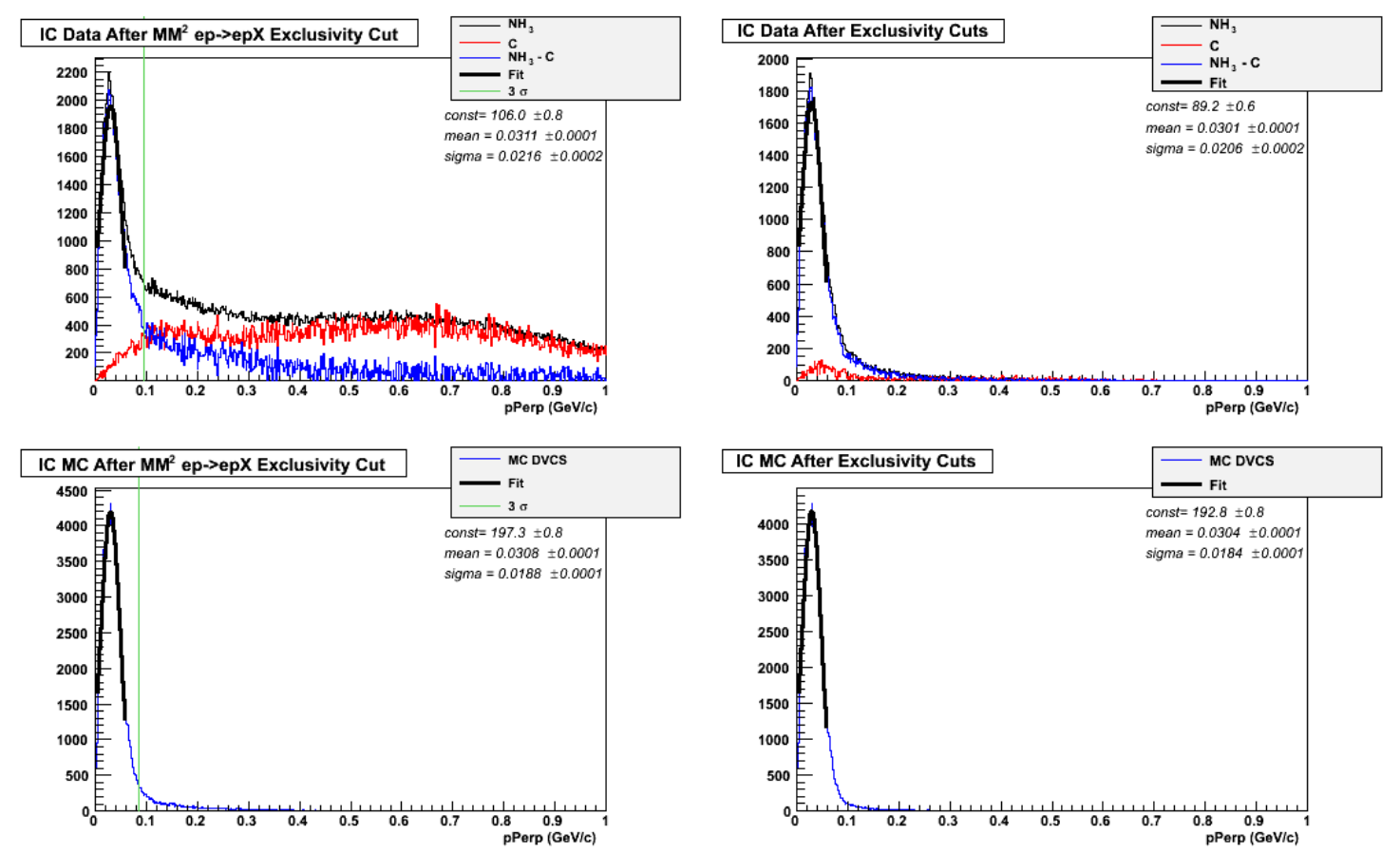

Fig. 3.34: DVCS selection Run Period B, IC topology $p_{\text {perp }}$ distributions. Top left, with "DIS" and $E_{\gamma}$ cuts plus a $3 \sigma$ cut on $M M^{2}(e p)$ applied for $N H_{3}$ data (black), carbon data (red), $\mathrm{NH}_{3}$ data after carbon subtraction (blue); the thick black line is a Gaussian fit to the blue curve, the $\pm 3 \sigma$ cut window is indicated by the two green vertical lines. Top right, after all exclusivity cuts except the one on $p_{\text {perp }}$ have been applied, same color code as the top-left plot. Bottom left, DVCS/Bethe-Heitler Monte-Carlo simulation, without any but the preliminary "DIS" and $E_{\gamma}$ cuts plus a $3 \sigma$ cut on $M M^{2}(e p)$, fitted with a Gaussian curve (black thick line). Bottom right: DVCS/Bethe-Heitler Monte-Carlo simulation, after all exclusivity cuts except the one on $p_{\text {perp }}$ have been applied, fitted with a Gaussian curve (black thick line). 


\subsubsection{EC Photon}

A different strategy to define the exclusivity cuts was adopted for the EC topology. Unlike the IC topology, the comparison between the data before all cuts but the preliminary ones, and the DVCS/Bethe-Heitler simulation shows striking differences for all the exclusivity variables. Instead, there seems to be a very good resemblance between the distributions of the data and the ones obtained with the $e p \pi^{0}$ simulation as in Figure 3.35. The discrepancies shown in Figure 3.35 between data and DVCS/Bethe-Heitler simulation could be due to a combination of a bad description of the EC calorimeter in GSIM and of the dominance of the $e p \pi^{0}$ events in this topology. The latter can also be expected from the physics itself, as the greatest majority of the DVCS/Bethe-Heitler events have a high-energy forward-emitted photon which would be detected in the IC, while $\pi^{0}$ 's can decay more or less isotropically in both calorimeters.

As the peaks (which, when visible, are very broad) in the exclusivity variables for the data in this case are not necessarily due to the DVCS channel, it was decided not to fit them to extract cuts means and widths, and to fit instead only the peaks of the DVCS/BetheHeitler Monte-Carlo simulations. To correct for the discrepancies in resolutions, mentioned previously, between data and simulation, the widths of the various exclusivity variables obtained from the fits were multiplied by appropriate scaling factors [44]. These factors were obtained from the comparison data/MC for the ep $\pi^{0}$ channel, in the EC-EC topology (both $\pi^{0}$ decay photons detected in the EC). This channel was chosen because its selection is clean and there are therefore no "smearing" effects to the widths of the various distributions coming from background channels (unlike the ep $\gamma$ final state).

The method proceeded in the following way:

- Exclusive $e p \pi^{0}$ events, with the two photons detected in EC, were selected, according to the procedure that is described in Section 3.6.2.

- The $p_{p e r p}, \theta_{\pi^{0}-X}$ and $\Delta \phi$ distributions were plotted (see Figure 3.36), for both data and $\mathrm{MC}$, for the selected exclusive $e p \pi^{0}$ events.

- Each variable, for both data and Monte-Carlo simulation, was fit with a Gaussian (or 

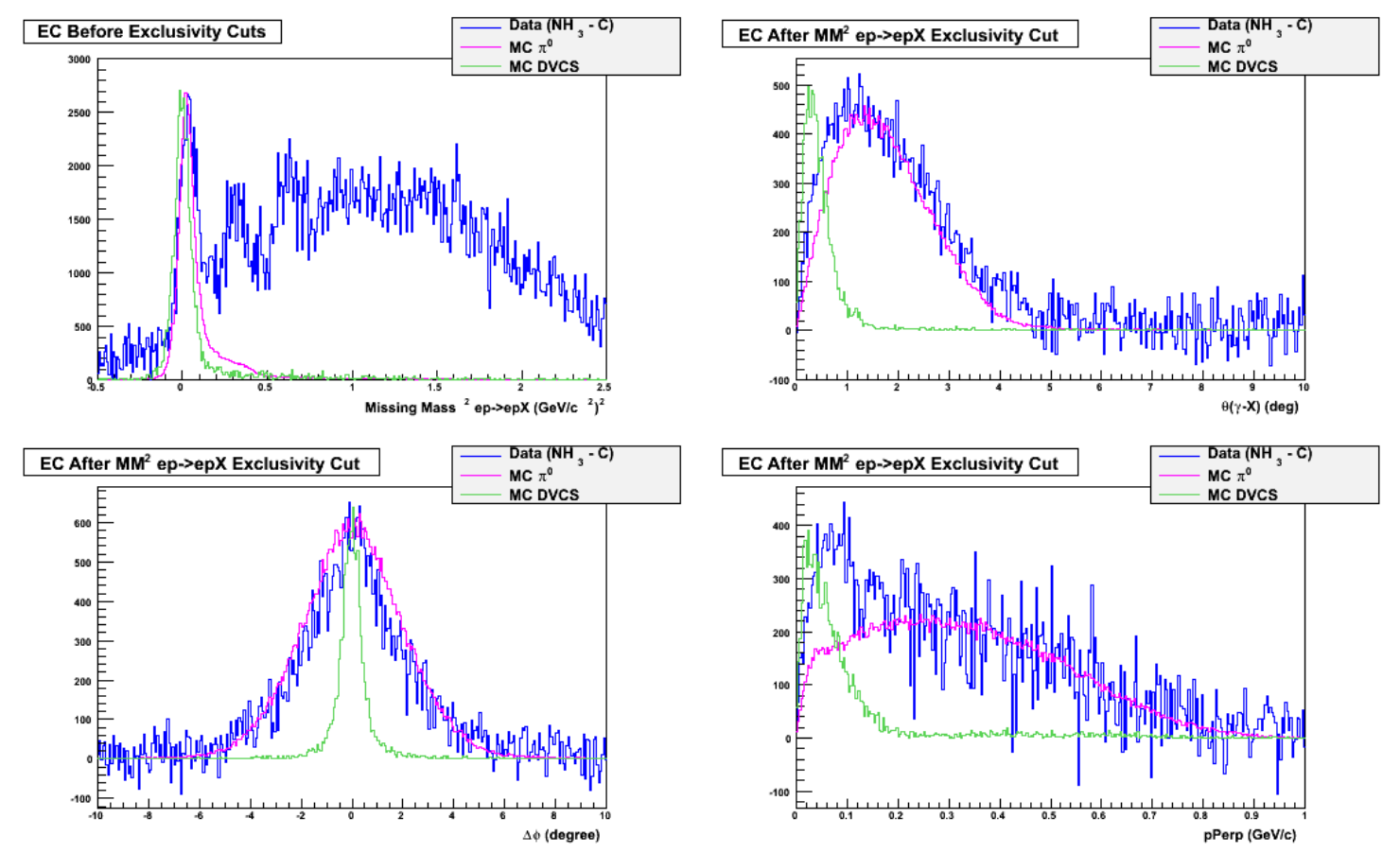

Fig. 3.35: DVCS selection Run Period B, EC topology. Blue represents the $\mathrm{NH}_{3}$ data after carbon subtraction, green is the DVCS/Bethe-Heitler Monte-Carlo simulation, purple is the $e p \pi^{0}$ Monte-Carlo simulation. Top left: $M M^{2}(e p)$, with only DIS and $E_{\gamma}$ cuts applied. Top right: $\theta_{\gamma X}$, after DIS, $E_{\gamma}$, and $M M^{2}(e p)$ cuts applied. Bottom left: $\Delta \phi$, after DIS, $E_{\gamma}$, and $M M^{2}(e p)$ cuts applied. Bottom right: $p_{\text {perp }}$, after DIS, $E_{\gamma}$, and $M M^{2}(e p)$ cuts applied. 
a half-Gaussian, in case of $\left.p_{\text {perp }}\right)$.

- The ratio $r_{1}=\sigma_{d a t a}\left(e p \pi^{0}\right) / \sigma_{M C}\left(e p \pi^{0}\right)$ was computed for each variable. The values obtained, for part $\mathrm{B}$, are: $r_{1}\left(p_{\text {perp }}\right)=1.873, r_{1}\left(\theta_{\gamma \pi^{0}}\right)=1.6 ; r_{1}(\Delta \phi)=1.55 . \quad r_{1}$ is the factor that corrects for the mismatch in resolution between data and GSIM. The correctness of $r_{1}$ for each variable is shown in Figure 3.37, where the MC distributions, after each being "stretched" by its corresponding $r_{1}$, match the data very well.

- Means $\left(M_{M C}(e p \gamma)\right)$ and widths $\left(\sigma_{M C}(e p \gamma)\right)$ of the cuts were determined for the MonteCarlo ep $\gamma$ events, via Gaussian fits ( $\Delta \phi$, which can be fitted fairly decently)) or by placing the cut at the end of the distribution (for $\theta_{\gamma X}$ and $p_{\text {perp }}$, see, respectively, Figures 3.39 and 3.41)

- The cut means and widths for the ep $\mathrm{EC}$ data are finally defined as follows: $M_{\text {data }}(e p \gamma)=$ $r_{1} \cdot M_{M C}(e p \gamma), \sigma_{d a t a}(e p \gamma)=r_{1} \cdot \sigma_{M C}(e p \gamma)$.

The cuts and their effects are shown in Figures 3.38-3.41 for Run Period B (plots for Run Period A are shown in Appendix A) with a summary of cuts listed in Table 3.8.

\begin{tabular}{|l|c|c|}
$\begin{array}{c}\text { Exclusivity } \\
\text { Variable }\end{array}$ & $\begin{array}{c}\text { Run Period A } \\
\text { Exclusivity Cut }\end{array}$ & $\begin{array}{c}\text { Run Period B } \\
\text { Exclusivity Cut }\end{array}$ \\
\hline \hline$M M^{2}(e p)$ & $>-0.105$ & $>-0.114$ \\
& $<0.164$ & $<0.167$ \\
\hline$\theta_{\gamma X}$ & $<2.52$ & $<2.38$ \\
\hline$\Delta \phi$ & $>-1.686$ & $>-1.875$ \\
& $<1.782$ & $<1.979$ \\
\hline$p_{\text {perp }}$ & $<0.284$ & $<0.279$ \\
\hline
\end{tabular}

Table 3.7: DVCS selection, EC topology. Summary of DVCS exclusivity cuts on data. Independent data analyses were performed on the Run Periods A and B of the EG1-DVCS experiment. 

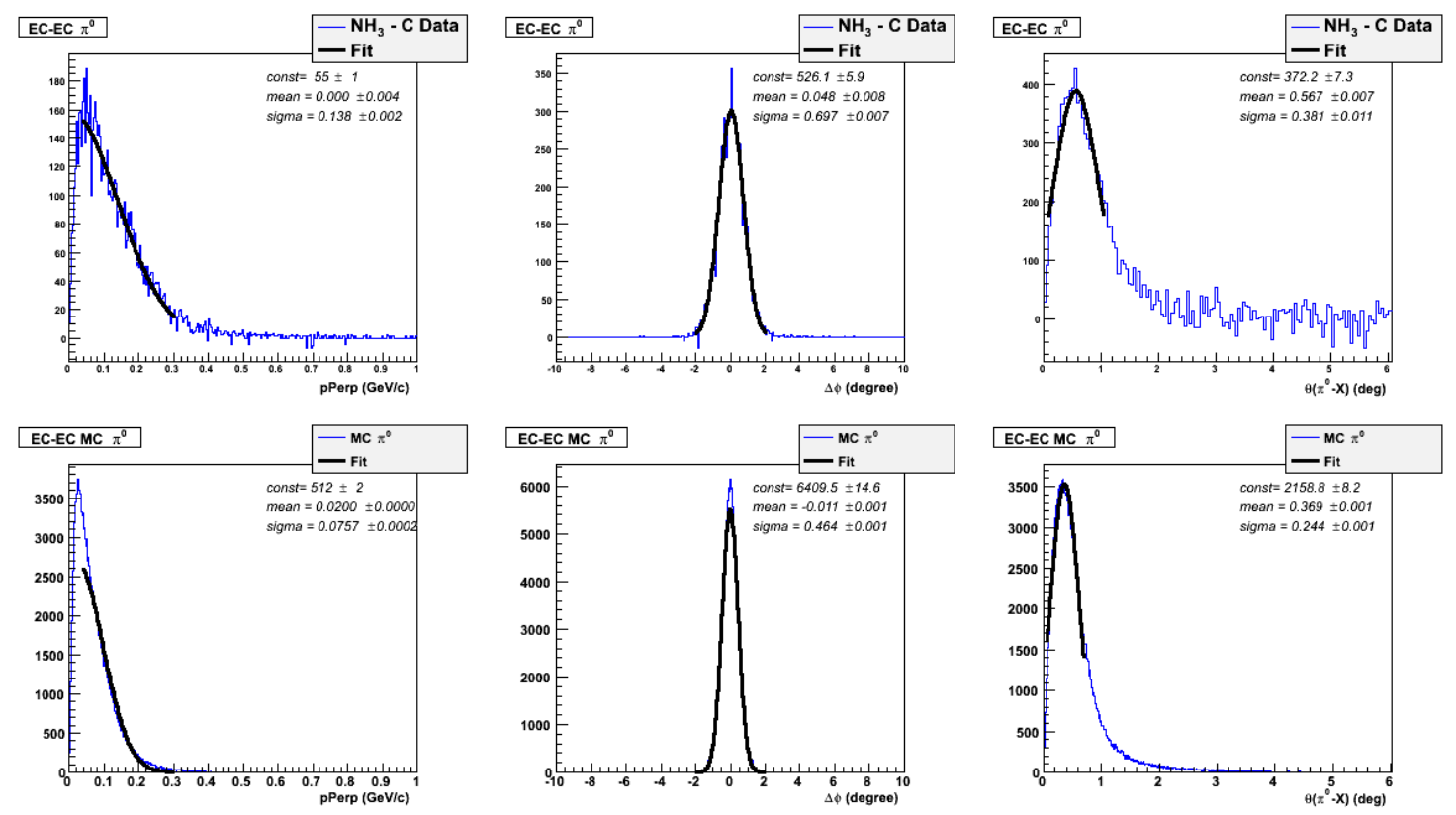

Fig. 3.36: $e p \pi^{0}$ analysis Run Period B, EC-EC topology. Top plots: data, after $e p \pi^{0}$ exclusivity cuts; bottom plots: MC. From left to right: $p_{\text {perp }}$ (fit with a half Gaussian), $\Delta \phi$ and $\theta_{\gamma X}$ (both fit with Gaussians). The ratios of the $\sigma$ 's of the top plots over the ones of the bottom ones gives the factor $r_{1}$.
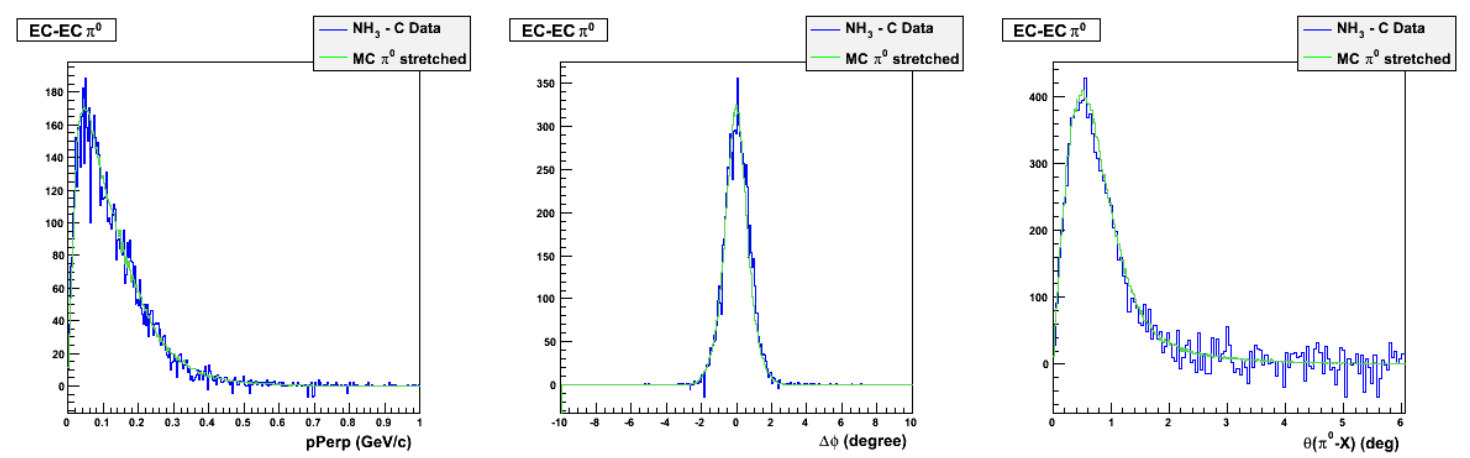

Fig. 3.37: $e p \pi^{0}$ analysis Run Period B, EC-EC topology. From left to right: $p_{\text {perp }}, \Delta \phi$ and $\theta_{\gamma X}$. The data are in blue, the Monte-Carlo, "stretched" by the $r_{1}$ factor, is in green. 

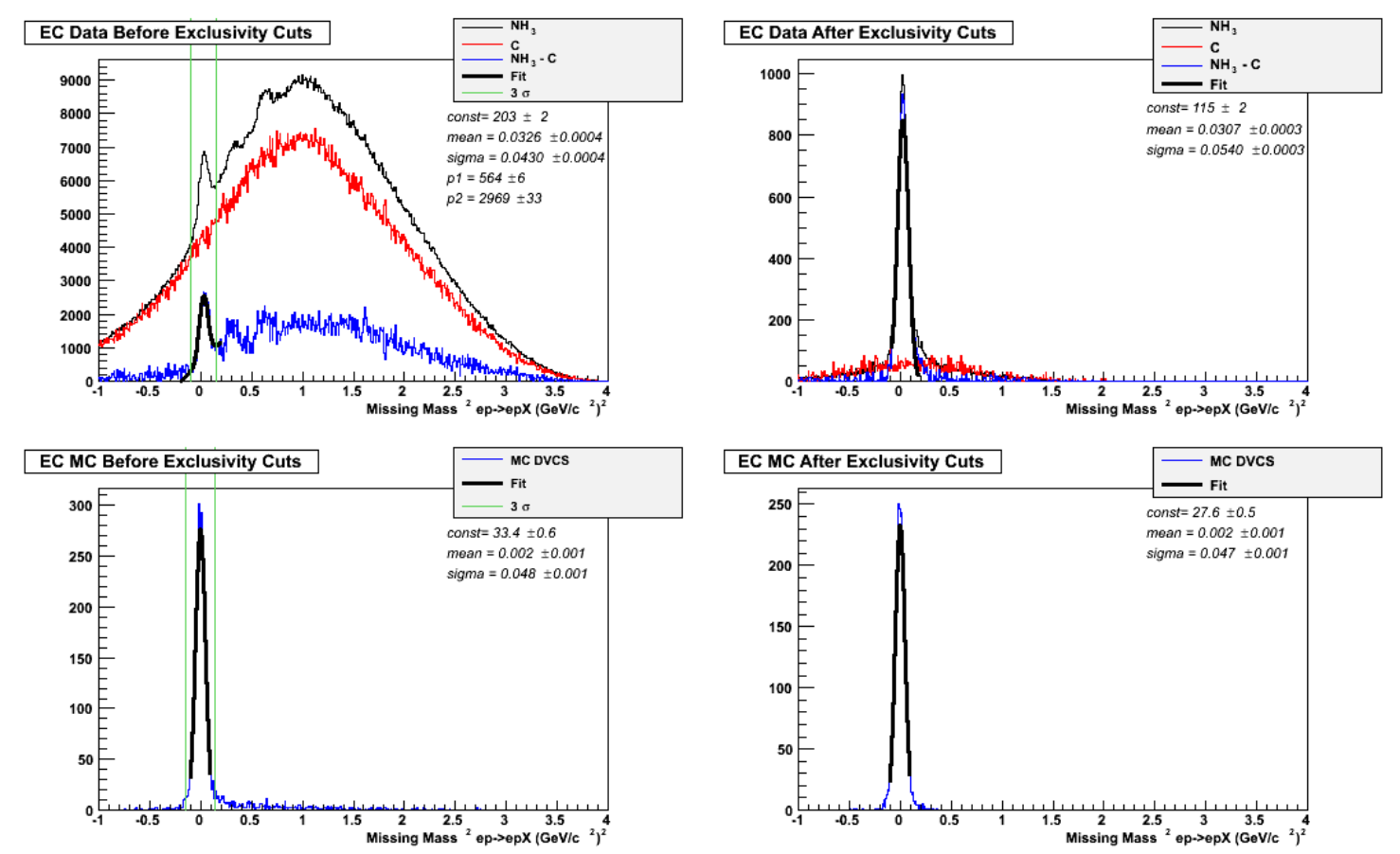

Fig. 3.38: DVCS selection Run Period B, EC topology Missing mass of the ep system. Top left, without any but the preliminary "DIS" and $E_{\gamma}$ cuts applied for $\mathrm{NH}_{3}$ data (black), carbon data (red), $\mathrm{NH}_{3}$ data after carbon subtraction (blue); the thick black line is a Gaussian fit to the blue curve, the $\pm 3 \sigma$-cut window is indicated by the two green vertical lines. Top right, after all exclusivity cuts, except the one on $M M^{2}(e p)$, have been applied, same color code as the top-left plot. Bottom left, DVCS/Bethe-Heitler Monte-Carlo simulation, without any but the preliminary "DIS" and $E_{\gamma}$ cuts, fitted with a Gaussian curve (black thick line). Bottom right: DVCS/Bethe-Heitler Monte-Carlo simulation, after all exclusivity cuts except the one on $M M^{2}(e p)$ have been applied, fitted with a Gaussian curve (black thick line). 

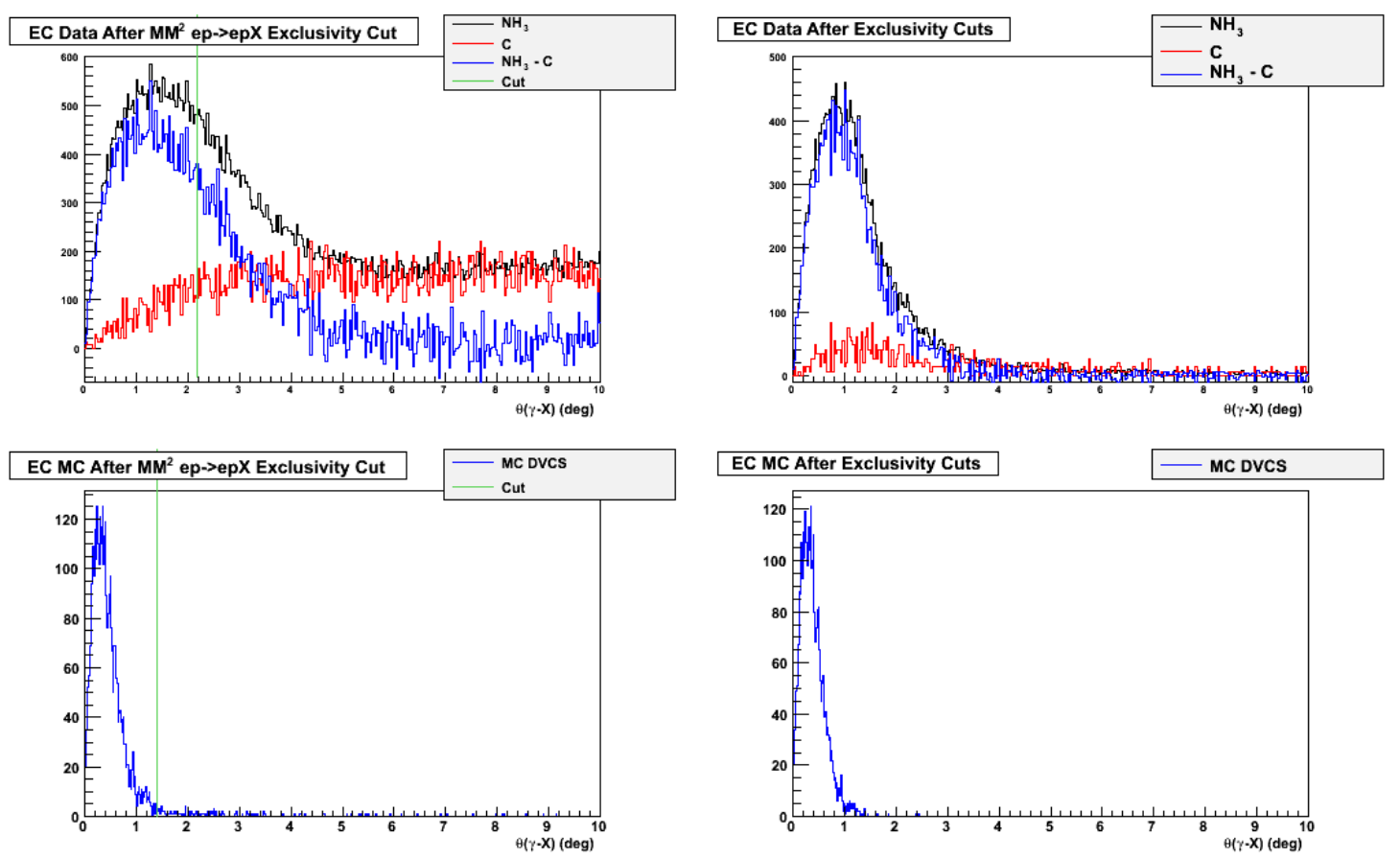

Fig. 3.39: DVCS selection Run Period B, EC topology $\theta_{\gamma X}$ distributions. Top left, with "DIS" and $E_{\gamma}$ cuts plus a $3 \sigma$ cut on $M M^{2}(e p)$ applied for $N H_{3}$ data (black), carbon data (red), $\mathrm{NH}_{3}$ data after carbon subtraction (blue); the green line shows the chosen position of the cut $\left(r_{1} \cdot \sigma_{M C}\right)$. Top right, after all exclusivity cuts, except the one on $\theta_{\gamma X}$, have been applied, same color code as the topleft plot. Bottom left, DVCS/Bethe-Heitler Monte-Carlo simulation, without cuts; the green line shows the chosen position of the cut $\left(\sigma_{M C}\right)$. Bottom right: DVCS/Bethe-Heitler Monte-Carlo simulation, after all exclusivity cuts except the one on $\theta_{\gamma X}$ have been applied. 

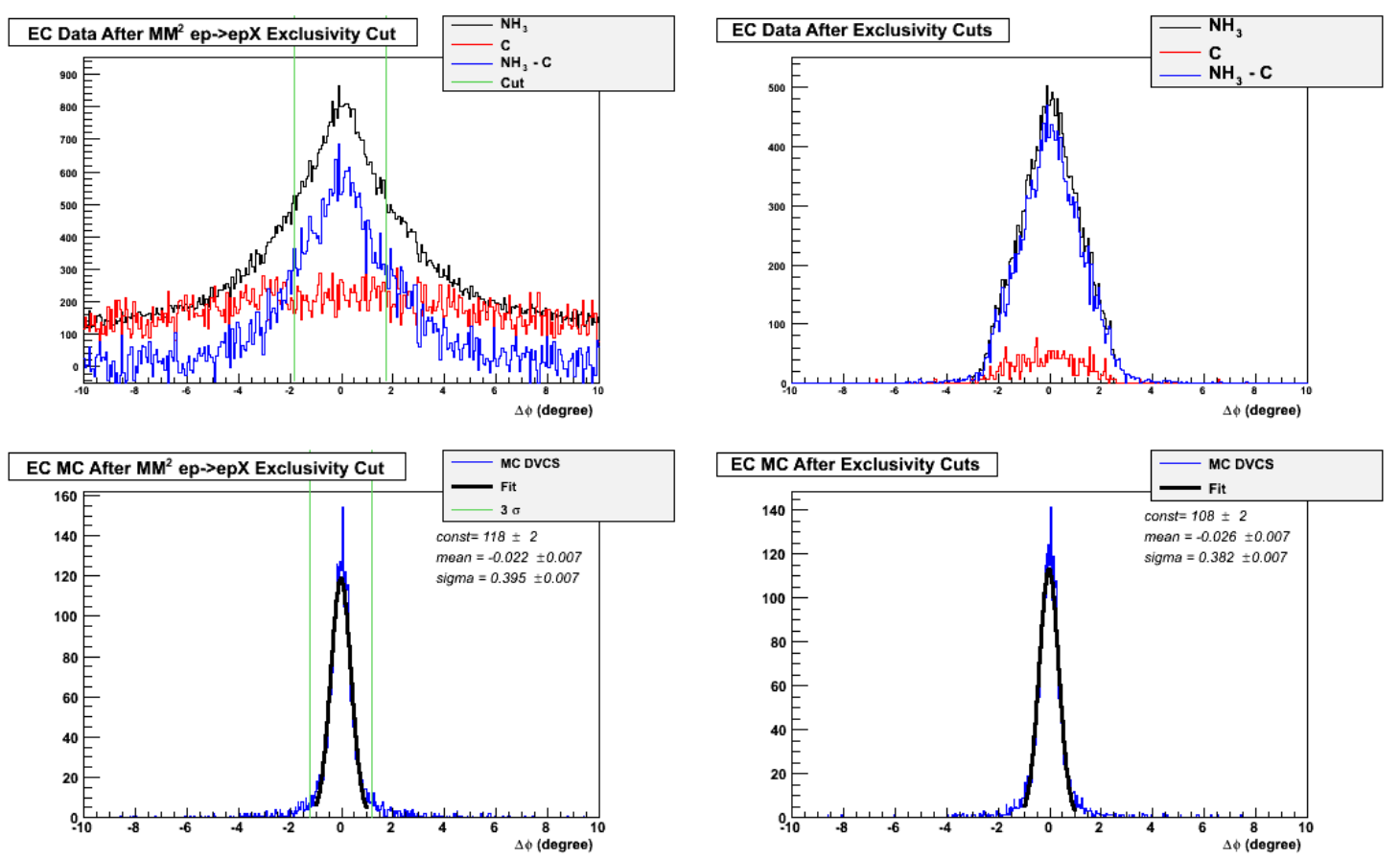

Fig. 3.40: DVCS selection Run Period B, EC topology $\Delta \phi$ distributions. Top left, with "DIS" and $E_{\gamma}$ cuts plus a $3 \sigma$ cut on $M M^{2}(e p)$ applied for $\mathrm{NH}_{3}$ data (black), carbon data (red), $\mathrm{NH}_{3}$ data after carbon subtraction (blue); the green lines show the position of the cut $\left( \pm 3 r_{1} \cdot \sigma_{M C}\right)$. Top right, after all exclusivity cuts except the one on $\Delta \phi$ have been applied, same color code as the top-left plot. Bottom left, DVCS/Bethe-Heitler Monte-Carlo simulation, without cuts, fitted with a Gaussian curve (black thick line); the green lines show the position of the cut $\left( \pm 3 \sigma_{M C}\right)$. Bottom right: DVCS/Bethe-Heitler Monte-Carlo simulation, after all exclusivity cuts except the one on $\Delta \phi$ have been applied, fitted with a Gaussian curve (black thick line). 

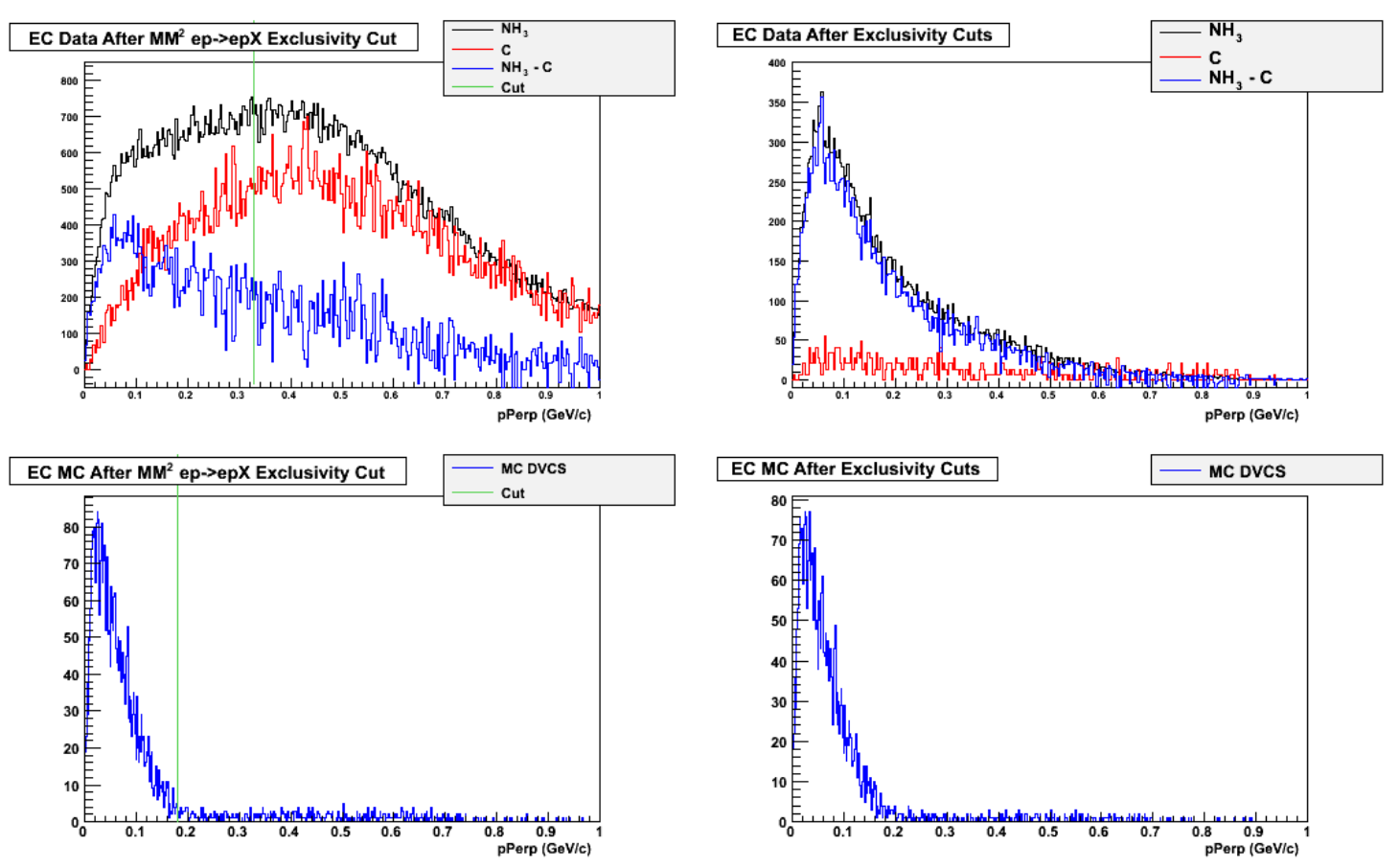

Fig. 3.41: DVCS selection Run Period B, EC topology $p_{\text {perp }}$ distributions. Top left, with "DIS" and $E_{\gamma}$ cuts plus a $3 \sigma$ cut on $M M^{2}(e p)$ applied for $N H_{3}$ data (black), carbon data (red), $\mathrm{NH}_{3}$ data after carbon subtraction (blue)); the green line shows the chosen position of the cut $\left(r_{1} \cdot \sigma_{M C}\right)$. Top right, after all exclusivity cuts except the one on $p_{\text {perp }}$ have been applied. Same color code as the topleft plot. Bottom left, DVCS/Bethe-Heitler Monte-Carlo simulation, without cuts); the green line shows the chosen position of the cut $\left(\sigma_{M C}\right)$. Bottom right: DVCS/Bethe-Heitler Monte-Carlo simulation, after all exclusivity cuts except the one on $p_{\text {perp }}$ have been applied. 
Exclusivity Run Period A Run Period B Variable Exclusivity Cut Exclusivity Cut

\begin{tabular}{|l|c|c|}
\hline \hline$M M^{2}(e p)$ & $>-0.112$ & $>-0.099$ \\
& $<0.116$ & $<0.105$ \\
\hline$\theta_{\gamma X}$ & $<1.40$ & $<1.40$ \\
\hline$\Delta \phi$ & $>-1.094$ & $>-1.205$ \\
& $<1.066$ & $<1.165$ \\
\hline$p_{\text {perp }}$ & $<0.180$ & $<0.180$ \\
\hline
\end{tabular}

Table 3.8: DVCS selection, EC topology. Summary of DVCS exclusivity cuts on MC. Independent data analyses were performed on the Run Periods A and B of the EG1-DVCS experiment. 


\subsection{Exclusive $e p \pi^{0}$ Selection}

Exclusive $\pi^{0}$ electroproduction was used in this analysis to determine the $\pi^{0}$ background contamination to the DVCS-Bethe-Heitler events (Section 4.7). The same method as was used to define the DVCS selection cuts (Section 3.5) was adopted to obtain the exclusivity of the $e p \pi^{0}$ final state. Two additional cuts on IC photons, not applicable to DVCS photons (which have a momentum ¿1 GeV), were applied. They consisted of a low energy threshold of $0.25 \mathrm{GeV}$ and "triangular cut" on $\theta$ versus $E_{\gamma}$ to remove the background coming from Møller electrons. The cut is shown in Figure 3.42 for Run Period B (plots for Run Period A are shown in Appendix B). Three exclusivity variables were used for the selection of the

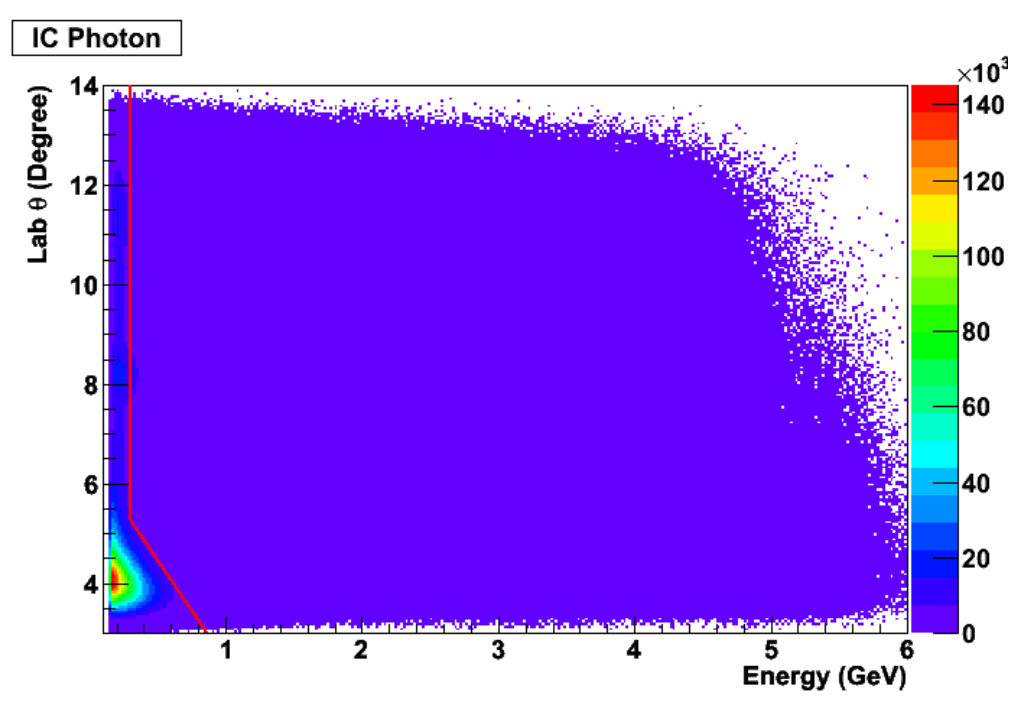

Fig. 3.42: Run Period B: Polar angle $\theta$ as a function of the energy for IC hits, showing the cut on the minimum energy at $0.25 \mathrm{GeV}$, as well as the "triangular" cut to remove the low-energy/low- $\theta$ noise, applied to select photons.

$e p \pi^{0}$ final state:

- $M M^{2}(e p)$, the missing mass squared of the ep system

- $\theta_{\pi^{0} X}$, the angle between the measured and calculated $\pi^{0}$

- $M(\gamma \gamma)$, the two-photon invariant mass 
As in the DVCS selection, the carbon data, scaled by the ratio of $\mathrm{NH}_{3}$ to carbon Faraday-cup counts, was subtracted from the $\mathrm{NH}_{3}$ data to remove possible smearing effects stemming from the nuclear background on the peaks to be fitted. The data and $\pi^{0}$ electroproduction Monte-Carlo simulation were fit separately, at the preliminary cuts stage (i.e. "DIS" cuts: $Q^{2}>1 \mathrm{GeV}^{2}, W>2 \mathrm{GeV}^{2}, Q^{2}>-t$ and a cut on $M M^{2}(e p)$ ). The obtained widths were compared to the widths of the distributions after all cuts were applied to check for possible correlation effects. There are three $\pi^{0}$ decay photon-detection topologies possible:

- both $\pi^{0}$ decay photons detected in the Inner Calorimeter (IC), "IC-IC topology"

- both $\pi^{0}$ decay photons detected in CLAS's Electromagnetic Calorimeter (EC), "EC-EC topology"

- one of the $\pi^{0}$ decay photons detected in the EC, the other in the IC, "EC-IC topology"

Each topology was considered separately. The next two sections detail the cut selection for each topology.

\subsubsection{IC-IC topology}

Figures 3.43 - 3.45 show the simulation and $\mathrm{NH}_{3}, \mathrm{C}$ and $\mathrm{NH}_{3}-\mathrm{C}$ data distributions and fits to the $e p \pi^{0}$ exclusivity variables for the case in which both photons were detected in the Inner Calorimeter (IC) for Run Period B (plots for Run Period A are shown in Appendix B). After $M M^{2}(e p)$ cut and the subtraction of the carbon data, the exclusivity variables show very clear peaks. The widths of these peaks do not show sizable changes before and after application of all cuts, and a quite good agreement was present between data and $е p \pi^{0}$ Monte-Carlo simulation. 

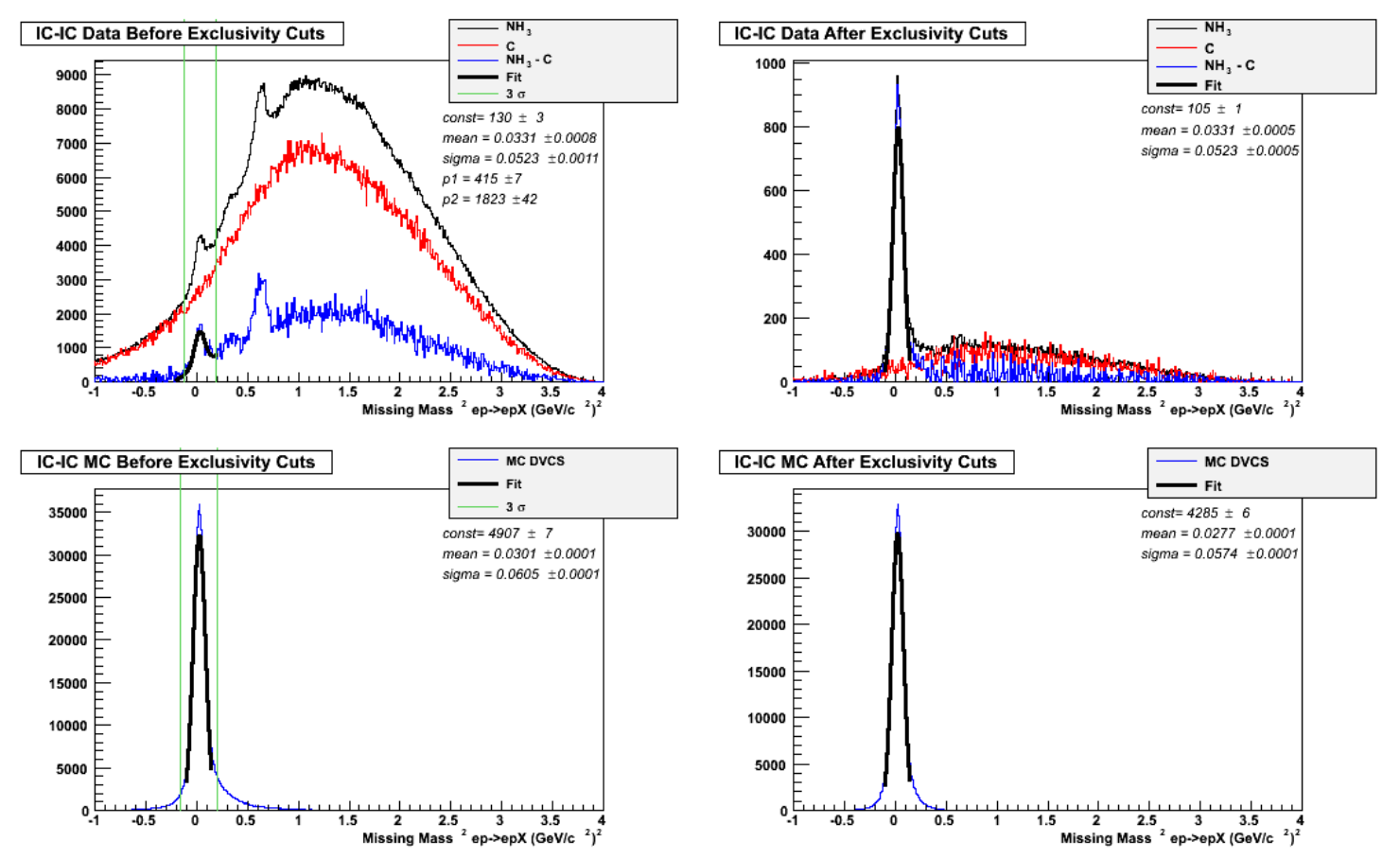

Fig. 3.43: Exclusive $\pi^{0}$ analysis Run Period B, IC-IC topology $M M^{2}(e p)$. Top plots: data (black: all $\mathrm{NH}_{3}$ data, red: charge scaled carbon data, blue: $\mathrm{NH}_{3}$ after carbon subtraction). Bottom plots: Monte-Carlo simulation for ep $\pi^{0}$. Left plots: without any but the DIS cuts; right plots: with all exclusivity cuts applied but the one on $M M^{2}(e p)$. 

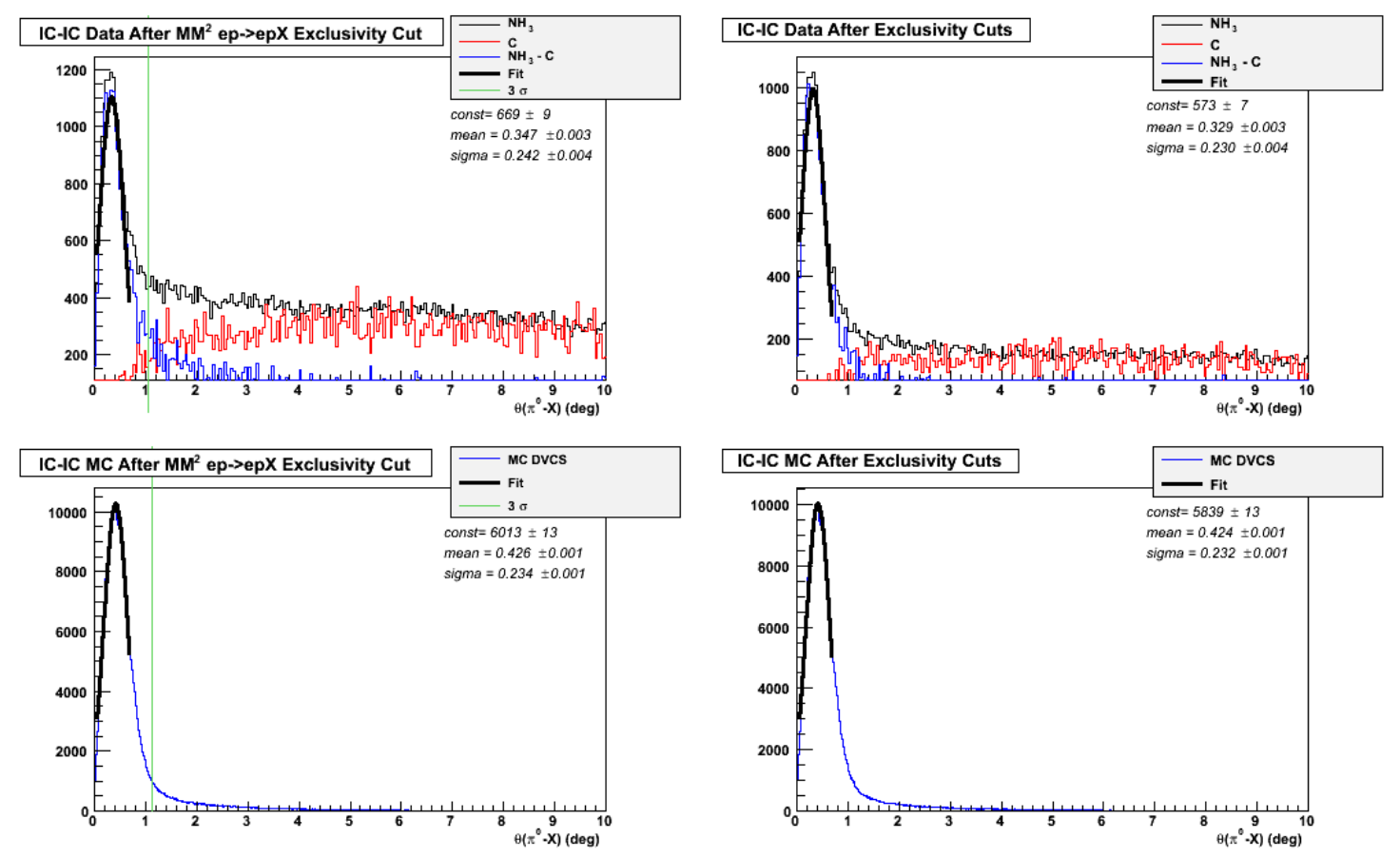

Fig. 3.44: Exclusive $\pi^{0}$ analysis Run Period B, IC-IC topology $\theta_{\pi^{0} X}$. Top plots: data (black: all $\mathrm{NH}_{3}$ data, red: charge scaled carbon data, blue: $\mathrm{NH}_{3}$ after carbon subtraction); bottom plots: Monte-Carlo simulation for ep $\pi^{0}$. Left plots: without any but the DIS and the $M M^{2}(e p)$ cuts; right plots: with all exclusivity cuts applied but the one on $\theta_{\pi^{0} X}$. 

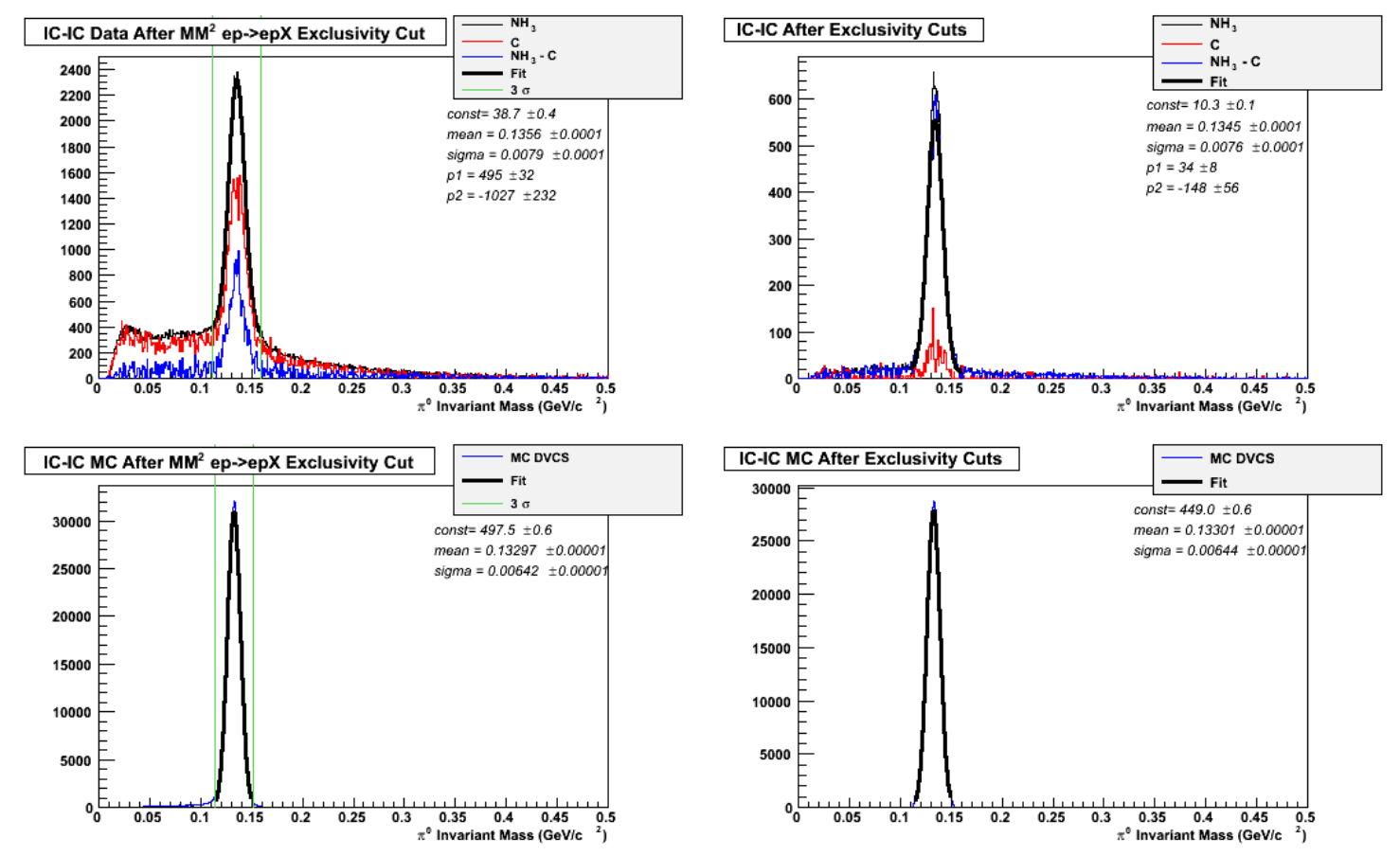

Fig. 3.45: Exclusive $\pi^{0}$ analysis Run Period B, IC-IC topology two-photons invariant mass $M(\gamma \gamma)$. Top plots: data (black: all $\mathrm{NH}_{3}$ data, red: charge scaled carbon data, blue: $\mathrm{NH}_{3}$ after carbon subtraction); bottom plots: Monte-Carlo simulation for $e p \pi^{0}$. Left plots: without any but the DIS and the $M M^{2}(e p)$ cuts; right plots: with all exclusivity cuts applied but the one on $M(\gamma \gamma)$. 


\subsubsection{EC-EC topology}

Figures 3.46 - 3.48 show the simulation and $\mathrm{NH}_{3}, \mathrm{C}$ and $\mathrm{NH}_{3}-\mathrm{C}$ data distributions and fits to the $e p \pi^{0}$ exclusivity variables for the case in which both photons were detected in CLAS's Electromagnetic Calorimeter (EC) for Run Period B (plots for Run Period A are shown in Appendix B). As with the IC-IC topology case, after the cut on $M M^{2}(e p)$, clear peaks appear in the other exclusivity variables.
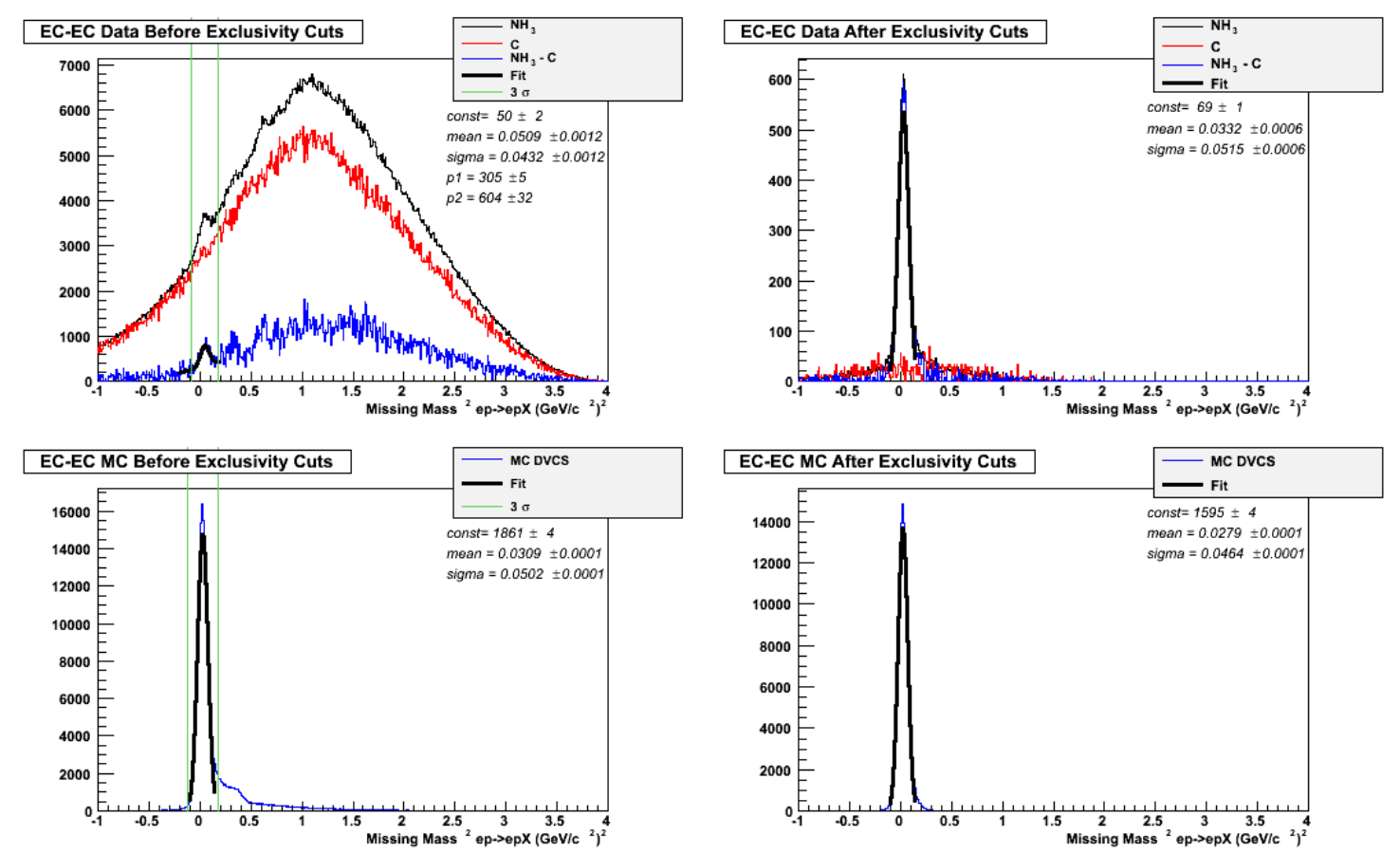

Fig. 3.46: Exclusive $\pi^{0}$ analysis Run Period B, EC-EC topology $M M^{2}(e p)$. Top plots: data (black: all $\mathrm{NH}_{3}$ data, red: charge scaled carbon data, blue: $\mathrm{NH}_{3}$ after carbon subtraction); bottom plots: Monte-Carlo simulation for $e p \pi^{0}$. Left plots: without any but the DIS cuts; right plots: with all exclusivity cuts applied but the one on $M M^{2}(e p)$. 

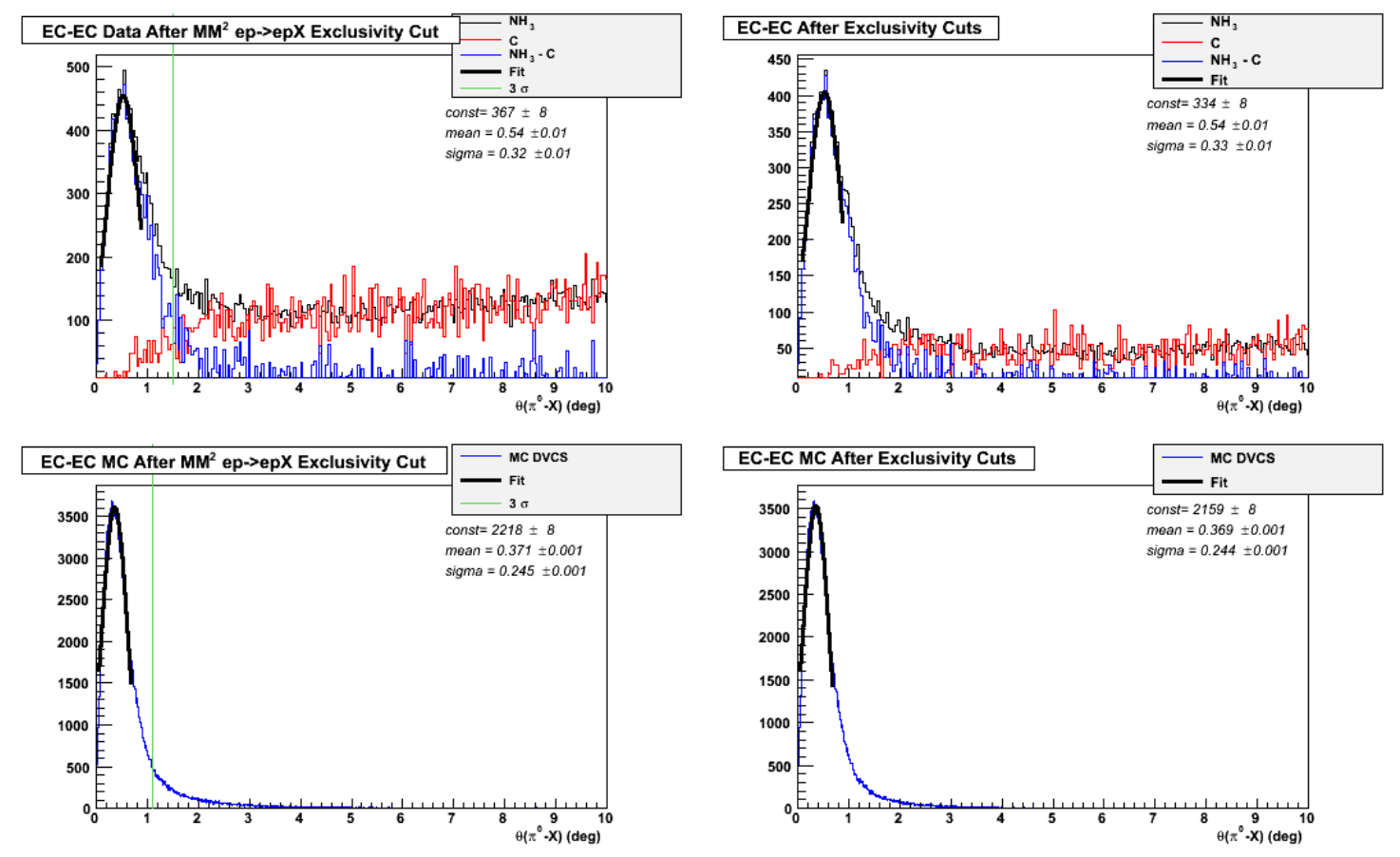

Fig. 3.47: Exclusive $\pi^{0}$ analysis Run Period B, EC-EC topology $\theta_{\gamma X}$. Top plots: data (black: all $\mathrm{NH}_{3}$ data, red: charge scaled carbon data, blue: $\mathrm{NH}_{3}$ after carbon subtraction); bottom plots: Monte-Carlo simulation for $e p \pi^{0}$. Left plots: without any but the DIS and the $M M^{2}(e p)$ cuts; right plots: with all exclusivity cuts applied but the one on $\theta_{\pi^{0} X}$. 

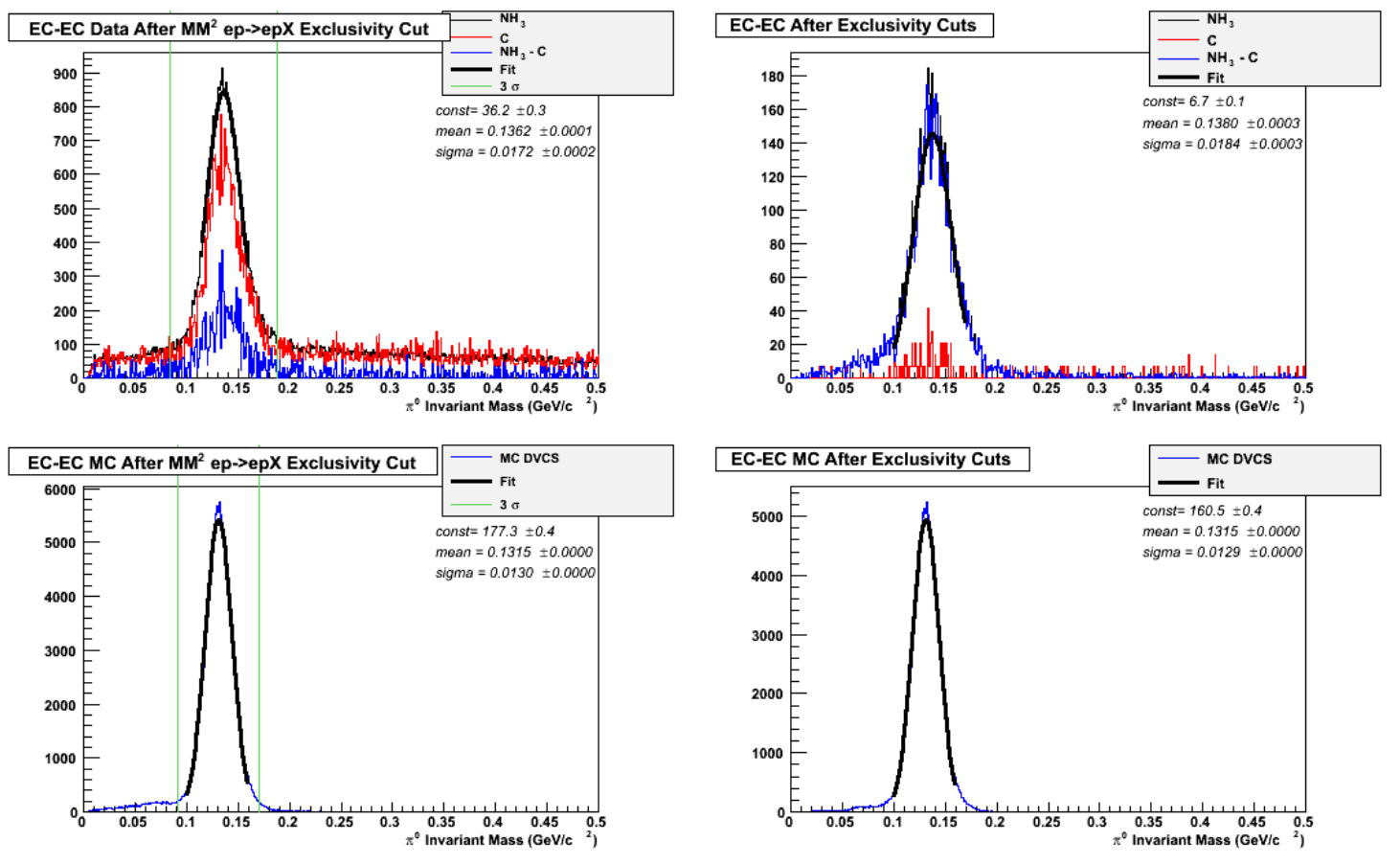

Fig. 3.48: Exclusive $\pi^{0}$ analysis Run Period B, EC-EC topology two-photons invariant mass $M(\gamma \gamma)$. Top plots: data (black: all $\mathrm{NH}_{3}$ data, red: charge scaled carbon data, blue: $\mathrm{NH}_{3}$ after carbon subtraction); bottom plots: Monte-Carlo simulation for $e p \pi^{0}$. Left plots: without any but the DIS and the $M M^{2}(e p)$ cuts; right plots: with all exclusivity cuts applied but the one on $M(\gamma \gamma)$. 


\subsubsection{EC-IC topology}

Figures 3.46 - 3.48 show the simulation and $\mathrm{NH}_{3}, \mathrm{C}$ and $\mathrm{NH}_{3}-\mathrm{C}$ data distributions and fits to the ep $\pi^{0}$ exclusivity variables for the case in which one decay photon was detected in the EC and one in the IC for Run Period B (plots for Run Period A are shown in Appendix B). In this case, the data distributions for $M M^{2}(e p)$ and $\theta_{\pi^{0} X}$ do not show any clear peak after carbon subtraction and DIS cuts. It was therefore decided, in this case, to adopt the cut widths obtained for the Monte-Carlo simulation. It must be noted, that this topology

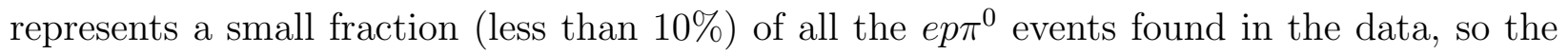
possible inaccuracies introduced by this method, which assumes a perfect match from data and simulation, do not impact strongly on the final results.
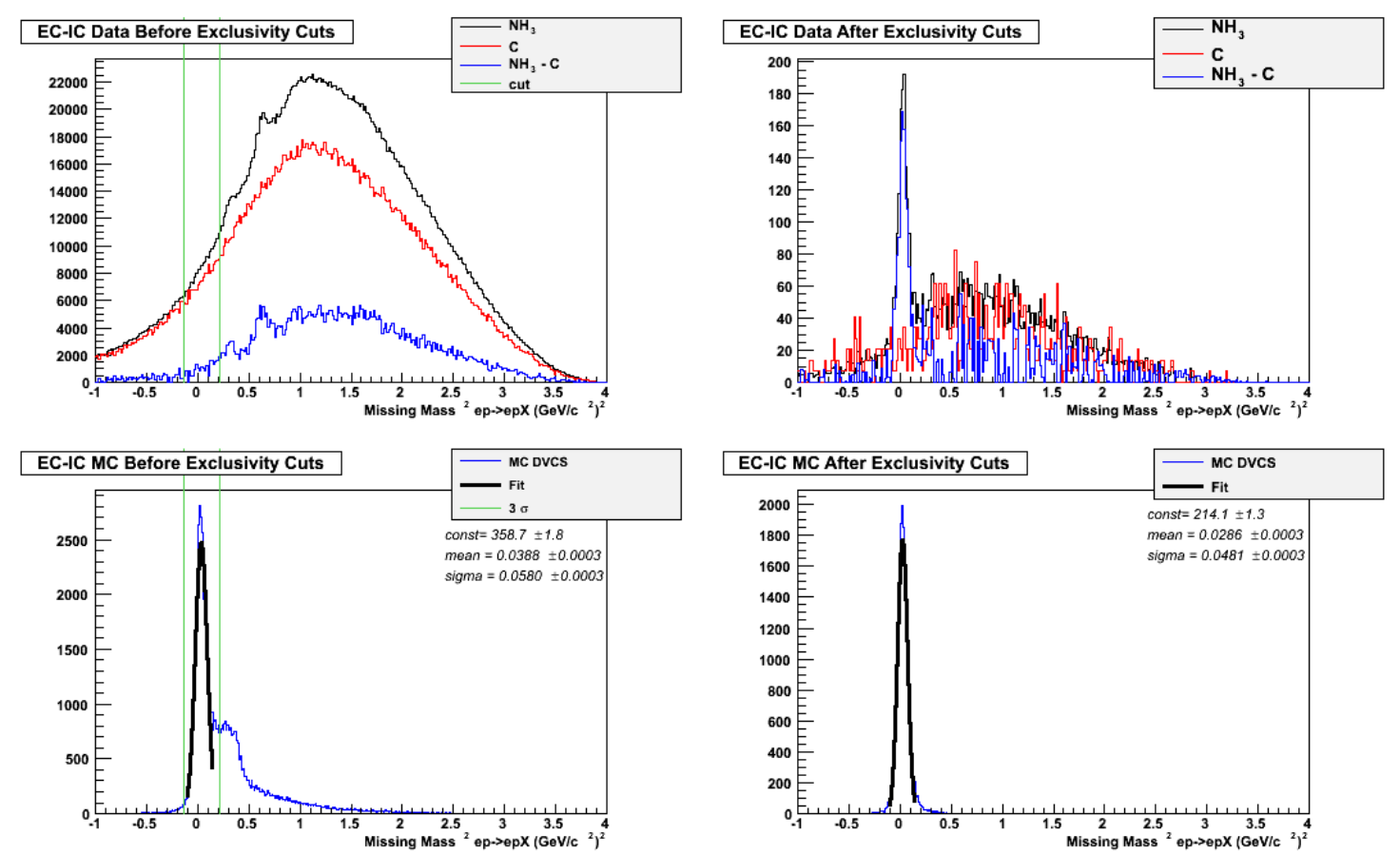

Fig. 3.49: Exclusive $\pi^{0}$ analysis Run Period B, EC-IC topology $M M^{2}(e p)$. Top plots: data (black: all $\mathrm{NH}_{3}$ data, red: charge scaled carbon data, blue: $\mathrm{NH}_{3}$ after carbon subtraction); bottom plots: Monte-Carlo simulation for ep $\pi^{0}$. Left plots: without any but the DIS cuts; right plots: with all exclusivity cuts applied but the one on $M M^{2}(e p)$. 

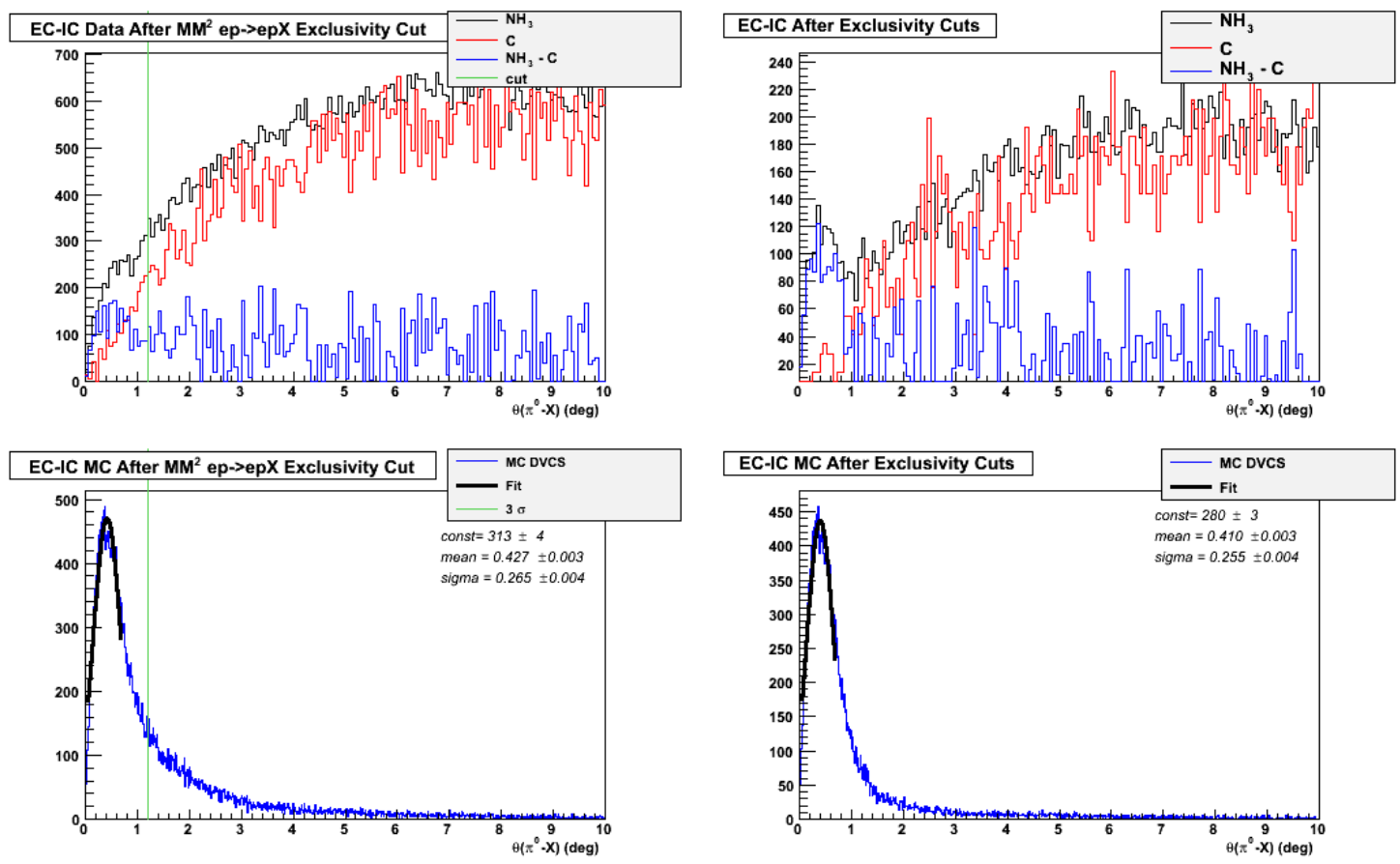

Fig. 3.50: Exclusive $\pi^{0}$ analysis Run Period B, EC-IC topology $\theta_{\gamma X}$. Top plots: data (black: all $\mathrm{NH}_{3}$ data, red: charge scaled carbon data, blue: $\mathrm{NH}_{3}$ after carbon subtraction); bottom plots: Monte-Carlo simulation for $e p \pi^{0}$. Left plots: without any but the DIS and the $M M^{2}(e p)$ cuts; right plots: with all exclusivity cuts applied but the one on $\theta_{\pi^{0} X}$. 

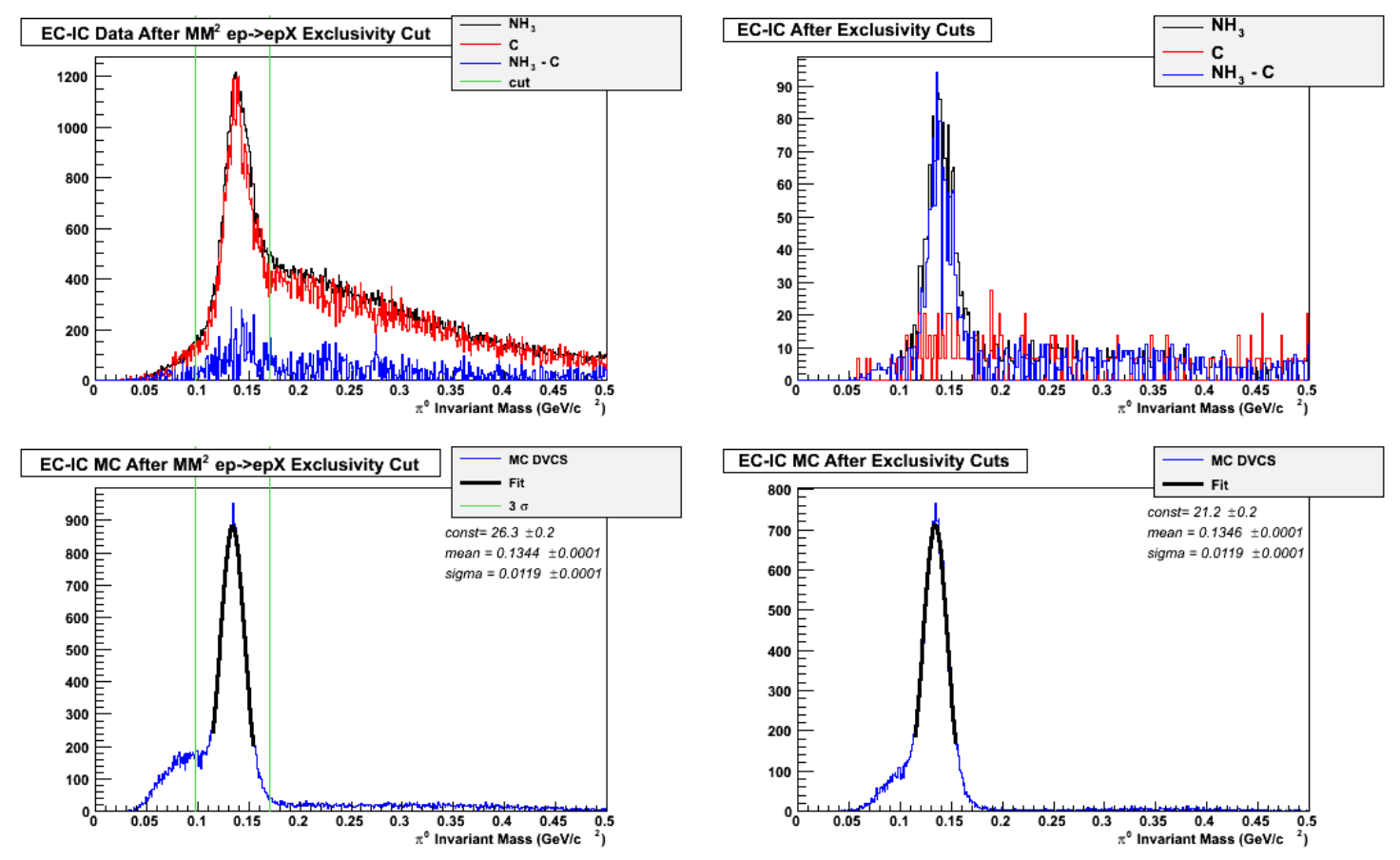

Fig. 3.51: Exclusive $\pi^{0}$ analysis Run Period B, EC-IC topology two-photons invariant mass $M(\gamma \gamma)$. Top plots: data (black: all $\mathrm{NH}_{3}$ data, red: charge scaled carbon data, blue: $\mathrm{NH}_{3}$ after carbon subtraction); bottom plots: Monte-Carlo simulation for $e p \pi^{0}$. Left plots: without any but the DIS and the $M M^{2}(e p)$ cuts; right plots: with all exclusivity cuts applied but the one on $M(\gamma \gamma)$. 


\subsection{Four-dimensional binning}

The DVCS reaction final state has three particles (9 unknowns), energy-momentum conservation provides 4 constraints, invariance for rotations around the azymuthal angle of the electron provides another constraint, so the reaction can be described by four independent kinematic variables $(9-5=4)$. The typical variables used to interpret the results in terms of Generalized Parton Distributions are:

- $x_{B}$, the standard Bjorken variable discussed in Section 1.3.

- $Q^{2}$, photon virtuality, $Q^{2}=-q^{2}$ (with $q=\vec{e}-\vec{e}^{\prime}$, the momentum of the virtual photon).

- $-t=-\left(\vec{p}^{\prime}-\vec{p}\right)^{2}$ (the squared 4-momentum transfer between the final and initial nucleon).

- $\phi$, the angle between the leptonic and the hadronic plane

In accordance to the choice made in previous DVCS analyses [15] [45], the binning of the data in the $Q^{2}-x_{B}$ plane was done making slices in $x_{B}$ and the $\theta$ angle of the electron (following the shape of the CLAS acceptance), shown in Figure 3.52. The limits and data-average centers of the slices are given in Table 3.9. The size of the bins was optimized to have comparable statistics in each bin. For each bin in $Q^{2}-x_{B}, 10$ equally-spaced bins in $\phi$ and 4 bins in $-t$ (limits and data-average centers are summarized in Table 3.10) were used. Figure 3.53 shows the binning in the $t-x_{B}$ plane.

\begin{tabular}{|c|c|c|c|c|}
\hline Bin number & $x_{B}$ bin & $\theta_{e}$ bin & $<x_{B}>$ & $<Q^{2}>\left(\mathrm{GeV}^{2}\right)$ \\
\hline 1 & $0.1<x_{B}<0.2$ & $15^{\circ}<\theta_{e}<48^{\circ}$ & 0.179 & 1.521 \\
2 & $0.2<x_{B}<0.3$ & $15^{\circ}<\theta_{e}<34^{\circ}$ & 0.256 & 1.972 \\
3 & $0.2<x_{B}<0.3$ & $34^{o}<\theta_{e}<48^{\circ}$ & 0.256 & 2.412 \\
4 & $0.3<x_{B}<0.4$ & $15^{\circ}<\theta_{e}<45^{\circ}$ & 0.345 & 2.601 \\
5 & $0.4<x_{B}<0.5$ & $15^{\circ}<\theta_{e}<45^{\circ}$ & 0.453 & 3.313 \\
\hline
\end{tabular}

Table 3.9: Definition of the bins in $x_{B}$ and $\theta_{e}\left(Q^{2}\right)$. 


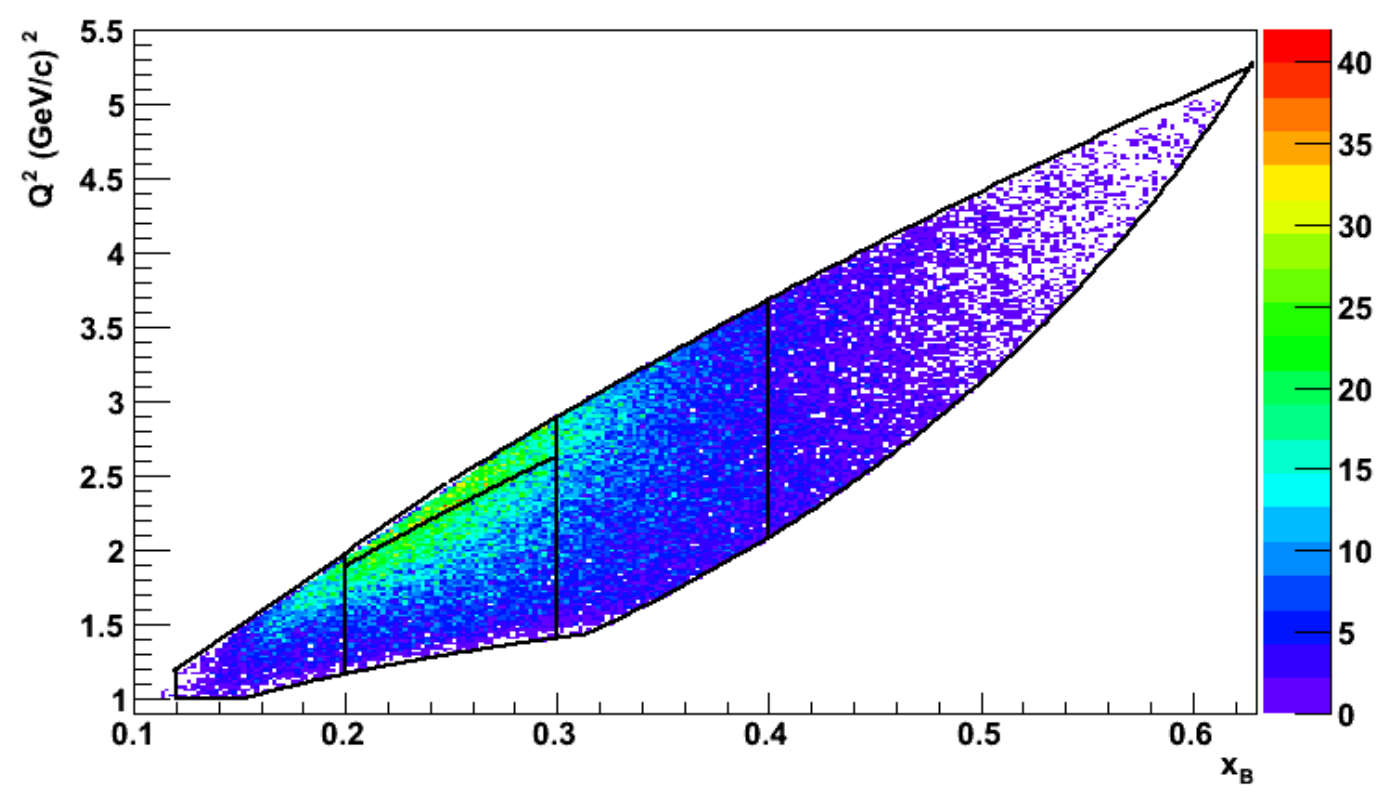

Fig. 3.52: Grid showing the $Q^{2}-x_{B}$ binning.

\begin{tabular}{|c|c|c|}
\hline Bin number & $-t$ bin & $<-t>\left(\mathrm{GeV}^{2}\right)$ \\
\hline 1 & $0.08<-t<0.18 \mathrm{GeV}^{2}$ & 0.137 \\
2 & $0.18<-t<0.3 \mathrm{GeV}^{2}$ & 0.234 \\
3 & $0.3<-t<0.7 \mathrm{GeV}^{2}$ & 0.467 \\
4 & $0.7<-t<2.0 \mathrm{GeV}^{2}$ & 1.175 \\
\hline
\end{tabular}

Table 3.10: Definition of the bins in $-t$. 


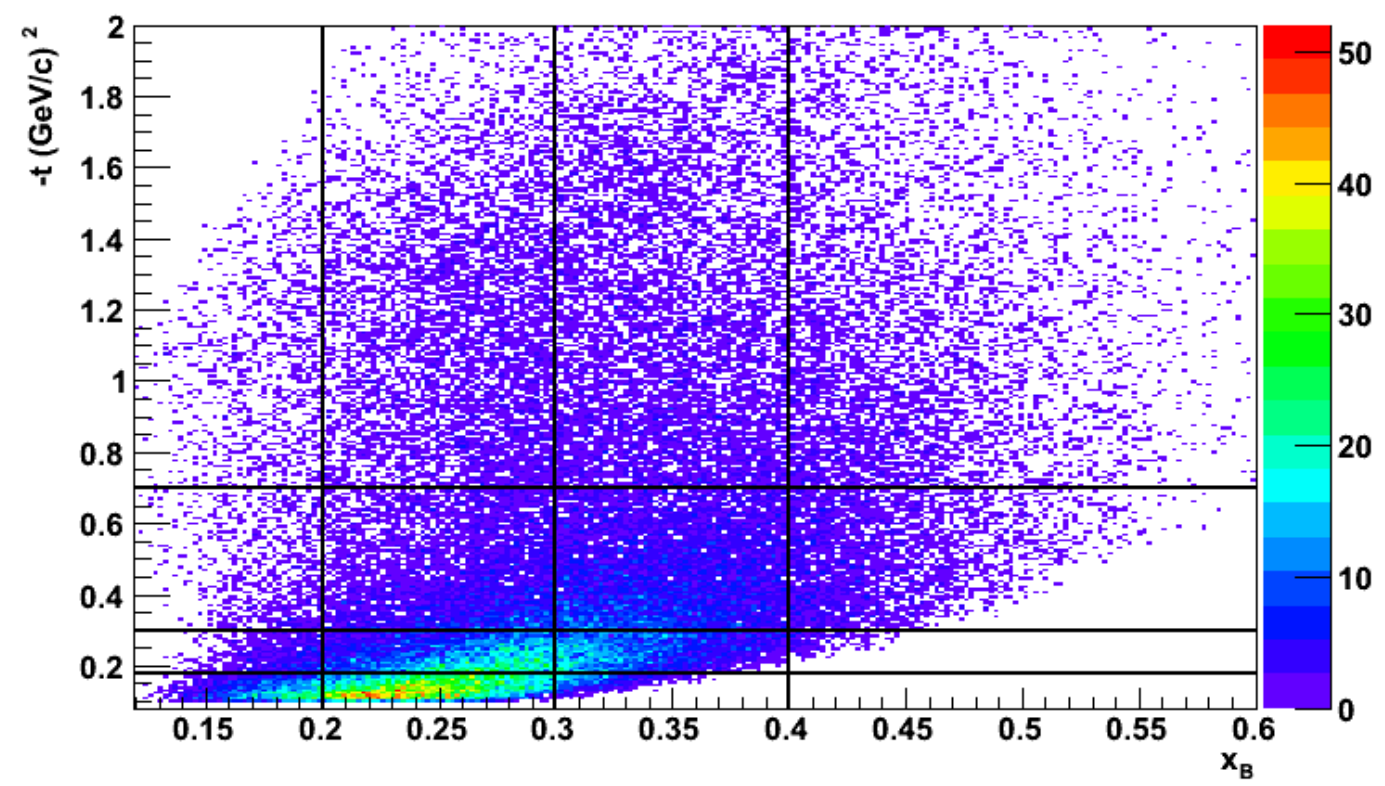

Fig. 3.53: Grid showing the $-t-x_{B}$ binning. 


\section{Chapter 4}

\section{Data Analysis}

\subsection{Electron Beam Energy}

Accurate beam energy, $E_{0}$, is needed to construct the incoming electron's 4-vector for use in exclusive event selection. To determine $E_{0}$ for each run period of the EG1-DVCS experiment, ep elastic events were selected and the beam energy determined by 2-body kinematics using the scattered electron and proton azimuthal angles with respect to the beam line, $\theta_{e}$ and $\theta_{p}$, as in Equation 4.1 (where $M_{p}$ is the proton mass).

$$
E_{0}=M_{p}\left\{\left[\tan \left(\theta_{e} / 2\right) \tan \left(\theta_{p}\right)\right]^{-1}-1\right\}
$$

A study done by A. Kim [46] was performed without raster or energy corrections (Sections 4.3 and 4.5) using the beam energy estimation from the Machine Control Center (MCC) for elastic selection cuts. An additional study was preformed using raster and energy corrections, detailed particle identification (Section 3.2) and elastic event selection (Section 3.3). Because of the low statistics for elastic events, groups of 10 "good" runs (see Section 3.1) were combined to make 19 groups of runs for Run Period A and 42 groups of runs for Run Period B. The beam energy was determined separately for each group by fitting the $E_{0}$ distribution with a Gaussian. Then, an average was determined from the means of the Gaussian fits. The $E_{0}$ mean and associated fit error for each group of runs during Run Periods A and B are shown in Figures 4.1 and 4.2. The average $\sigma$ for the separate $E_{0}$ distributions was 0.03 $G e V$; the mean values for all the separate groups fell well within $1 \sigma$. The resulting average beam energy is in agreement with the previous results from A. Kim within $2 \mathrm{MeV}$ as shown

in Table 4.1. Also shown in that table are the beam energy estimates given by Hall A arc measurements. The ep elastic measurement results are systematically lower than the Hall A 
measurements by amounts ranging from 6 to $15 \mathrm{MeV}$. Since the $e p$ elastic measurements correspond, on average, to vertex positions in the center of the target, part of this difference is due to the $3 \mathrm{MeV}$ ionization energy loss going through half of the target material. The remaining 3 to $12 \mathrm{MeV}$ can be partly attributed to the average amount of energy loss due to Bremsstrahlung of soft photons [46].

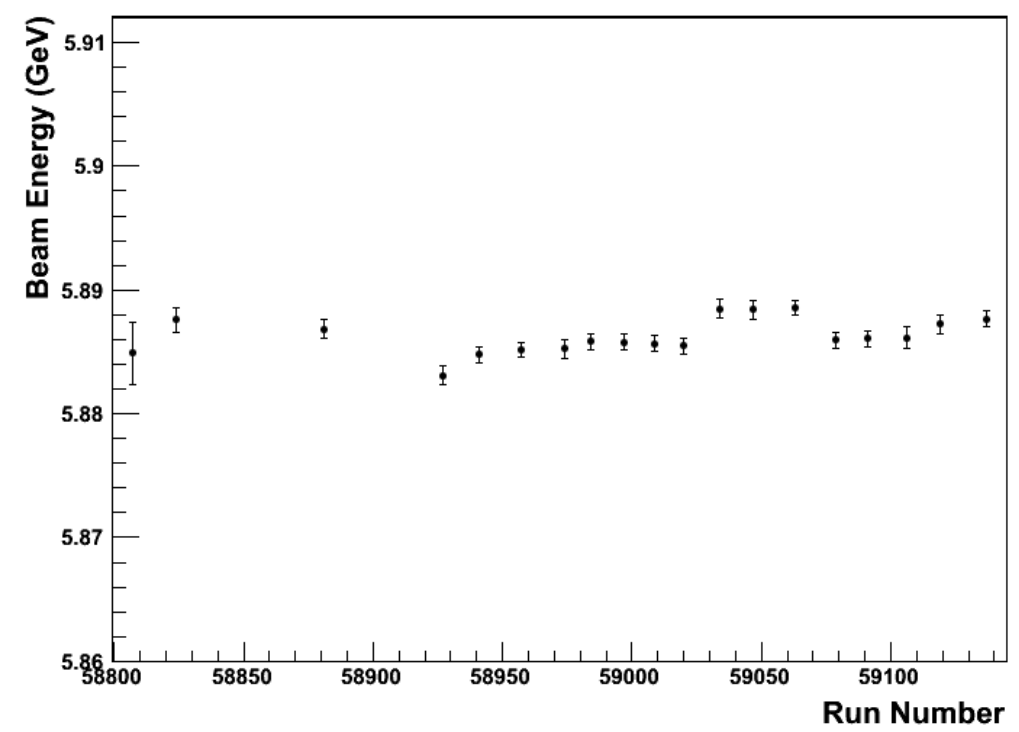

Fig. 4.1: Beam energy, $E_{0}$, from ep elastic analysis during Run Period A of the EG1-DVCS experiment.

\begin{tabular}{|c|c|c|c|}
\hline Run Period & $\begin{array}{c}\text { Beam Energy }(G e V) \\
\text { from Hall A }\end{array}$ & $\begin{array}{c}\text { Beam Energy }(\mathrm{GeV}) \\
\text { ep elastic analysis }\end{array}$ & $\begin{array}{l}\text { Beam Energy }(G e V) \\
\text { final ep elastic analysis }\end{array}$ \\
\hline $\mathrm{A}$ & $5.892(0.005)$ & $5.887(0.004)$ & $5.886(0.004)$ \\
\hline B & $5.967(0.005)$ & $5.954(0.004)$ & $5.952(0.004)$ \\
\hline
\end{tabular}

Table 4.1: Summary of the Hall B average electron beam energy during Eg1-DVCS Run Periods A and B through Hall A arc measurements, ep elastic analysis done previously by Andrey Kim [46], and ep elastic analysis done as part of this analysis (bold). Systematic errors are given in parentheses. 


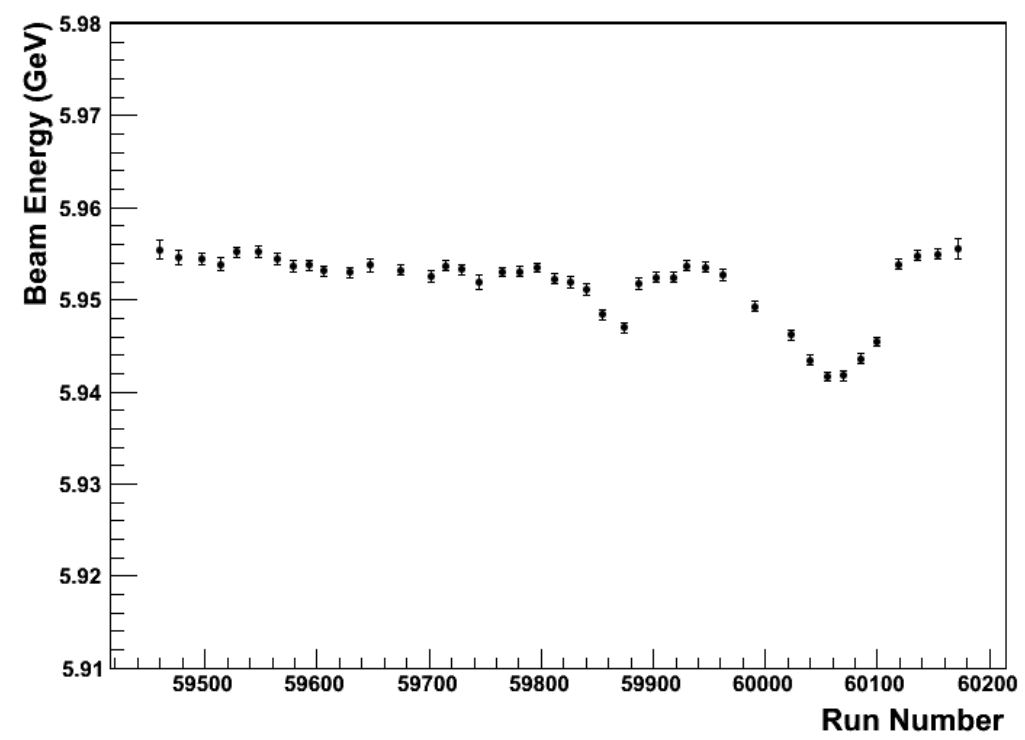

Fig. 4.2: Beam energy, $E_{0}$, from ep elastic analysis during Run Period B of the EG1-DVCS experiment. 


\subsection{Proton Polarization Using Elastic Asymmetry}

The procedure for extracting the proton polarization from the data starts with the determination of the product of the electron beam and proton target polarizations, $\mathrm{P}_{b} \mathrm{P}_{t}$. This product is found by comparing the measured ep elastic electron-spin asymmetry for a given proton polarization, $\mathrm{A}_{\text {measured }}$, with the known theoretical value, $\mathrm{A}_{\text {theory }}$ as in Equation 4.2. The expressions for the measured and theoretical asymmetries are given in Equation 4.3 and Equation 4.4 respectively.

$$
\begin{aligned}
& P_{b} P_{t}=\frac{A_{\text {measured }}}{A_{\text {theory }}} \\
& A_{\text {measured }}=\frac{N^{\uparrow \Uparrow}-N^{\downarrow \Uparrow}}{D_{f}\left(N^{\uparrow \Uparrow}+N^{\downarrow \Uparrow}\right)}
\end{aligned}
$$

For Equation 4.3, $\mathrm{N}$ is the normalized number of elastic events with beam polarization $(\uparrow / \downarrow)$ target polarization $\left(\Uparrow / \Downarrow\right.$ ), and dilution factor $D_{f}$ (discussed in Section 4.2.2). To normalize the data, the target polarization event counts are divided by the respective total accumulated charge. The theoretical asymmetry is given as [47]:

$$
A_{\text {theory }}=\frac{\cos \theta_{\gamma} \sqrt{1-\epsilon^{2}} A_{1}+\sqrt{2 \epsilon(1-\epsilon)} \sin \theta_{\gamma} A_{2}}{1+\epsilon\left(\frac{Q^{2}}{4 M^{2}}\right)^{-1}\left(\frac{G_{E}^{p}}{G_{M}^{P}}\right)^{2}}
$$

with $\theta_{\gamma}$ as the angle of the virtual photon with respect to the beam line, and

$$
\begin{aligned}
\epsilon & =\frac{1}{1+2\left(1+\frac{Q^{2}}{4 M^{2}}\right) \tan ^{2} \frac{\theta_{e}}{2}} \\
A_{1} & =1 \\
A_{2} & =\left(\sqrt{\frac{Q^{2}}{4 M^{2}}}\right)^{2} \frac{G_{E}^{p}}{G_{M}^{p}}
\end{aligned}
$$

where $\theta_{e}$ is the electron scattering angle, and the ratio of form factors [48]:

$$
\frac{G_{E}^{p}}{G_{M}^{p}}=\frac{1}{\mu_{p}}\left(1-\frac{Q^{2}}{9}\right)
$$

where $\mu_{p}$ is the proton magnetic moment.

\subsubsection{Theoretical Asymmetry}

The Asymmetry given in Equation 4.4. has a strong dependence on $\mathrm{Q}^{2}$, shown in Figure 4.3 . 


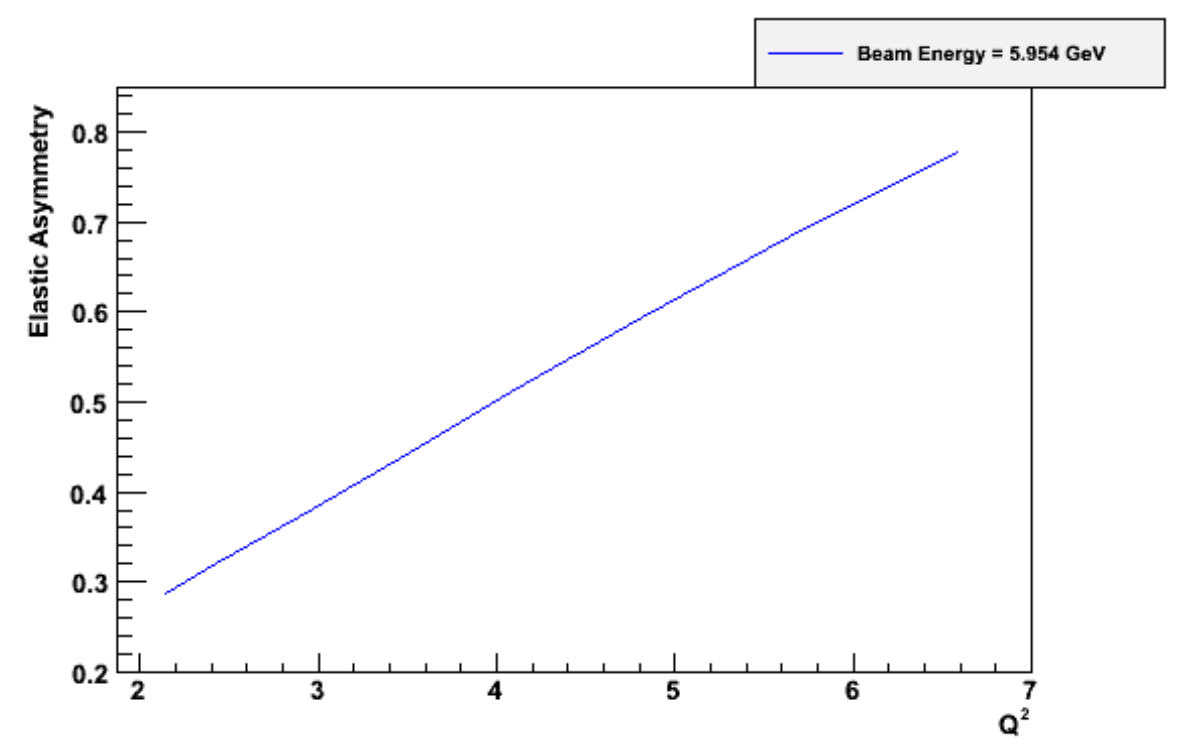

Fig. 4.3: $\mathrm{A}_{\text {theory }}$ plotted as a function of $\mathrm{Q}^{2}$ for Run Period B's beam energy.

The two Run Periods (A and B), which had different average beam polarizations $\left(P_{b}\right)$, were analyzed separately, in 8 bins of $\mathrm{Q}^{2}$. The theoretical asymmetry was calculated for each bin's average value of $Q^{2}$ as listed in Tables 4.2, 4.3.

\begin{tabular}{|c|c|c|}
\hline$Q^{2}$ range & $<Q^{2}>$ & $A_{\text {theo }}$ \\
\hline \hline $1.87-2.23$ & $\mathrm{~N} / \mathrm{A}$ & $\mathrm{N} / \mathrm{A}$ \\
$2.23-2.66$ & 2.56 & 0.338 \\
$2.66-3.17$ & 2.94 & 0.383 \\
$3.17-3.79$ & 3.44 & 0.442 \\
$3.79-4.52$ & 4.09 & 0.518 \\
$4.52-5.40$ & 4.89 & 0.609 \\
$5.40-6.45$ & 5.79 & 0.707 \\
$6.45-7.70$ & 6.62 & 0.789 \\
\hline
\end{tabular}

Table 4.2: List of the $Q^{2}$ bins, the average value of $Q^{2}$ for data in each bin, and the theoretical values of the asymmetry for Run Period A, $E_{\text {beam }}=5.887 \mathrm{GeV}$. 


\begin{tabular}{|c|c|c|}
\hline$Q^{2}$ range & $<Q^{2}>$ & $A_{\text {theo }}$ \\
\hline \hline $1.87-2.23$ & 2.14 & 0.286 \\
$2.23-2.66$ & 2.43 & 0.320 \\
$2.66-3.17$ & 2.89 & 0.372 \\
$3.17-3.79$ & 3.46 & 0.439 \\
$3.79-4.52$ & 4.09 & 0.512 \\
$4.52-5.40$ & 4.87 & 0.600 \\
$5.40-6.45$ & 5.83 & 0.703 \\
$6.45-7.70$ & 6.65 & 0.784 \\
\hline
\end{tabular}

Table 4.3: List of the $Q^{2}$ bins, the average value of $Q^{2}$ for data in each bin, and the theoretical values of the asymmetry for Run Period B, $E_{\text {beam }}=5.954 \mathrm{GeV}$. 


\subsubsection{Elastic Dilution Factor}

To account for the molecular background associated with the ${ }^{14} \mathrm{NH}_{3}$ target material, runs on a ${ }^{12} \mathrm{C}$ target where taken periodically throughout the experiment. To study the dilution factor, the elastic cuts were applied to both ${ }^{12} \mathrm{C}$ and $\mathrm{NH}_{3}$ data. The ${ }^{12} \mathrm{C}$ and $\mathrm{NH}_{3}$ data were normalized using their associated accumulated charge. In addition, the carbon data was corrected by the factor $c$, defined in Equation 4.7, to take into account the different target lengths and materials for $\mathrm{NH}_{3}$ and ${ }^{12} \mathrm{C}$ runs [49],

$$
c=\frac{\left(\left(L-l_{N H_{3}}\right) * \rho_{H e}\right)+\left(l_{N H_{3}} * \rho_{N H_{3}} * 14 / 17\right)+\left(l_{K} * \rho_{K}\right)+\left(l_{A l} * \rho_{A l}\right)}{\left(\left(L-l_{C}\right) * \rho_{H e}\right)+\left(l_{C} * \rho_{C}\right)+\left(l_{K} * \rho_{K}\right)+\left(l_{A l} * \rho_{A l}\right)} \simeq 0.7
$$

with:

- $L$, the total length between the entrance and exit windows in the target chamber

- $l_{N H_{3}}, l_{C}, l_{K}, l_{A l}$, the packing fraction, or effective length of the ammonia target, carbon target, Kapton target cell covers, and aluminum target chamber windows respectively

- $\rho_{H e}, \rho_{N_{3}}, \rho_{C}, \rho_{K}, \rho_{A l}$, the volume densities of the liquid helium in the target chamber, ammonia target, carbon target, Kapton target cell covers, and aluminum target chamber windows respectively

The elastic dilution factor, $D_{f}$, that is defined in the same way as for the DVCS and $e p \pi^{0}$ channels, was computed for the two run periods of the experiment used in this analysis by using Equation 4.8 .

$$
D_{f}=\frac{N_{N H_{3}}-c * N_{C}}{N_{N H_{3}}}
$$

Where $\mathrm{N}_{\mathrm{NH}_{3}}$, and $\mathrm{N}_{\mathrm{C}}$ are the number of events normalized by their respective charge accumulation. The resulting dilution factors and associated statistical errors are listed in Table 4.4.

\subsubsection{PbPt Results}

The resulting $P_{b} P_{t}$ Values for the two different run periods can be seen in Figures 4.4 and 4.5, and summarized in Table 4.5. 


\begin{tabular}{|c|c|}
\hline Run Period & $D_{f}^{\text {elastic }}$ \\
\hline \hline $\mathrm{A}$ & $0.980 \pm 0.020$ \\
$\mathrm{~B}$ & $0.982 \pm 0.017$ \\
\hline
\end{tabular}

Table 4.4: ep elastic dilution results with statistical error for EG1-DVCS Run Periods A and B.

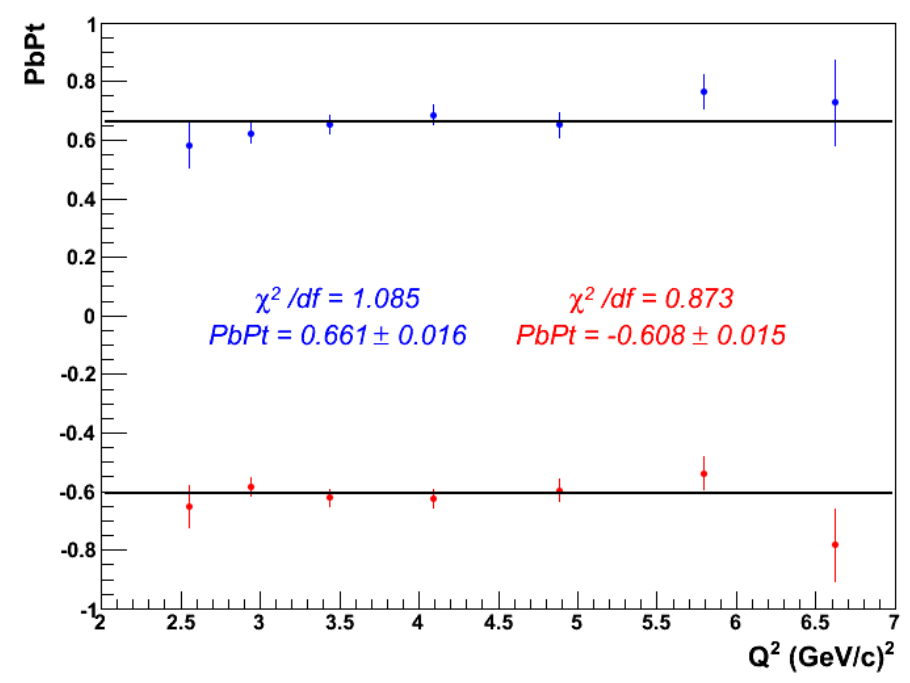

Fig. 4.4: $P_{b} P_{t}$ for 7 bins in $\mathrm{Q}^{2}$ for Run Period A data with beam energy $=5.887 \mathrm{GeV}$ for positive target polarization runs (blue) and negative target polarization runs (red).

\begin{tabular}{|l|c|c|}
\hline Run Period & $P_{b} P_{t}\left(+\mathrm{P}_{t}\right)$ & $P_{b} P_{t}\left(-\mathrm{P}_{t}\right)$ \\
\hline \hline $\mathrm{A}$ & $0.661 \pm 0.016$ & $-0.608 \pm 0.015$ \\
$\mathrm{~B}$ & $0.668 \pm 0.011$ & $-0.622 \pm 0.010$ \\
\hline
\end{tabular}

Table 4.5: $P_{b} P_{t}$ results with statistical error. 


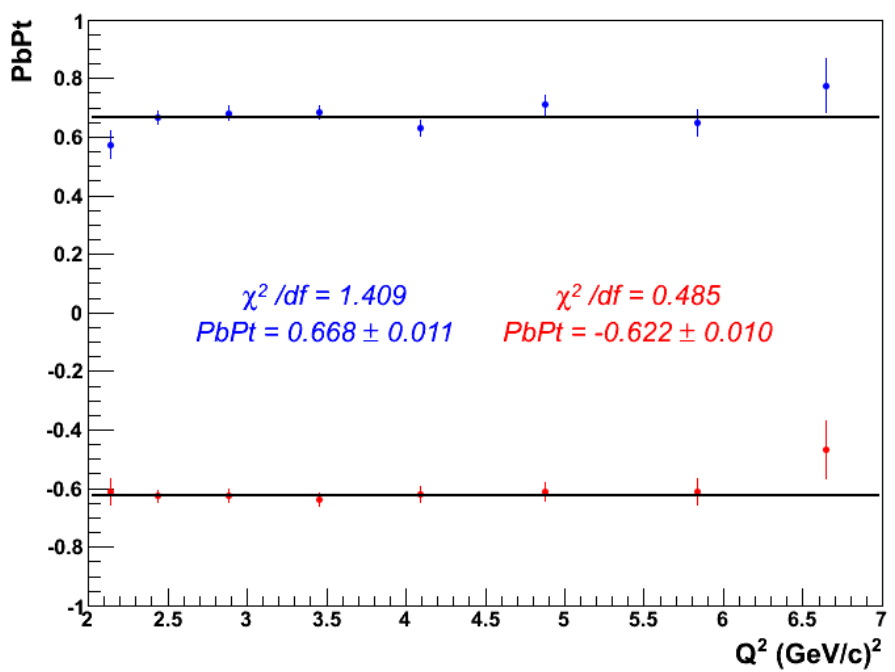

Fig. 4.5: $P_{b} P_{t}$ for 8 bins in $\mathrm{Q}^{2}$ for Run Period $\mathrm{B}$ data with beam energy $=5.954 \mathrm{GeV}$ for positive target polarization runs (blue) and negative target polarization runs (red). 


\subsubsection{Electron Polarization from Møller Measurement Results}

To determine the average electron beam polarization for each of the two EG1-DVCS run periods an average was taken of elastic events weighted with their respective Møller polarization value (Tables 2.2 and 2.3). The average for the electron beam polarization obtained with this method are, for Run Period A, 0.865 with a standard deviation of 0.035, and for Run Period B, 0.835 with a standard deviation of 0.017 . Two systematic studies were preformed on the Møller polarimeter during the EG1-DVCS experiment. These were to estimate the error associated with the certainty of the polarization of the Møller target in the two polarization configurations. This was done by taking Møller measurements with the half-wave plate in and out of the electron beam, thereby inverting the helicity state of the beam. The resulting systematic error was determined to be 0.02 .

\subsubsection{Target Polarization Results}

Table 4.6. shows the final average values of target polarization, $P_{t}$, obtained using elastic electron beam helicity asymmetry measurements (Section 4.2.3), along with the average of the Møller measurements of the electron beam polarization taken periodically throughout the experiment (Section 4.2.4). The uncertainty associated with the values of $P_{t}$ include the relative statistical error from the $P_{b} P_{t}$ measurement and the relative systematic error and standard deviation associated with the Møller measurements, and will be discussed further in Sections 5.6.1 and 5.6.2.

\begin{tabular}{|l|c|c|}
\hline Run Period & $+\mathrm{P}_{t}(\%)$ & $-\mathrm{P}_{t}(\%)$ \\
\hline \hline $\mathrm{A}$ & $76.41(74.74)$ & $-70.29(-68.77)$ \\
$\mathrm{B}$ & $80.00(77.76)$ & $-74.49(-72.41)$ \\
\hline
\end{tabular}

Table 4.6: Average proton target polarization, $P_{t}$, for Run Periods A and B. The values in parenthesis, which are used in the asymmetry calculation, are averages of the projection of target polarization on the virtual-photon direction. 


\subsection{Electron Beam Raster Correction}

Particle tracking in the CLAS reconstruction package reconstructs particles back to a nominal line through the center of CLAS. Because the EG1-DVCS electron beam was rastered around the center line, the average reconstructed vertex position, $v_{z}$, will be displaced. This displacement follows a sector dependent $\cos (\phi)$ distribution, where $\phi$ is the lab polar angle of the particle. To correct for this displacement in reconstructed vertex, a correspondence was determined by P. Bosted [50] between information from the raster magnets' ADC values, which are proportional to the current going through the raster magnets, and the actual beam position, found by making an average value of $v_{z}$ corrected for beam position. The correspondence was determined by minimizing $\chi^{2}$ as defined in Equation 4.9 using five parameters corresponding to the raster ADC offsets $x_{\text {off }}$ and $y_{o f f}$, the gain factors to convert ADC values $\left(R_{X}, R_{Y}\right)$ to $\mathrm{cm}, x_{\text {gain }}, y_{\text {gain }}$, and the average vertex position $z_{\text {nom }}$.

$$
\chi^{2}=\sum_{i=1}^{N}\left(z_{i}-z_{\text {nom }}\right)^{2}
$$

where the sum is over $N$ particles and

$$
z_{i}=z_{i}^{0}+\frac{x_{i}^{\prime}}{\tan \left(\theta_{i}\right)}
$$

where

$$
x_{i}^{\prime}=\frac{x_{i} \cos \left(\phi_{i}^{s}\right)+y_{i} \sin \left(\phi_{i}^{s}\right)}{\cos \left(\phi_{i}-\phi_{i}^{s}\right)}
$$

and where

$$
\begin{aligned}
\phi_{i}^{s} & =(\text { sector }-1)(\pi / 3) \\
x_{i} & =\left(R_{X}^{i}-x_{o f f}\right) x_{\text {gain }} \\
y_{i} & =\left(R_{y}^{i}-y_{o f f}\right) y_{\text {gain }}
\end{aligned}
$$

The five parameters were determined for each file of each run in the EG1-DVCS dataset, and average values were extracted for sets of runs that displayed similar results. From this correction, for each run, information on the raster $\mathrm{x}, \mathrm{y}$ position as well as the corrected nominal vertex was used for angular corrections discussed in Section 4.4. 


\subsection{Angular Correction}

Directional cosines (cy, cy, cz) are defined such that

$$
\begin{aligned}
p \cdot c x & =p_{x} \\
p \cdot c y & =p_{y} \\
p \cdot c z & =p_{z} \\
1 & =\sqrt{c x^{2}+c y^{2}+c z^{2}}
\end{aligned}
$$

The CLAS reconstruction package (Section 2.5) uses a field map of the target magnet along with the directional cosines from the Region 1 Drift Chambers to reconstruct a particle's directional cosines at the position of the target. An improvement was made to the determination of particle directional cosines by using the beam (x,y) position obtained with raster information (Section 4.3). The method, developed by P. Bosted [51], traces back from the Region 1 Drift Chambers using the above mentioned field map but forces the trajectory to stop exactly on the $(\mathrm{x}, \mathrm{y})$ coordinates of the beam, known from the raster information. In addition to improving, by a factor of two, the angular resolution, this process also results in a small improvement in the particle $z$ vertex position, $v_{z}$. See Reference [51] for a full description of the method.

In addition to making the angular corrections mentioned above, the angular deflections from the mis-aliment in angle of the target solenoid relative to the magnetic axis of the CLAS torus were determined and corrected for.

In practice, the target magnet axis can be slightly rotated by angles $c_{x}^{m}, c_{y}^{m}$ relative to the magnetic axis of the CLAS torus. This will result in transverse field components to the target magnetic field, $B_{x}=c_{x}^{m} B_{z}(0)$ and $B_{y}=c_{y}^{m} B_{z}(0)$, acting over an effective length $d=\int B_{z}(z) d z / B_{0}$. The effect of the transverse components can be written as Equation 4.14.

$$
\begin{aligned}
\delta c_{x} & =c_{z} A / p \\
\delta c_{y} & =c_{z} B / p
\end{aligned}
$$

where $p$ is the momentum of the particle, and $A$ and $B$ are unknowns. Determination of the $A$ and $B$ parameters was accomplished by minimizing the invariant mass of the products of photons that convert to electron-positron pairs. The invariant mass of the $e^{+} e^{-}$system, 
$M e^{+} e^{-}$, considering the two particles to be nearly massless (a valid approximation with the resolution of CLAS) is given by Equation 4.15.

$$
M e^{+} e^{-}=\sqrt{4 p_{e^{+}} p_{e}=\sin ^{2}\left(\frac{\theta_{e^{+} e^{-}}}{2}\right)}
$$

where $\theta_{e^{+} e^{-}}$is the opening angle between the positron and electron. The $A$ and $B$ parameters, as well as the resulting corrections $\delta c_{x}$ and $\delta c_{y}$ for the EG1-DVCS dataset Run Periods A and B were determined by P. Bosted [51] and are listed in Equation 4.16, with $q$ being the particle's charge.

$$
\begin{array}{r}
\delta c_{x}=0.0007 q c_{z} / p \\
\delta c_{y}=-0.0022 q c_{z} / p
\end{array}
$$

The effect of the angular correction is displayed in Figure 4.6 which shows the electron beam energy, $E_{0}$, from $e p$ elastic analysis using Equation 4.17, where $\theta_{e}$ and $\theta_{p}$ are the scattered electron and proton azimuthal angles with respect to the beam line, respectively, and $M_{p}$ is the proton mass. The black lines are $E_{0}$ determined using the CLAS reconstruction package directional cosines for the scattered electron and proton, and the red lines are $E_{0}$ determined using the above corrections to the directional cosines.

$$
E_{0}=M_{p}\left\{\left[\tan \left(\theta_{e} / 2\right) \tan \left(\theta_{p}\right)\right]^{-1}-1\right\}
$$



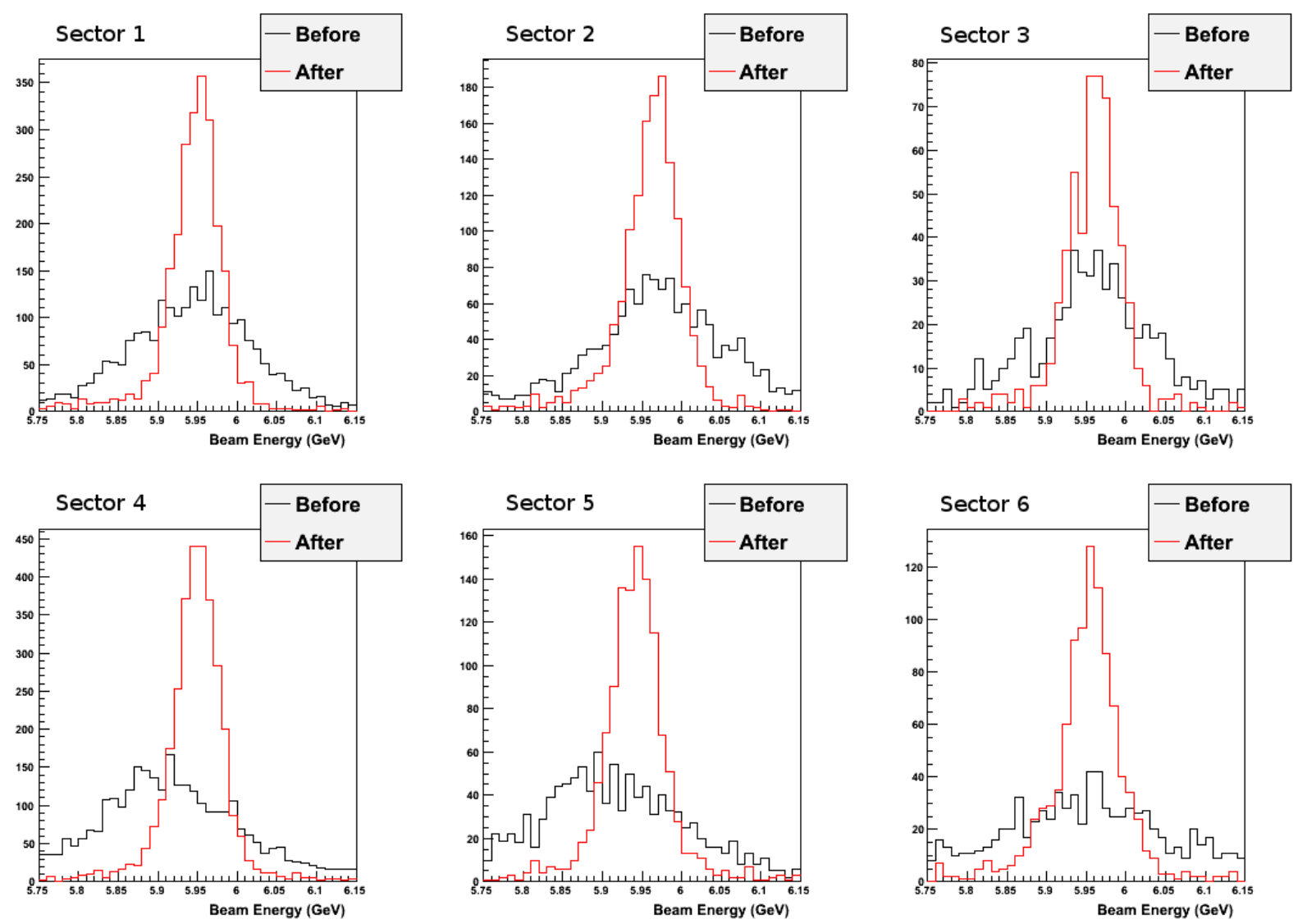

Fig. 4.6: Beam energy, $E_{0}$, for each sector in CLAS from ep elastic analysis for a group of typical runs in Run Period B of the EG1-DVCS experiment before (black) and after (red) angular corrections. 


\subsection{Momentum Correction}

As scattered particles traverse through the target and the target chamber, out to the first drift chamber, they experience energy loss through ionization that is not accounted for in the CLAS reconstruction package. Monte-Carlo simulation was used to compute "energy-loss" corrections to the momenta of electrons and protons. The corrections were calculated by fitting the ratio of generated and reconstructed momentum, as a function of momentum. Because the ratio of momenta is momentum dependent, the ratios were plotted for 15 slices in momentum, and then each distribution fitted with a Gaussian. The mean positions of the Gaussians were then fitted with Equation 4.18 for protons and Equation 4.19 for electrons. The resulting fit can be seen in the top left plots of Figure 4.7 for protons, and Figure 4.8 for electrons. The associated correction factors are listed in Tables 4.7 (Run Period A) and 4.8 (Run Period B).

$$
\begin{gathered}
p_{\text {corr }}=p \cdot\left(a+b / p-c / p^{2}+d / p^{3}\right), \\
p_{\text {corr }}=p \cdot\left(a+b / p-c / p^{2}\right) .
\end{gathered}
$$

\begin{tabular}{|l|c|c|}
\hline Parameter & Proton & Electron \\
\hline \hline a & 0.997204 & 0.999452 \\
b & 0.00562253 & 0.00615979 \\
c & 0.00348868 & 0.00304068 \\
d & 0.00229922 & \\
\hline
\end{tabular}

Table 4.7: Parameters for the momentum corrections for protons and electrons, EG1-DVCS Run Period A. 

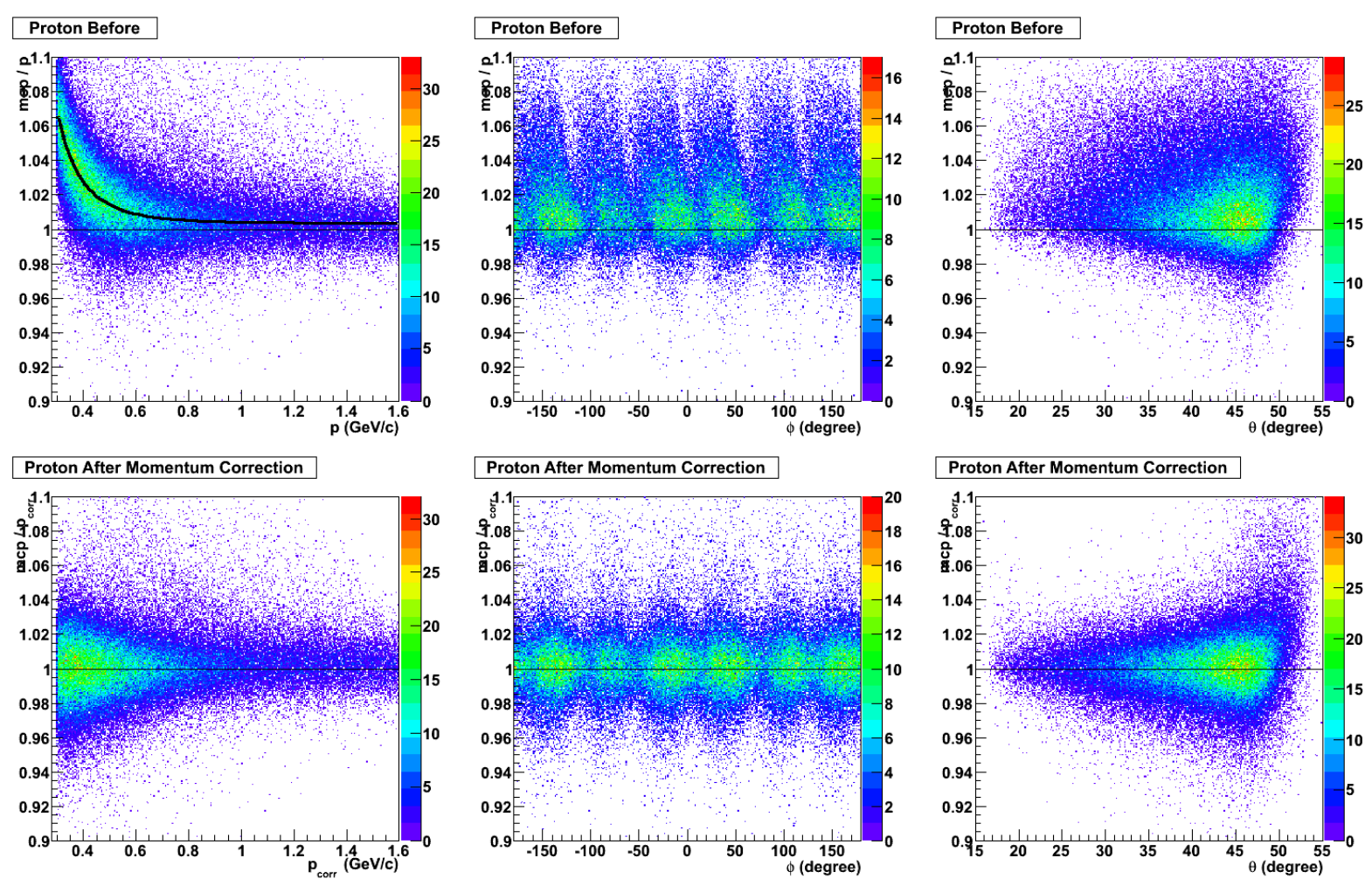

Fig. 4.7: Proton energy loss correction, Monte-Carlo DVCS simulation. Generated momentum divided by the reconstructed one, as a function of momentum (left), $\phi$ (middle), $\theta$ (right). Top: before corrections. Bottom: after corrections. The black curve in the upper left plot is the fit to the means of Gaussian fits in momentum slices.

\begin{tabular}{|l|l|l|}
\hline Parameter & Proton & Electron \\
\hline \hline a & 0.992767 & 0.9997360 \\
b & 0.0147232 & 0.0105136 \\
c & 0.0084665 & 0.00515416 \\
d & 0.0032769 & \\
\hline
\end{tabular}

Table 4.8: Parameters for the momentum corrections for protons and electrons, EG1-DVCS Run Period B. 

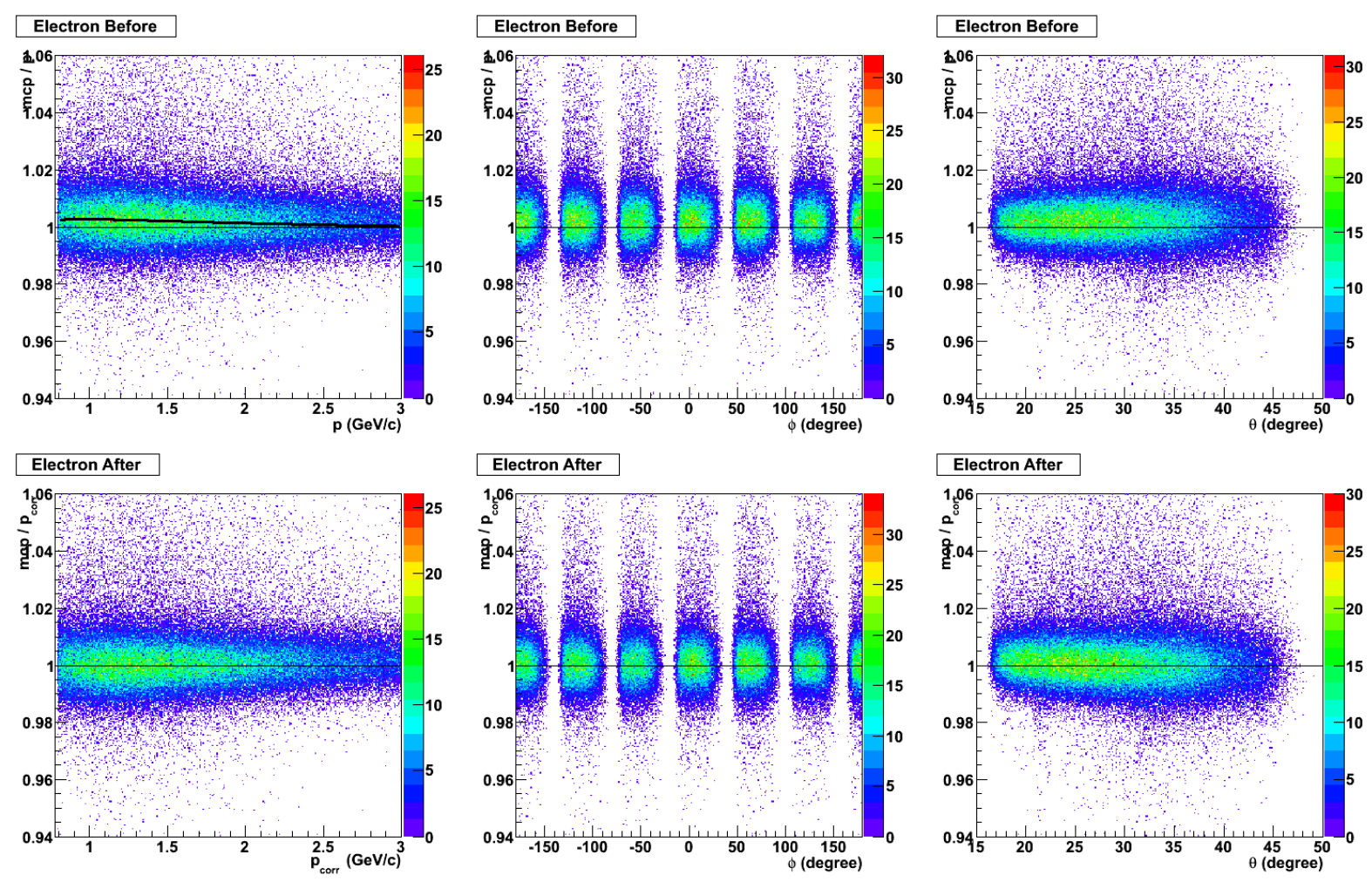

Fig. 4.8: Electron energy loss correction, Monte-Carlo DVCS simulation. Generated momentum divided by the reconstructed one, as a function of momentum (left), $\phi$ (middle), $\theta$ (right). Top: before corrections. Bottom: after corrections. The black curve in the upper left plot is the fit to the means of Gaussian fits in momentum slices. 
After the corrected momenta were computed, the angle corrections of Section 4.4 were re-applied. The effect of the energy loss corrections can be seen in Figures 4.9 and 4.10. The momentum and $t$ dependence of the DVCS electron-proton squared missing mass, $M M^{2}(e p)$, is strongly reduced after application of the corrections. Instead, the corrections do not appear to bring any sizable reduction to the width of the $M M^{2}(e p)$ peak, apart from the two lowest- $t$ bins. This is consistent with the fact that the correction is bigger at low proton momenta, corresponding to low $-t$ values.
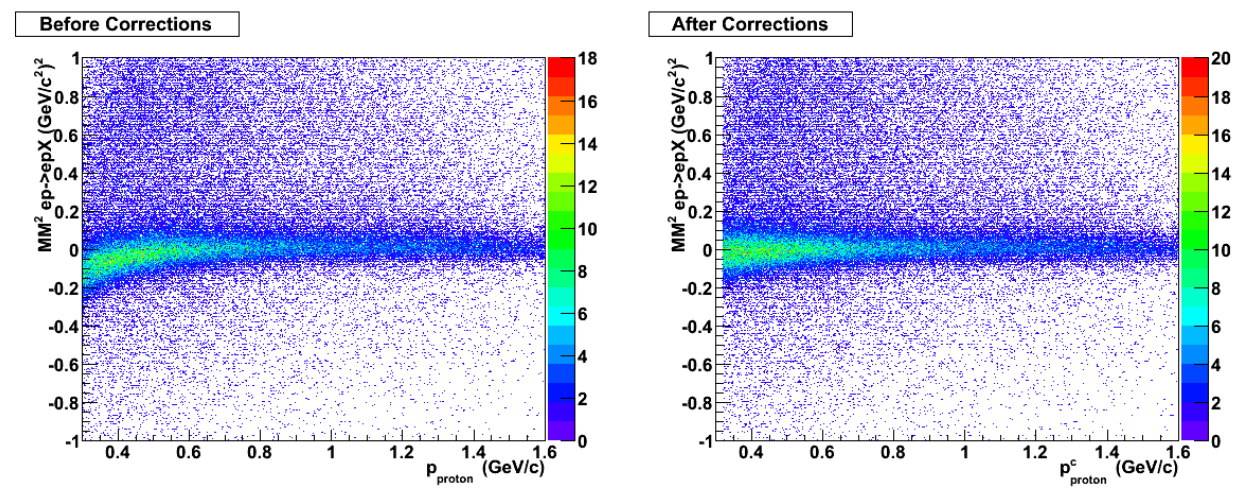

Fig. 4.9: Squared missing mass of the ep system in data, as a function of proton momentum, before (left) and after (right) energy-loss corrections. 


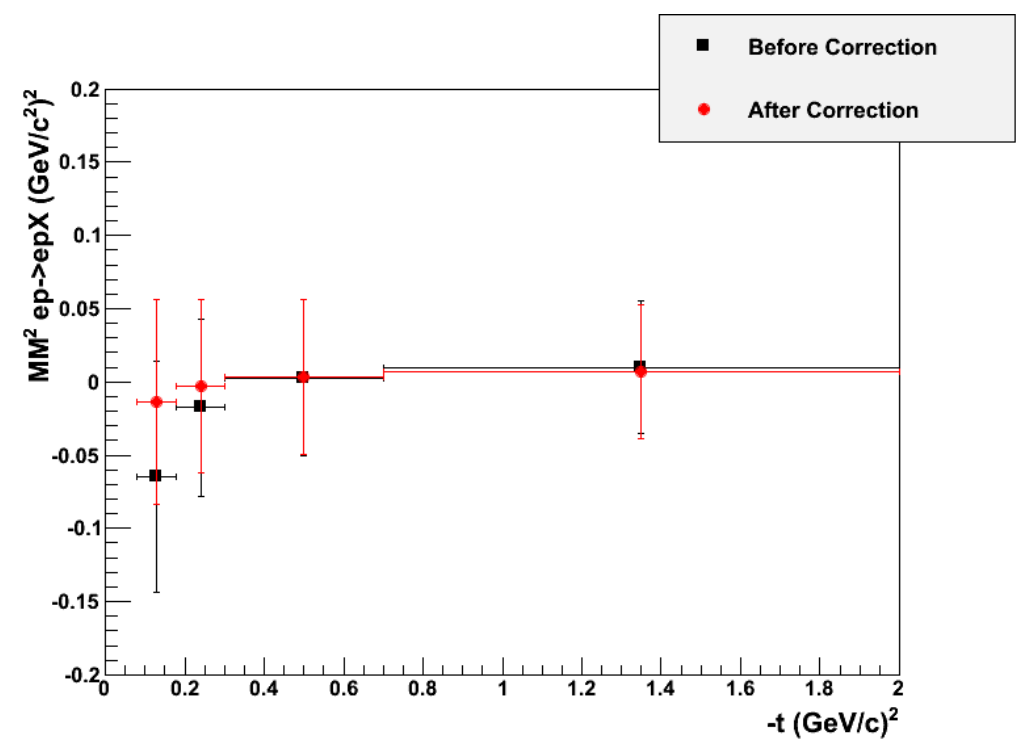

Fig. 4.10: Mean value of the missing mass of the ep system, for the experimental data (coming from Gaussian fits to the $M M^{2}(e p)$ distribution for each $t$-bin, produced with $\mathrm{NH}_{3}$ data as a function of $-t$, before (black) and after (red) energy-loss corrections. The horizontal error bars indicate the size of the - $t$ bin, the vertical error bars represent $\sigma$, the Gaussian width of the missing-mass peak. 


\subsection{Dilution Factor Correction}

As discussed in Section 4.2.2, to account for the nuclear background associated with the ${ }^{14} \mathrm{NH}_{3}$ target material, runs on a ${ }^{12} \mathrm{C}$ target were taken periodically throughout the experiment. To study the dilution factor for both the DVCS and $e p \pi^{0}$ channels, exclusivity cuts (Sections 3.5 and 3.6) were applied to both $\mathrm{C}_{12}$ and $\mathrm{NH}_{3}$ data, and the dilution factor determined for each channel using Equation 4.20.

$$
D_{f}=\frac{N_{N H_{3}}-c * N_{C}}{N_{N H_{3}}}
$$

Where $\mathrm{N}_{\mathrm{NH}_{3}}$, and $\mathrm{N}_{C}$ are the number of events normalized by their respective charge accumulation. The factor $c$, defined in Equation 4.21, was applied to $\mathrm{N}_{C}$ to take into account the different target lengths and materials for $\mathrm{NH}_{3}$ and $\mathrm{C}_{12}$ runs [49],

$$
c=\frac{\left(\left(L-l_{N H_{3}}\right) * \rho_{H e}\right)+\left(l_{N H_{3}} * \rho_{N H_{3}} * 14 / 17\right)+\left(l_{K} * \rho_{K}\right)+\left(l_{A l} * \rho_{A l}\right)}{\left(\left(L-l_{C}\right) * \rho_{H e}\right)+\left(l_{C} * \rho_{C}\right)+\left(l_{K} * \rho_{K}\right)+\left(l_{A l} * \rho_{A l}\right)} \simeq 0.7
$$

with:

- $L$, the total length between the entrance and exit windows in the target chamber

- $l_{N H_{3}}, l_{C}, l_{K}, l_{A l}$, the packing fraction, or effective length of the ammonia target, carbon target, Kapton target cell covers, and aluminum target chamber windows respectively

- $\rho_{H e}, \rho_{N H_{3}}, \rho_{C}, \rho_{K}, \rho_{A l}$, the volume densities of the liquid helium in the target chamber, ammonia target, carbon target, Kapton target cell covers, and aluminum target chamber windows respectively

The statistics acquired on carbon during the EG1-DVCS experiment were much smaller than those for the $\mathrm{NH}_{3}$ data, so it was not possible to perform the dilution factor analyses for each of the 4-dimensional bins discussed in Section 3.7. The dependence of the dilution factor on each of the 4 kinematic variables was checked by integrating over the others (Figures 4.11-4.14). Both DVCS and $e p \pi^{0}$ dilution factors show an approximately flat dependence in each of the 4 kinematic variables $\left(x_{B}\right.$ shows the stronger deviations from the constant behavior, but the linear fit is still valid), and the fit results don't vary within error bars. It can be safely assumed, therefore, a constant value of $D_{f}$ for all the kinematic bins, for both the DVCS and $e p \pi^{0}$ analysis. Table 4.9 lists the dilution values used. 


\begin{tabular}{|l|c|c|}
\hline Run Period & $D_{f}^{e p \gamma}$ & $D_{f}^{e p \pi^{0}}$ \\
\hline \hline A & $0.912 \pm 0.009$ & $0.921 \pm 0.016$ \\
B & $0.928 \pm 0.006$ & $0.896 \pm 0.010$ \\
\hline
\end{tabular}

Table 4.9: DVCS and $e p \pi^{0}$ dilution results with statistical error for EG1-DVCS Run Periods $\mathrm{A}$ and $\mathrm{B}$.
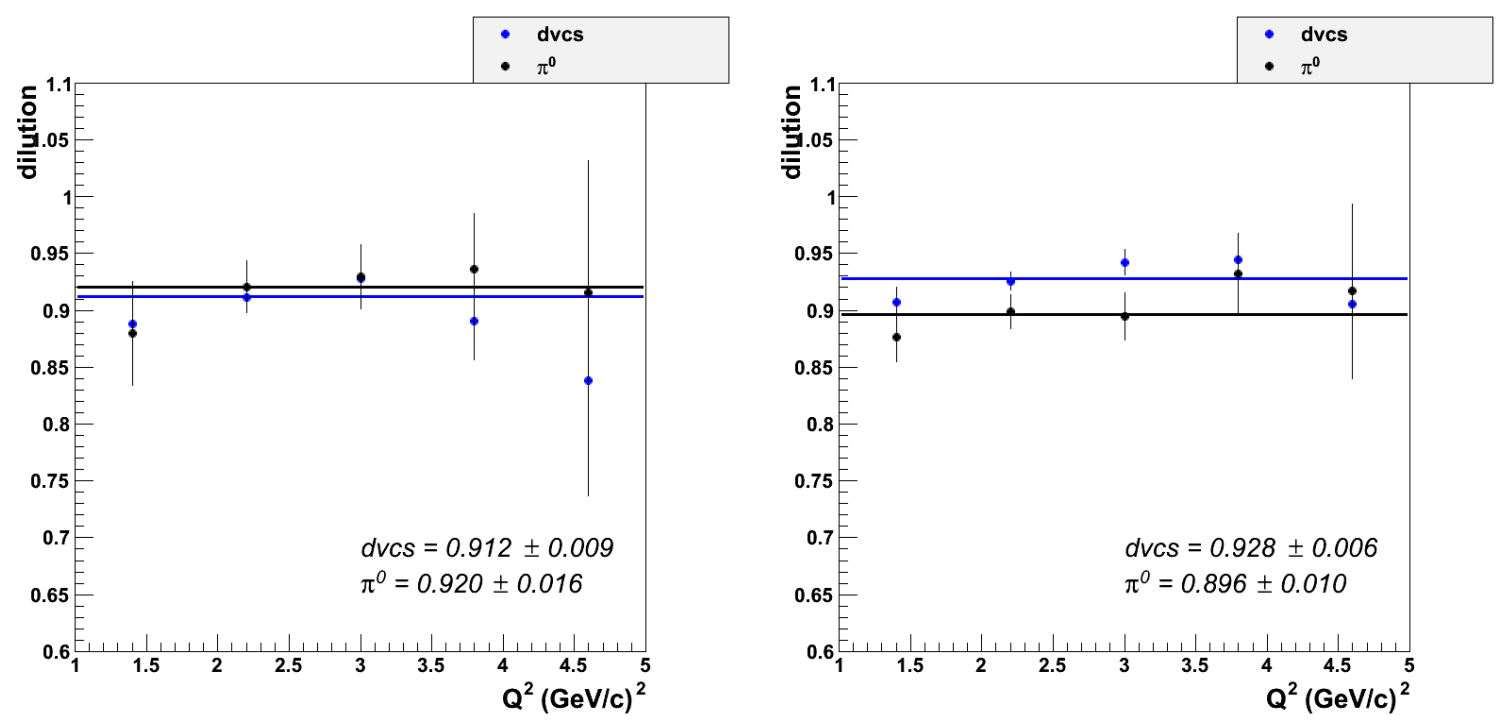

Fig. 4.11: Dilution factor, $D_{f}$, as a function of $Q^{2}$, for the DVCS analysis (blue points) and for the $e p \pi^{0}$ analysis (black points) for EG1-DVCS Run Period A (left) and Run Period B (right). 

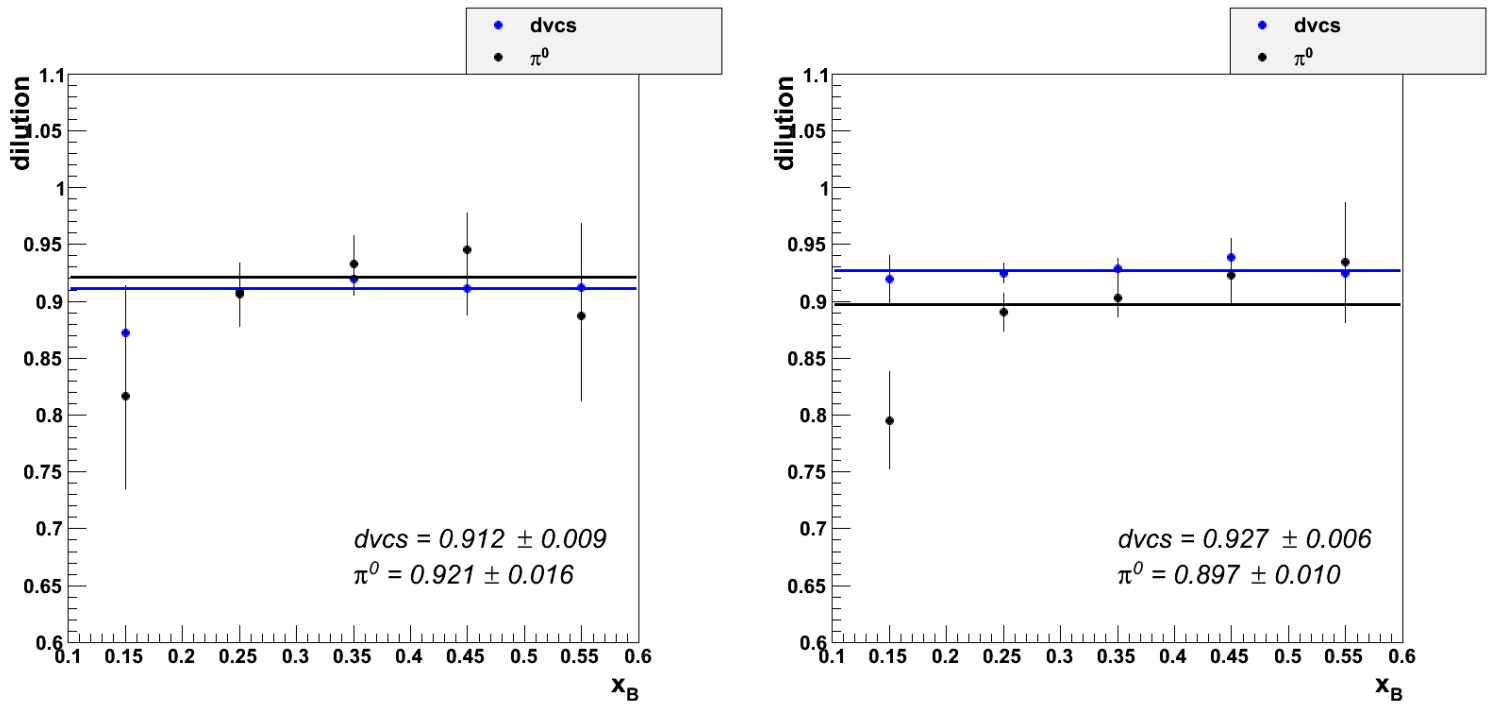

Fig. 4.12: Dilution factor, $D_{f}$, as a function of $x_{B}$, for the DVCS analysis (blue points) and for the $e p \pi^{0}$ analysis (black points) for EG1-DVCS Run Period A (left) and Run Period B (right).
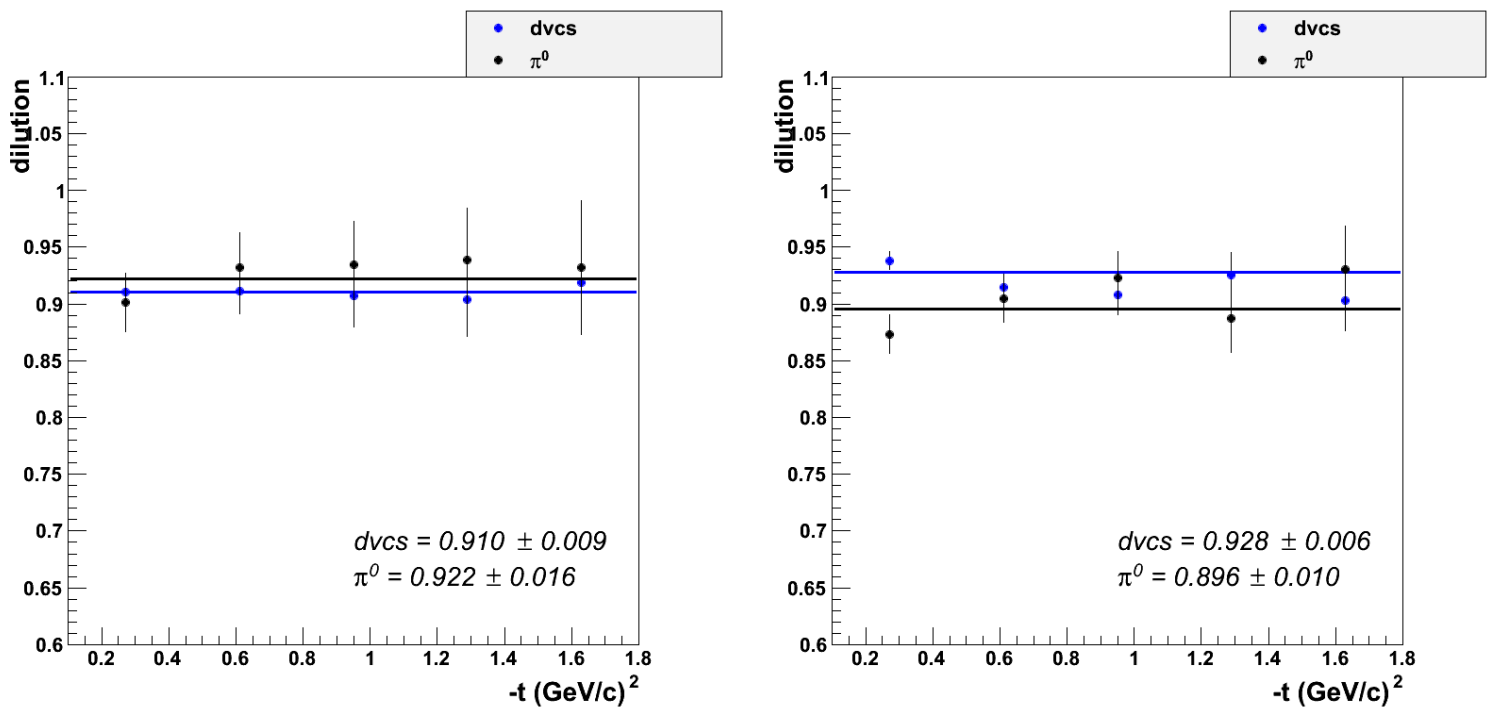

Fig. 4.13: Dilution factor, $D_{f}$, as a function of $-t$, for the DVCS analysis (blue points) and for the $e p \pi^{0}$ analysis (black points) for EG1-DVCS Run Period A (left) and Run Period B (right). 

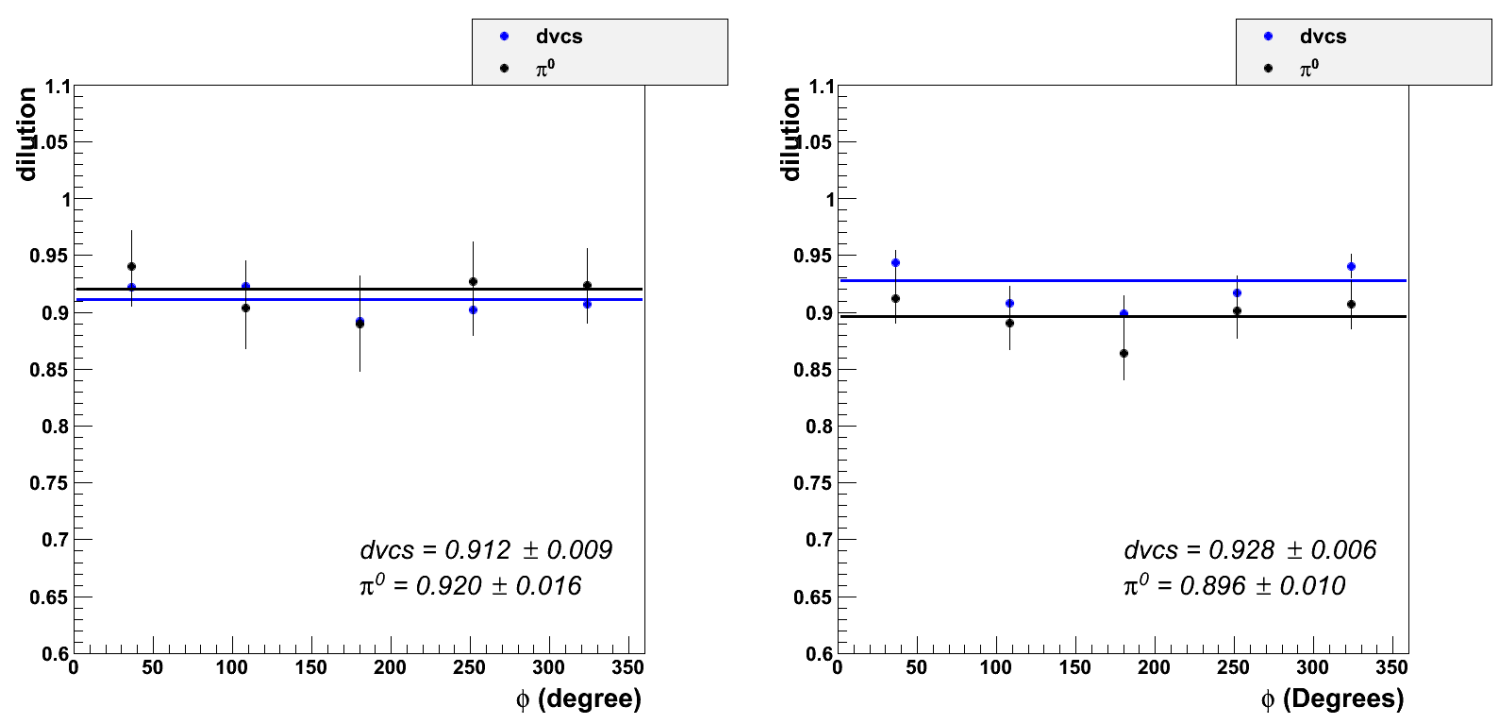

Fig. 4.14: Dilution factor, $D_{f}$, as a function of $\phi$, for the DVCS analysis (blue points) and for the $e p \pi^{0}$ analysis (black points) for EG1-DVCS Run Period A (left) and Run Period B (right).

\section{7 $\pi^{0}$ Contamination}

The number of detected DVCS events, $N_{D V C S}$, cannot be explicitly measured. There is contamination from $\pi^{0}$ events where only a single photon is detected, $N_{\pi^{0}(1 \gamma)}$. What can be measured is the sum of $N_{D V C S}$ and $N_{\pi^{0}(1 \gamma)}$. From Monte Carlo simulation, the fraction of single detected photon $\pi^{0}$ events in the detected single photon events, $B c k g r_{\pi^{0}}$ can be determined, and from that, the $N_{D V C S}$ can be calculated. The fraction, $B c k g r_{\pi^{0}}$ is defined as 4.22 with the unknown quantities in bold.

$$
B \operatorname{ckgr} r_{\pi^{0}}=\frac{\mathbf{N}_{\mathbf{d a t a}}^{\mathbf{e p} \mathbf{0}_{(\mathbf{1} \gamma)}}}{N_{\text {data }}^{e-\gamma}}
$$

where:

- $\mathbf{N}_{\mathbf{d a t a}}^{\mathbf{e p} \pi^{\mathbf{0}}(\mathbf{1} \gamma)}$ is the number of $e p \pi^{0}$ events where only a single photon is detected from data

- $N_{d a t a}^{e p \gamma}$ is the number of detected single photon events from data $N_{D V C S}+N_{e p \pi^{0}(1 \gamma)}$ To determine $\mathbf{N}_{\mathbf{d a t a}}^{\mathbf{e p} \pi^{\mathbf{0}}(\mathbf{1} \gamma)}$, one can use the ratios in Equations 4.23 and 4.24.

$$
R_{e p \pi^{0}(1 \gamma)}=\frac{N_{r e c}^{e p \pi^{0}(1 \gamma)}}{N_{g e n}^{e p \pi^{0}}}=\frac{\mathbf{N}_{\text {data }}^{\text {ep } \pi^{\mathbf{0}}(\mathbf{1} \gamma)}}{\mathbf{N}_{\text {events }}^{\text {ep } \pi^{0}}}
$$




$$
R_{e p \pi^{0}(2 \gamma)}=\frac{N_{r e c}^{e p \pi^{0}(2 \gamma)}}{N_{g e n}^{e p \pi \pi^{0}}}=\frac{N_{d a t a}^{e p \pi^{0}(2 \gamma)}}{\mathbf{N}_{\text {events }}^{\text {ep } \pi^{0}}}
$$

where:

- $N_{\text {gen }}^{e p \pi^{0}}$ are the number of generated $\pi^{0}$ electroproduction events

- $N_{r e c}^{i}$ are the number of GSIM reconstructed events for single $i=e p \pi^{0}(1 \gamma)$ and two $i=e p \pi^{0}(2 \gamma)$ photon $\pi^{0}$ electroproduction

- $N_{\text {data }}^{i}$ are the number of detected events from data for single $\mathbf{i}=\mathbf{e p} \pi^{\mathbf{0}}(\mathbf{1} \gamma)(\mathbf{u n k n o w n})$ and two $i=e p \pi^{0}(2 \gamma)$ photon $\pi^{0}$ electroproduction

- $\mathbf{N}_{\text {events }}^{\text {ep } \pi^{\mathbf{0}}}$ (unknown) are the number of actual events

Therefore, if the event selection cuts (Sections 3.5 and 3.6) are equivilent between reconstructed events and data, the fraction $B c k g r_{\pi^{0}}$ can equivocally be written as Equation 4.25.

$$
\begin{aligned}
\operatorname{Bckgr}_{\pi^{0}} & =\frac{R_{e p \pi^{0}(1 \gamma)}}{R_{e p \pi^{0}(2 \gamma)}} \frac{N_{\text {data }}^{e p \pi^{0}(2 \gamma)}}{N_{\text {data }}^{e p \gamma}} \\
& =R_{A c c}(1 \gamma / 2 \gamma) \frac{N_{\text {data }}^{e p \pi^{0}(2 \gamma)}}{N_{\text {data }}^{e p \gamma}}
\end{aligned}
$$

with:

$$
R_{A c c}(1 \gamma / 2 \gamma)=\frac{N_{r e c}^{e p \pi^{0}(1 \gamma)}}{N_{r e c}^{e p \pi^{0}(2 \gamma}}
$$

\subsubsection{Results for $\pi^{0}$ contamination}

The Monte-Carlo ratio $R_{A c c}(1 \gamma / 2 \gamma)$, defined in Equation 4.26, is shown in Figures 4.15 4.19 for each of the kinematic bins discussed in Section 3.7, as a function of $\phi$ for Run Period B (plots for Run Period A are shown in Appendix C). 

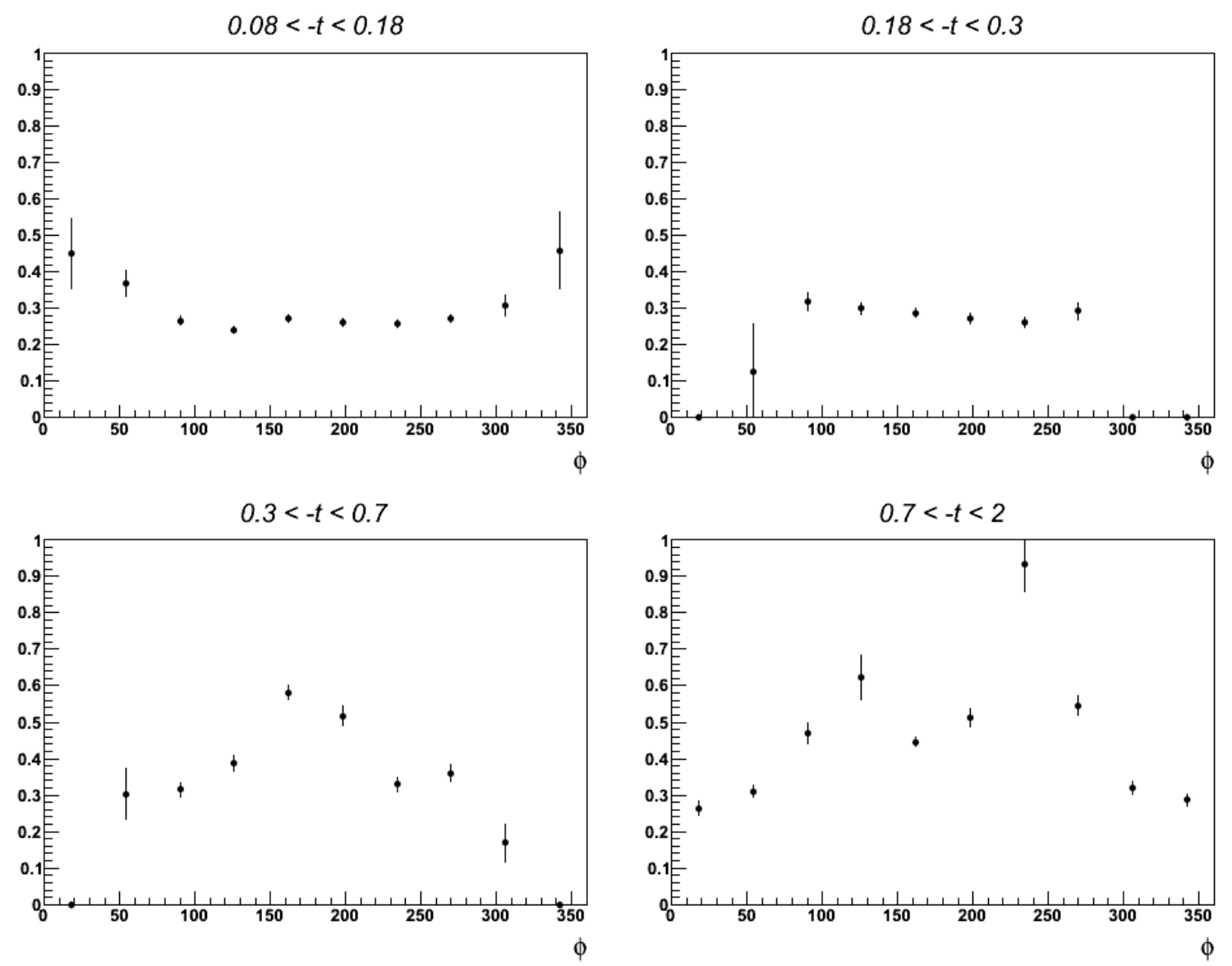

Fig. 4.15: Run Period B: Monte-Carlo ratio $R_{A c c}(1 \gamma / 2 \gamma)$, as a function of $\phi$, for each bin in $-t$, for the first bin in $Q^{2}-x_{B}$. 

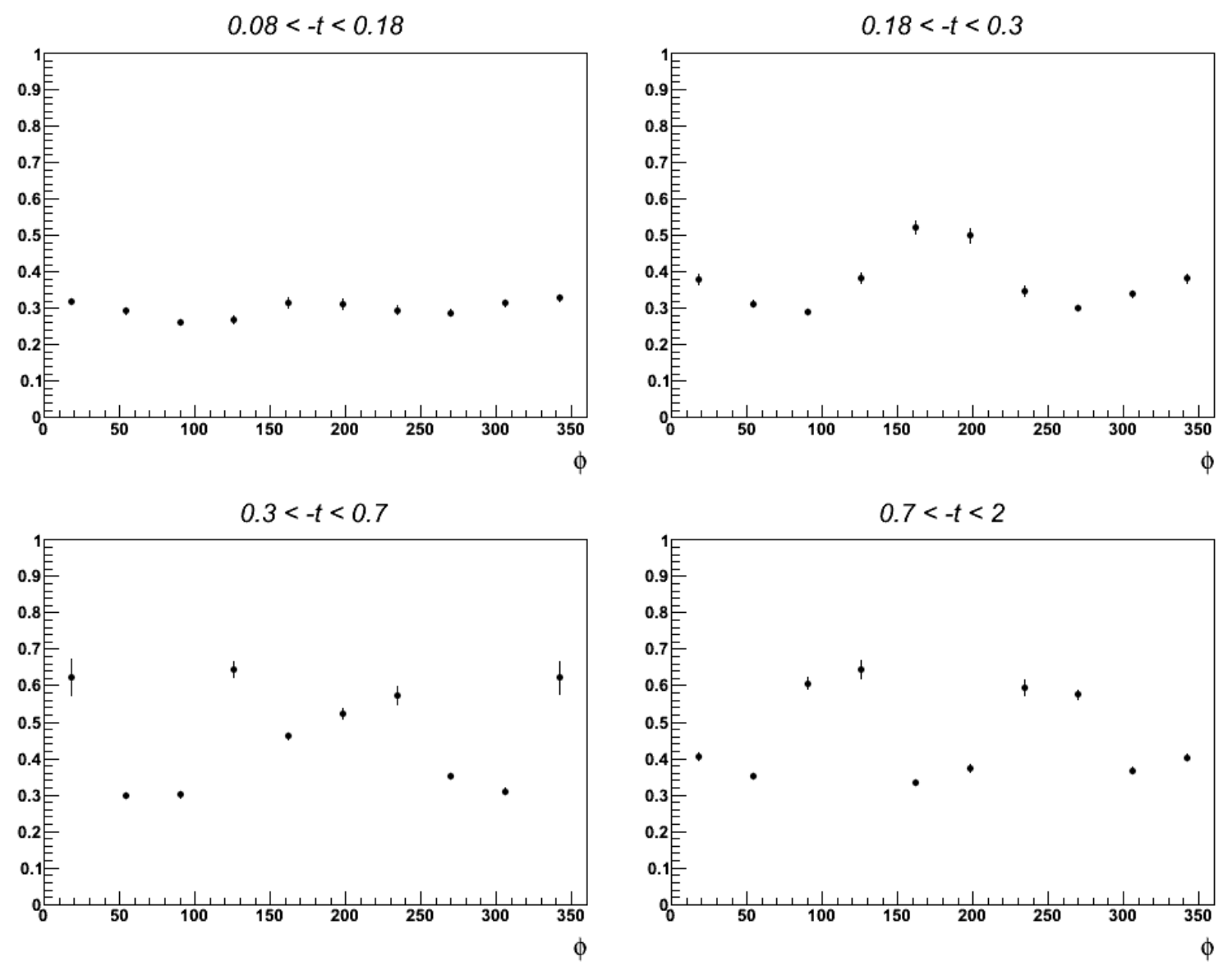

Fig. 4.16: Run Period B: Monte-Carlo ratio $R_{A c c}(1 \gamma / 2 \gamma)$, as a function of $\phi$, for each bin in $-t$, for the second bin in $Q^{2}-x_{B}$. 

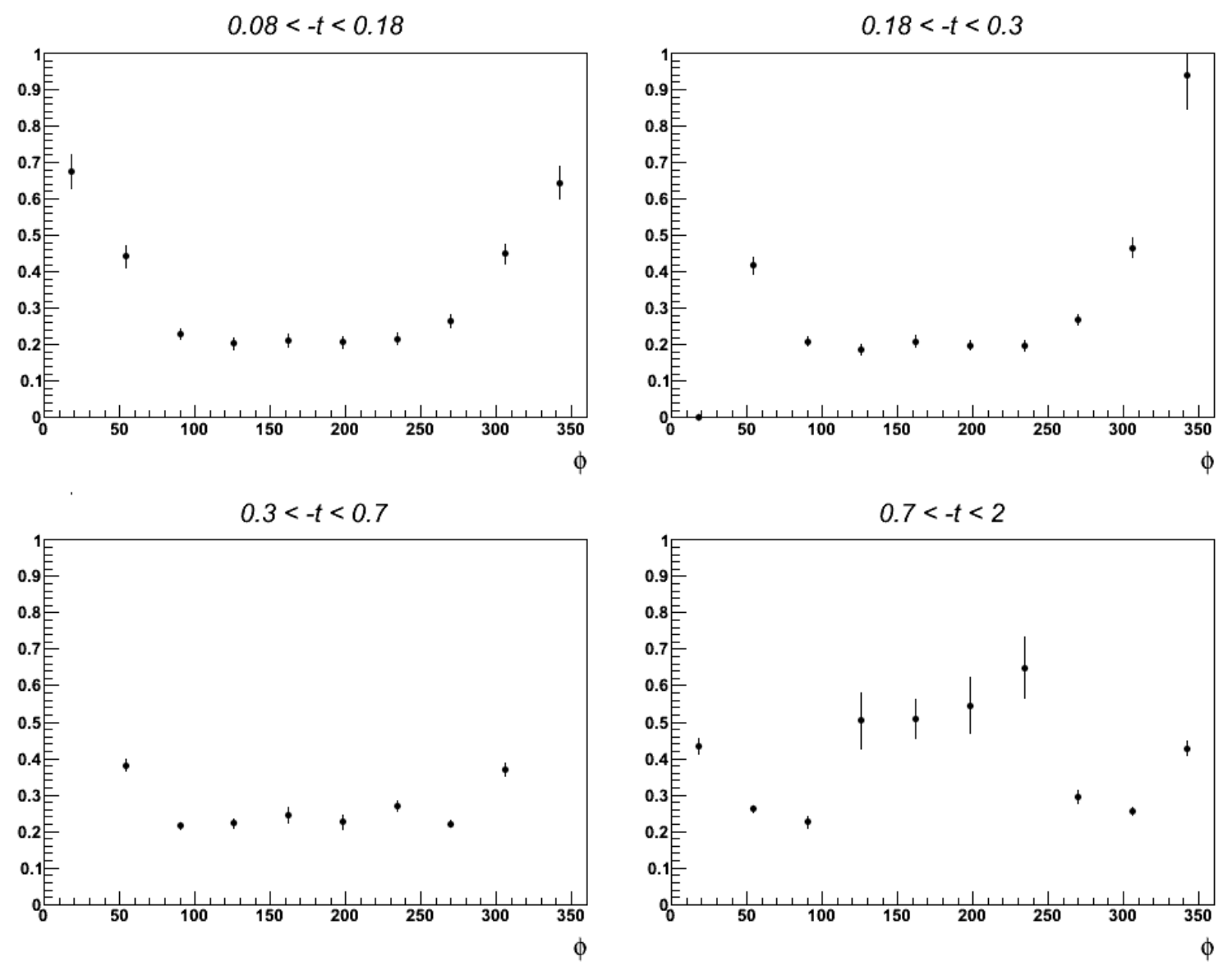

Fig. 4.17: Run Period B: Monte-Carlo ratio $R_{A c c}(1 \gamma / 2 \gamma)$, as a function of $\phi$, for each bin in $-t$, for the third bin in $Q^{2}-x_{B}$. 

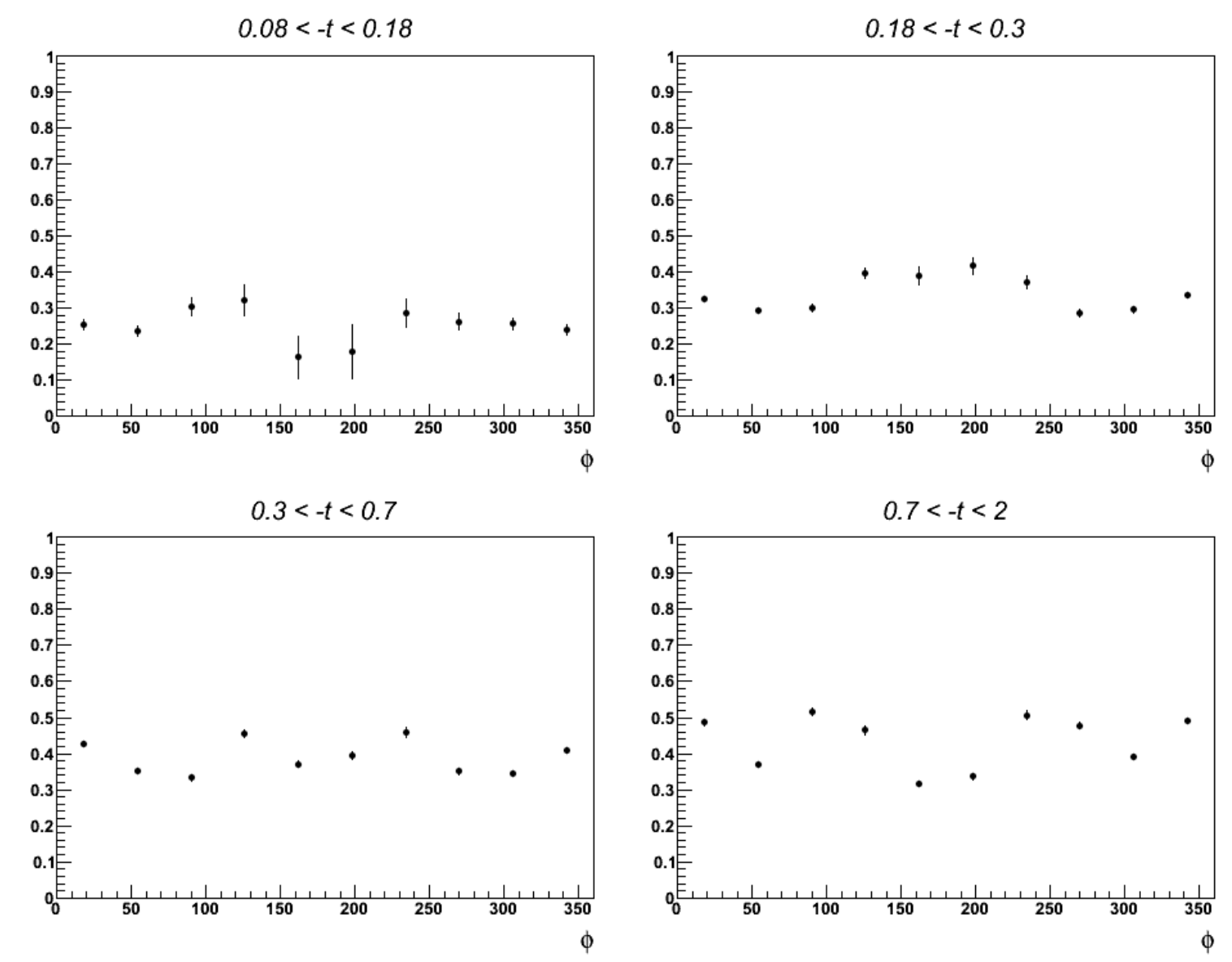

Fig. 4.18: Run Period B: Monte-Carlo ratio $R_{A c c}(1 \gamma / 2 \gamma)$, as a function of $\phi$, for each bin in $-t$, for the fourth bin in $Q^{2}-x_{B}$. 

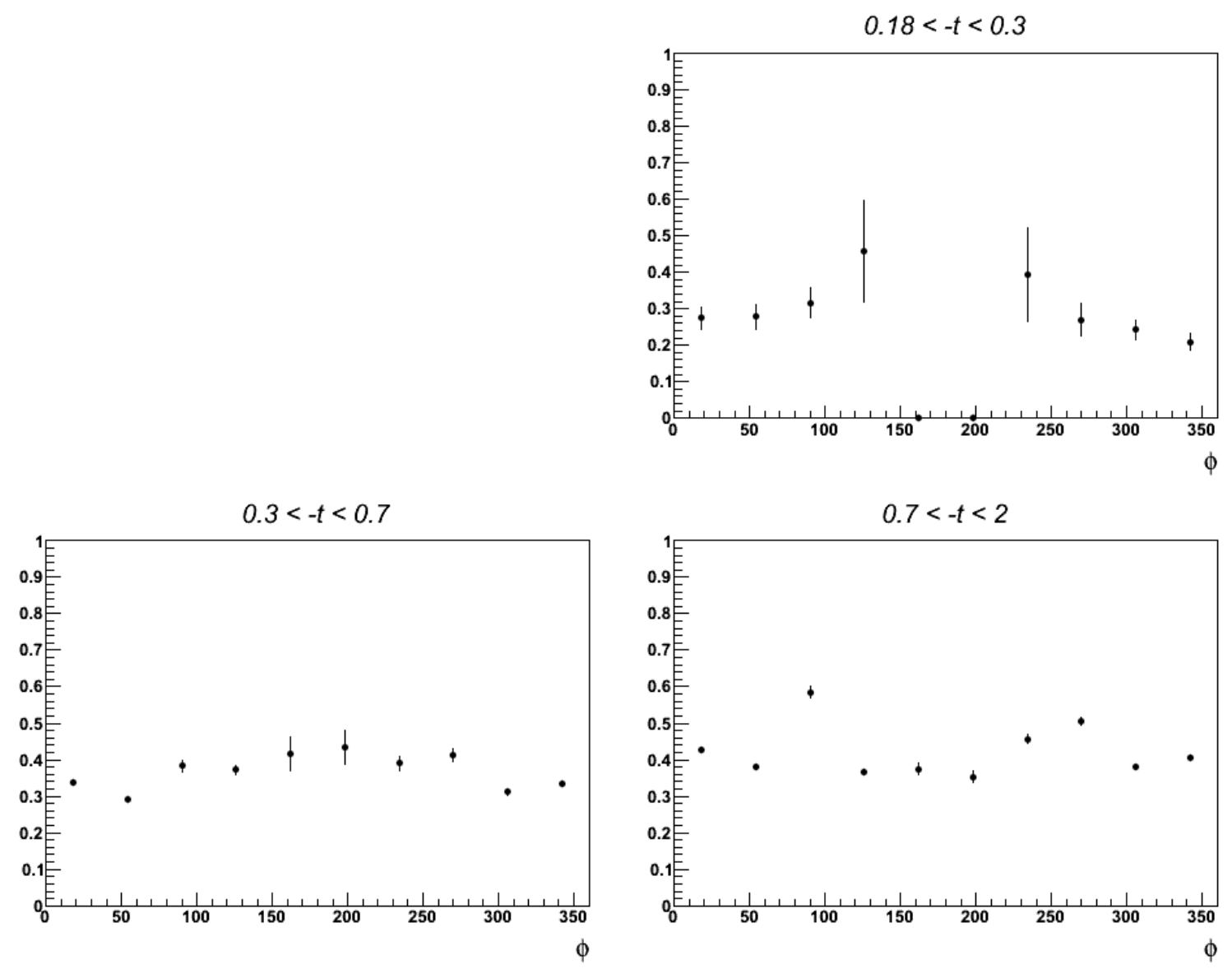

Fig. 4.19: Run Period B: Monte-Carlo ratio $R_{A c c}(1 \gamma / 2 \gamma)$, as a function of $\phi$, for each bin in $-t$, for the fifth bin in $Q^{2}-x_{B}$. 
Finally, the resulting relative background/signal ratios for $e p \pi^{0}(1 \gamma)$ events in the ep $\gamma$ sample, $B c k g r_{\pi^{0}}$, defined in Equation 4.25, are shown, as a function of the kinematics and of the target polarization sign, in Figures 4.20 - 4.24 for Run Period B (plots for Run Period A are shown in Appendix C). In absolute value, $B c k g r_{\pi^{0}}$ ranges from less than $5 \%$ (at low $-t$, low-mid $x_{B}$, high $Q^{2}$ ) to about $50 \%$, in the highest $-t$ bin, highest $x_{B}$, central $\phi$ range. However, what impacts the final asymmetries is not the size of the contamination itself, but the point-by-point difference of contamination for positive and negative target polarization.
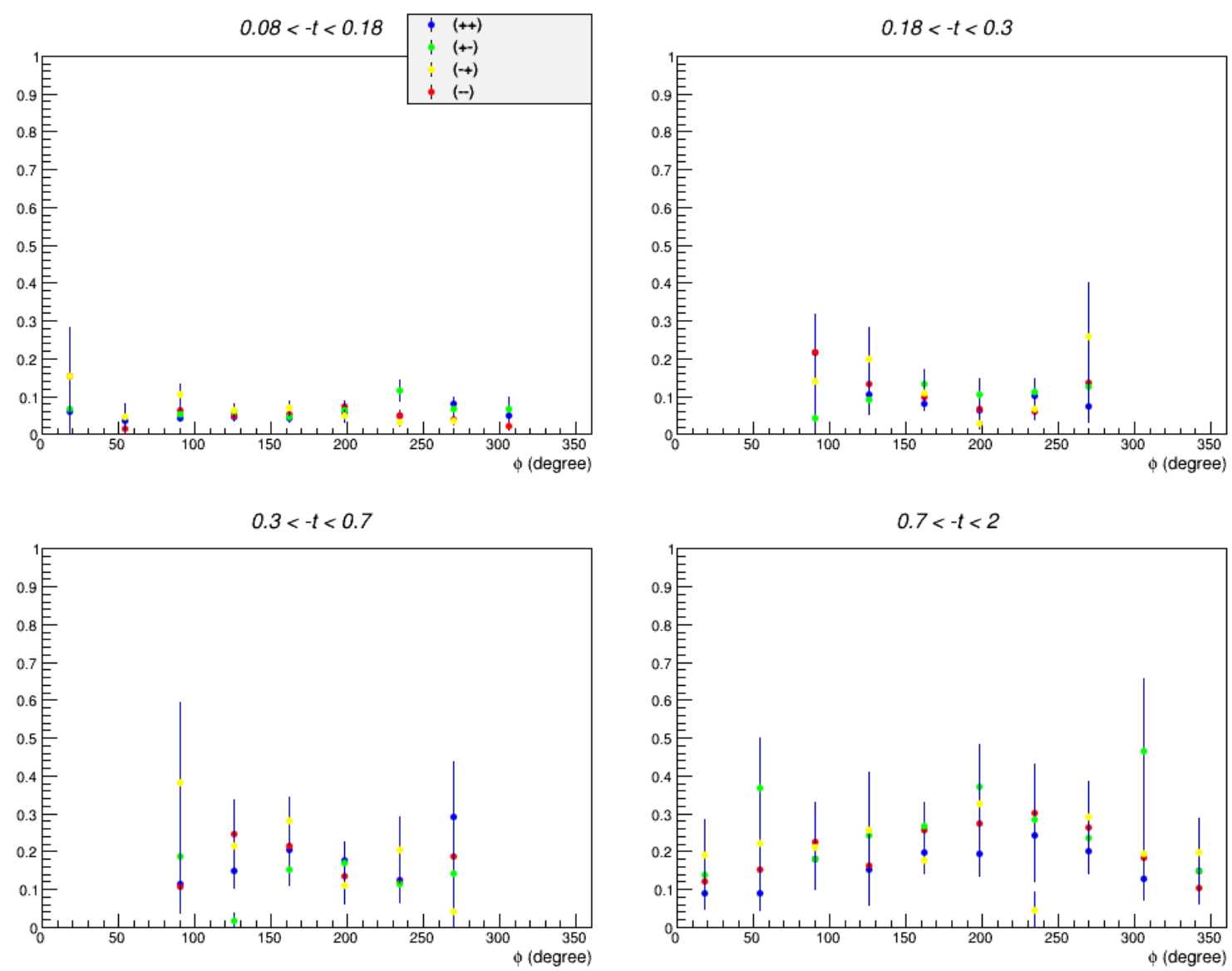

Fig. 4.20: Run Period B: Fraction of $\pi^{0}$ contamination over the total number of selected $e p \gamma$ events, $B c k g r_{\pi^{0}}$, as a function of $\phi$, for each bin in $-t$, for the first bin in $Q^{2}-x_{B}$. The color code, where the notation is $b t$, where $b$ is beam helicity, $t$ is target polarization sign, is: ++ blue, -+ red, +- green, -+ yellow. 

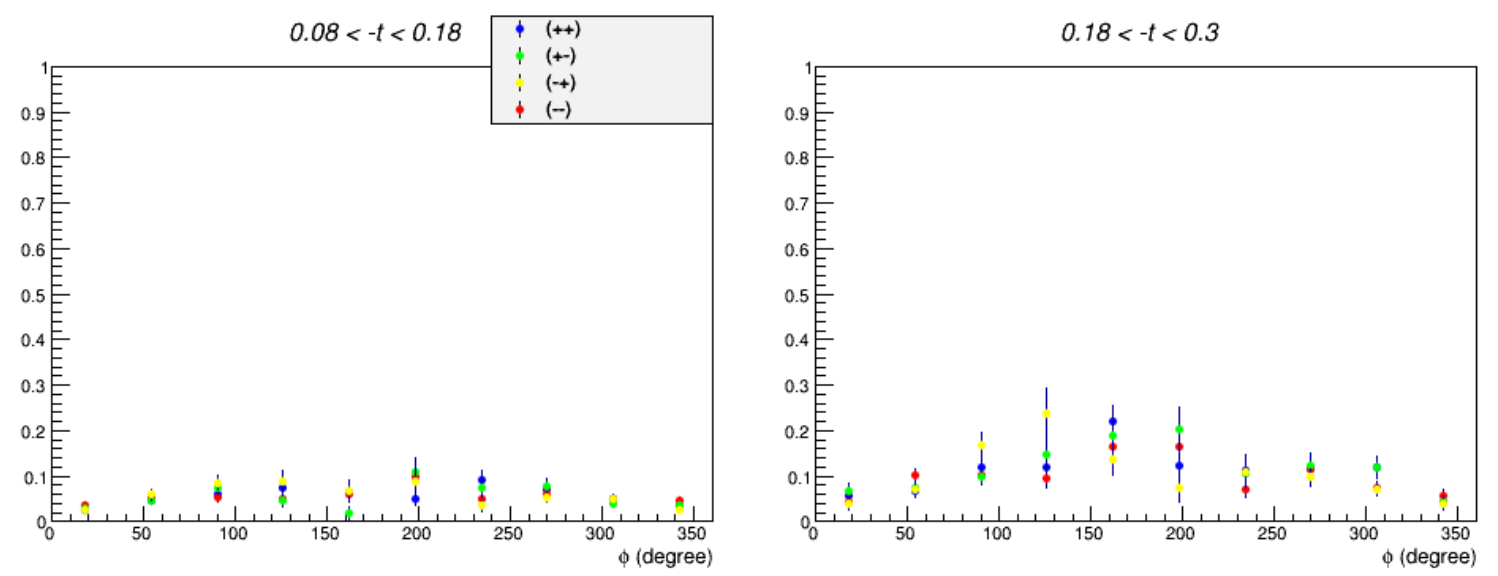

$0.3<-t<0.7$
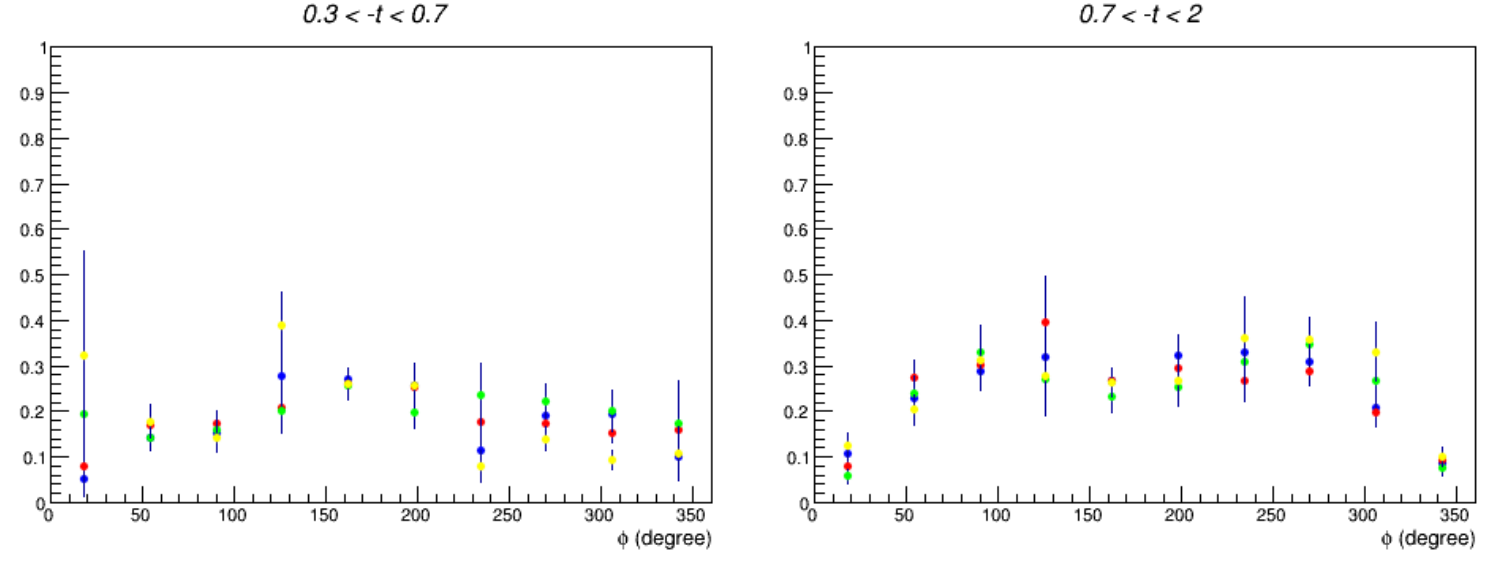

Fig. 4.21: Run Period B: Fraction of $\pi^{0}$ contamination over the total number of selected ep $\gamma$ events, $B c k g r_{\pi^{0}}$, as a function of $\phi$, for each bin in $-t$, for the second bin in $Q^{2}-x_{B}$. The color code, where the notation is $b t$, where $b$ is beam helicity, $t$ is target polarization sign, is: ++ blue, -+ red, +- green, -+ yellow. 

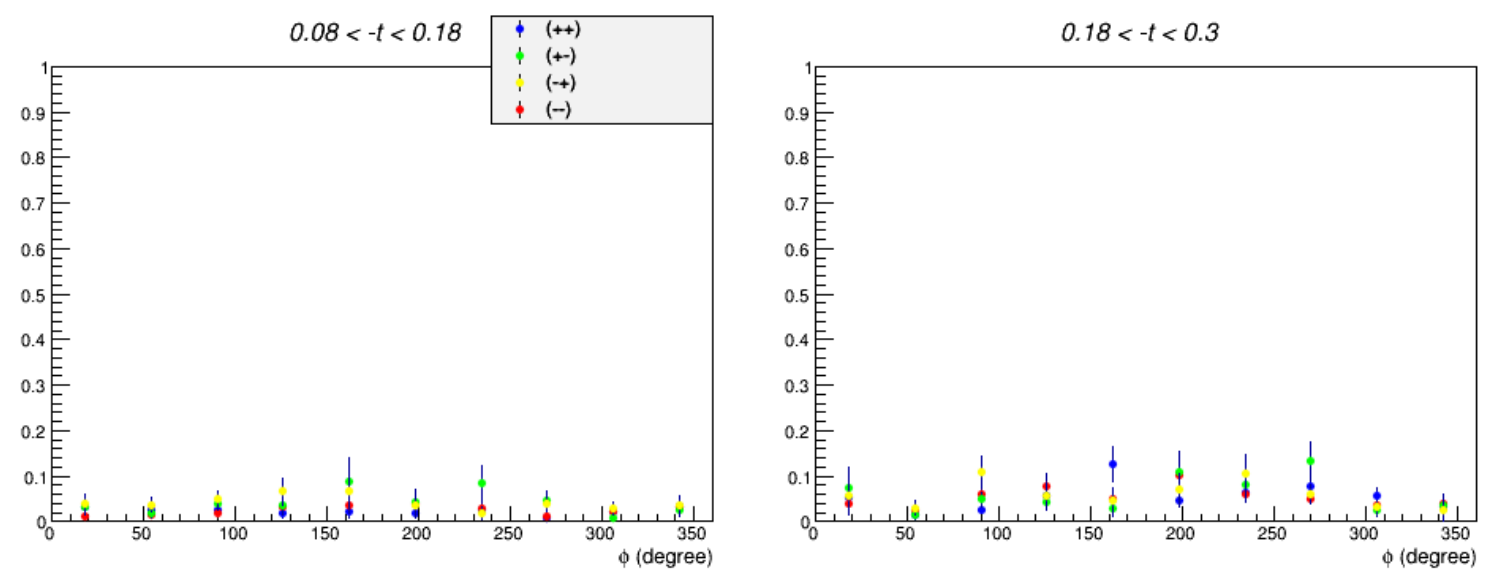

$0.3<-t<0.7$
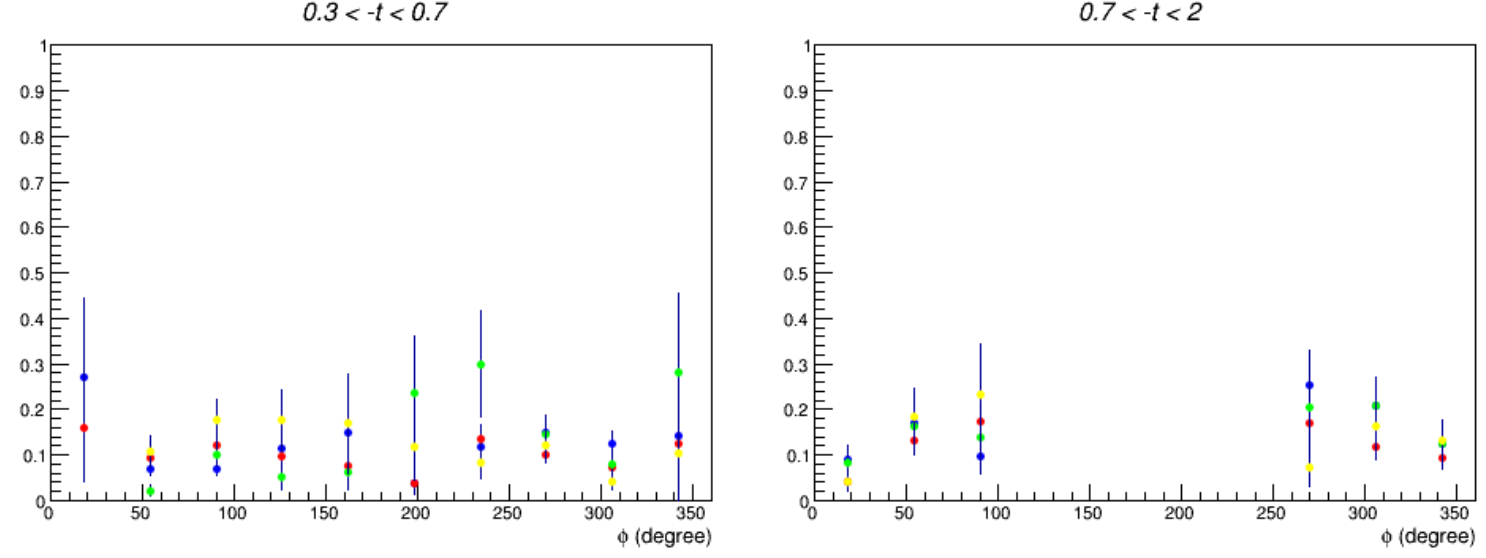

Fig. 4.22: Run Period B: Fraction of $\pi^{0}$ contamination over the total number of selected $e p \gamma$ events, $B c k g r_{\pi^{0}}$, as a function of $\phi$, for each bin in $-t$, for the third bin in $Q^{2}-x_{B}$. The color code, where the notation is $b t$, where $b$ is beam helicity, $t$ is target polarization sign, is: ++ blue, -+ red, +- green, -+ yellow. 

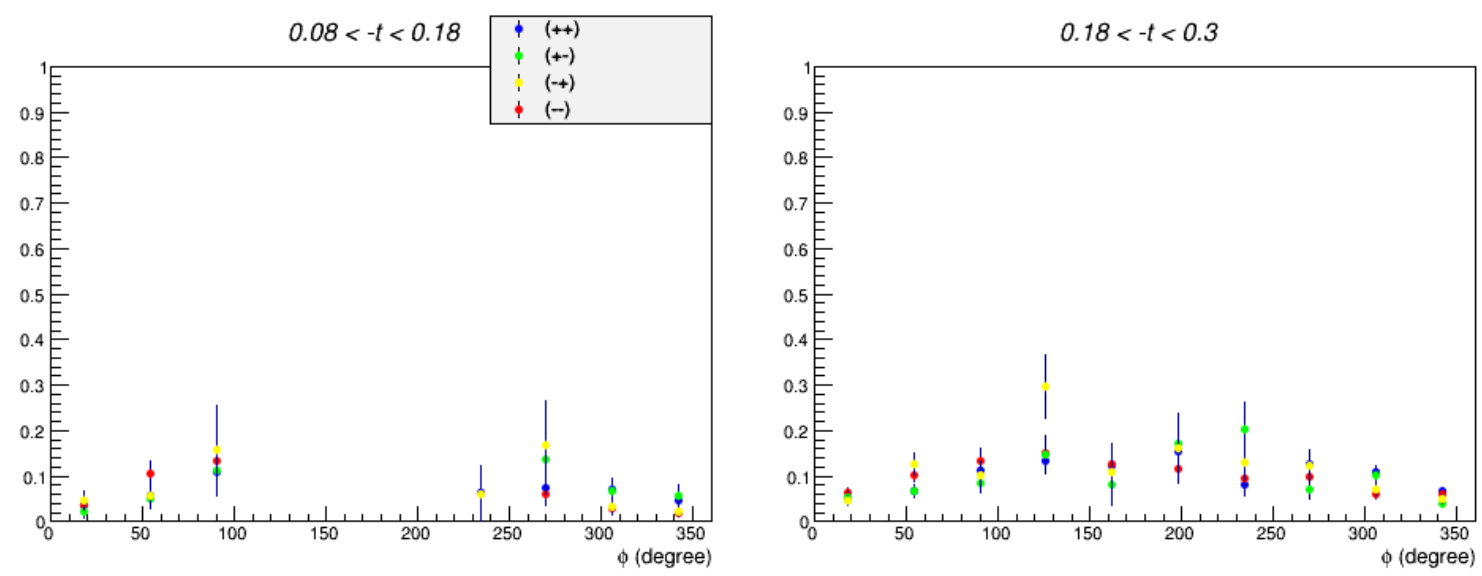

$0.3<-t<0.7$
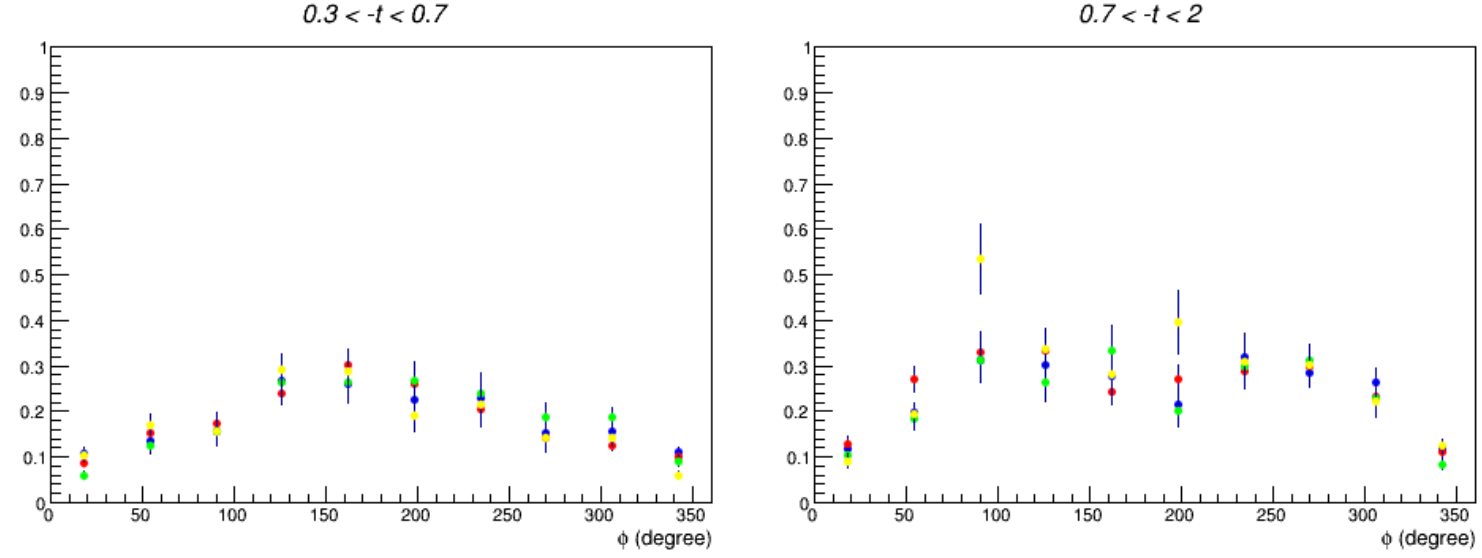

Fig. 4.23: Run Period B: Fraction of $\pi^{0}$ contamination over the total number of selected $e p \gamma$ events, $B c k g r_{\pi^{0}}$, as a function of $\phi$, for each bin in $-t$, for the fourth bin in $Q^{2}-x_{B}$. The color code, where the notation is $b t$, where $b$ is beam helicity, $t$ is target polarization sign, is: ++ blue, -+ red, +- green, -+ yellow. 

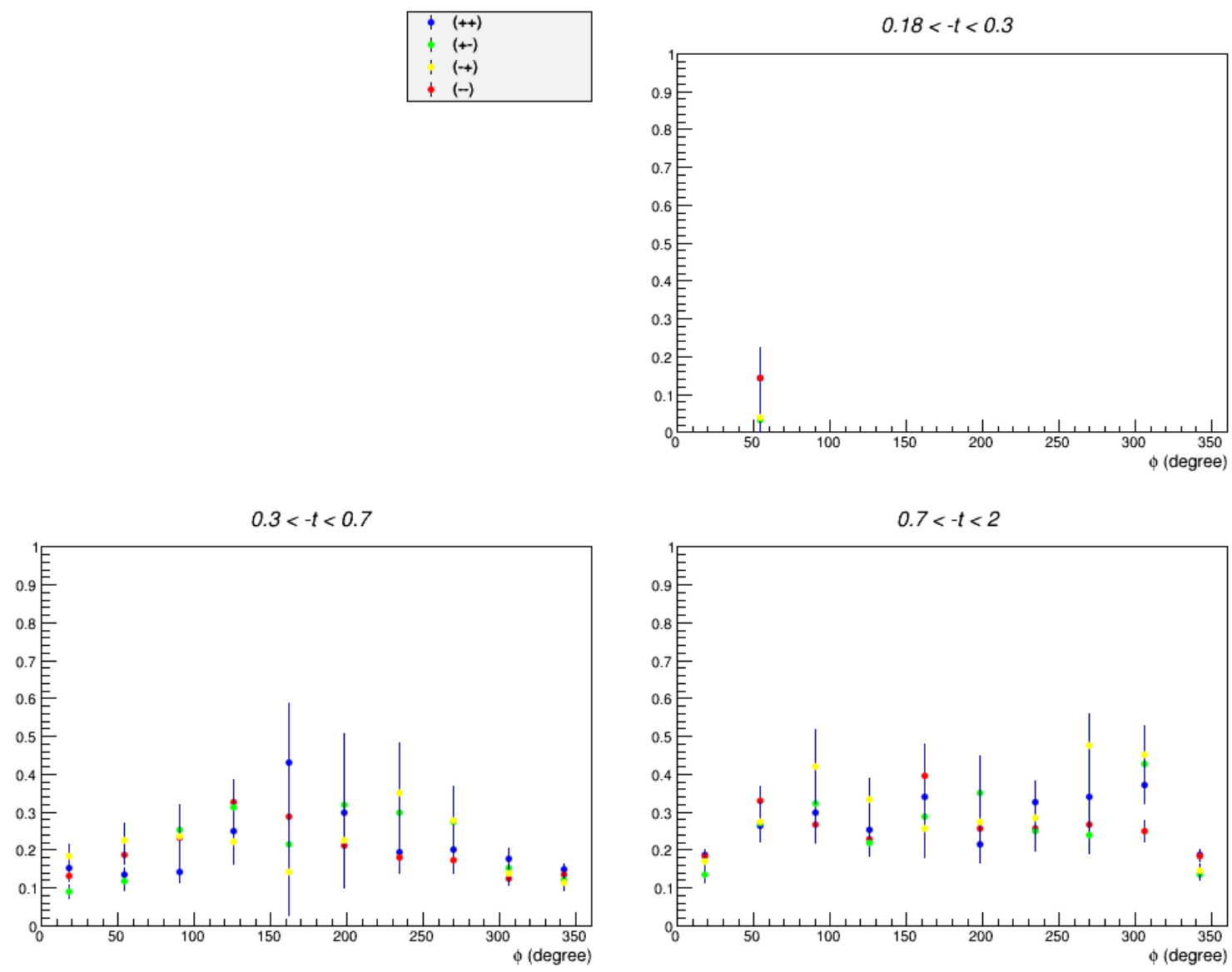

Fig. 4.24: Run Period B: Fraction of $\pi^{0}$ contamination over the total number of selected ep $\gamma$ events, $B c k g r_{\pi^{0}}$, as a function of $\phi$, for each bin in $-t$, for the fifth bin in $Q^{2}-x_{B}$. The color code, where the notation is $b t$, where $b$ is beam helicity, $t$ is target polarization sign, is: ++ blue, -+ red, +- green, -+ yellow 


\subsubsection{Effect of $\pi^{0}$ contamination}

The comparison between the target-spin asymmetries before and after subtraction of the $\pi^{0}$ contamination is shown for EG1-DVCS Run Period B data in Figures 4.25 - 4.29 for Run Period B (plots for Run Period A are shown in Appendix C). Comparable effects on the Run Period A data were observed.
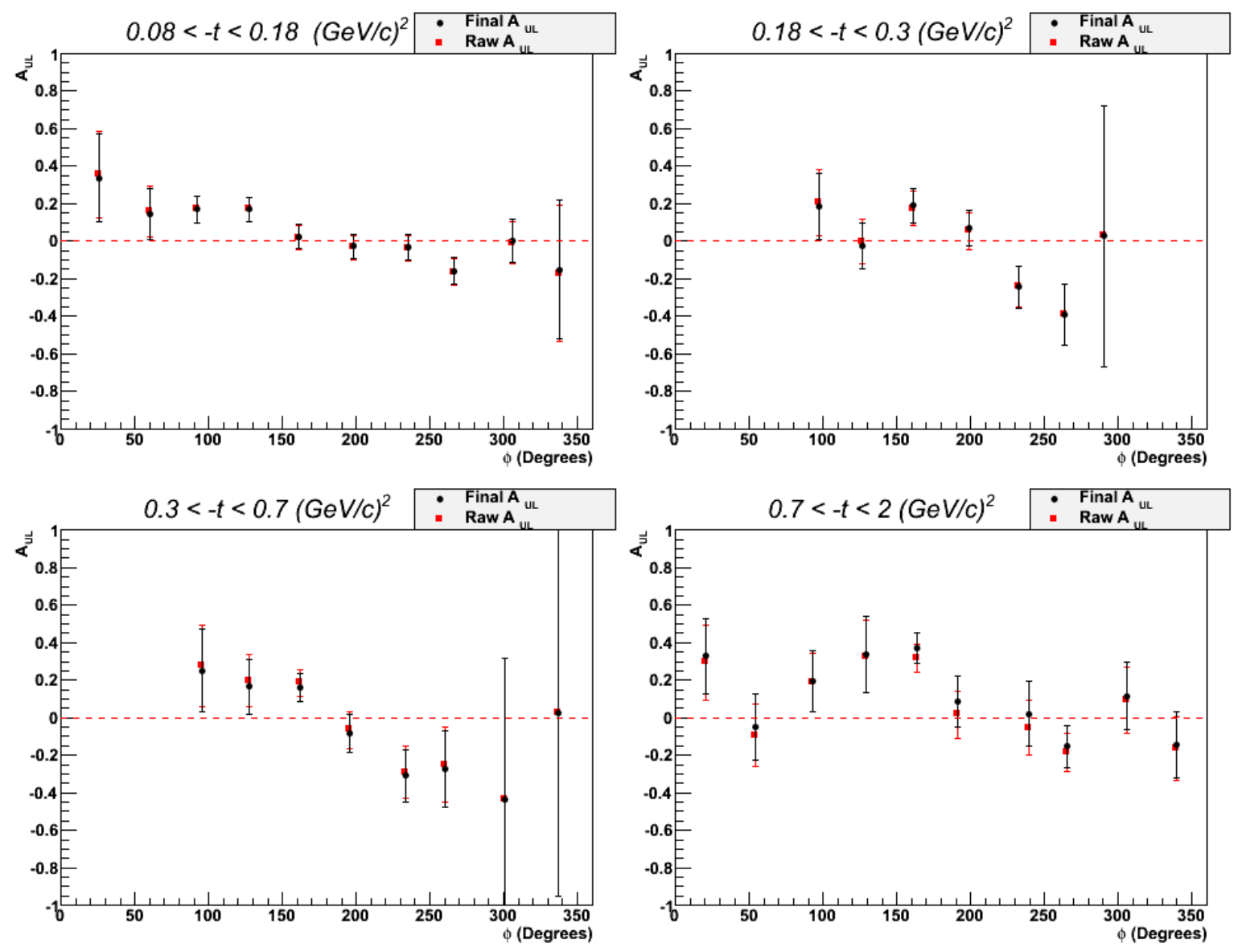

Fig. 4.25: Target-spin asymmetry Run Period B, as a function of $\phi$, for each bin in $-t$, and for the first bin in $Q^{2}-x_{B}$ shown for Part B data. The black and red points represent, respectively, the asymmetries including $\pi^{0}$ background subtraction and the raw asymmetries. 

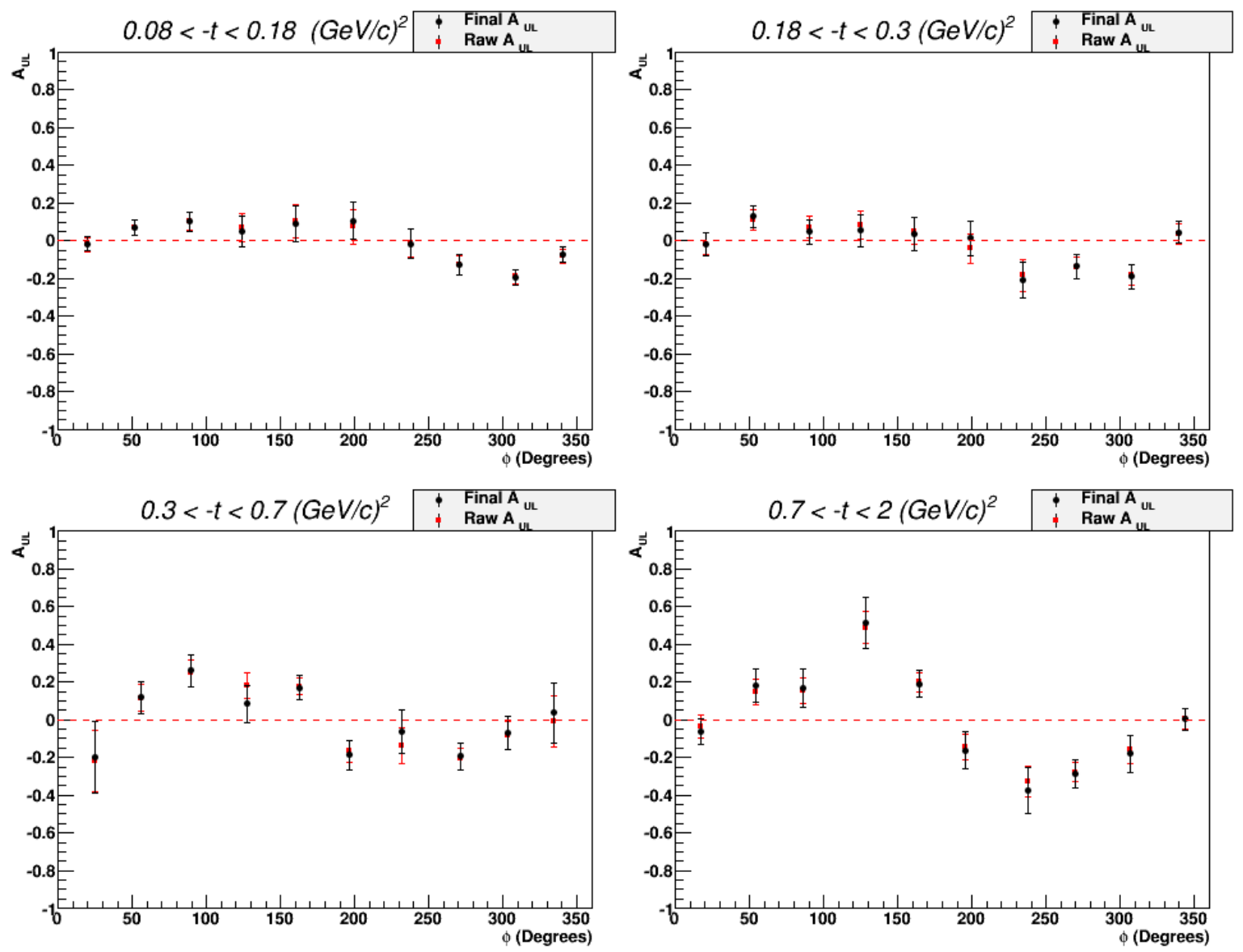

Fig. 4.26: Target-spin asymmetry Run Period B, as a function of $\phi$, for each bin in $-t$, and for the second bin in $Q^{2}-x_{B}$ shown for Part B data. The black and red points represent, respectively, the asymmetries including $\pi^{0}$ background subtraction and the raw asymmetries. 

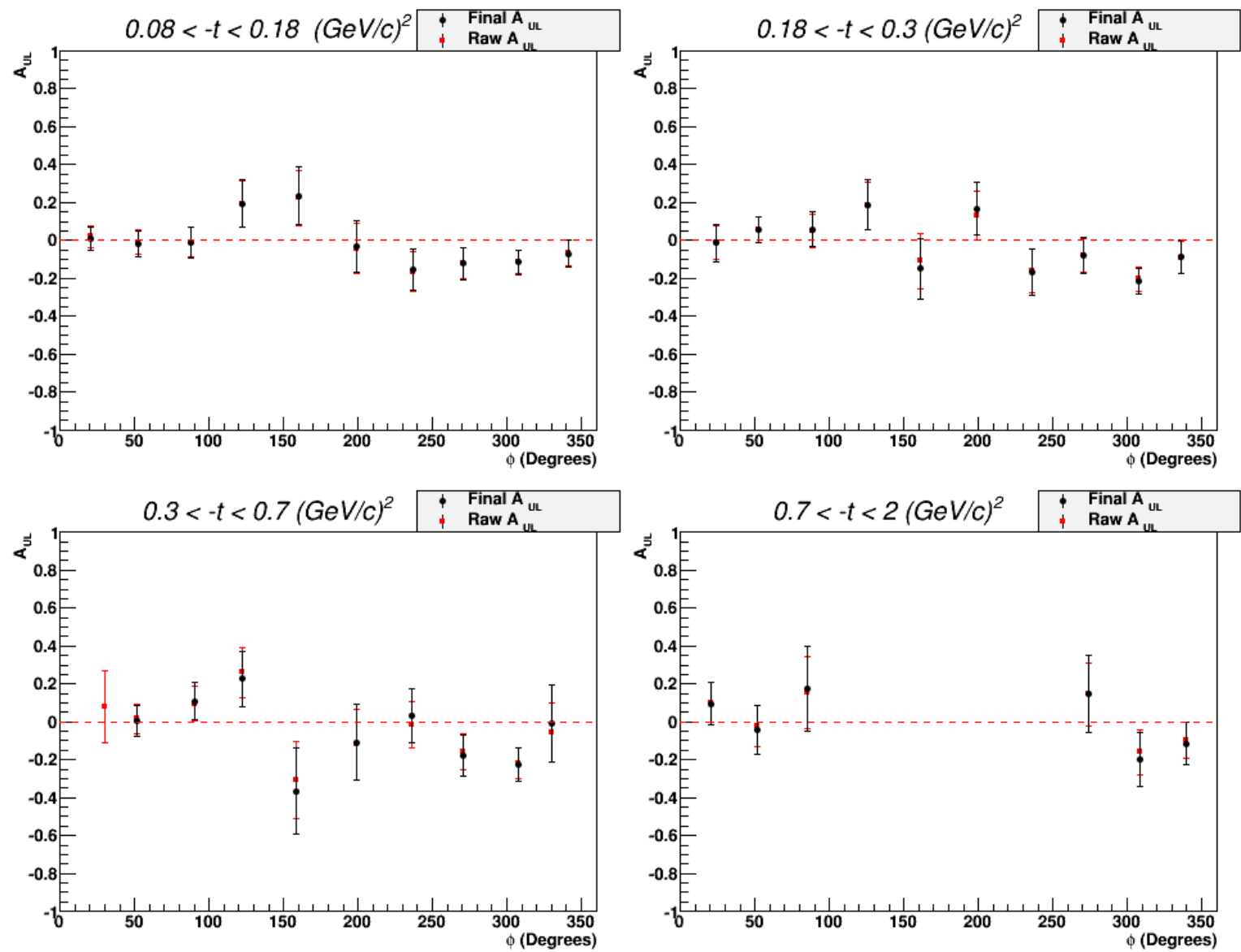

Fig. 4.27: Target-spin asymmetry Run Period B, as a function of $\phi$, for each bin in $-t$, and for the third bin in $Q^{2}-x_{B}$ shown for Part B data. The black and red points represent, respectively, the asymmetries including $\pi^{0}$ background subtraction and the raw asymmetries. 

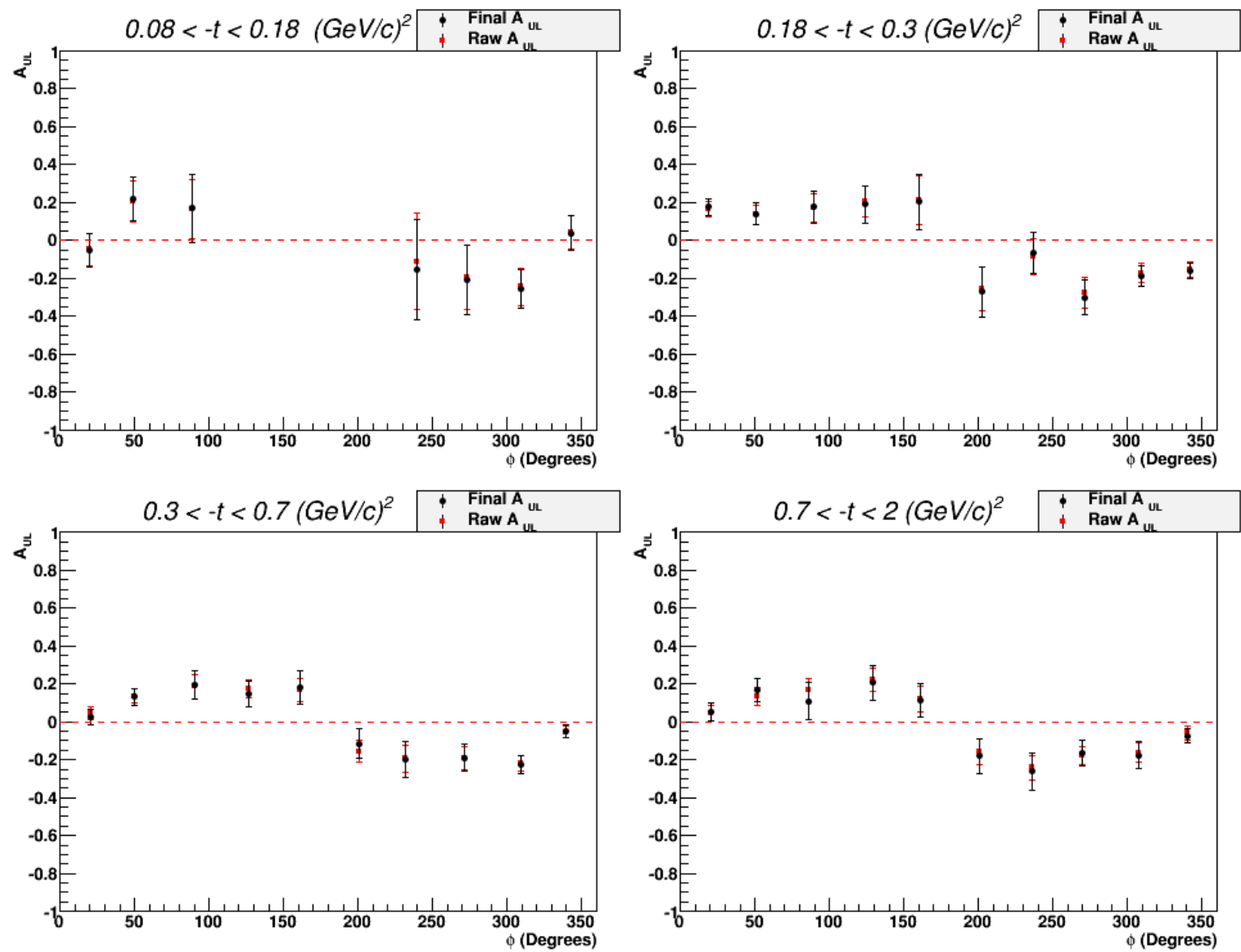

Fig. 4.28: Target-spin asymmetry Run Period B, as a function of $\phi$, for each bin in $-t$, and for the fourth bin in $Q^{2}-x_{B}$ shown for Part B data. The black and red points represent, respectively, the asymmetries including $\pi^{0}$ background subtraction and the raw asymmetries. 

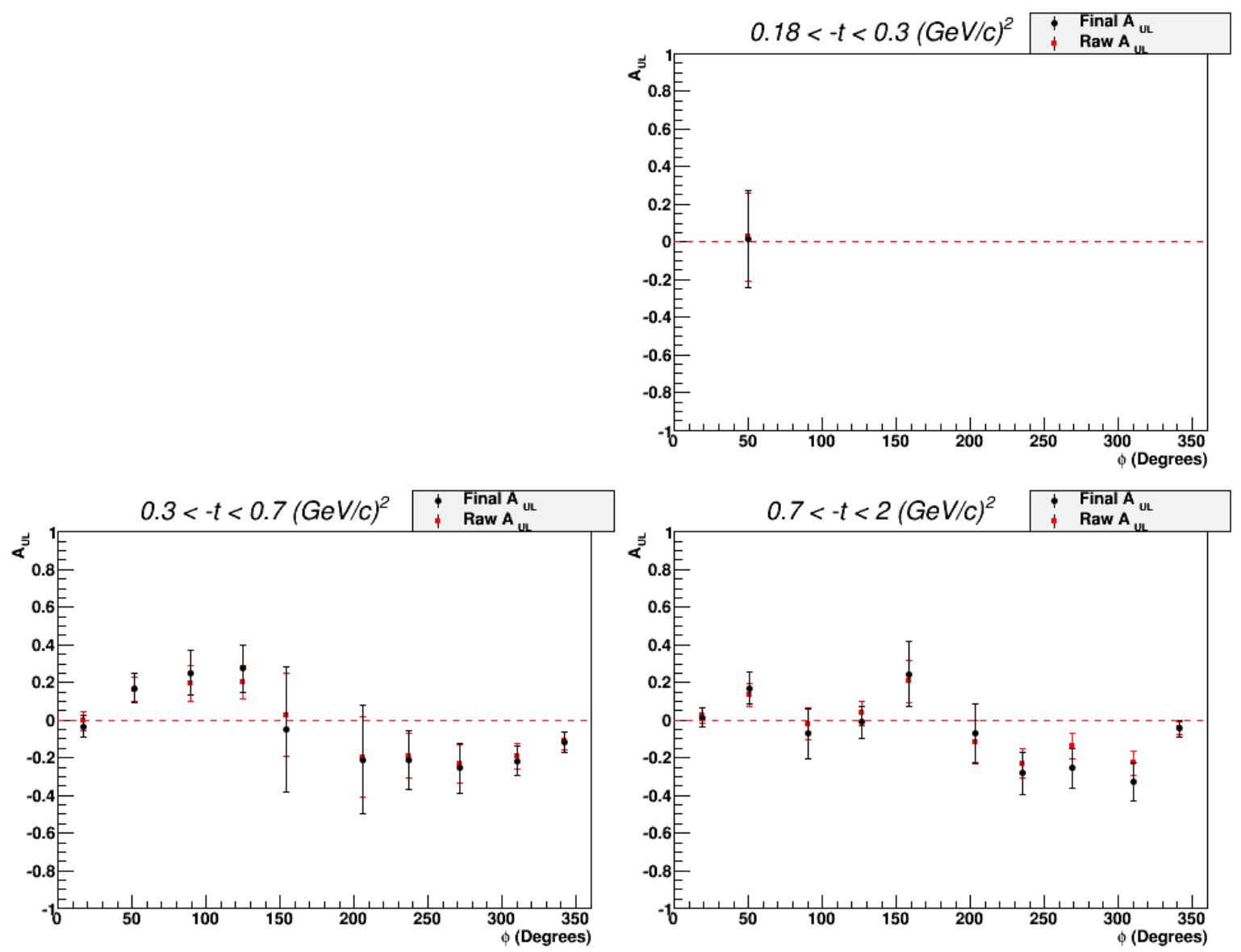

Fig. 4.29: Target-spin asymmetry Run Period B, as a function of $\phi$, for each bin in $-t$, and for the fifth bin in $Q^{2}-x_{B}$ shown for Part B data. The black and red points represent, respectively, the asymmetries including $\pi^{0}$ background subtraction and the raw asymmetries. 


\section{Chapter 5}

\section{Results}

\subsection{Target-Spin Asymmetry Definition}

The definition of the target-spin asymmetry, as discussed in Section 1.2, can be expressed as:

$$
A_{U L}=\frac{1}{D_{f}} \frac{\left(N^{\downarrow \Uparrow}+N^{\uparrow \Uparrow}\right)-\left(N^{\downarrow \Downarrow}+N^{\uparrow \Downarrow}\right)}{\left(N^{\downarrow \Uparrow}+N^{\uparrow \Uparrow}\right) P_{t}^{\Downarrow}+\left(N^{\downarrow \Downarrow}+N^{\uparrow \Downarrow}\right) P_{t}^{\Uparrow}}
$$

where $D_{f}$ is the dilution factor (Section 4.6), $P_{t}^{T}$ the proton target polarization, $T=\Uparrow / \Downarrow$ (Section 4.2.5), and the number of counts, $N^{B T}$, are defined as:

$$
N^{B T}=\left(1-B c k g r_{\pi^{0}}^{B T}\right) \frac{N_{e p \gamma}^{B T}}{F C^{B T}}
$$

where $N_{e p \gamma}^{B T}$ are the number of events passing the exclusivity cuts discussed in Section 3.5 with electron beam polarization, $B=\uparrow / \downarrow$, and target polarization, $T=\Uparrow / \Downarrow$. To normalize the data, the target polarization event counts are divided by the respective total accumulated charge, $F C^{B T}$. The $\pi^{0}$ background factor, $B \operatorname{ckgr} r_{\pi^{0}}^{B T}$, is defined as:

$$
B c k g r_{\pi^{0}}^{B T}=R_{A c c}(1 \gamma / 2 \gamma) \frac{N_{e p \pi^{0}}^{B T}}{N_{e p \gamma}^{B T}}
$$

therefore $N^{B T}$ can be expressed as:

$$
N^{B T}=\left(N_{e p \gamma}^{B T}-\frac{D_{f}^{e p \pi^{0}}}{D_{f}^{e p \gamma}} N_{e p \pi^{0}}^{B T} R_{A c c}(1 \gamma / 2 \gamma)\right) \frac{1}{F C^{B T}}
$$

The values of target polarization, $P_{t}^{\Downarrow}$ and $P_{t}^{\Uparrow}$, are the averages of the projection of target polarization on the virtual-photon direction, whose values are given in Table 4.6. 


\subsection{Statistical Error}

The statistical error, for each 4-dimensional bin, is defined in the following way:

$$
\delta A_{U L}=\sqrt{\left(\frac{\partial A_{U L}}{\partial N^{\uparrow \Uparrow}} \delta N^{\uparrow \Uparrow}\right)^{2}+\left(\frac{\partial A_{U L}}{\partial N^{\downarrow \Downarrow}} \delta N^{\downarrow \Downarrow}\right)^{2}+\left(\frac{\partial A_{U L}}{\partial N^{\uparrow \Downarrow}} \delta N^{\uparrow \Downarrow}\right)^{2}+\left(\frac{\partial A_{U L}}{\partial N^{\downarrow \Uparrow}} \delta N^{\downarrow \Uparrow \Uparrow}\right)^{2}}
$$

with:

$$
\begin{gathered}
\delta N^{B T}=\frac{1}{F C^{B T}} \sqrt{\left(\delta N_{e p \gamma}^{B T}\right)^{2}+\left(\delta R_{A c c}(1 \gamma / 2 \gamma) N_{e p \pi^{0}}^{B T} \frac{D_{f}^{e p \pi^{0}}}{D_{f}^{e p \gamma}}\right)^{2}+\left(\delta N_{e p \pi^{0}}^{B T} R_{A c c}(1 \gamma / 2 \gamma) \frac{D_{f}^{e p \pi^{0}}}{D_{f}^{e p \gamma}}\right)^{2}} \\
\delta R_{A c c}(1 \gamma / 2 \gamma)=R_{A c c}(1 \gamma / 2 \gamma) \sqrt{\left(\frac{\delta N_{r e c}^{e p \pi^{0}(1 \gamma)}}{N_{r e c}^{e p \pi^{0}(1 \gamma)}}\right)^{2}+\left(\frac{\delta N_{r e c}^{e p \pi^{0}(2 \gamma)}}{N_{r e c}^{e p \pi^{0}}(2 \gamma)}\right)^{2}}
\end{gathered}
$$

where $N_{r e c}^{e p \pi^{0}(1 \gamma)}$ and $N_{r e c}^{e p \pi^{0}(2 \gamma)}$ are the number of GSIM reconstructed events for single and two detected photon $\pi^{0}$ electroproduction. The partial derivatives of Equation 5.5 are defined as:

$$
\begin{aligned}
& \frac{\partial A_{U L}}{\partial N^{\uparrow \Uparrow}}=\frac{\left(N^{\downarrow \Downarrow}+N^{\uparrow \Downarrow}\right)\left(P_{t}^{\Downarrow}+P_{t}^{\Uparrow}\right)}{D_{f}\left(\left(N^{\downarrow \Uparrow}+N^{\uparrow \Uparrow}\right) P_{t}^{\Downarrow}+\left(N^{\downarrow \Downarrow}+N^{\uparrow \Downarrow}\right) P_{t}^{\Uparrow}\right)^{2}} \\
& \frac{\partial A_{U L}}{\partial N^{\downarrow \Downarrow}}=\frac{-\left(N^{\downarrow \Uparrow}+N^{\uparrow \Uparrow}\right)\left(P_{t}^{\Downarrow}+P_{t}^{\Uparrow}\right)}{D_{f}\left(\left(N^{\downarrow \Uparrow}+N^{\uparrow \Uparrow}\right) P_{t}^{\Downarrow}+\left(N^{\downarrow \Downarrow}+N^{\uparrow \Downarrow}\right) P_{t}^{\Uparrow}\right)^{2}} \\
& \frac{\partial A_{U L}}{\partial N^{\uparrow \Downarrow}}=\frac{-\left(N^{\downarrow \Uparrow}+N^{\uparrow \Uparrow}\right)\left(P_{t}^{\Downarrow}+P_{t}^{\Uparrow}\right)}{D_{f}\left(\left(N^{\downarrow \uparrow}+N^{\uparrow \Uparrow}\right) P_{t}^{\Downarrow}+\left(N^{\downarrow \Downarrow}+N^{\uparrow \Downarrow}\right) P_{t}^{\Uparrow}\right)^{2}} \\
& \frac{\partial A_{U L}}{\partial N^{\downarrow \Uparrow}}=\frac{\left(N^{\downarrow \Downarrow}+N^{\uparrow \Downarrow}\right)\left(P_{t}^{\Downarrow}+P_{t}^{\Uparrow}\right)}{D_{f}\left(\left(N^{\downarrow \Uparrow}+N^{\uparrow \Uparrow}\right) P_{t}^{\Downarrow}+\left(N^{\downarrow \Downarrow}+N^{\uparrow \Downarrow}\right) P_{t}^{\Uparrow}\right)^{2}} .
\end{aligned}
$$

\subsection{Target-Spin Asymmetry Results}

The final target-spin asymmetries from EG1-DVCS Run Periods A and B are shown superimposed for each of the kinematic bins discussed in Section 3.7 in Figures 5.1 - 5.5. 

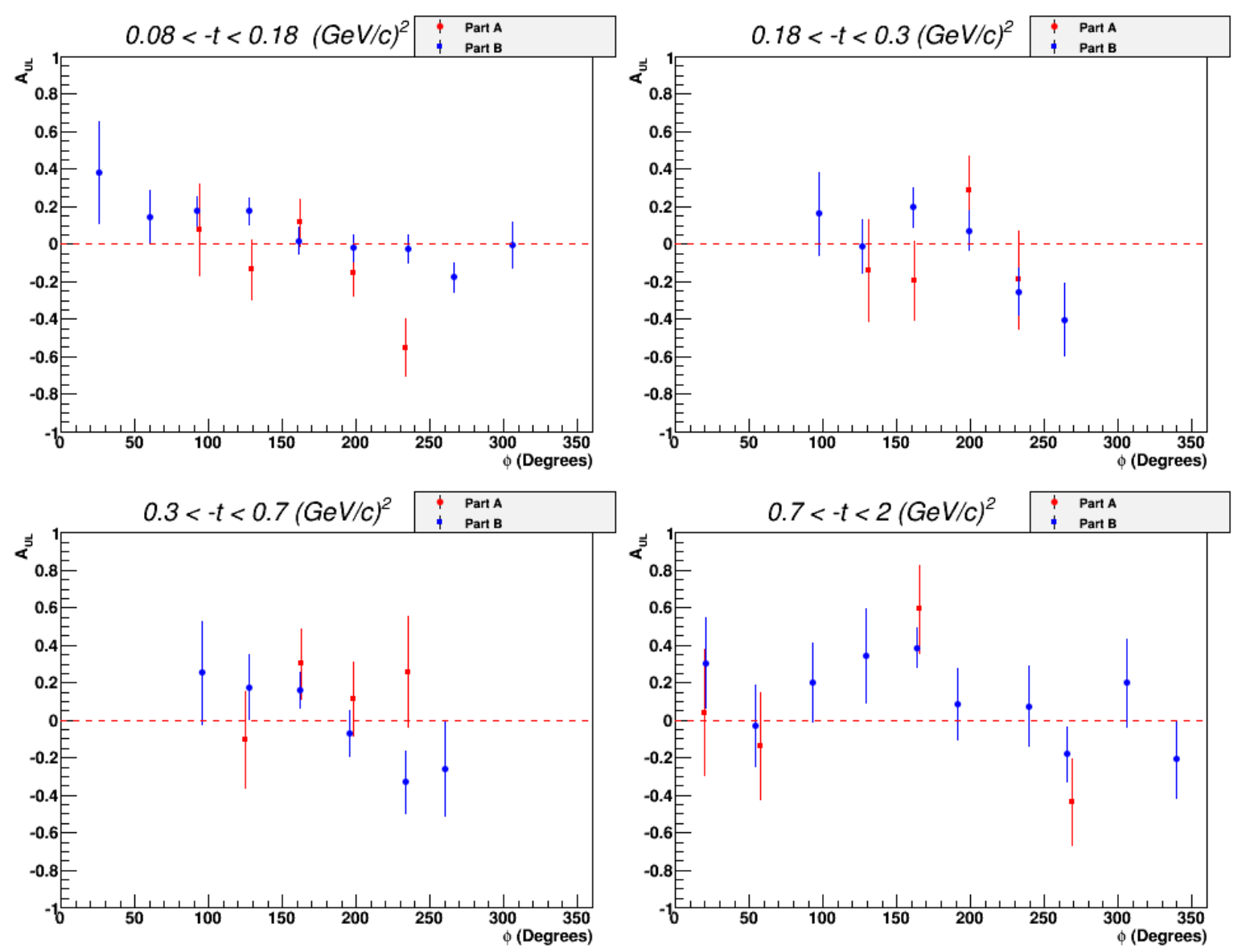

Fig. 5.1: Target-spin asymmetry, as a function of $\phi$, for each bin in $-t$, for the first bin in $Q^{2}-x_{B}$. The red and blue points represent, respectively, the asymmetries for EG1-DVCS Run Periods A and B. Error bars shown are statistical. 

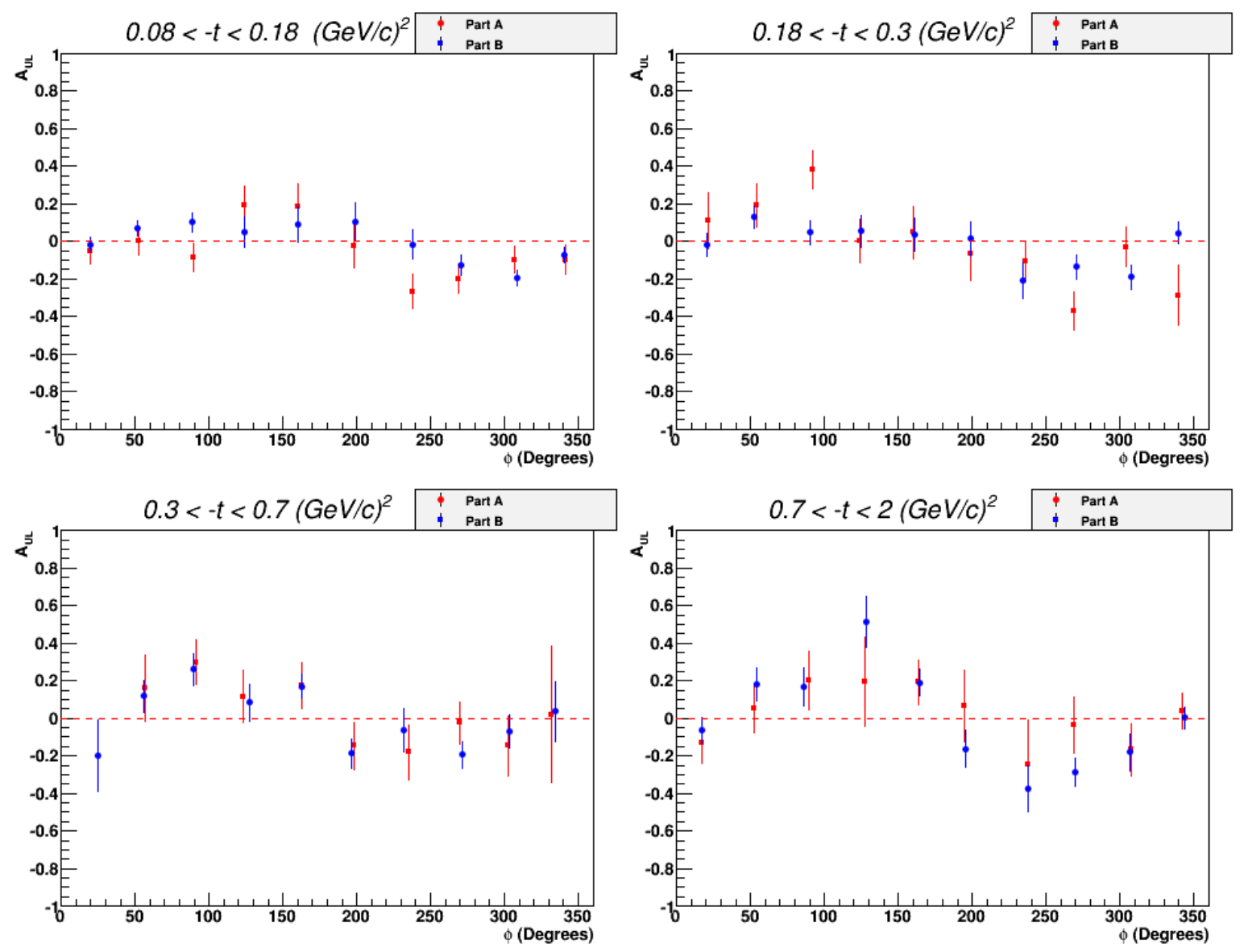

Fig. 5.2: Target-spin asymmetry, as a function of $\phi$, for each bin in $-t$, for the second bin in $Q^{2}-x_{B}$. The red and blue points represent, respectively, the asymmetries for EG1-DVCS Run Periods A and B. Error bars shown are statistical. 

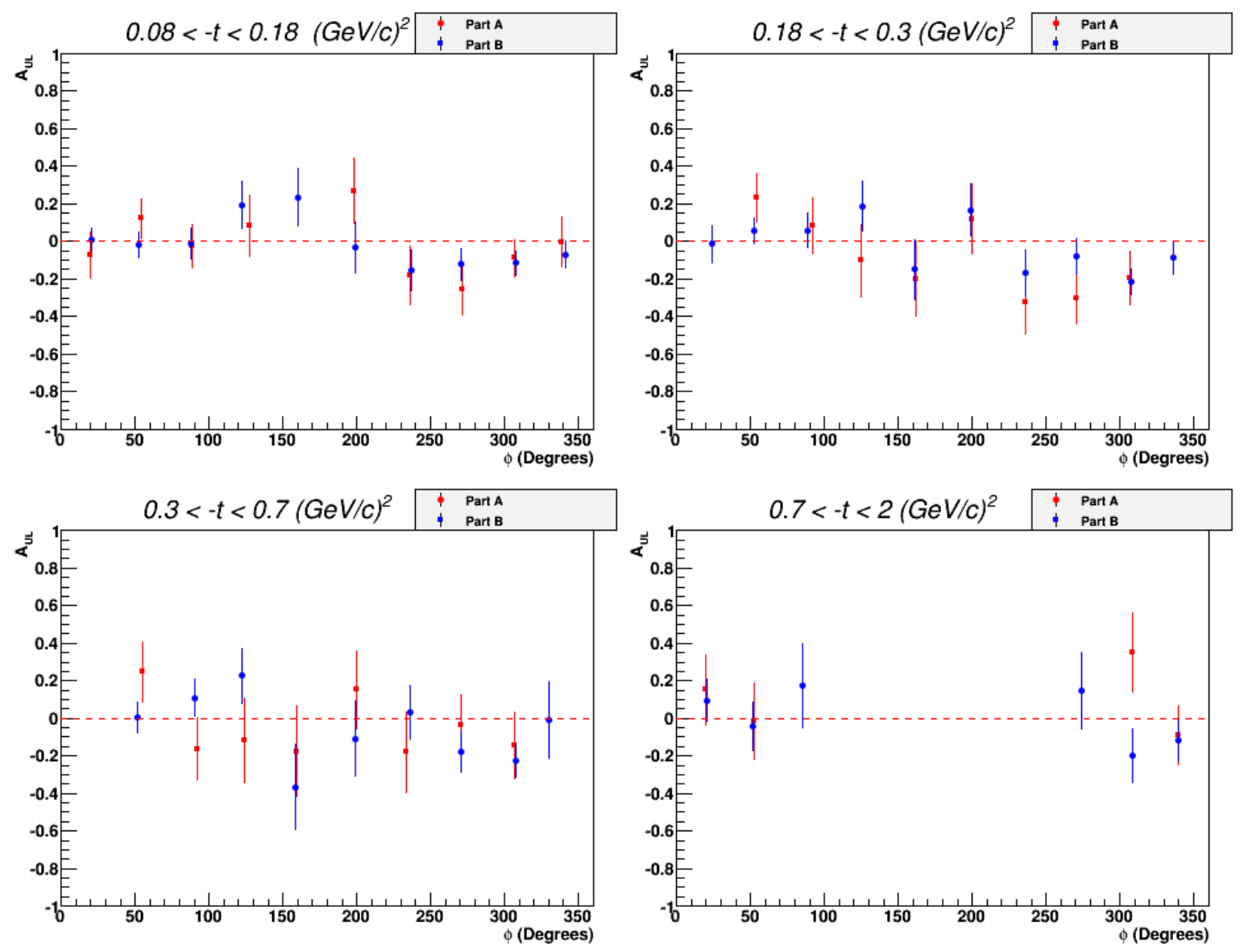

Fig. 5.3: Target-spin asymmetry, as a function of $\phi$, for each bin in $-t$, for the third bin in $Q^{2}-x_{B}$. The red and blue points represent, respectively, the asymmetries for EG1-DVCS Run Periods A and B. Error bars shown are statistical. 

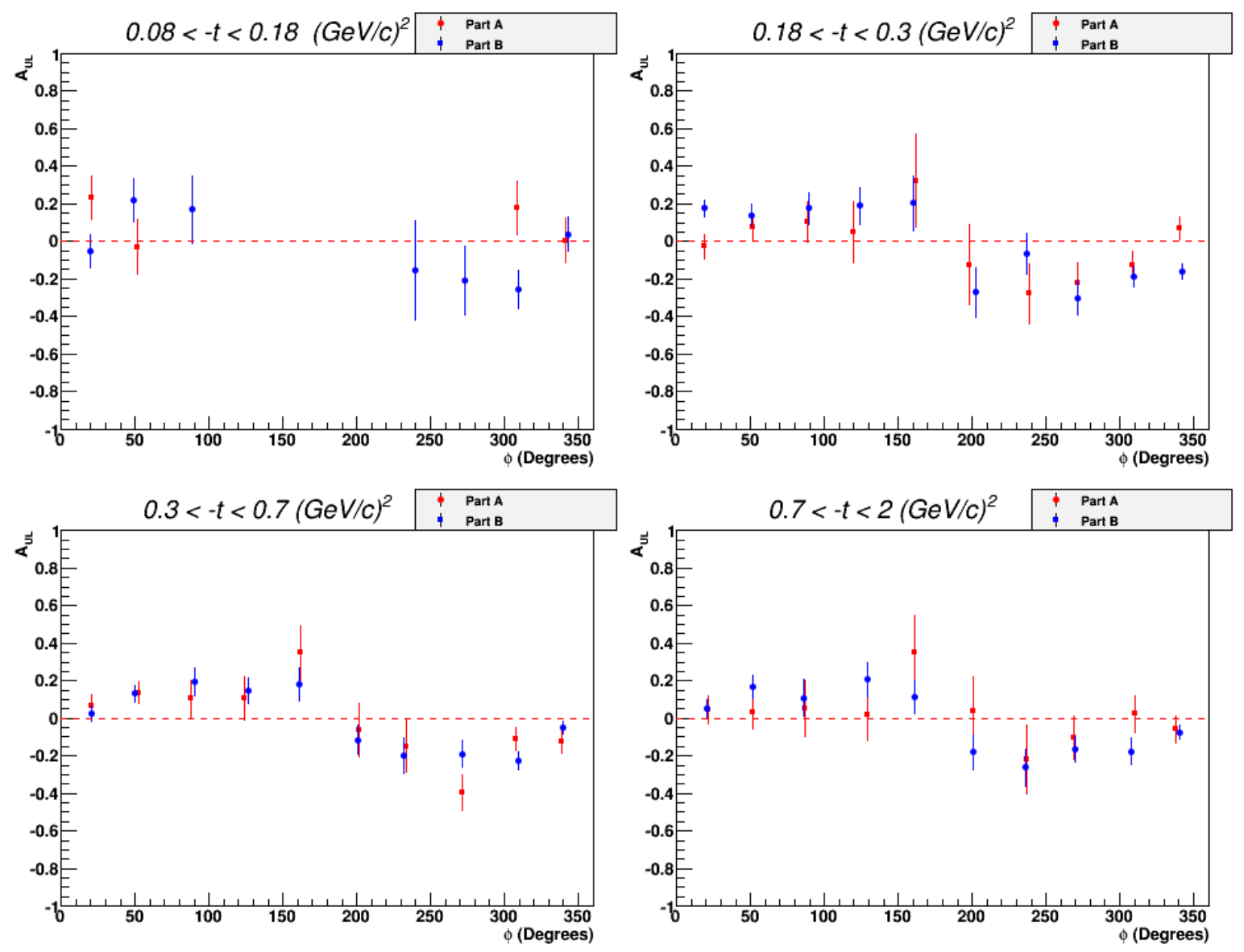

Fig. 5.4: Target-spin asymmetry, as a function of $\phi$, for each bin in $-t$, for the fourth bin in $Q^{2}-x_{B}$. The red and blue points represent, respectively, the asymmetries for EG1-DVCS Run Periods A and B. Error bars shown are statistical. 

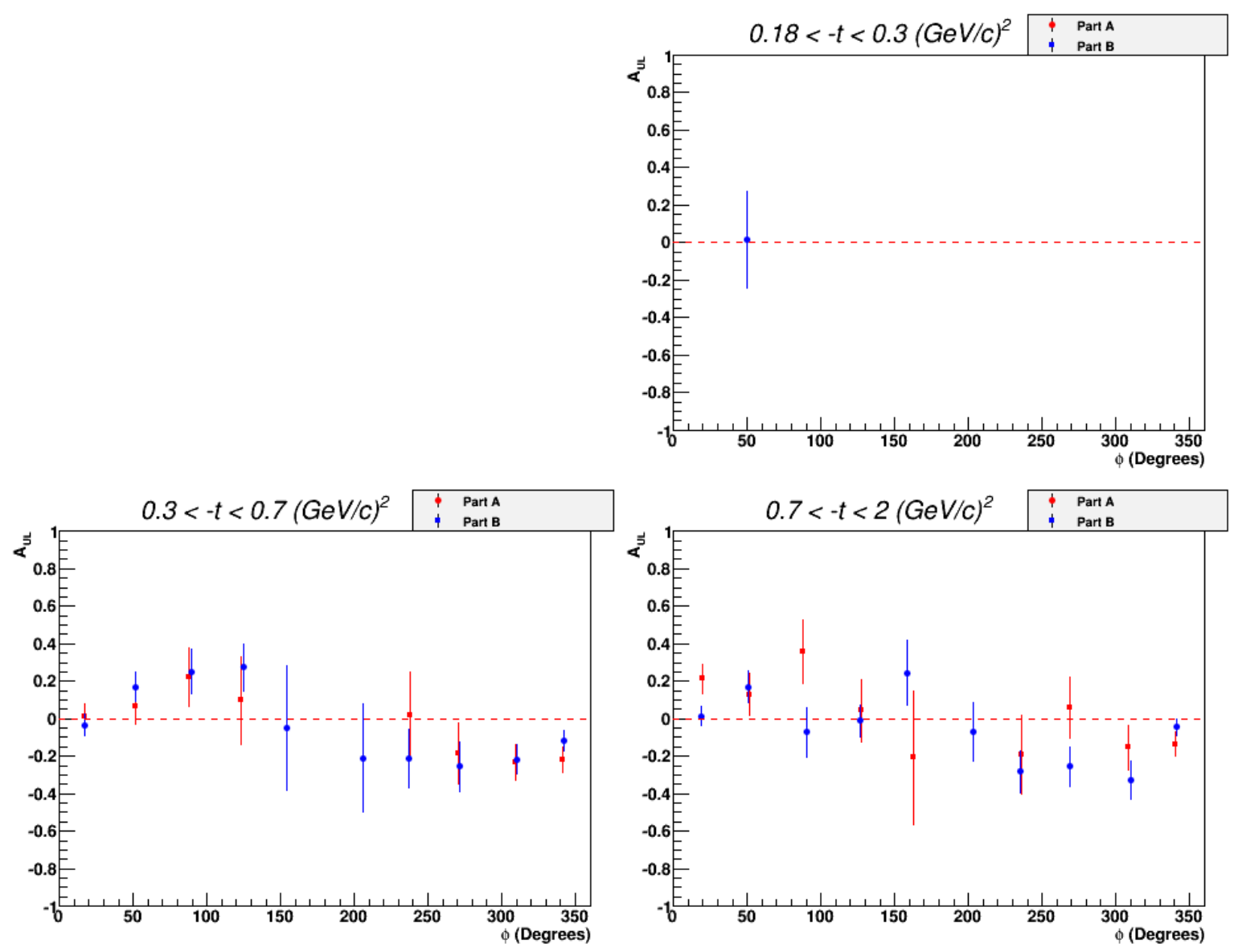

Fig. 5.5: Target-spin asymmetry, as a function of $\phi$, for each bin in $-t$, for the fifth bin in $Q^{2}-x_{B}$. The red and blue points represent, respectively, the asymmetries for EG1-DVCS Run Periods A and B. Error bars shown are statistical. 


\subsection{Combination of EG1-DVCS Run Periods A and B}

Two studies were performed in order to verify that the asymmetries from the two run periods of the EG1-DVCS Experiment were statistically compatible with each other and therefore could be combined. The first study was a t-test,

$$
t=\frac{A_{U L}^{A}-A_{U L}^{B}}{\operatorname{sqrt}\left(\delta A_{U L}^{A}\right)^{2}+\left(\delta A_{U L}^{B}\right)^{2}}
$$

If the fluctuations between EG1-DVCS Run Periods A and B are purely statistical, we expect that the distribution of $t$ for the different bins is a Gaussian with a mean of zero and a standard deviation (which is similar to $\chi^{2}$ ) of 1 . The mean $t$ should be zero within the error on the mean, which is simply $1 / \sqrt{N_{\text {bins }}}$. Figure 5.6 shows the t-test distribution with a mean value of $t$ that is compatible within the error with 0 , and a standard deviation close to 1 .

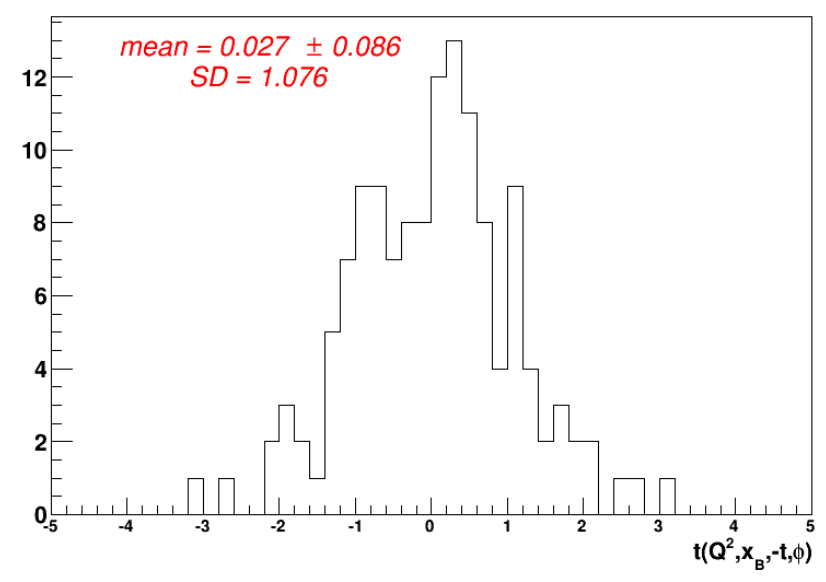

Fig. 5.6: t-test distribution, as defined in Equation 5.12.

The second study was to look at the acceptance in each kinematic bin, it was found that the data averaged mean as well as the Monte Carlo averaged mean in each bin for the two different run periods was well within the standard deviation, as shown for $Q^{2}$ vs $x_{B}$ in Figure 5.7 and for $-t$ vs $x_{B}$ in Figure 5.9. 

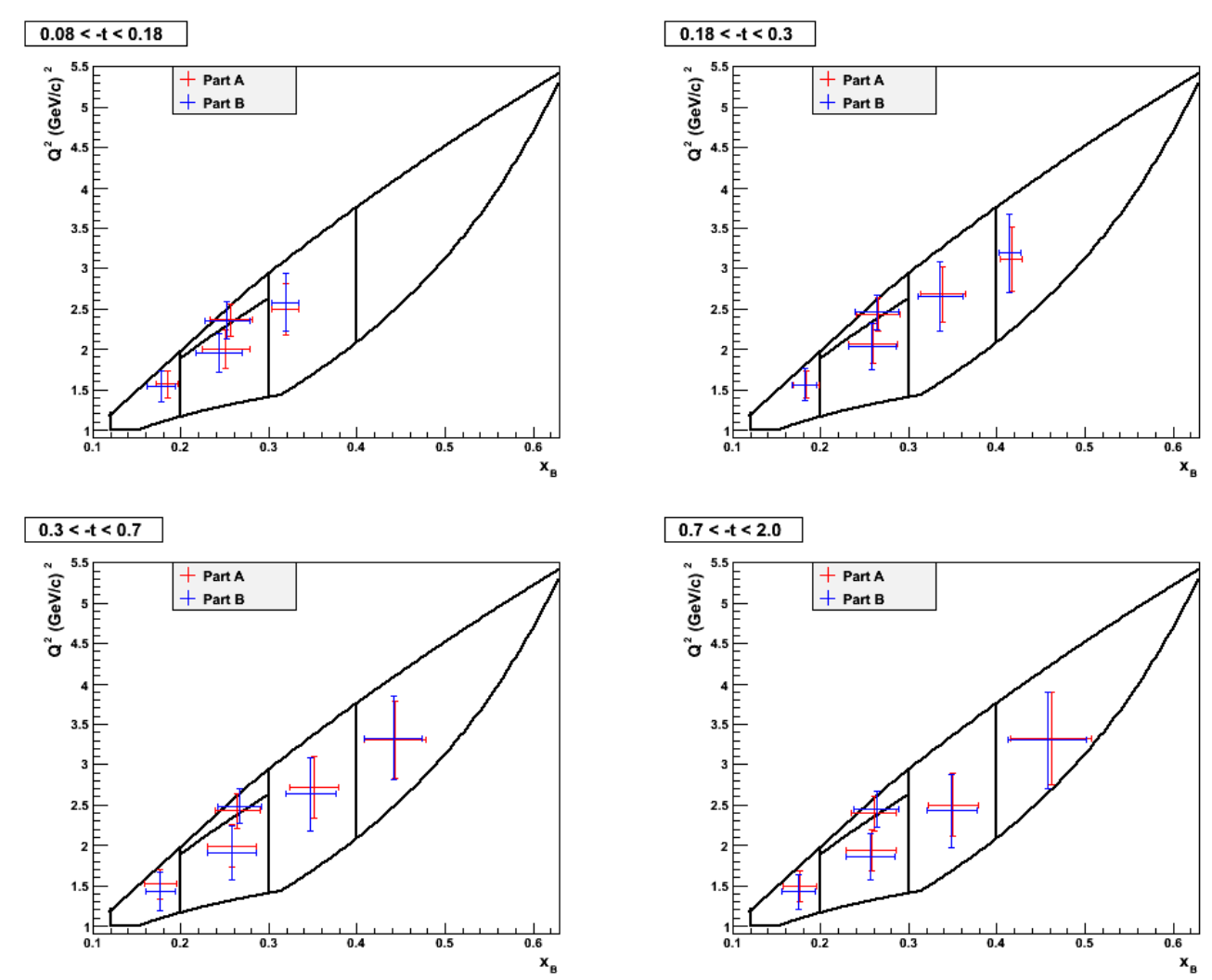

Fig. 5.7: Data average values of $Q^{2}$ (y axis) and $x_{B}$ (x axis) for each kinematic bin in the $Q^{2}-x_{B}$ space, and for every bin in $-t$. The red and blue points correspond respectively, to EG1-DVCS Run Periods A and B. The error bars are the standard deviations. 

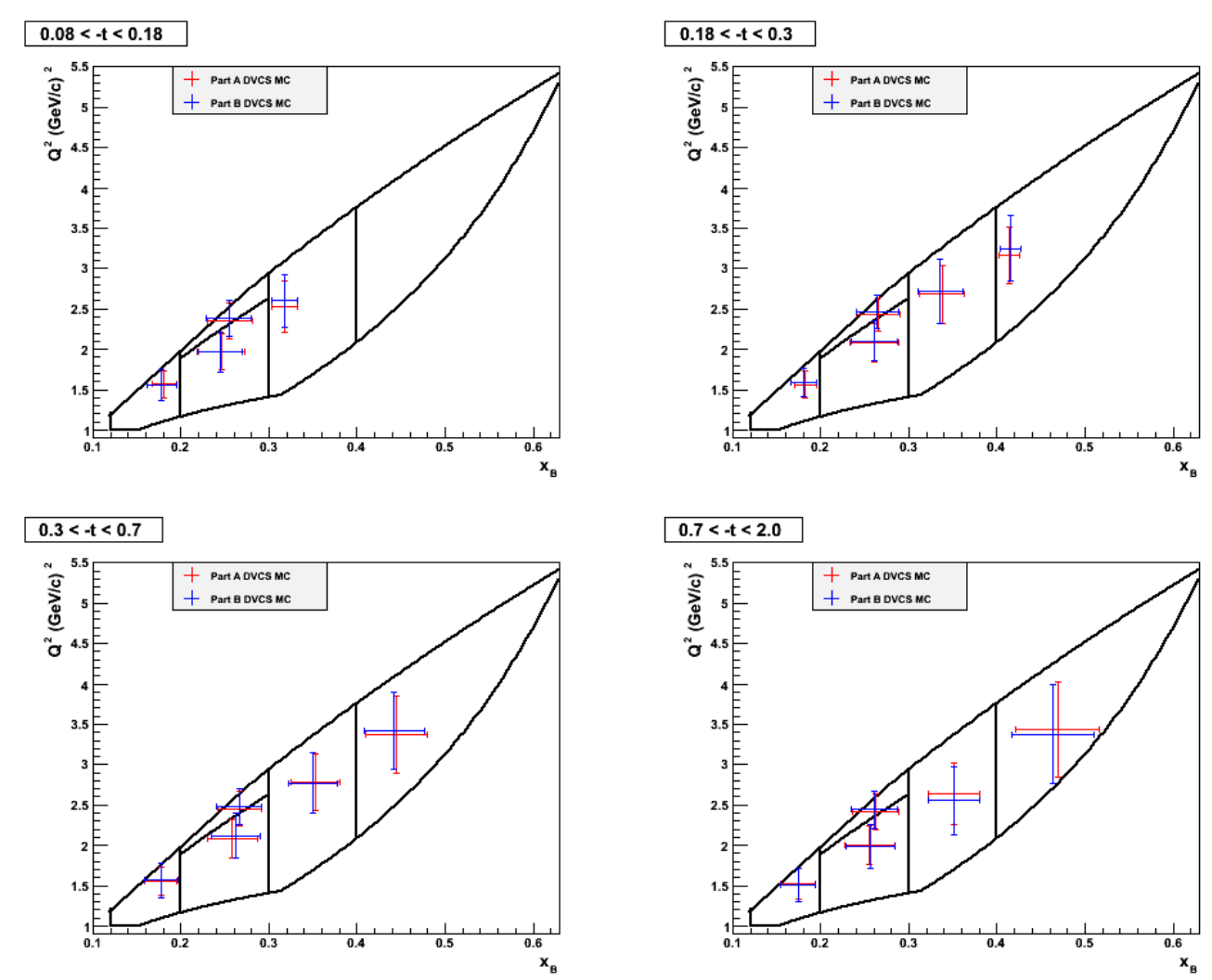

Fig. 5.8: DVCS/Bethe-Heitler Monte-Carlo simulation average values of $Q^{2}$ (y axis) and $x_{B}$ (x axis) for each kinematic bin in the $Q^{2}-x_{B}$ space, and for every bin in $-t$. The red and blue points correspond respectively, to EG1-DVCS Run Periods A and B. The error bars are the standard deviations. 


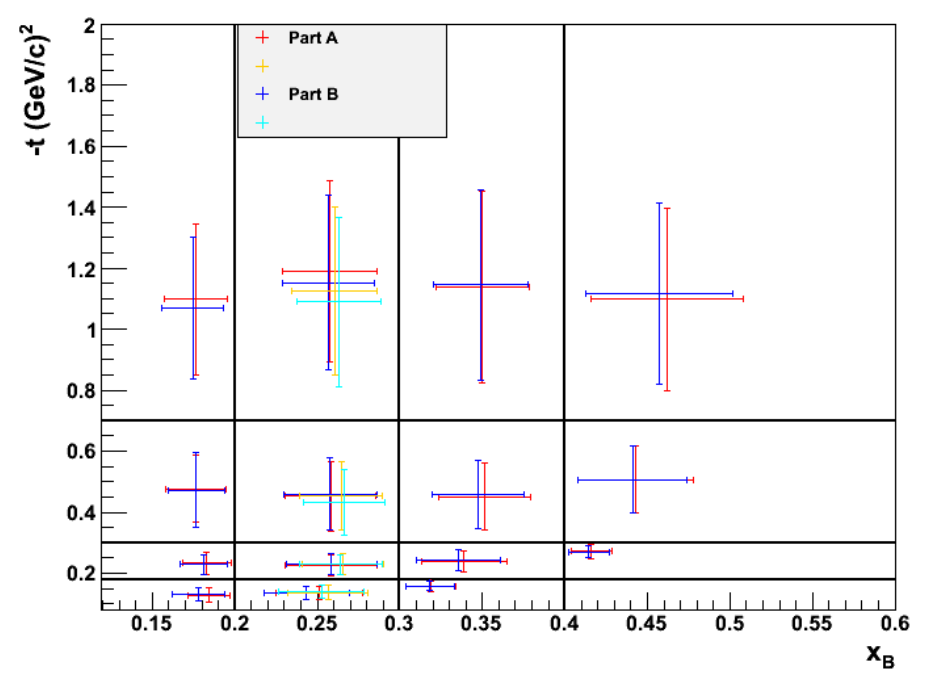

Fig. 5.9: Data average values of $-t$ (y axis) and $x_{B}$ (x axis) for each kinematic bin in the $-t-x_{B}$ space. The red (and yellow) and blue (and cyan) points correspond respectively, to EG1-DVCS Run Periods A and B. The two points in yellow and cyan correspond to the second bin in $Q^{2}$ of that same $x_{B}$ bin. The error bars are the standard deviations. 


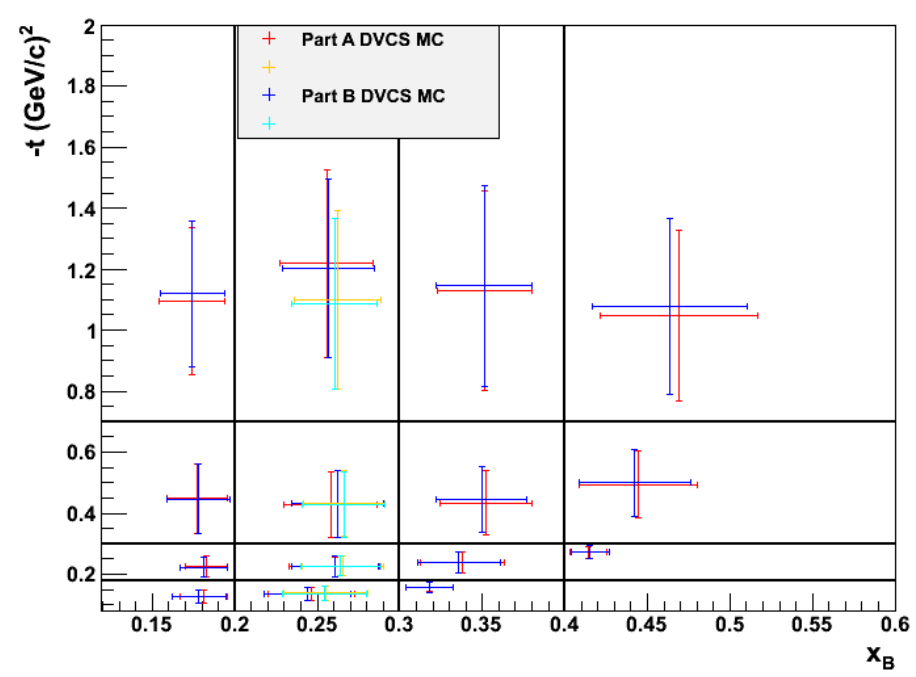

Fig. 5.10: DVCS/Bethe-Heitler Monte-Carlo simulation average values of $-t$ (y axis) and $x_{B}$ (x axis) for each kinematic bin in the $-t-x_{B}$ space. The red (and yellow) and blue (and cyan) points correspond respectively, to EG1-DVCS Run Periods A and $\mathrm{B}$. The two points in yellow and cyan correspond to the second bin in $Q^{2}$ of that same $x_{B}$ bin. The error bars are the standard deviations. 


\subsection{Merged Asymmetries}

The two run periods were combined using a weighted average, Equation 5.13. Figures 5.115.15 show the final merged results for the DVCS-BH target-spin asymmetry, as a function of $\phi$, for each bin in $-t$ and for each slice in the $Q^{2}-x_{B}$ space. The data are fit with the function $\alpha \sin \phi+\beta \sin 2 \phi$ (blue curve, blue parameters).

$$
\begin{gathered}
A_{U L}=\frac{\frac{A_{U L}^{A}}{\left(\delta A_{U L}^{A}\right)^{2}}+\frac{A_{U L}^{B}}{\left(\delta A_{U L}^{B}\right)^{2}}}{\frac{1}{\left(\delta A_{U L}^{A}\right)^{2}}+\frac{1}{\left(\delta A_{U L}^{B}\right)^{2}}} \\
\delta A_{U L}^{2}=\frac{1}{\frac{1}{\left(\delta A_{U L}^{A}\right)^{2}}+\frac{1}{\left(\delta A_{U L}^{B}\right)^{2}}}
\end{gathered}
$$
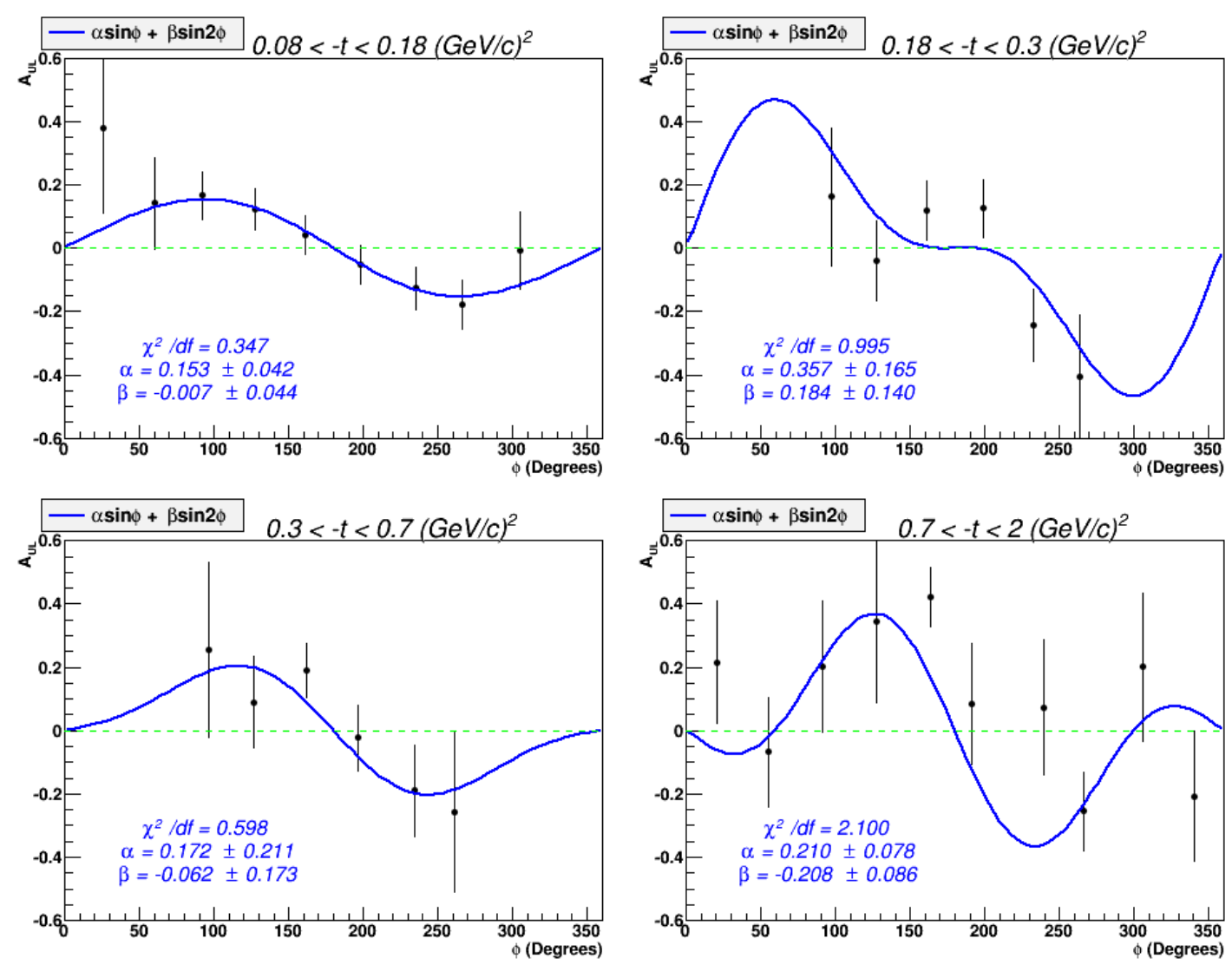

Fig. 5.11: Target-spin asymmetry for DVCS-BH events, as a function of $\phi$, for the various $-t$ bins, for the first bin in $Q^{2}-x_{B}$. 

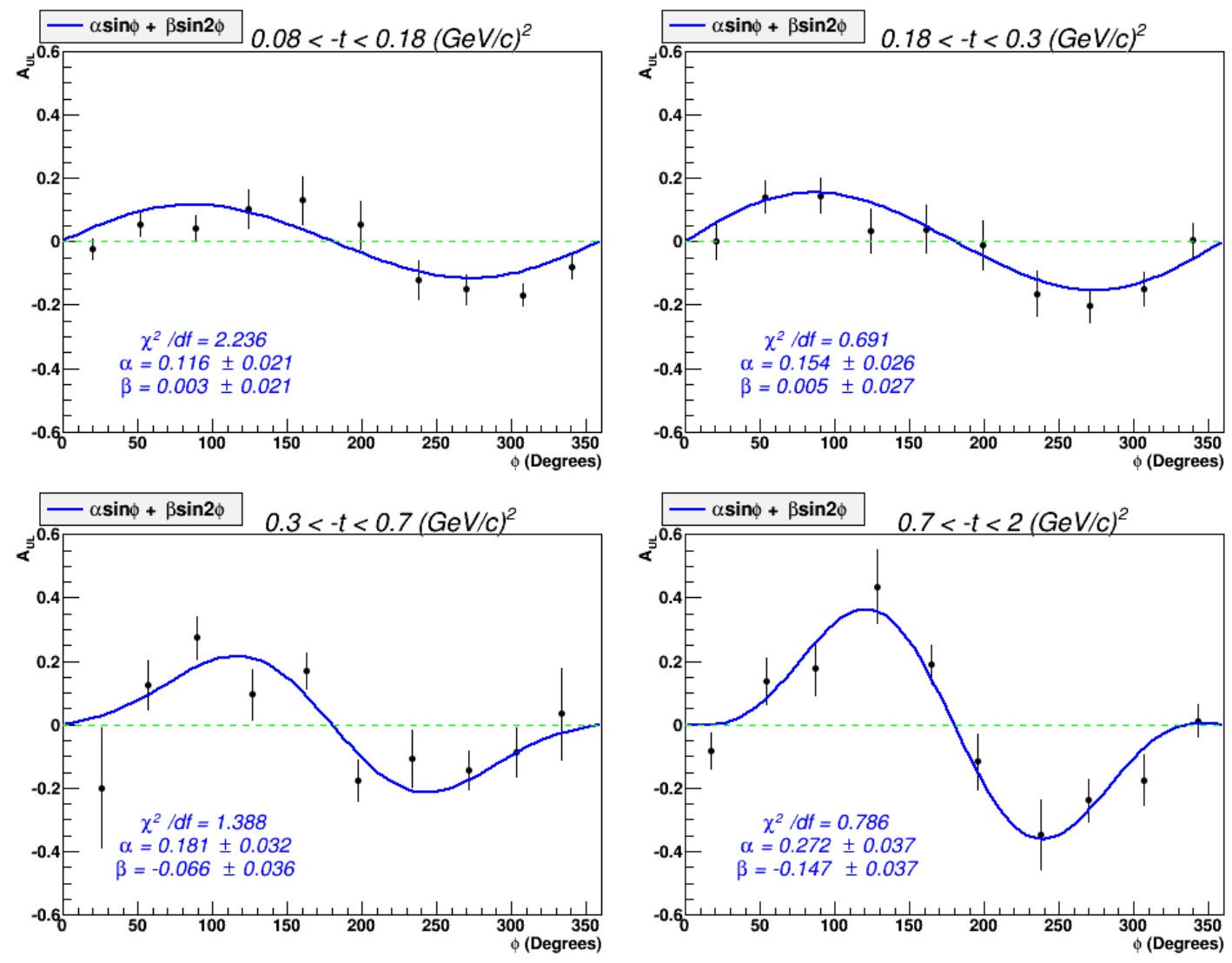

Fig. 5.12: Target-spin asymmetry for DVCS-BH events, as a function of $\phi$, for the various $-t$ bins, for the second bin in $Q^{2}-x_{B}$. 

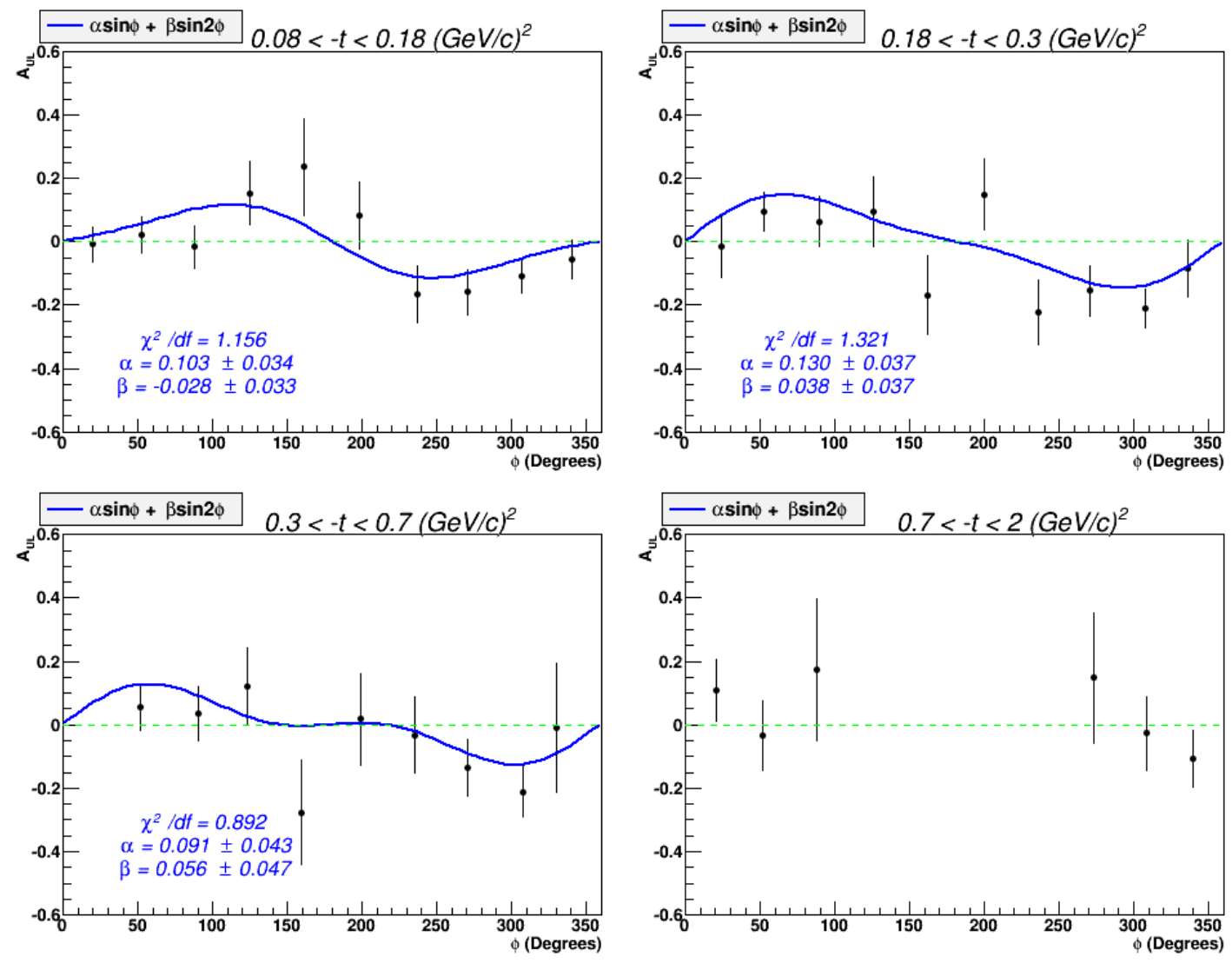

Fig. 5.13: Target-spin asymmetry for DVCS-BH events, as a function of $\phi$, for the various $-t$ bins, for the third bin in $Q^{2}-x_{B}$. 

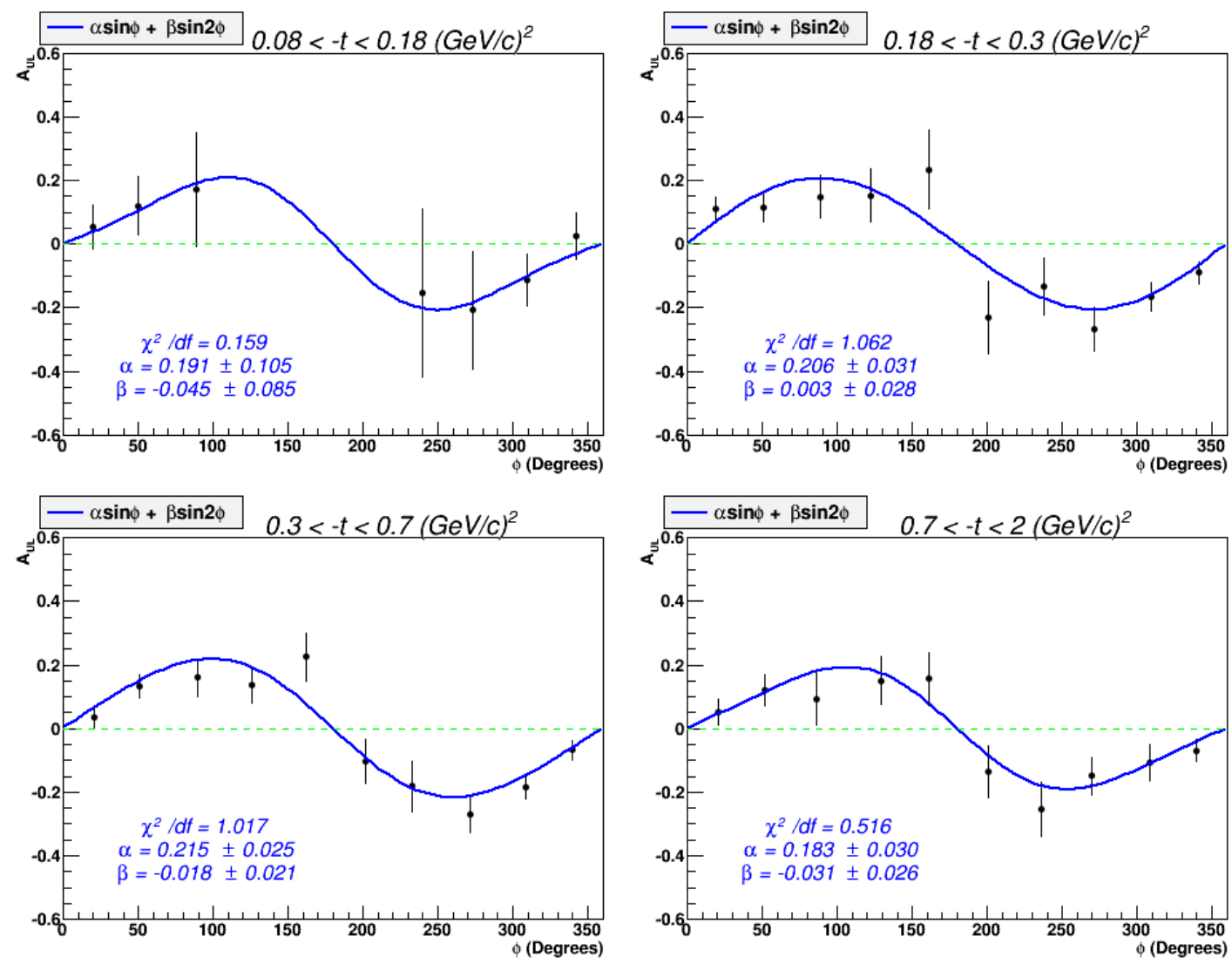

Fig. 5.14: Target-spin asymmetry for DVCS-BH events, as a function of $\phi$, for the various $-t$ bins, for the fourth bin in $Q^{2}-x_{B}$. 

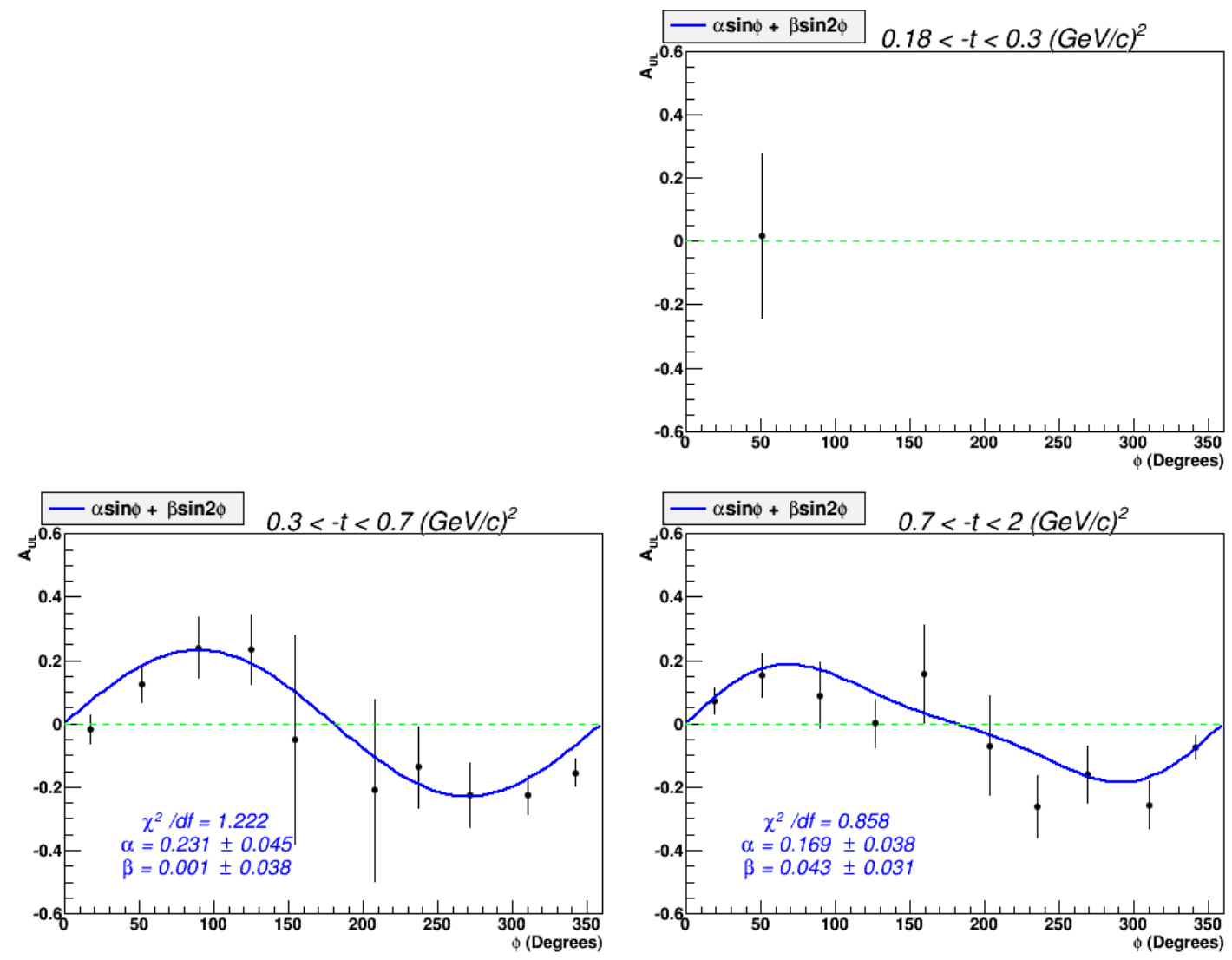

Fig. 5.15: Target-spin asymmetry for DVCS-BH events, as a function of $\phi$, for the various $-t$ bins, for the fifth bin in $Q^{2}-x_{B}$. 
Figure 5.16 shows the $t$-dependency, for each bin in $Q^{2}-x_{B}$, of the $\sin \phi$ term, $\alpha$, for three different fitting functions: $\alpha \sin \phi$ (red), $\alpha \sin \phi /(1+\beta \cos \phi)$ (blue), $\alpha \sin \phi+\beta \sin (2 \phi)$ (green). The three sets of values of $\alpha$ are roughly in agreement, point-by-point, within error bars - although the agreement is much worse in the first $Q^{2}-x_{B}$ bin (especially in the second $t$ bin), where the statistics are lower and the $\phi$ coverage is not complete. It is important to note that the $\beta$ parameter in the $\alpha \sin \phi+\beta \sin (2 \phi)$ fit, which is associated with higher-twist effects, may mock some effects of the $(1+\beta \cos \phi)$ denominator at leading twist. Because of limited statistics a fit of $(\alpha \sin \phi+\beta \sin (2 \phi)) /(1+\gamma \cos \phi)$, which would be needed to differentiate the two terms was not possible.
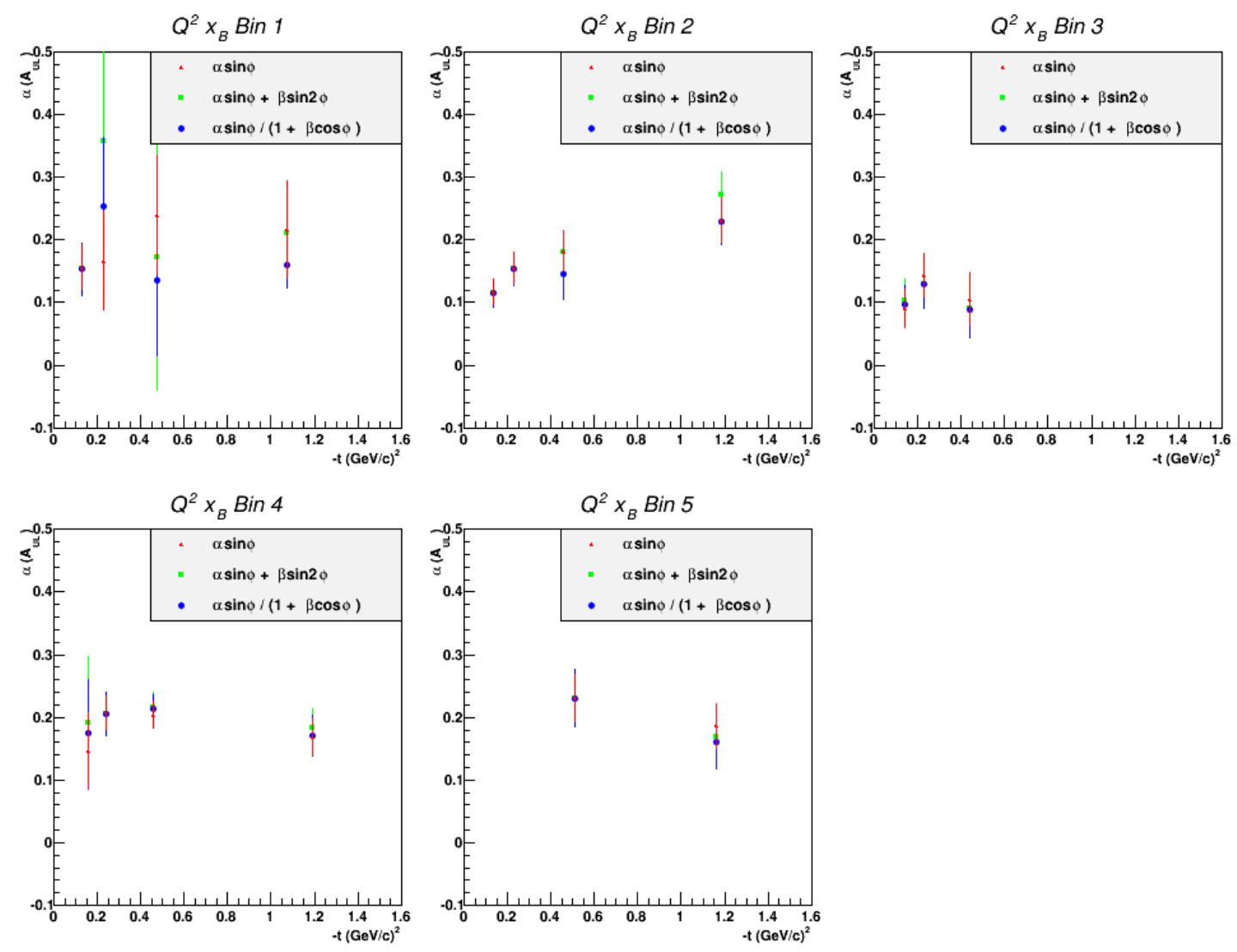

Fig. 5.16: $t$ dependence, for each $Q^{2}-x_{B}$ bin, of $\sin \phi$ term of the DVCS/BH targetspin asymmetry, for each $Q^{2}-x_{B}$ bin. The three colors indicate three different functions used to fit the $\phi$-dependent TSA. 
Figure 5.17 shows the $t$-dependency, for each bin in $Q^{2}-x_{B}$, of the $\sin \phi$ fitting coefficient compared to the predictions of the Vanderhaeghen, Guichon, Guidal (VGG) model [52] [53]. While the model reproduces the average magnitude of the asymmetry, it fails to correctly provide its $t$ dependence: the data in fact don't exhibit such a strong drop as $t$ increases as the one foreseen by the model.
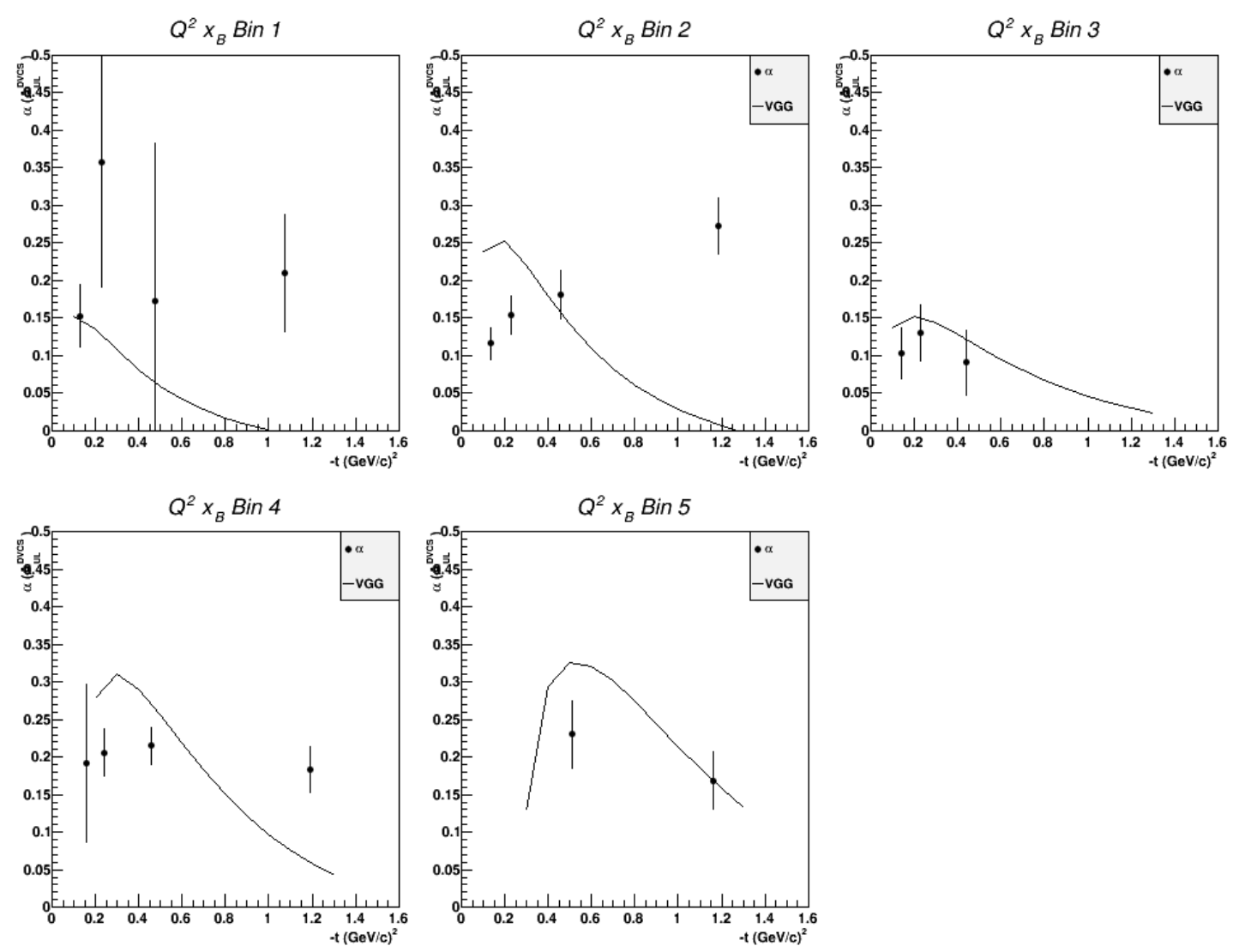

Fig. 5.17: $t$ dependence, for each $Q^{2}-x_{B}$ bin, of $\sin \phi$ term of the DVCS/BH target-spin asymmetry. The curves are the predictions of the VGG model for the TSA at $\phi=90^{\circ}$. 


\subsection{Systematic Errors}

Several systematic checks were performed to evaluate the stability of the measured targetspin asymmetry against the variation of the individual parts. The error was estimated by measuring the RMS of deviations on the values of $A_{U L}$ in each kinematic bin, $\Delta_{R M S}$, defined by:

$$
\Delta_{R M S}=\frac{\sqrt{\sum_{X} \Delta_{X}^{2}}}{\sqrt{N}}
$$

where $\Delta_{X}$ is the difference between the nominal $A_{U L}$ value and the $A_{U L}$ value calculated with deviation $X$, and $N$ is the number of deviations considered. The deviations for each source of error are discussed individually in Sections 5.6.1 through 5.6.7. Table 5.1 summarizes the sources of systematic errors with the corresponding average $\Delta_{R M S}$ values.

\begin{tabular}{|l|c|}
\hline Source of Uncertainty & Avg. $\Delta_{R M S}$ \\
\hline \hline $\mathrm{PbPt}$ & 0.0026 \\
\hline $\mathrm{Pb}$ & 0.0032 \\
\hline$D_{f}$ & 0.0009 \\
\hline$e p \pi^{0}$ background subtraction & 0.0057 \\
\hline DVCS Exclusivity cuts & 0.0097 \\
\hline Particle Identification & \\
\hline Electron ID (NPHE) & 0.0056 \\
Proton ID $(\beta)$ & 0.0031 \\
\hline Radiative corrections & 0.0002 \\
\hline
\end{tabular}

Table 5.1: Summary of the sources of systematic error with associated average error on the Target Spin Asymmetry.

\subsubsection{Systematics on $P_{b} P_{t}$}

The uncertainty on the value of $P_{b} P_{t}$, determined through $e p$ elastic electron-spin asymmetry as discussed in Section 4.2, was determined for Run Period A to be $\Delta\left(P_{b} P_{t}^{+}\right)=0.016$ on the positive target polarization, $P_{b} P_{t}^{+}$, and $\Delta\left(P_{b} P_{t}^{-}\right)=0.015$ on the negative target polarization, $P_{b} P_{t}^{-}$; for Run Period B, $\Delta\left(P_{b} P_{t}^{+}\right)=0.011$ on $P_{b} P_{t}^{+}$, and $\Delta\left(P_{b} P_{t}^{-}\right)=0.010$ on $P_{b} P_{t}^{-}$. For 
each run period the two maximum deviations were considered, one with both values of $P_{b} P_{t}$ at their deviation maximum values, i.e. $P_{b} P_{t}^{+}+\Delta\left(P_{b} P_{t}^{+}\right)$and $P_{b} P_{t}^{-}-\Delta\left(P_{b} P_{t}^{-}\right)$, for both run periods, and one with both values of $P_{b} P_{t}$ at their deviation minimum values, i.e. $P_{b} P_{t}^{+}-\Delta\left(P_{b} P_{t}^{+}\right)$and $P_{b} P_{t}^{-}+\Delta\left(P_{b} P_{t}^{-}\right)$, for both run periods. The resulting $\Delta_{R M S}$ was determined for each kinematic bin, with an average value of 0.0026 .

\subsubsection{Systematics on $P_{b}$}

The systematic error on $P_{b}$ by Møller measurements (Section 4.2.4) was determined to be $\Delta\left(P_{b}\right)=0.02$, obtained by studies with the half-wave plate in and out of the electron beam. The deviations used to estimate the systematic effects on $A_{U L}$ were $P_{b}+\Delta\left(P_{b}\right)$ and $P_{b}-\Delta\left(P_{b}\right)$. The resulting $\Delta_{R M S}$ was determined for each 4-dimensional kinematic bin, with an average value of 0.0032 .

\subsubsection{Systematics on Dilution Factor}

The dilution factor (Section 4.6) was studied as a function of the kinematics, $Q^{2}, x_{B},-t$, and $\phi$. While the distributions were fairly consistent with a constant, the variations lead to an estimated uncertainty of $\Delta\left(D_{f}\right)=0.009$ and $\Delta\left(D_{f}\right)=0.006$ for EG1-DVCS Run Periods $\mathrm{A}$ and $\mathrm{B}$ respectively. To study the effect of the uncertainty on $A_{U L}$, the two maximum deviations were considered, one with both run periods applying $D_{f}+\Delta\left(D_{f}\right)$ and one with both run periods applying $D_{f}-\Delta\left(D_{f}\right)$. The resulting $\Delta_{R M S}$ was determined for each 4dimensional kinematic bin, with an average value of 0.0009 .

\subsubsection{Systematics on $e p \pi^{0}$ Background Subtraction}

The model dependency on the ratio $R=\frac{R_{\pi^{0}(\gamma)}}{R_{\pi^{0}(\gamma \gamma)}}$ (Section 4.7) was estimated to give a global

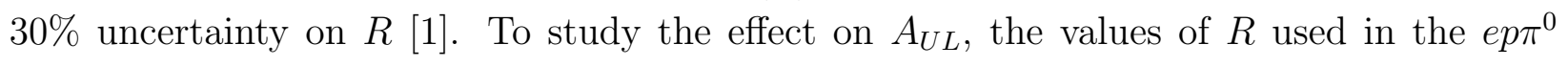
background subtraction were deviated by using $R \cdot 1.3$ and $R \cdot 0.7$. The resulting $\Delta_{R M S}$ was determined for each 4-dimensional kinematic bin, with an average value of 0.0057 . 


\subsubsection{Systematics on DVCS Exclusivity Selection}

Because the distributions used to select DVCS events (Section 3.5) are correlated, a study was done varying the selection criteria on all DVCS selection distributions from a $3 \sigma$ selection to a $2.5 \sigma$ and a $3.5 \sigma$ selection. Unlike the previously discussed systematic studies, a change in DVCS exclusivity selection changes the statistical sample, therefore the deviations in $A_{U L}$ are a combination of statistical and systematic effects. To minimize the statistical effects, the data were integrated over $Q^{2}$ and $x_{B}$, with $\Delta_{R M S}$ determined in the bins of $\phi$ and $-t$. The same method was applied to the $P_{b} P_{t}$ and $P_{b}$ systematic studies, which do not have a statistical difference with applied deviations, and it was found that the resulting $\Delta_{R M S}$ for those two studies was in good agreement with the values found using the 4-dimensional binning. Figure 5.18 shows an overlap of $A_{U L}$ determined with $3 \sigma$ DVCS selection (black), $2.5 \sigma$ DVCS selection (red), and 3.5 $\sigma$ DVCS selection (blue). The average $\Delta_{R M S}$ was determined to be 0.0096 . When done in the full 4-dimensional binning, the average $\Delta_{R M S}$ for the different selection schemes was 0.0333. Referencing the studies done on $P_{b} P_{t}$ and $P_{b}$, the difference in $\Delta_{R M S}$ between the 2-dimensional and 4-dimensional binning is attributed to statistical effects, and $\Delta_{R M S}=0.0097$ was determined to be the more accurate estimation of the systematics due to DVCS exclusivity selection. 

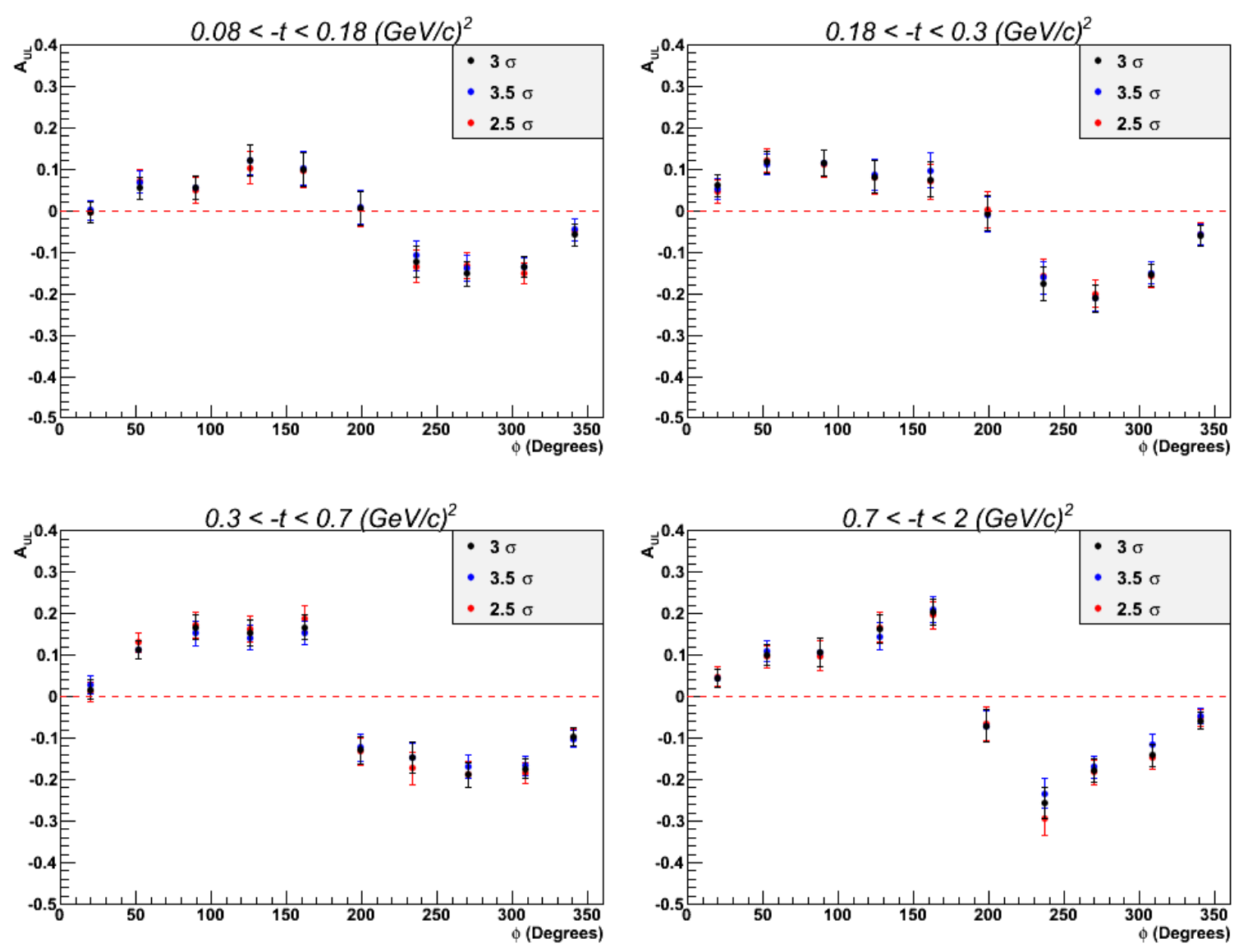

Fig. 5.18: $A_{U L}$, as a function of $\phi$, for 4 bins in $-t$. The black, blue and red points represent, respectively, the asymmetries for $3 \sigma, 3.5 \sigma$ and $2.5 \sigma$ DVCS exclusivity selection. Error bars shown are statistical. 


\subsubsection{Systematics on Particle Identification}

The particle identification cuts were chosen in this analysis to be very loose, relying on exclusive event selection cuts (DVCS, $e p \pi^{0}$, and $e p$ elastic) to select good events. There are two particle selection criteria used in this analysis that differ from previous DVCS analysis and lead to non-negligible systematic effects. The effect of these systematics are discussed individually in Sections 5.6.6.1 and 5.6.6.2.

\subsubsection{Systematics on Electron Selection: NPHE}

As was discussed in Section 3.2.1.10, no cut was used on number of photoelectrons (NPHE) in the Cerenkov counters for electrons in this analysis. The large peak around $10 \mathrm{x}$ NPHE $\sim 10$, attributed to a combination of $\pi^{-}$particles and good electrons which pass in angular regions where the produced photoelectrons miss the CC photomultiplier tubes. This peak was significantly reduced after all other electron identifications cuts (Figure 3.10). The DVCS exclusivity cuts were used to eliminate any residual $\pi^{-}$particles that passed particle identification so no cut on NPHE was used. However, in previous DVCS analysis (see References [54] and [55]), a minimum cut on NPHE was applied. Therefore it is important to study the systematics associated with the NPHE cut. A study was done using two different cuts on NPHE, one set at $10 \times$ NPHE $>15$ and one at $10 \times$ NPHE $>20$. Because a cut on NPHE changes the statistical sample, the deviations in $A_{U L}$ are a combination of statistical and systematic effects. To minimize the statistical effects, the data were integrated over $Q^{2}$ and $x_{B}$, with $\Delta_{R M S}$ determined in the bins of $\phi$ and $-t$, as was discussed in Section 5.6.5. The resulting average $\Delta_{R M S}$ was determined to be 0.0056 .

\subsubsection{Systematics on Proton Selection: $\beta$}

The proton selection used in this analysis involved a momentum dependent cut on $\beta$ (Section 3.2.2.3), with beta defined using SC timing and position information, as defined in Equation 5.15. There is an alternative selection, $\Delta \beta$, which uses the same momentum, $\mathrm{SC}$ timing and position information, but places a cut on the difference between $\beta$ calculated using SC timing (Equation 5.15) and the expected $\beta$ of a proton with momentum, $p$, as defined in 
Equation 5.16. Following previous DVCS analysis (see References [54] and [55]), a cut on $\Delta \beta$ of \pm 0.05 was chosen for this study. The difference in between the $\beta$ and $\Delta \beta$ selection is show in Figure 5.19, with the red lines indicating the cut used in this analysis, Section 3.2.2.3, and the alternative cut discussed above indicated in yellow. The resulting average $\Delta_{R M S}$ was determined to be 0.0031 .

$$
\begin{aligned}
& \beta=\frac{s c_{r}}{\left(s c_{t}-t_{\text {event }}\right) * c} \\
& \beta=\frac{p}{\sqrt{p * p+M * M}}
\end{aligned}
$$

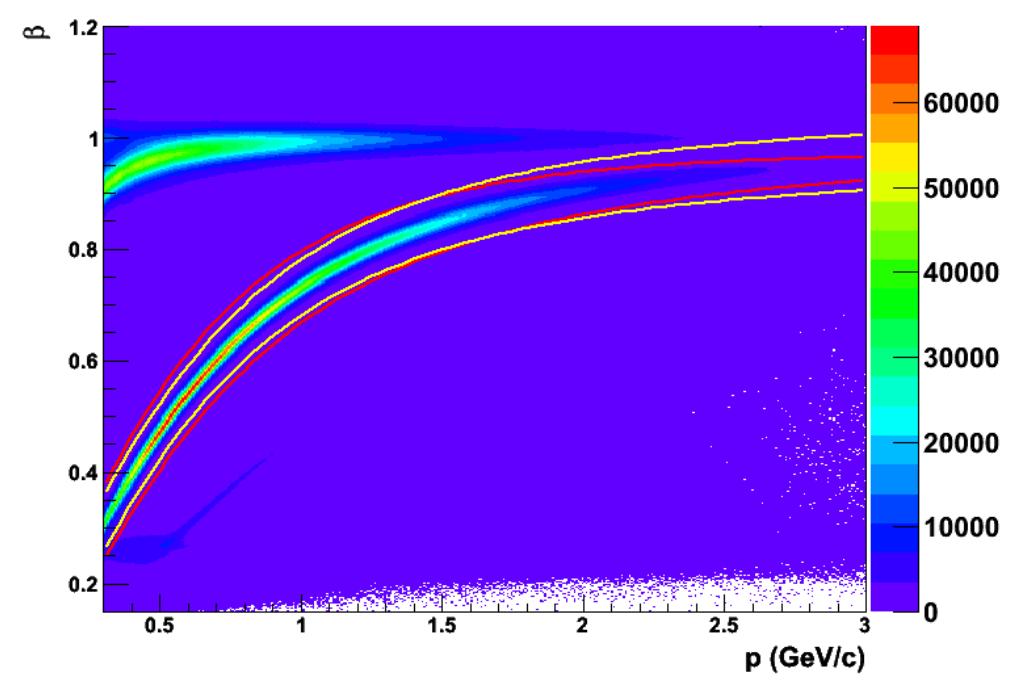

Fig. 5.19: $\beta$ distribution of proton candidates as a function of momentum. Red lines indicate the cut used in this analysis, Section 3.2.2.3, and the alternative cut considered for systematics indicated in yellow. 


\subsubsection{Systematics on Radiative Corrections}

Afanasev et al. [56] have computed the radiative corrections for the DVCS/BH process, for CLAS kinematics. It was found that, given the strict kinematic cuts adopted to select the final state, the undetected radiated photon can only have small energies. In this case, therefore, the main contribution to the radiative correction comes from spin-independent soft-photon emission that does not affect polarization observables - while instead it can affect unpolarized cross sections, even up to $20 \%$ level. The approximation of negligible contribution from the radiative corrections to $A_{U L}$, compared to the size of the asymmetries, is said to be valid at CLAS kinematics at the $0.1 \%$ level [57]. This translates to an average uncertainty of 0.0002 in $A_{U L}$.

\subsubsection{Total Systematic Error on $A_{U L}$}

Figures 5.20 through 5.24 show the target-spin asymmetry, $A_{U L}$, with associated statistical and systematic errors, as a function of $\phi$ for each bin in $-t$ and for each slice in the $Q^{2}-x_{B}$ space. The systematic errors, shown in grey, are the individual errors discussed in Sections 5.6.1 through 5.6.7 added in quadrature for each kinematic bin. 

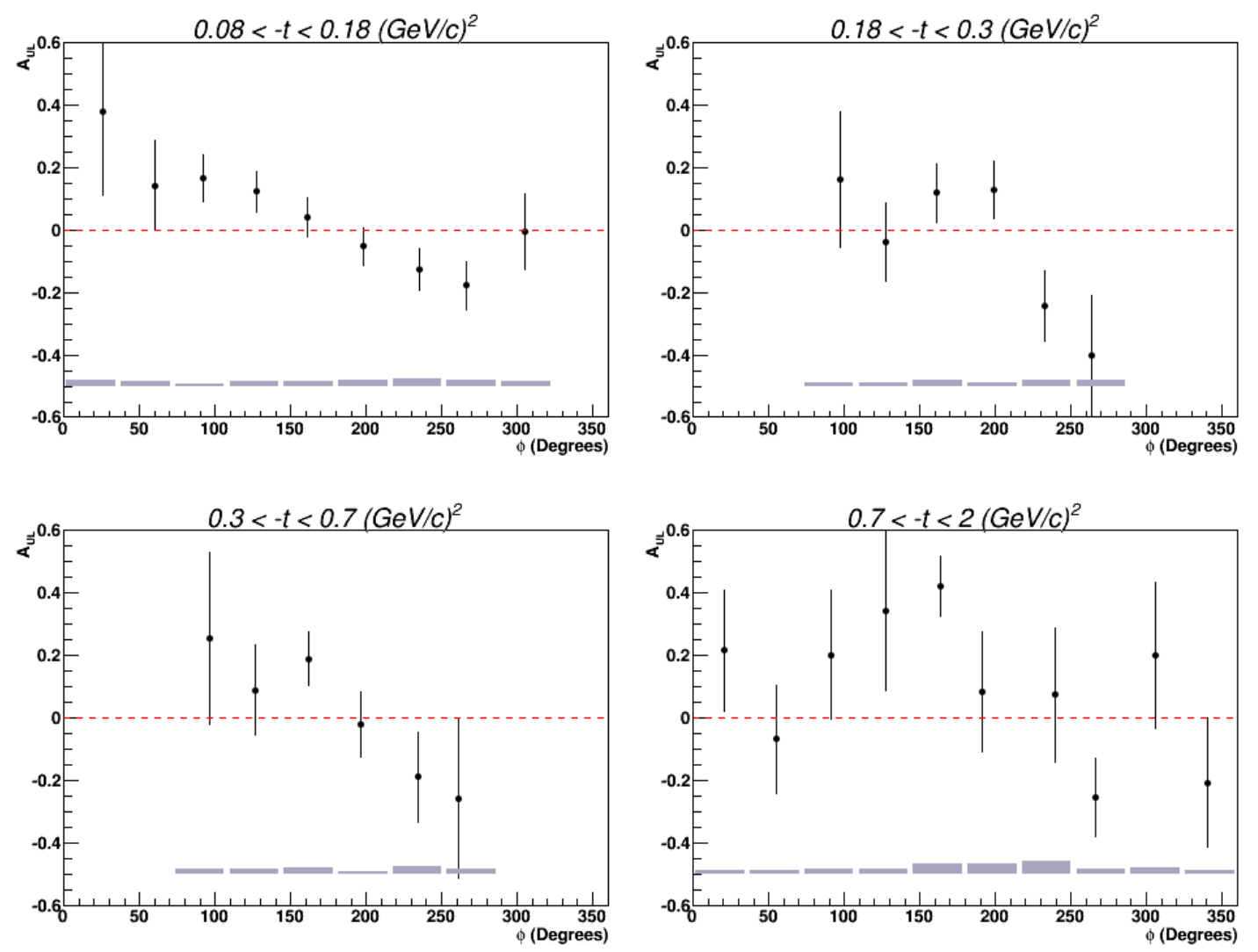

Fig. 5.20: Target-spin asymmetry for DVCS-BH events, as a function of $\phi$, for the various $-t$ bins, for the first bin in $Q^{2}-x_{B}$. The vertical lines indicate the statistical error, and the gray areas indicate the systematic error. 

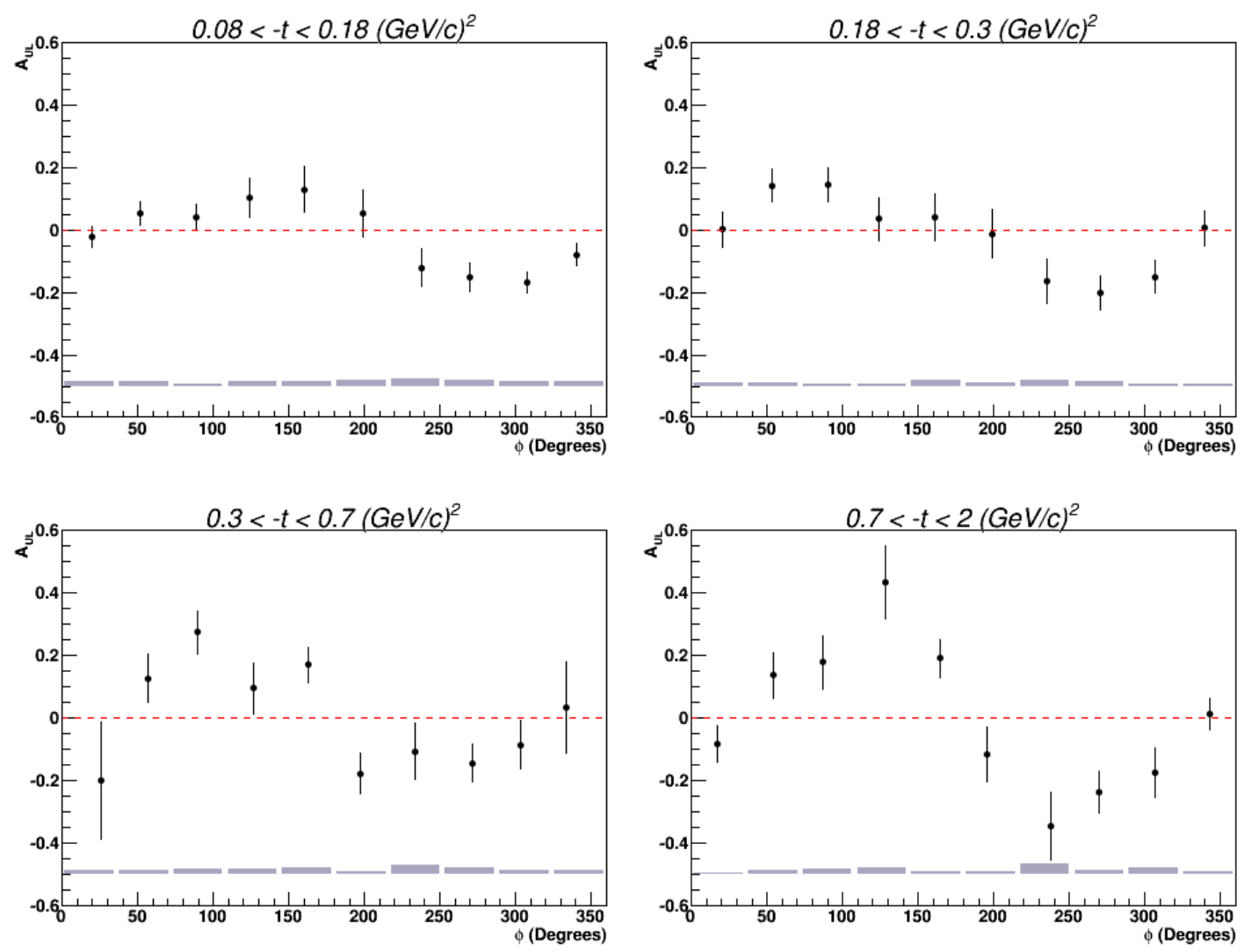

Fig. 5.21: Target-spin asymmetry for DVCS-BH events, as a function of $\phi$, for the various $-t$ bins, for the second bin in $Q^{2}-x_{B}$. The vertical lines indicate the statistical error, and the gray areas indicate the systematic error. 

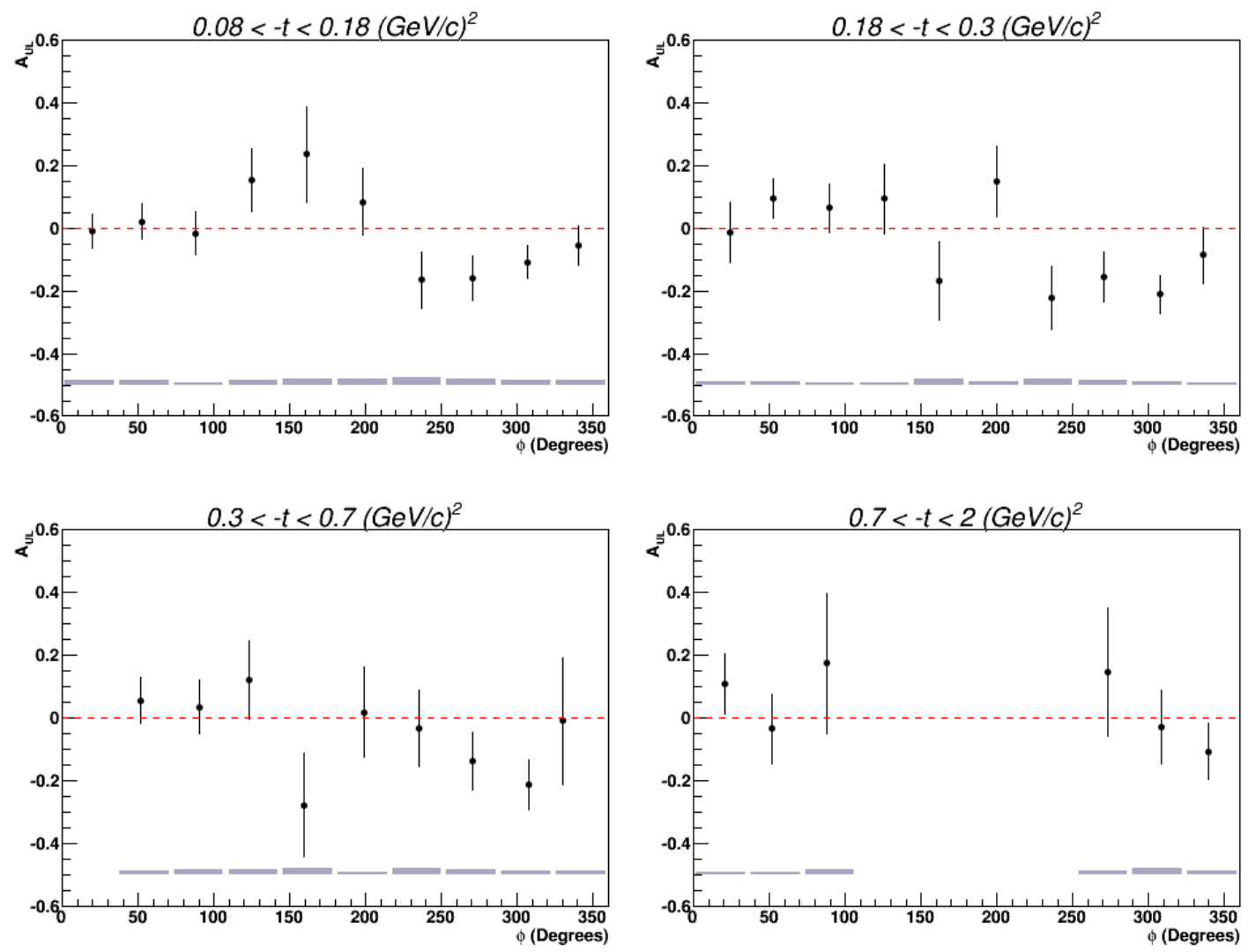

Fig. 5.22: Target-spin asymmetry for DVCS-BH events, as a function of $\phi$, for the various $-t$ bins, for the third bin in $Q^{2}-x_{B}$. The vertical lines indicate the statistical error, and the gray areas indicate the systematic error. 

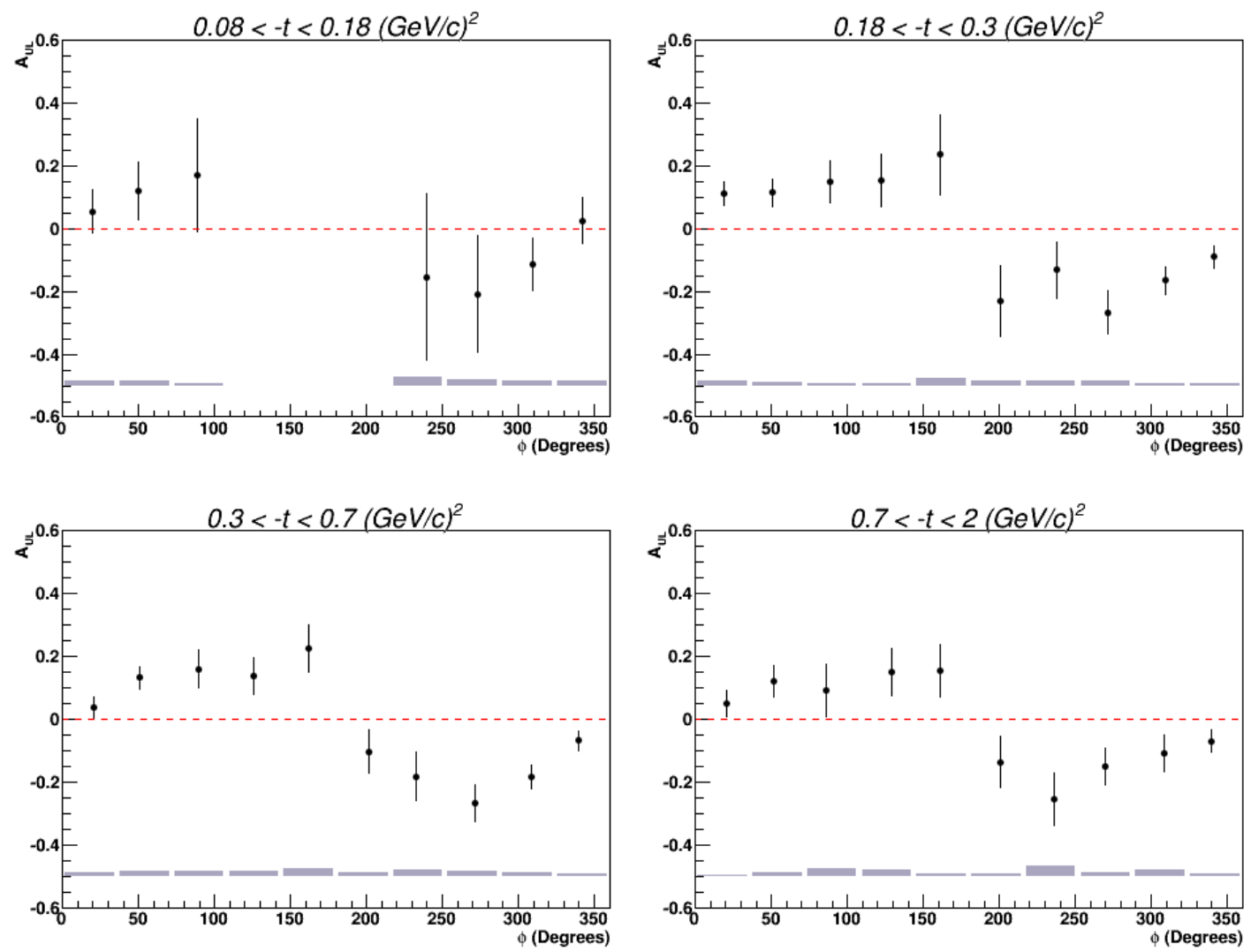

Fig. 5.23: Target-spin asymmetry for DVCS-BH events, as a function of $\phi$, for the various $-t$ bins, for the fourth bin in $Q^{2}-x_{B}$. The vertical lines indicate the statistical error, and the gray areas indicate the systematic error. 

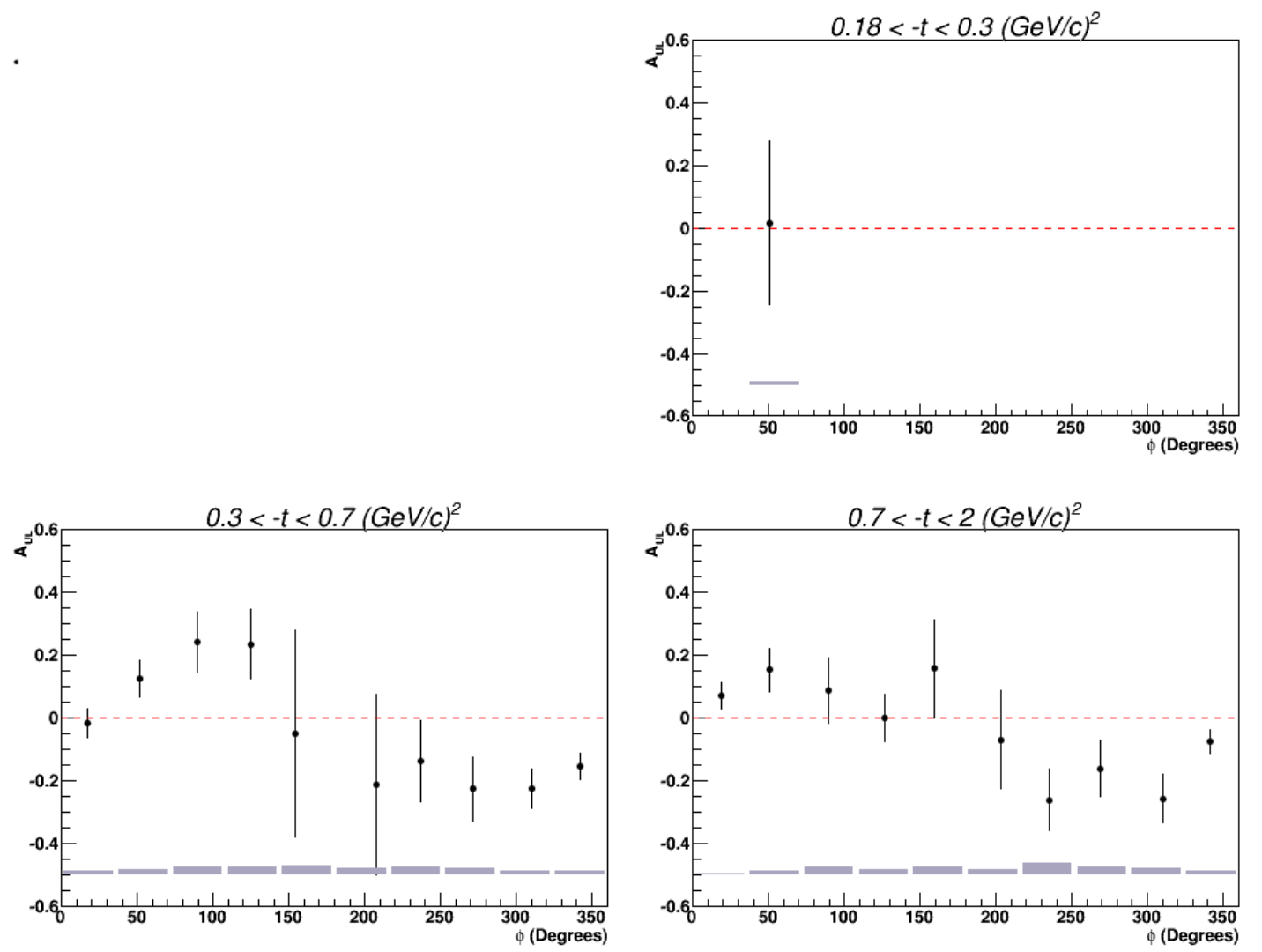

Fig. 5.24: Target-spin asymmetry for DVCS-BH events, as a function of $\phi$, for the various $-t$ bins, for the fifth bin in $Q^{2}-x_{B}$. The vertical lines indicate the statistical error, and the gray areas indicate the systematic error. 


\section{Chapter 6}

\section{Physics Analysis: Extraction of Compton Form Factors}

The method of extraction of Compton Form Factors (CFFs) developed by M. Guidal [3], takes a given experimental kinematic point, and uses the CFFs as free parameters to extract them from DVCS observables using the well-established DVCS + Bethe-Heitler theoretical amplitude. The Bethe-Heitler amplitude is calculated while the DVCS amplitude is taken at the QCD leading twist. The expression of these amplitudes can be found in Reference [52]. As there are eight CFFs (as discussed in Section 1.3) as free parameters, the more DVCS observables are fitted simultaneously (i.e. at the same kinematic point), the more constraints can be put on the fit, and thus the more CFFs can be extracted. The results of the measurement presented in this thesis, the DVCS-BH target-spin asymmetry,

$$
A_{U L}=\frac{N^{\uparrow \Uparrow}+N^{\downarrow \Uparrow}-N^{\uparrow \Downarrow}-N^{\downarrow \Downarrow}}{D_{f}\left(P_{t}^{\Downarrow}\left(N^{\uparrow \Uparrow}+N^{\downarrow \Uparrow}\right)+P_{t}^{\Uparrow}\left(N^{\uparrow \Downarrow}+N^{\downarrow \Downarrow}\right)\right)},
$$

along with the DVCS-BH electron beam-spin asymmetry measured by S. Pisano [1],

$$
A_{L U}=\frac{P_{t}^{\Downarrow}\left(N^{\uparrow \Uparrow}-N^{\downarrow \Uparrow}\right)+P_{t}^{\Uparrow}\left(N^{\uparrow \Downarrow}-N^{\downarrow \Downarrow}\right)}{P_{b}\left(P_{t}^{\Downarrow}\left(N^{\uparrow \Uparrow}+N^{\uparrow \Downarrow}\right)+P_{t}^{\Uparrow}\left(N^{\downarrow \Uparrow}+N^{\downarrow \Downarrow}\right)\right)},
$$

and the DVCS-BH double-spin asymmetry also measured by S. Pisano [1],

$$
A_{L L}=\frac{N^{\uparrow \Uparrow}+N^{\downarrow \Downarrow}-N^{\uparrow \Downarrow}-N^{\downarrow \Uparrow}}{P_{b} \cdot D_{f}\left(P_{t}^{\Downarrow}\left(N^{\uparrow \Uparrow}+N^{\downarrow \uparrow}\right)+P_{t}^{\Uparrow}\left(N^{\uparrow \Downarrow}+N^{\downarrow \Downarrow}\right)\right)} .
$$

extracted from the EG1-DVCS dataset in the same kinematic bins, were provided to Michel Guidal to be used in his fitting procedure to extract Compton Form Factors [3]. An overview of the relationship between the three spin observables and Compton Form Factors are discussed separately in the following Sections (6.1 - 6.2). A complete description of the relation to GPDs can be found in Reference [5]. 


\subsection{Target-Spin Asymmetry}

The single spin asymmetry $A_{U L}$ is given, at twist-2 level, by:

$$
\begin{aligned}
A_{\mathrm{UL}}(\phi) & \sim \frac{x_{B}}{y} \frac{s_{1, \mathrm{LP}}^{\mathcal{I}}}{c_{0, \mathrm{unp}}^{\mathrm{BH}}+\ldots} \sin \phi \\
& \propto \Im \mathrm{m}\left\{F_{1} \widetilde{\mathcal{H}}+\frac{x_{B}}{2-x_{B}}\left(F_{1}+F_{2}\right)\left(\mathcal{H}+\frac{x_{B}}{2} \mathcal{E}\right)+\ldots\right\} \sin \phi,
\end{aligned}
$$

where the dots in the denominator represent known BH terms or smaller interference terms, while the dots in the numerator represent terms proportional to $\widetilde{\mathcal{E}}$, kinematically suppressed by factors of order $x_{B}^{2}$ or $x_{B} t / 4 M^{2}$. So $A_{\mathrm{UL}}(\phi)$ is in first approximation a linear function of CFFs with a dominant contribution from $\Im \mathrm{m} \mathcal{H}$ and $\Im \mathrm{m} \widetilde{\mathcal{H}}$, that is from the GPDs $H$ and $\tilde{H}$ along the lines $x= \pm \xi[42]$.

\subsection{Beam-Spin Asymmetry}

The expression at twist-two of the beam-spin asymmetry is

$$
\begin{aligned}
A_{\mathrm{LU}}(\phi) & \sim \frac{x_{B}}{y} \frac{s_{1, \text { unp }}^{\mathcal{I}}}{c_{0, \text { unp }}^{\mathrm{BH}}+\ldots} \sin \phi \\
& \propto \Im \mathrm{m}\left\{F_{1} \mathcal{H}+\frac{x_{B}}{2-x_{B}}\left(F_{1}+F_{2}\right) \widetilde{\mathcal{H}}-\frac{\Delta^{2}}{4 M^{2}} F_{2} \mathcal{E}+\ldots .\right\} \sin \phi,
\end{aligned}
$$

where $\Delta^{2}$ is the squared momentum transfer between the target proton and the outgoing one.

\subsection{Double-Spin Asymmetry}

Unlike $A_{\mathrm{UL}}$, the Bethe-Heitler process alone can generate a double spin asymmetry $A_{\mathrm{LL}}$. At twist-2 level, this observable takes the form:

$$
\begin{aligned}
A_{\mathrm{LL}}(\phi) & \sim \frac{x_{B}}{y} \frac{c_{0, \mathrm{LP}}^{\mathrm{BH}}+c_{0, \mathrm{LP}}^{\mathcal{I}}+\left(c_{1, \mathrm{LP}}^{\mathrm{BH}}+c_{1, \mathrm{LP}}^{\mathcal{I}}\right) \cos \phi}{c_{0, \mathrm{unp}}^{\mathrm{BH}}+\ldots} \\
\text { with } c_{0, \mathrm{LP}}^{\mathcal{I}} \text { and } c_{1, \mathrm{LP}}^{\mathcal{I}} & \propto \Re \mathrm{e}\left\{F_{1} \widetilde{\mathcal{H}}+\frac{x_{B}}{2-x_{B}}\left(F_{1}+F_{2}\right)\left(\mathcal{H}+\frac{x_{B}}{2} \mathcal{E}\right)+\ldots\right\},
\end{aligned}
$$

In this expression, the interference terms are expected to be smaller, than the known $\mathrm{BH}$ terms. Nonetheless, it is expected that in some parts of the phase space $A_{\mathrm{LL}}$ has a measurable sensitivity to $\Re \mathrm{e} \mathcal{H}$ and $\Re \mathrm{e} \widetilde{\mathcal{H}}$. 


\subsection{Extracted Compton Form Factors}

In M. Guidal's approach, $\Im \mathrm{m} \tilde{\mathcal{E}}$ is set to zero, as $\tilde{E}$ is generally associated with the pion pole t-channel exchange $\left(\left(1 /\left(t-m_{\pi}^{2}\right)\right)\right.$ which is real. Thus seven out of the eight CFFs are left as free parameters in the fit. Figures 6.1-6.5 show the 5 CFFs $(\Im \mathrm{m} \mathcal{H}, \Im \mathrm{m} \tilde{\mathcal{H}}, \Re \mathrm{H}, \Re$, $\tilde{\mathcal{H}}$, $\Im m \mathcal{E}$ ) for which presentable results had been obtained, as a function of $-t$ and for each of the $5 Q^{2}-x_{B}$ bins discussed in Section 3.7. In each plot, the fitted value (blue point with error bars) is shown along with the prediction of the VGG model (red square). Only the CFFs in bins for which the fit gave a result with non-zero error bars are plotted. The results for $\Im \mathrm{m} \mathcal{H}$ and $\Im \mathrm{m} \tilde{\mathcal{H}}$ (Figs. 6.1 and 6.2 ) show that the $t$-slope $^{1}$ of $\Im \mathrm{m} \mathcal{H}$ is much stronger than that of $\Im \mathrm{m} \tilde{\mathcal{H}}$, hinting to the fact that the axial charge (linked to $\Im$ m $\tilde{\mathcal{H}}$ ) might be more "concentrated" in the center of the nucleon than the electromagnetic charge (linked to $\Im m \mathcal{H}$ ). The three observables displayed some sensitivity to the GPD $E$, as is shown in Fig. 6.5. There, $\Im m \mathcal{E}$ appears to be much smaller than $\Im m \mathcal{H}$, in agreement with what the VGG model predicts, and with shallower $t$ dependency.

\footnotetext{
${ }^{1} t$ is the Fourier transform of the transverse position of the parton in the coordinates space.
} 

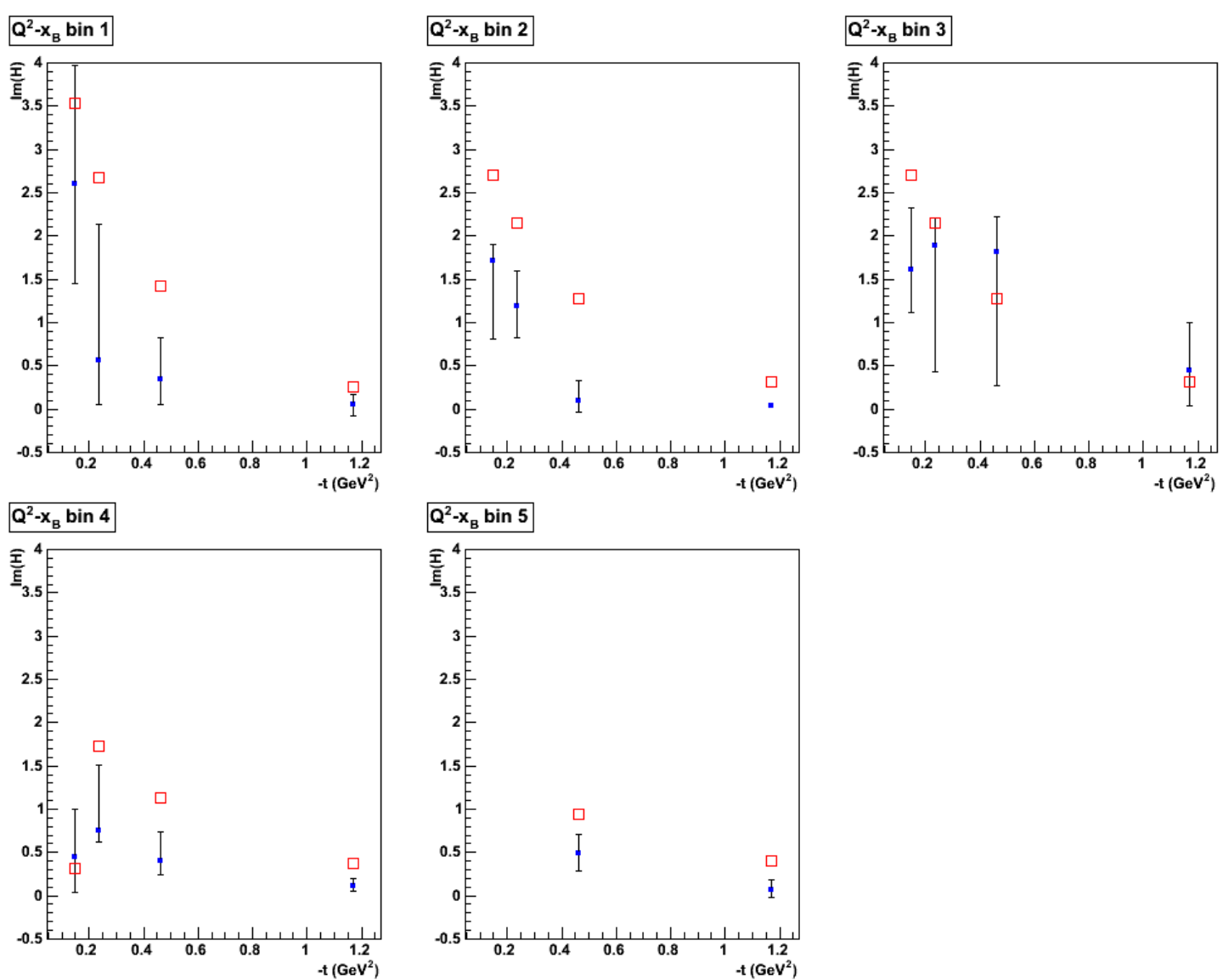

Fig. 6.1: $\Im m \mathcal{H}$, as a function of $-t$, for each of our $5 Q^{2}-x_{B}$ bins. The blue points are the results of the fit to the EG1-DVCS data, the red squares are the predictions of the VGG model. 

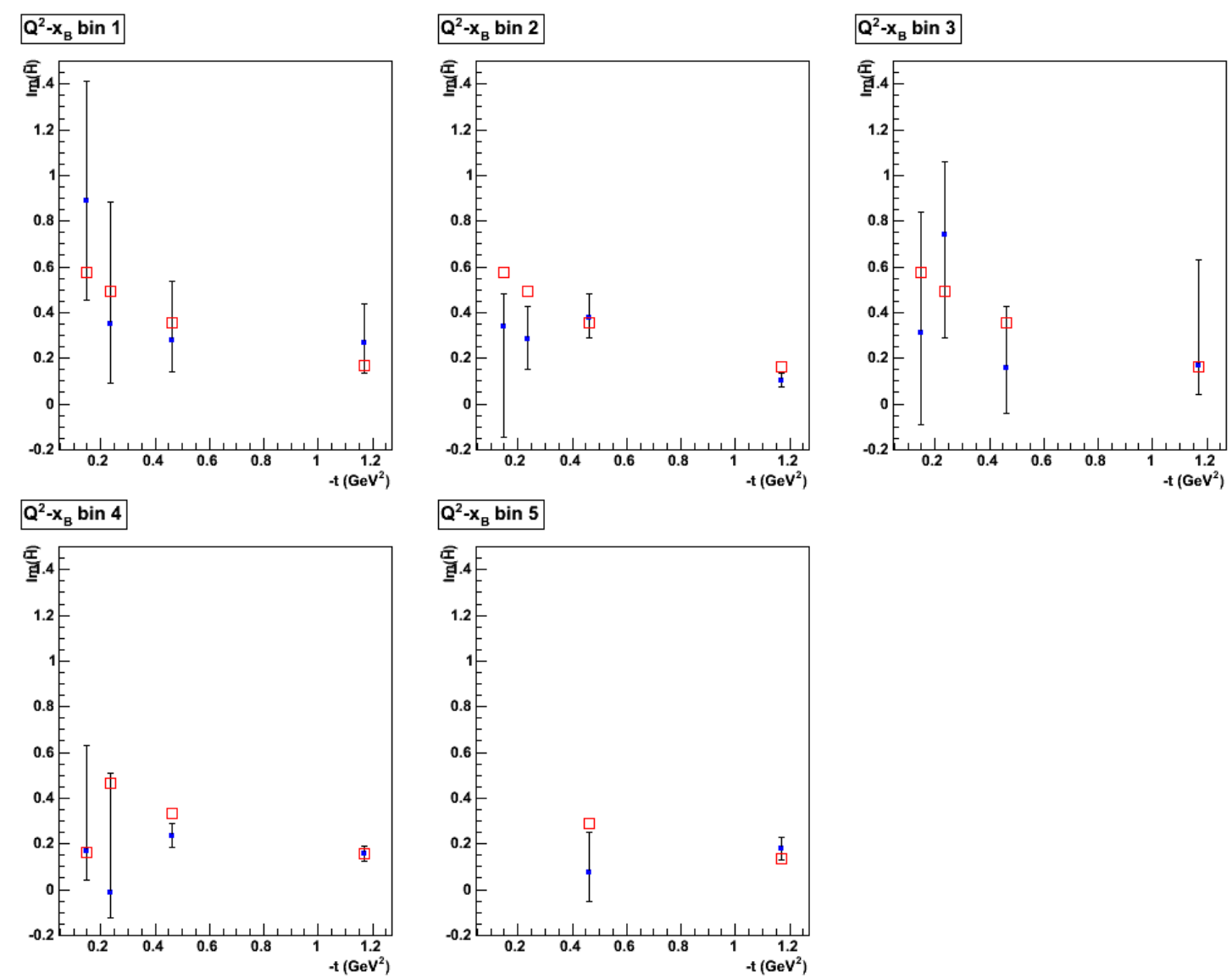

Fig. 6.2: $\Im \mathrm{m} \tilde{\mathcal{H}}$, as a function of $-t$, for each of our $5 Q^{2}-x_{B}$ bins. The blue points are the results of the fit to the EG1-DVCS data, the red squares are the predictions of the VGG model. 

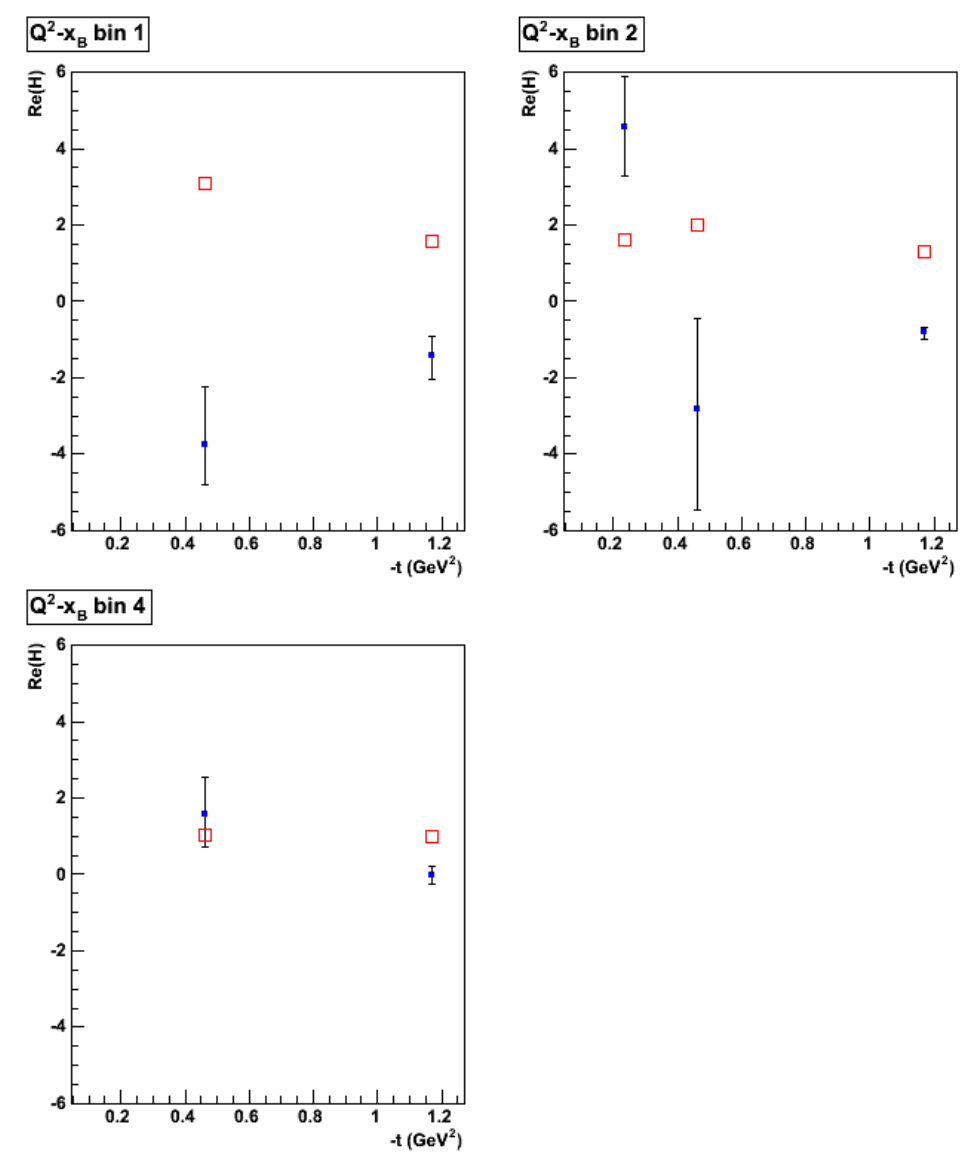

Fig. 6.3: $\Re \mathrm{e} \mathcal{H}$, as a function of $-t$, for three of our $5 Q^{2}-x_{B}$ bins. The blue points are the results of the fit to the EG1-DVCS data, the red squares are the predictions of the VGG model. 

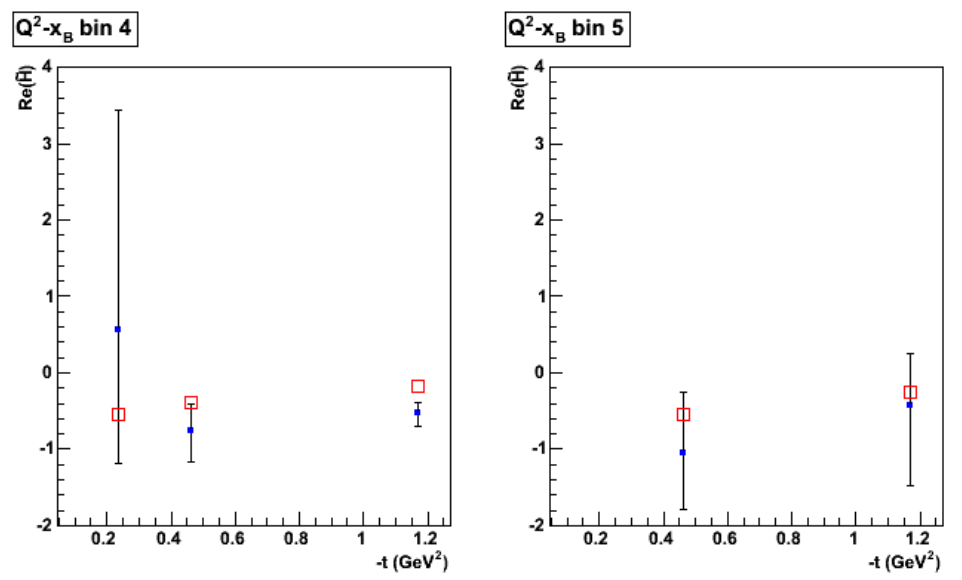

Fig. 6.4: $\Re$ e $\tilde{\mathcal{H}}$, as a function of $-t$, for two of our $5 Q^{2}-x_{B}$ bins. The blue points are the results of the fit to the EG1-DVCS data, the red squares are the predictions of the VGG model. 

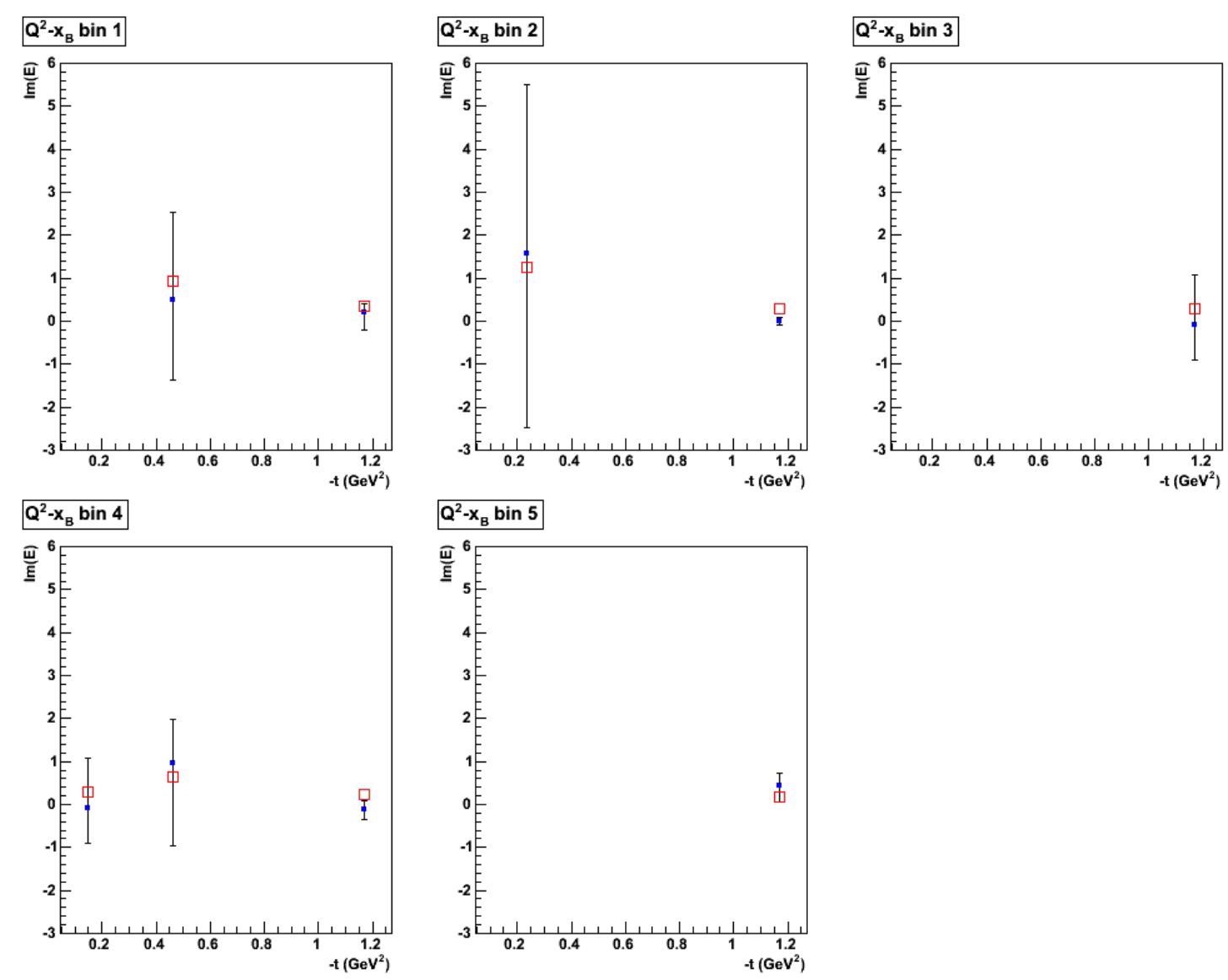

Fig. 6.5: $\Im m \mathcal{E}$, as a function of $-t$, for each of our $5 Q^{2}-x_{B}$ bins. The blue points are the results of the fit to the EG1-DVCS data, the red squares are the predictions of the VGG model. 


\section{Chapter 7}

\section{Conclusions}

Target-spin asymmetries for Deeply Virtual Compton Scattering were measured from the EG1-DVCS experiment data set. This experiment used the CLAS detector in conjunction with an additional Inner Calorimeter and the Hall B longitudinally-polarized $\mathrm{NH}_{3}$ target in 2004 -dimensional bins in $Q^{2}, x_{B},-t$ and $\phi$, covering a wide kinematic range $\left(1<Q^{2}<5\right.$ $\left.\mathrm{GeV}^{2}, 0.12<x_{B}<0.6,0.08<-t<2 \mathrm{GeV}^{2}, 0<\phi<360^{\circ}\right)$. The $\phi$ dependence of the obtained asymmetries was studied. The prevalence of the $\sin \phi$ term in the target-spin asymmetries was consistent with a dominance of the handbag mechanism. The predictions of the VGG model are in qualitative agreement with the measurements. The simultaneous measurement of three DVCS spin observables, $A_{U L}$ (this work), $A_{L U}$ and $A_{L L}$ (measured by S. Pisano [1]), at the same kinematic points allowed for a semi-model-independent extraction of

5 Compton Form Factors, among which $\Im \mathrm{m} \mathcal{H}$ and $\Im \mathrm{m} \tilde{\mathcal{H}}$ display the best statistical precision and kinematic coverage. 


\section{Appendix A}

\section{DVCS Channel Selection - Run Period A}
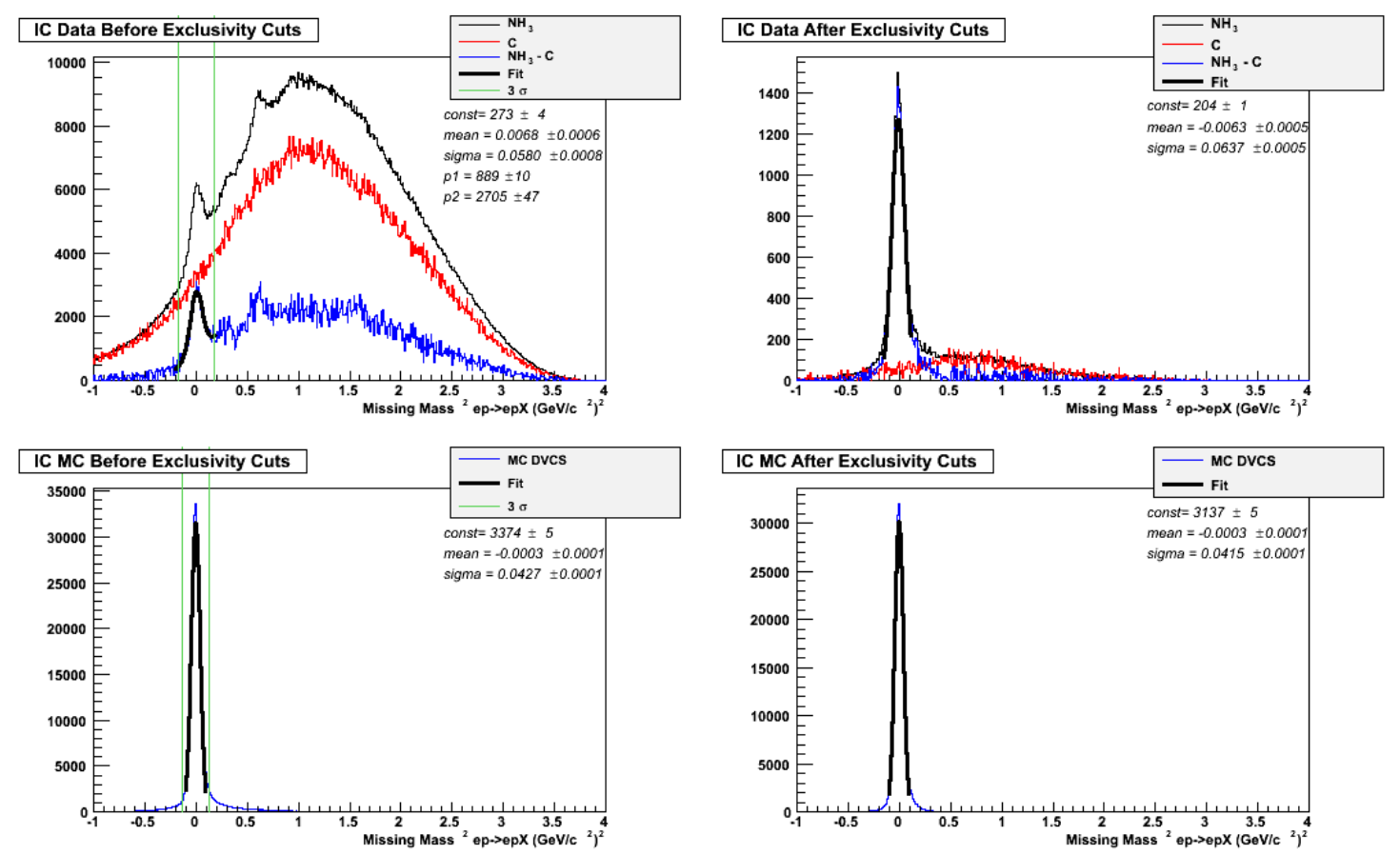

Fig. A.1: DVCS selection Run Period A, IC topology Missing mass of the ep system. Top left: without any but the preliminary "DIS" and $E_{\gamma}$ cuts applied for $\mathrm{NH}_{3}$ data (black), carbon data (red), $\mathrm{NH}_{3}$ data after carbon subtraction (blue); the thick black line is a Gaussian-plus-linear-background fit to the blue curve; the $\pm 3 \sigma$ cut window is indicated by the two green vertical lines. Top right, after all exclusivity cuts, except the one on $M M^{2}(e p)$, have been applied, same color code as the top-left plot. Bottom left, DVCS/Bethe-Heitler Monte-Carlo simulation, without any but the preliminary "DIS" and $E_{\gamma}$ cuts, fitted with a Gaussian curve (black thick line). Bottom right: DVCS/Bethe-Heitler Monte-Carlo simulation, after all exclusivity cuts except the one on $M M^{2}(e p)$ have been applied, fitted with a Gaussian curve (black thick line). 

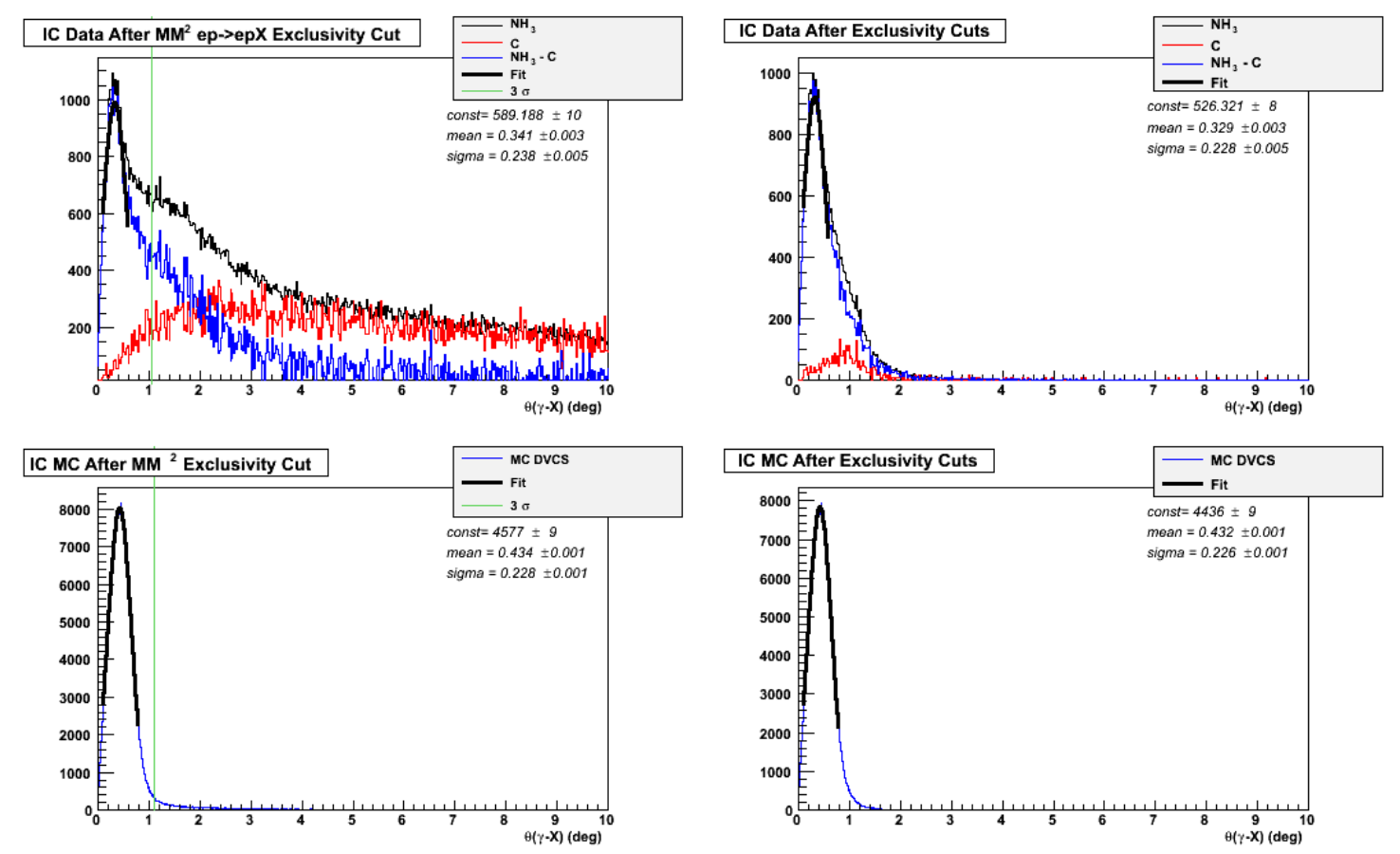

Fig. A.2: DVCS selection Run Period A, IC topology $\theta_{\gamma X}$ distributions. Top left, with "DIS" and $E_{\gamma}$ cuts plus a $3 \sigma$ cut on $M M^{2}(e p)$ applied for $N H_{3}$ data (black), carbon data (red), $\mathrm{NH}_{3}$ data after carbon subtraction (blue); the thick black line is a Gaussian fit to the blue curve, the $\pm 3 \sigma$-cut window is indicated by the two green vertical lines. Top right, after all exclusivity cuts, except the one on $\theta_{\gamma X}$, have been applied, same color code as the top-left plot. Bottom left, DVCS/Bethe-Heitler Monte-Carlo simulation, without any but the preliminary "DIS" and $E_{\gamma}$ cuts plus a $3 \sigma$ cut on $M M^{2}(e p)$, fitted with a Gaussian curve (black thick line). Bottom right: DVCS/Bethe-Heitler Monte-Carlo simulation, after all exclusivity cuts except the one on $\theta_{\gamma X}$ have been applied, fitted with a Gaussian curve (black thick line). 

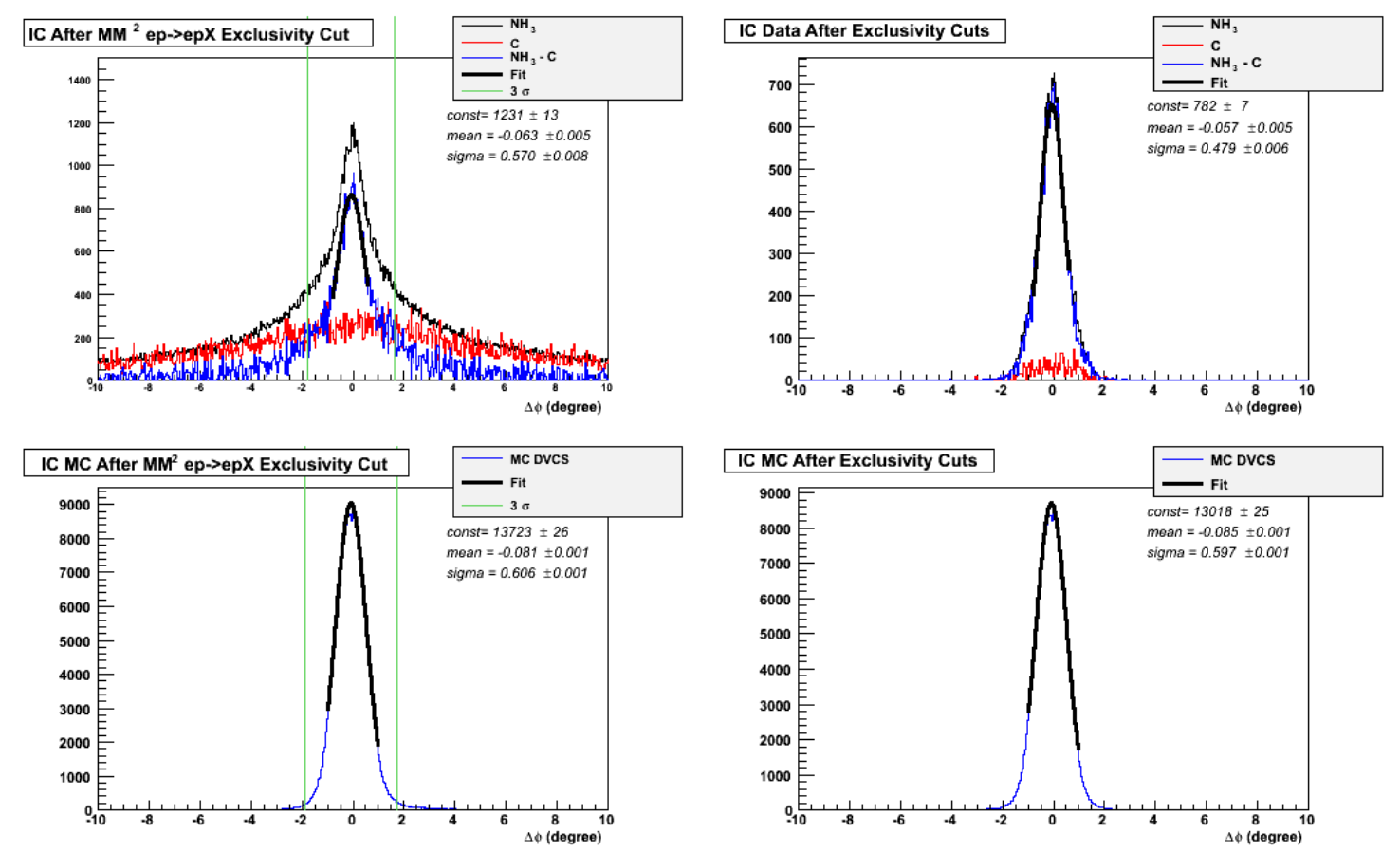

Fig. A.3: DVCS selection Run Period A, IC topology $\Delta \phi$ distributions. Top left, with "DIS" and $E_{\gamma}$ cuts plus a $3 \sigma$ cut on $M M^{2}(e p)$ applied for $N H_{3}$ data (black), carbon data (red), $\mathrm{NH}_{3}$ data after carbon subtraction (blue); the thick black line is a Gaussian fit to the blue curve, the $\pm 3 \sigma$-cut window is indicated by the two green vertical lines. Top right, after all exclusivity cuts except the one on $\Delta \phi$ have been applied, same color code as the top-left plot. Bottom left, DVCS/BetheHeitler Monte-Carlo simulation, without any but the preliminary "DIS" and $E_{\gamma}$ cuts plus a $3 \sigma$ cut on $M M^{2}(e p)$, fitted with a Gaussian curve (black thick line). Bottom right: DVCS/Bethe-Heitler Monte-Carlo simulation, after all exclusivity cuts except the one on $\Delta \phi$ have been applied, fitted with a Gaussian curve (black thick line). 

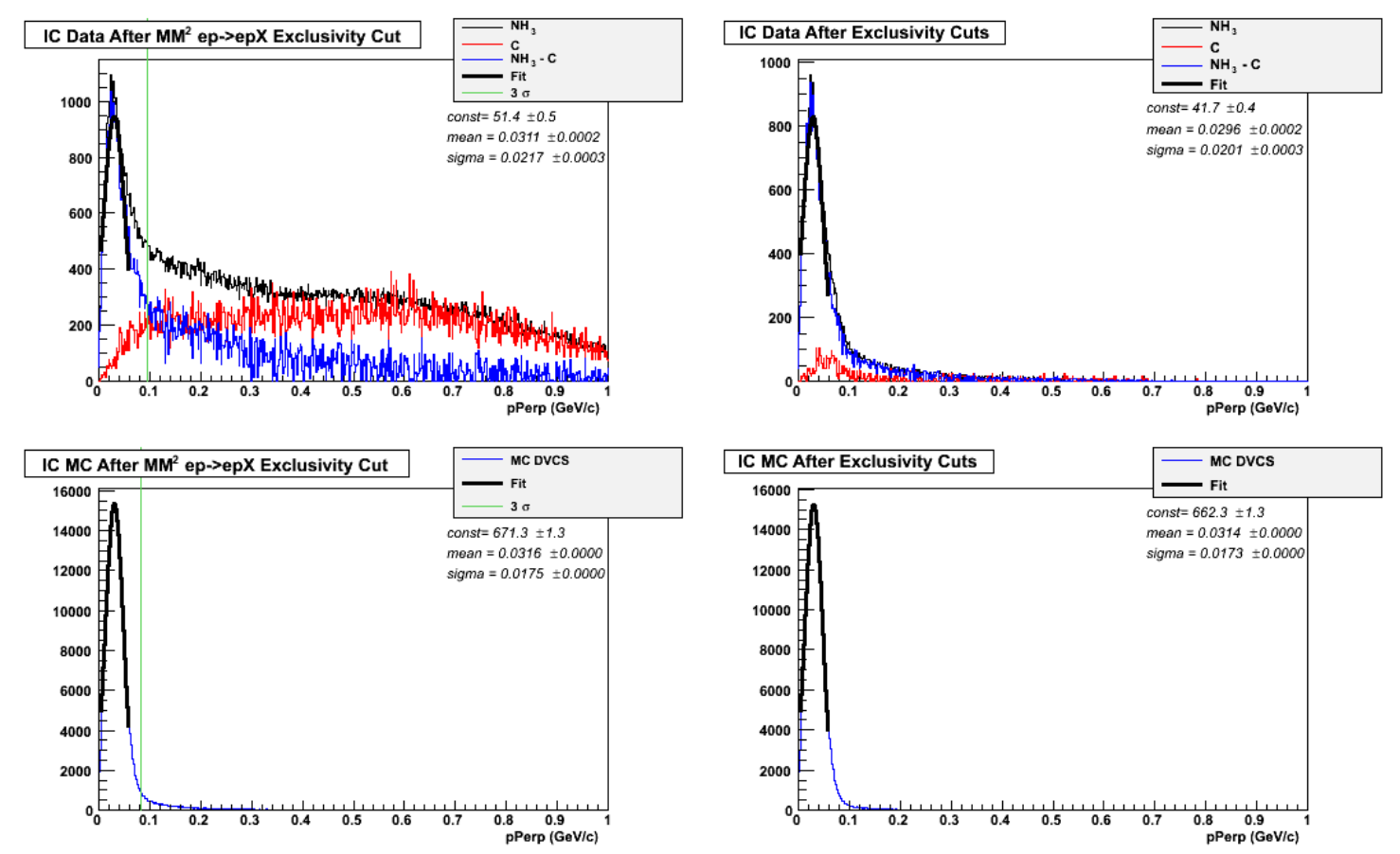

Fig. A.4: DVCS selection Run Period A, IC topology $p_{\text {perp }}$ distributions. Top left, with "DIS" and $E_{\gamma}$ cuts plus a $3 \sigma$ cut on $M M^{2}(e p)$ applied for $N H_{3}$ data (black), carbon data (red), $\mathrm{NH}_{3}$ data after carbon subtraction (blue); the thick black line is a Gaussian fit to the blue curve, the $\pm 3 \sigma$ cut window is indicated by the two green vertical lines. Top right, after all exclusivity cuts except the one on $p_{\text {perp }}$ have been applied, same color code as the top-left plot. Bottom left, DVCS/Bethe-Heitler Monte-Carlo simulation, without any but the preliminary "DIS" and $E_{\gamma}$ cuts plus a $3 \sigma$ cut on $M M^{2}(e p)$, fitted with a Gaussian curve (black thick line). Bottom right: DVCS/Bethe-Heitler Monte-Carlo simulation, after all exclusivity cuts except the one on $p_{\text {perp }}$ have been applied, fitted with a Gaussian curve (black thick line). 

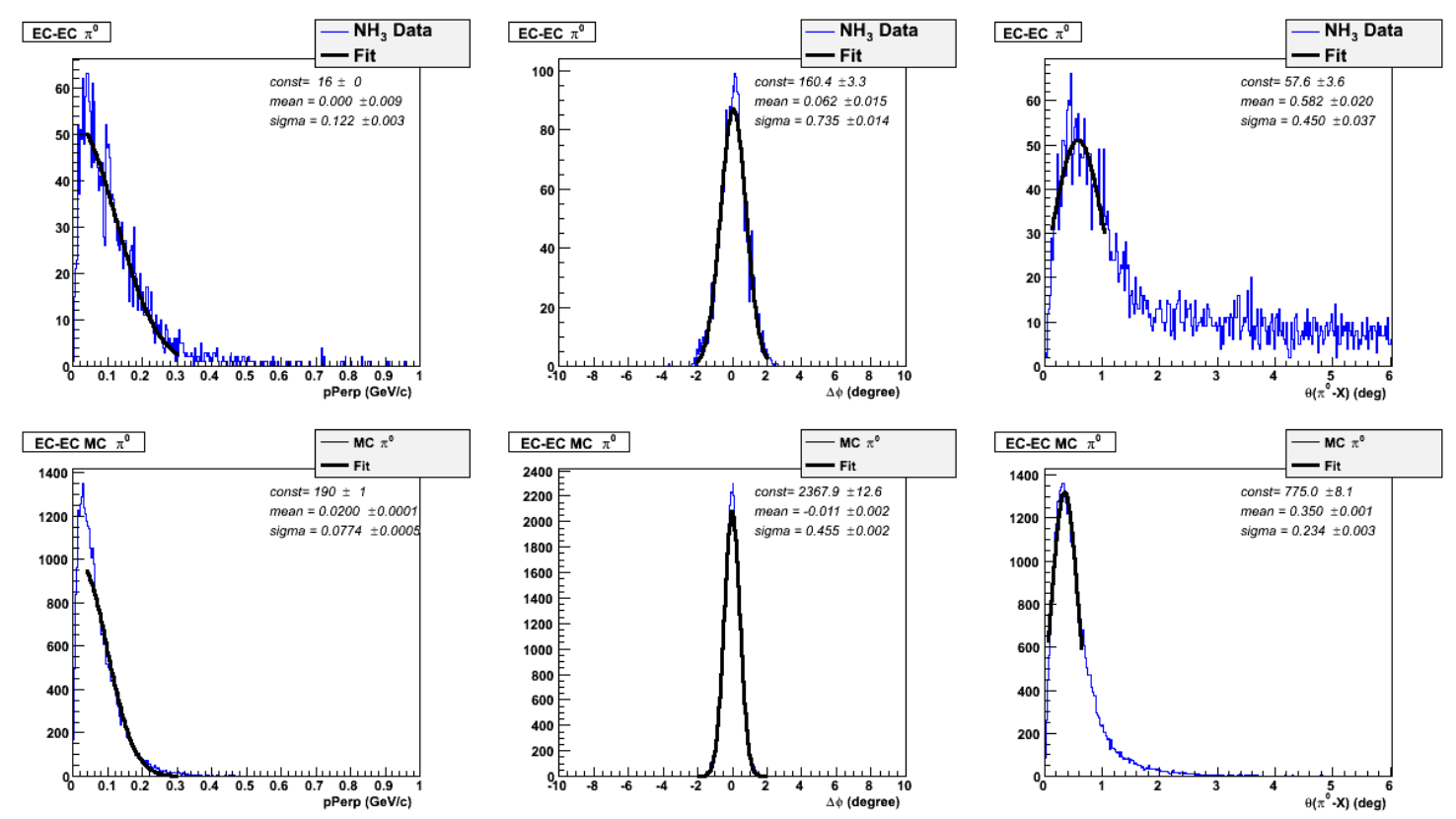

Fig. A.5: $e p \pi^{0}$ analysis Run Period A, EC-EC topology. Top plots: data, after $e p \pi^{0}$ exclusivity cuts; bottom plots: MC. From left to right: $p_{\text {perp }}$ (fit with a half Gaussian), $\Delta \phi$ and $\theta_{\gamma X}$ (both fit with Gaussians). The ratios of the $\sigma$ 's of the top plots over the ones of the bottom ones gives the factor $r_{1}$.
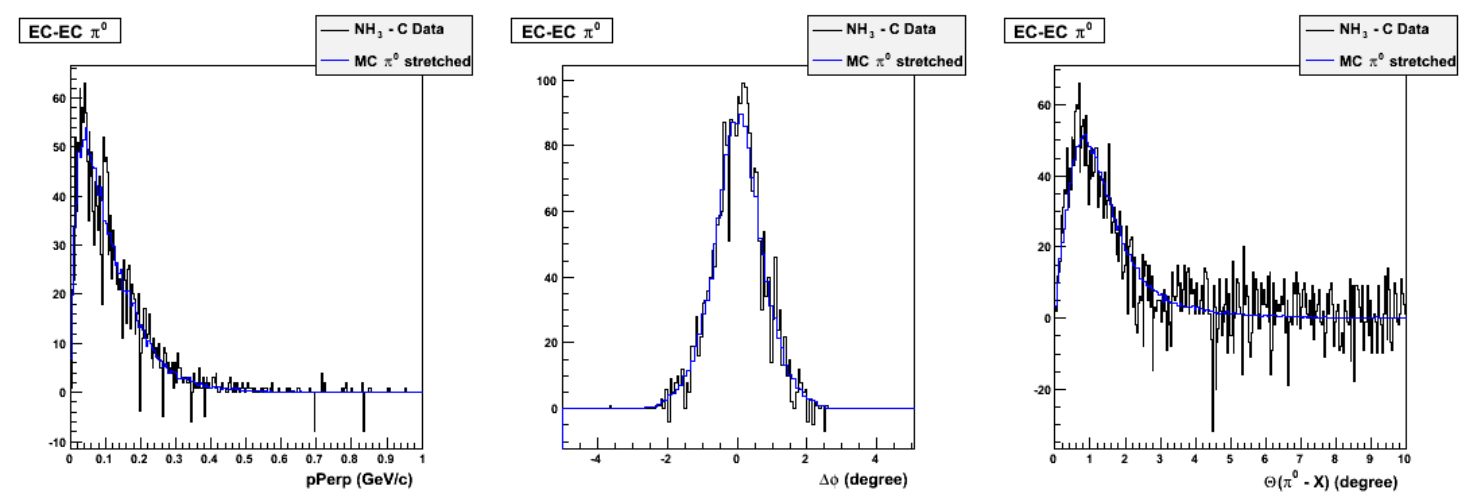

Fig. A.6: $e p \pi^{0}$ analysis Run Period A, EC-EC topology. From left to right: $p_{p e r p}, \Delta \phi$ and $\theta_{\gamma X}$. The data are in blue, the Monte-Carlo, "stretched" by the $r_{1}$ factor, is in green. 

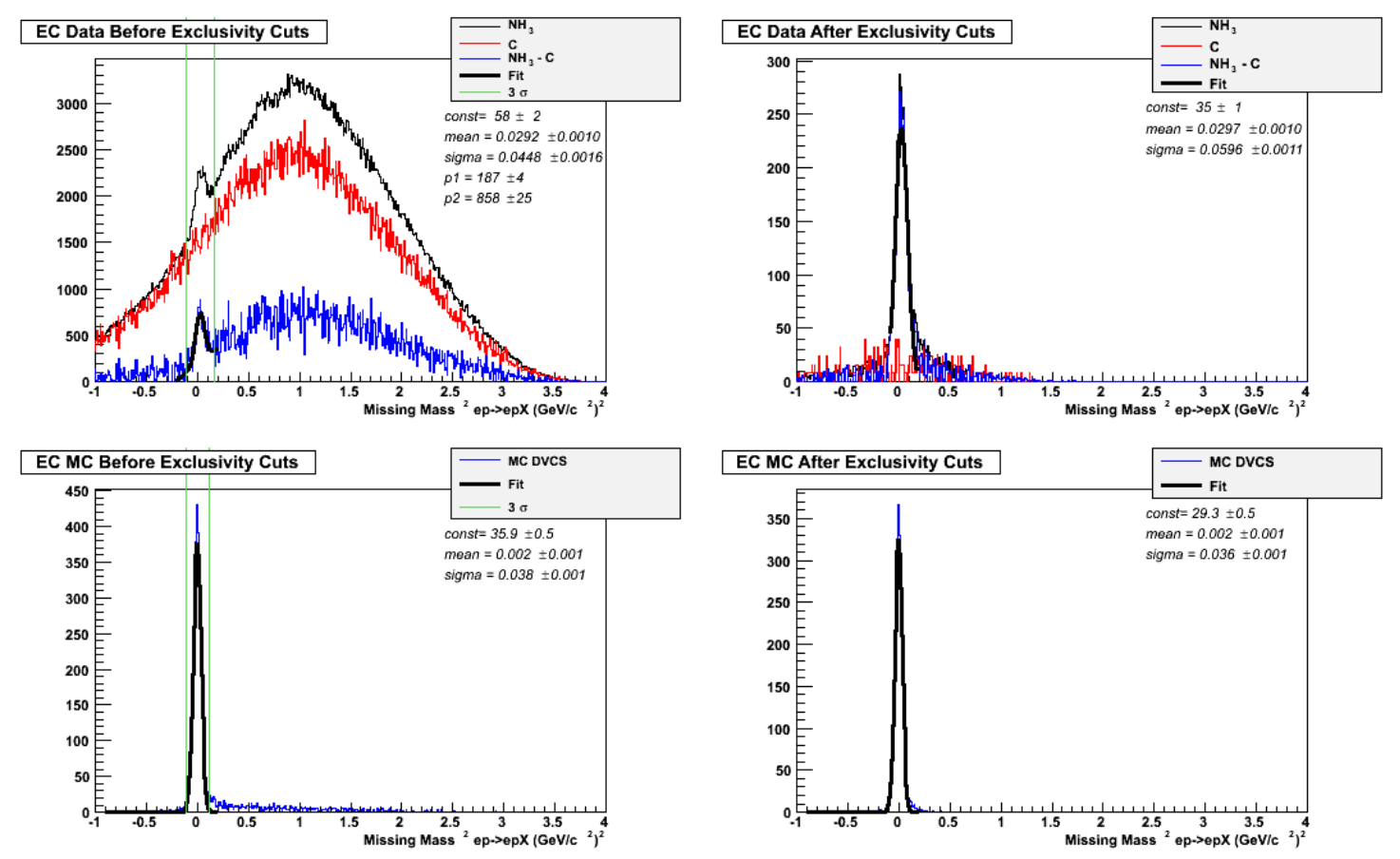

Fig. A.7: DVCS selection Run Period A, EC topology Missing mass of the ep system. Top left, without any but the preliminary "DIS" and $E_{\gamma}$ cuts applied for $\mathrm{NH}_{3}$ data (black), carbon data (red), $\mathrm{NH}_{3}$ data after carbon subtraction (blue); the thick black line is a Gaussian fit to the blue curve, the $\pm 3 \sigma$-cut window is indicated by the two green vertical lines. Top right, after all exclusivity cuts, except the one on $M M^{2}(e p)$, have been applied, same color code as the top-left plot. Bottom left, DVCS/Bethe-Heitler Monte-Carlo simulation, without any but the preliminary "DIS" and $E_{\gamma}$ cuts, fitted with a Gaussian curve (black thick line). Bottom right: DVCS/Bethe-Heitler Monte-Carlo simulation, after all exclusivity cuts except the one on $M M^{2}(e p)$ have been applied, fitted with a Gaussian curve (black thick line). 

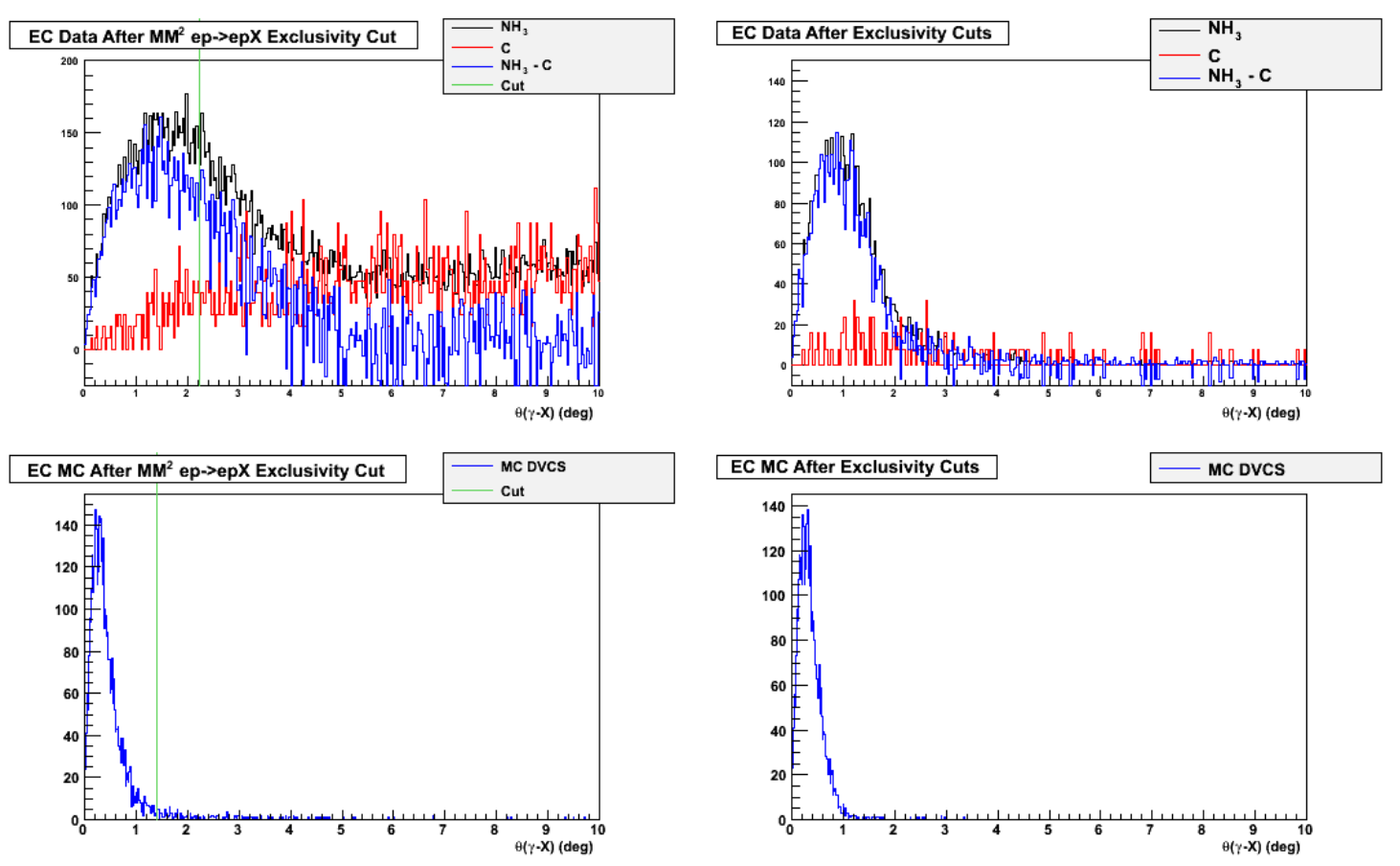

Fig. A.8: DVCS selection Run Period A, EC topology $\theta_{\gamma X}$ distributions. Top left, with "DIS" and $E_{\gamma}$ cuts plus a $3 \sigma$ cut on $M M^{2}(e p)$ applied for $\mathrm{NH}_{3}$ data (black), carbon data (red), $\mathrm{NH}_{3}$ data after carbon subtraction (blue); the green line shows the chosen position of the cut $\left(r_{1} \cdot \sigma_{M C}\right)$. Top right, after all exclusivity cuts, except the one on $\theta_{\gamma X}$, have been applied, same color code as the topleft plot. Bottom left, DVCS/Bethe-Heitler Monte-Carlo simulation, without cuts; the green line shows the chosen position of the cut $\left(\sigma_{M C}\right)$. Bottom right: DVCS/Bethe-Heitler Monte-Carlo simulation, after all exclusivity cuts except the one on $\theta_{\gamma X}$ have been applied. 

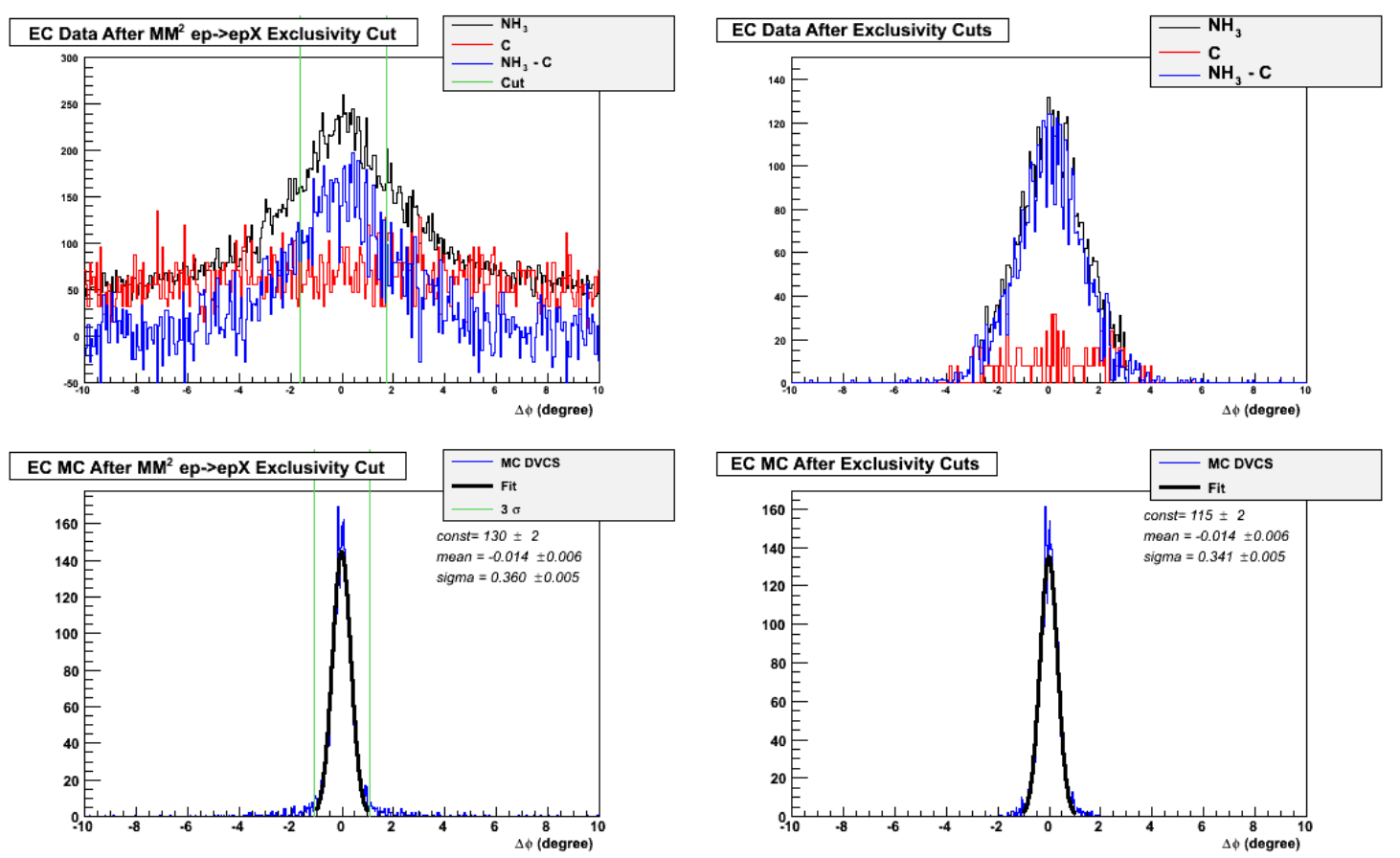

Fig. A.9: DVCS selection Run Period A, EC topology $\Delta \phi$ distributions. Top left, with "DIS" and $E_{\gamma}$ cuts plus a $3 \sigma$ cut on $M M^{2}(e p)$ applied for $\mathrm{NH}_{3}$ data (black), carbon data (red), $\mathrm{NH}_{3}$ data after carbon subtraction (blue); the green lines show the position of the cut $\left( \pm 3 r_{1} \cdot \sigma_{M C}\right)$. Top right, after all exclusivity cuts except the one on $\Delta \phi$ have been applied, same color code as the top-left plot. Bottom left, DVCS/Bethe-Heitler Monte-Carlo simulation, without cuts, fitted with a Gaussian curve (black thick line); the green lines show the position of the cut $\left( \pm 3 \sigma_{M C}\right)$. Bottom right: DVCS/Bethe-Heitler Monte-Carlo simulation, after all exclusivity cuts except the one on $\Delta \phi$ have been applied, fitted with a Gaussian curve (black thick line). 

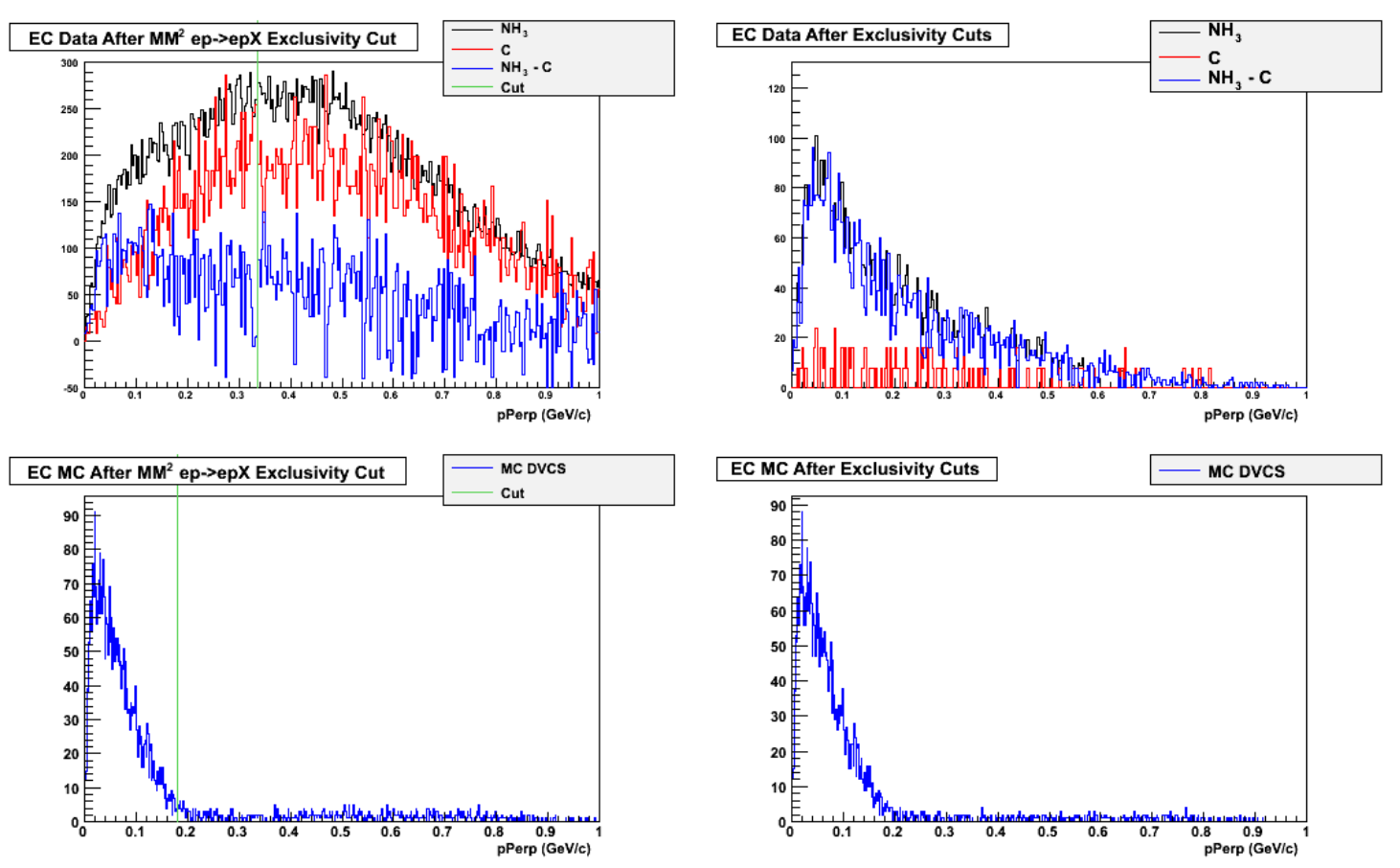

Fig. A.10: DVCS selection Run Period A, EC topology $p_{\text {perp }}$ distributions. Top left, with "DIS" and $E_{\gamma}$ cuts plus a $3 \sigma$ cut on $M M^{2}(e p)$ applied for $\mathrm{NH}_{3}$ data (black), carbon data (red), $\mathrm{NH}_{3}$ data after carbon subtraction (blue)); the green line shows the chosen position of the cut $\left(r_{1} \cdot \sigma_{M C}\right)$. Top right, after all exclusivity cuts except the one on $p_{\text {perp }}$ have been applied. Same color code as the topleft plot. Bottom left, DVCS/Bethe-Heitler Monte-Carlo simulation, without cuts); the green line shows the chosen position of the cut $\left(\sigma_{M C}\right)$. Bottom right: DVCS/Bethe-Heitler Monte-Carlo simulation, after all exclusivity cuts except the one on $p_{\text {perp }}$ have been applied. 


\section{Appendix B}

\section{Exclusive $e p \pi^{0}$ Selection - Run Period A}

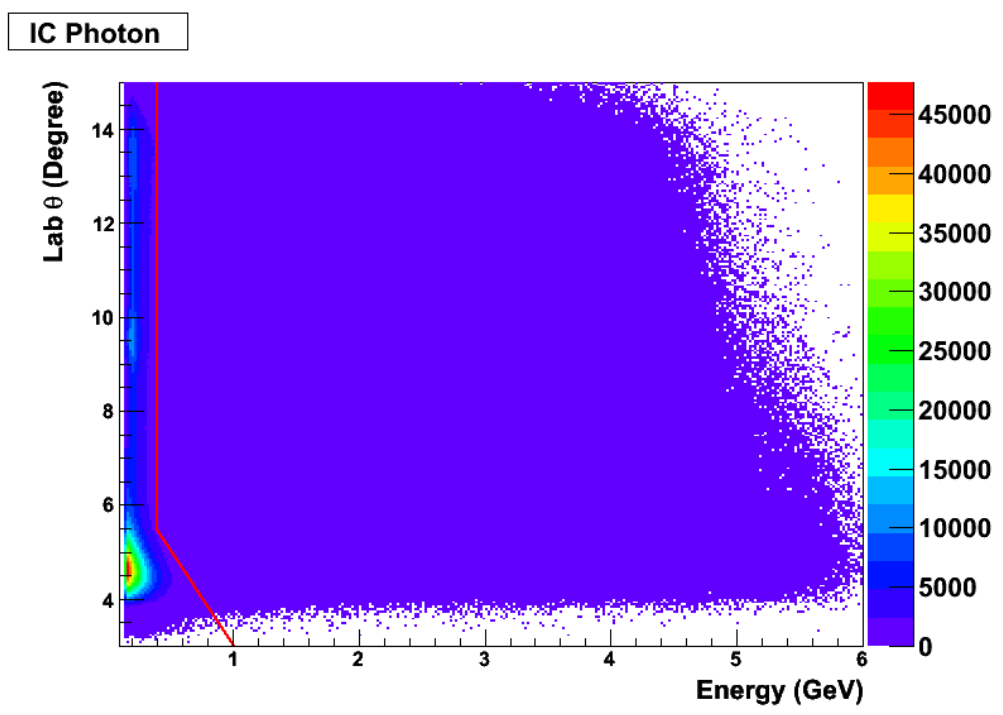

Fig. B.1: Run Period A: Polar angle $\theta$ as a function of the energy for IC hits, showing the cut on the minimum energy at $0.25 \mathrm{GeV}$, as well as the "triangular" cut to remove the low-energy/low- $\theta$ noise, applied to select photons. 

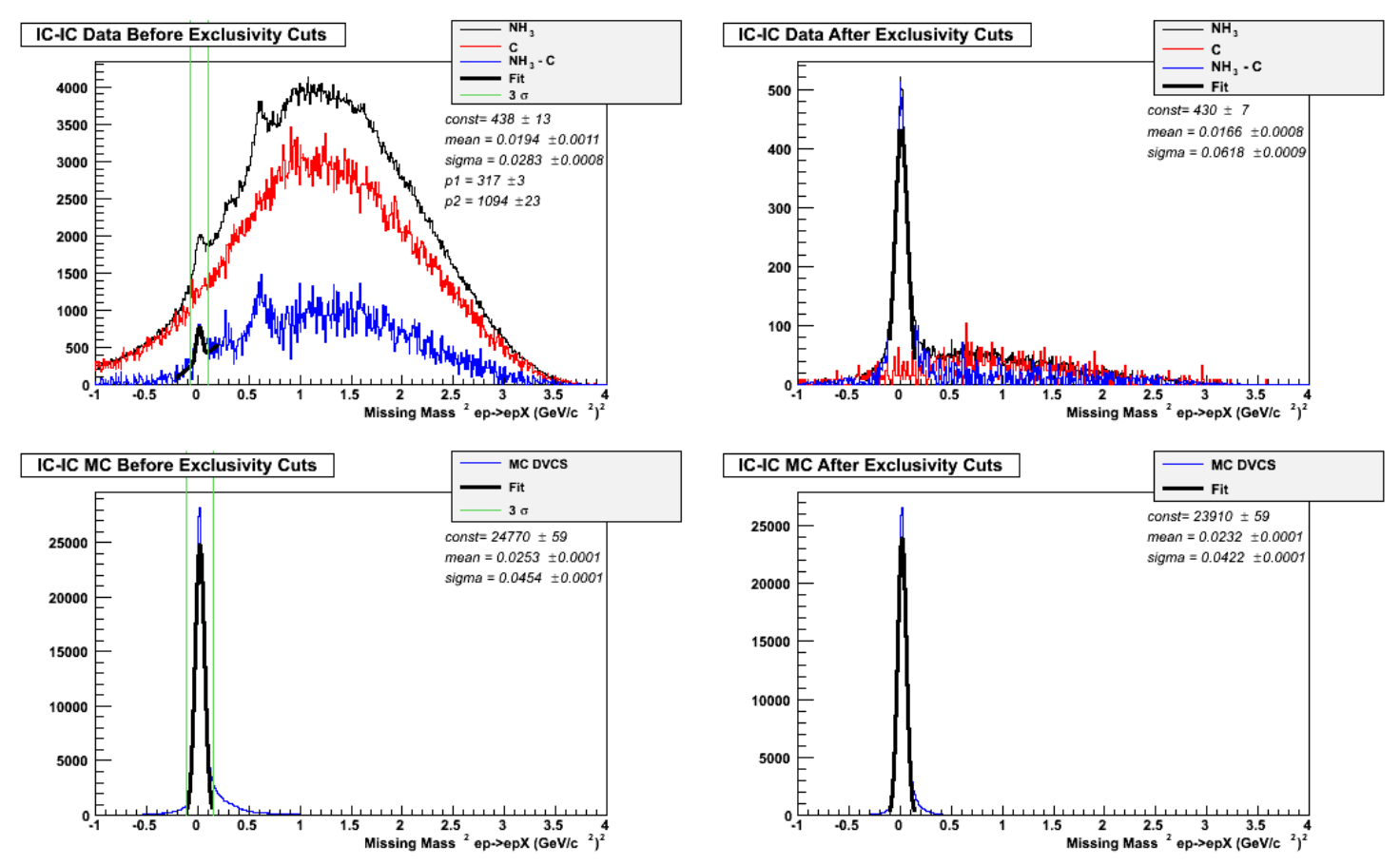

Fig. B.2: Exclusive $\pi^{0}$ analysis Run Period A, IC-IC topology $M M^{2}(e p)$. Top plots: data (black: all $\mathrm{NH}_{3}$ data, red: charge scaled carbon data, blue: $\mathrm{NH}_{3}$ after carbon subtraction). Bottom plots: Monte-Carlo simulation for $e p \pi^{0}$. Left plots: without any but the DIS cuts; right plots: with all exclusivity cuts applied but the one on $M M^{2}(e p)$. 

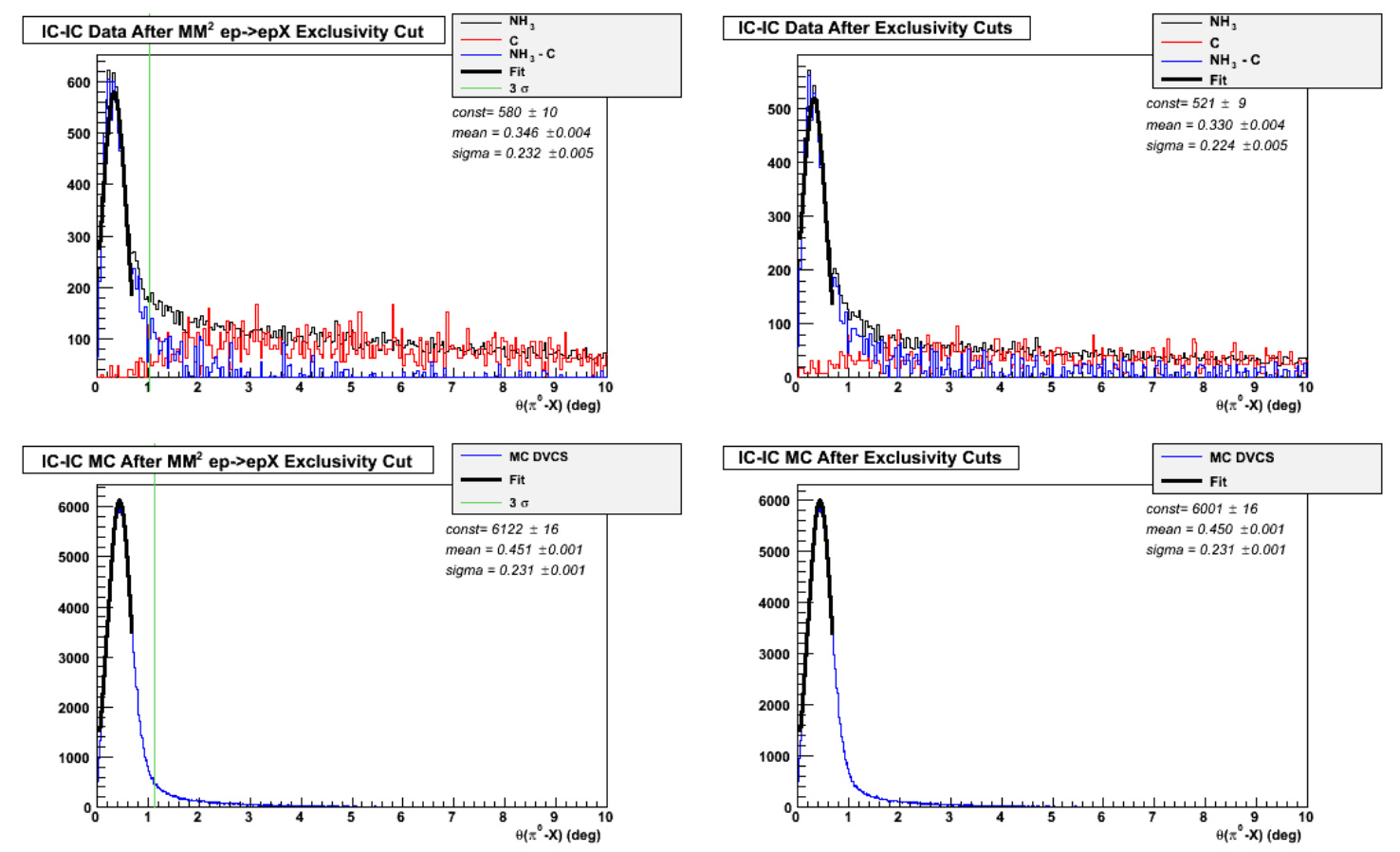

Fig. B.3: Exclusive $\pi^{0}$ analysis Run Period A, IC-IC topology $\theta_{\pi^{0} X}$. Top plots: data (black: all $\mathrm{NH}_{3}$ data, red: charge scaled carbon data, blue: $\mathrm{NH}_{3}$ after carbon subtraction); bottom plots: Monte-Carlo simulation for $e p \pi^{0}$. Left plots: without any but the DIS and the $M M^{2}(e p)$ cuts; right plots: with all exclusivity cuts applied but the one on $\theta_{\pi^{0} X}$. 

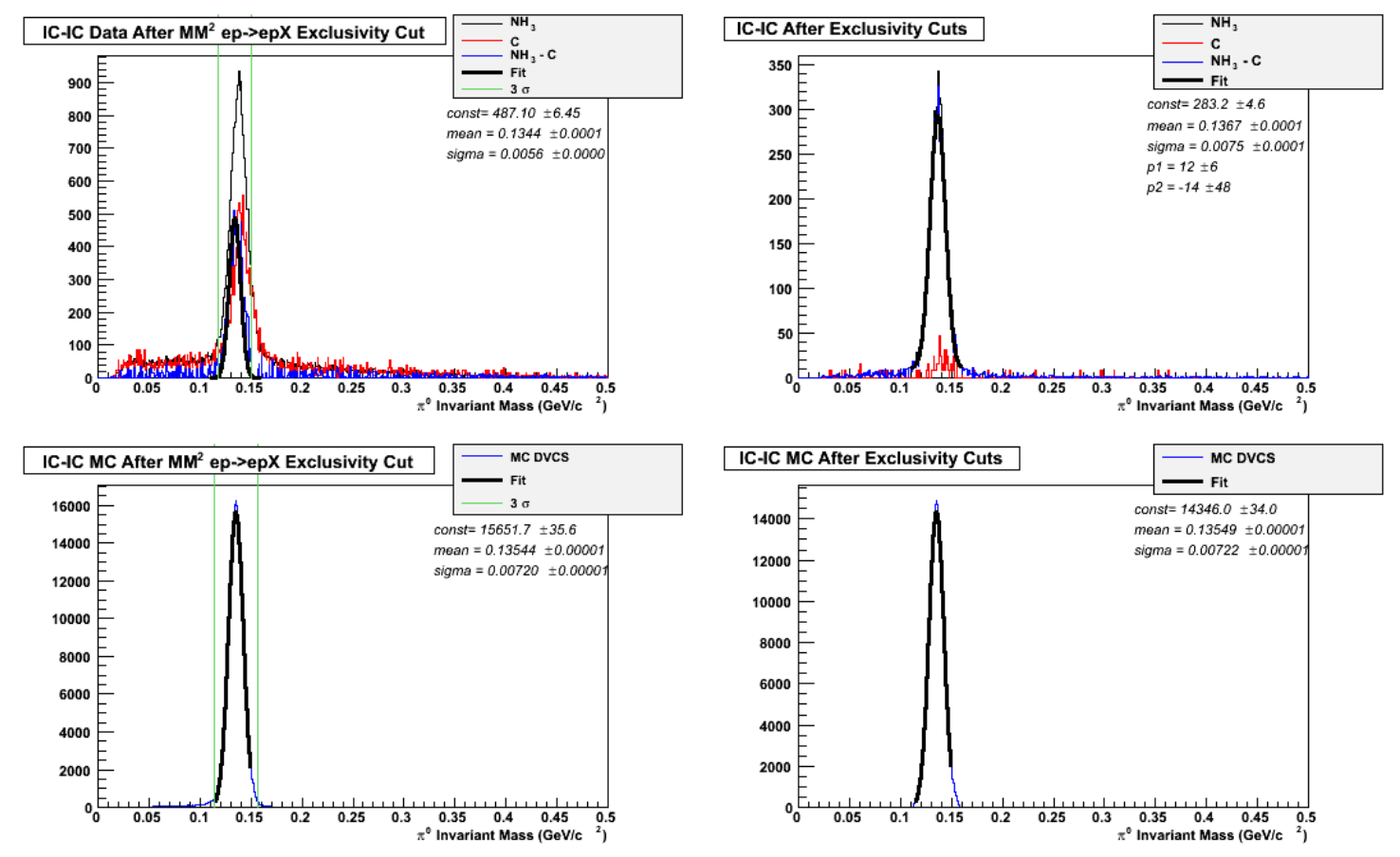

Fig. B.4: Exclusive $\pi^{0}$ analysis Run Period A, IC-IC topology two-photons invariant mass $M(\gamma \gamma)$. Top plots: data (black: all $\mathrm{NH}_{3}$ data, red: charge scaled carbon data, blue: $\mathrm{NH}_{3}$ after carbon subtraction); bottom plots: Monte-Carlo simulation for $e p \pi^{0}$. Left plots: without any but the DIS and the $M M^{2}(e p)$ cuts; right plots: with all exclusivity cuts applied but the one on $M(\gamma \gamma)$. 

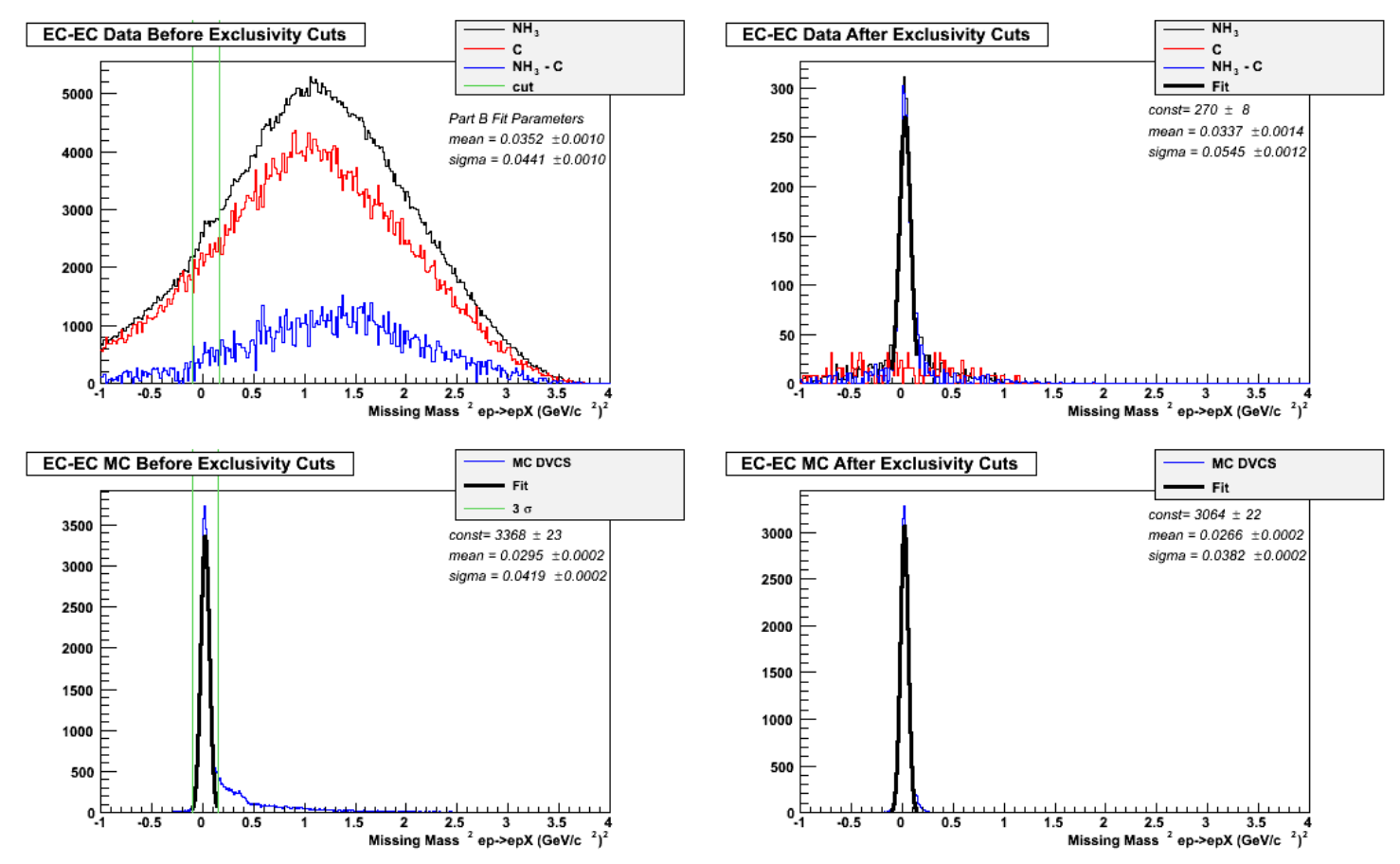

Fig. B.5: Exclusive $\pi^{0}$ analysis Run Period A, EC-EC topology $M M^{2}(e p)$. Top plots: data (black: all $\mathrm{NH}_{3}$ data, red: charge scaled carbon data, blue: $\mathrm{NH}_{3}$ after carbon subtraction); bottom plots: Monte-Carlo simulation for $e p \pi^{0}$. Left plots: without any but the DIS cuts; right plots: with all exclusivity cuts applied but the one on $M M^{2}(e p)$. 

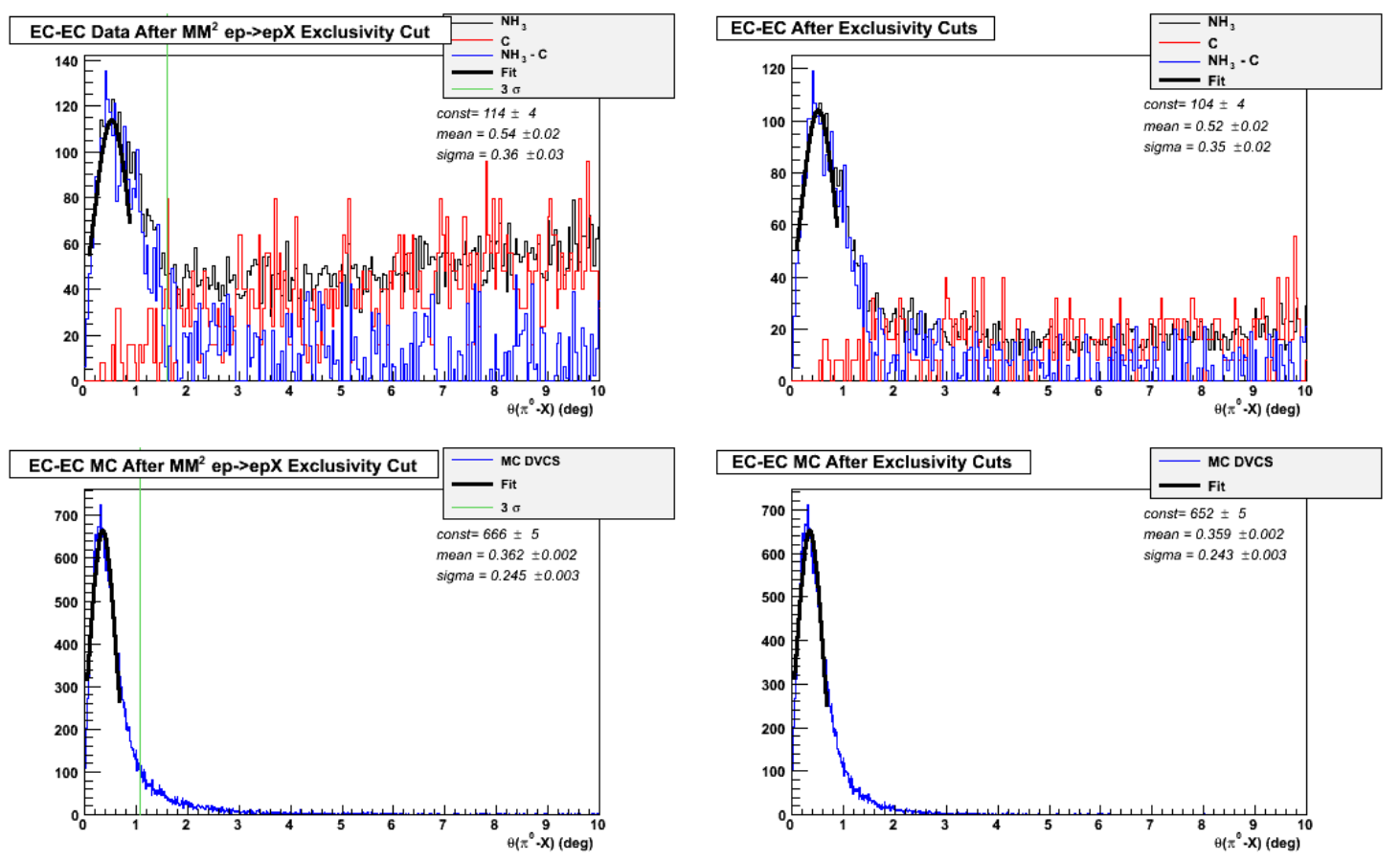

Fig. B.6: Exclusive $\pi^{0}$ analysis Run Period A, EC-EC topology $\theta_{\gamma X}$. Top plots: data (black: all $\mathrm{NH}_{3}$ data, red: charge scaled carbon data, blue: $\mathrm{NH}_{3}$ after carbon subtraction); bottom plots: Monte-Carlo simulation for $e p \pi^{0}$. Left plots: without any but the DIS and the $M M^{2}(e p)$ cuts; right plots: with all exclusivity cuts applied but the one on $\theta_{\pi^{0} X}$. 

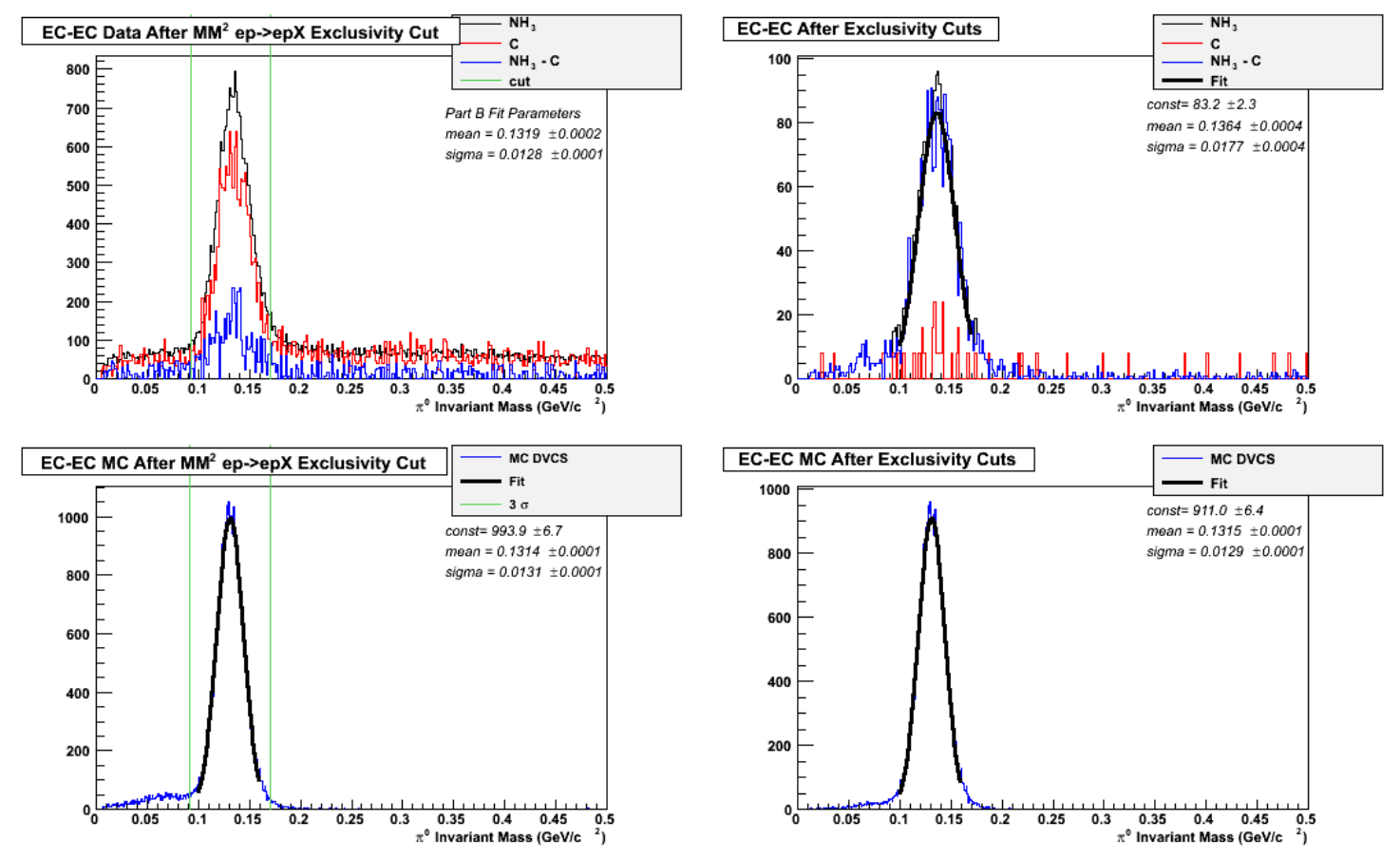

Fig. B.7: Exclusive $\pi^{0}$ analysis Run Period A, EC-EC topology two-photons invariant mass $M(\gamma \gamma)$. Top plots: data (black: all $\mathrm{NH}_{3}$ data, red: charge scaled carbon data, blue: $\mathrm{NH}_{3}$ after carbon subtraction); bottom plots: Monte-Carlo simulation for $e p \pi^{0}$. Left plots: without any but the DIS and the $M M^{2}(e p)$ cuts; right plots: with all exclusivity cuts applied but the one on $M(\gamma \gamma)$. 

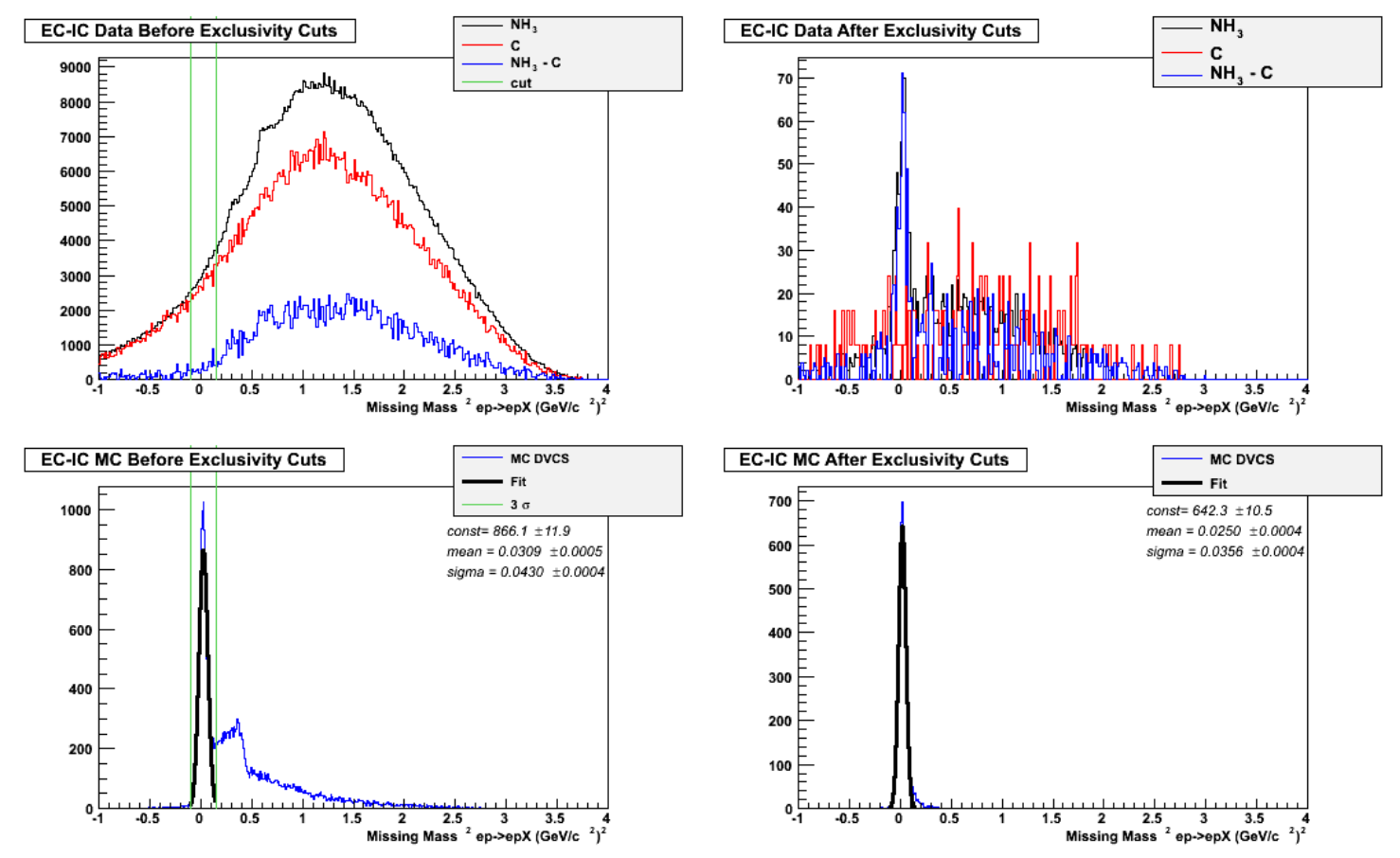

Fig. B.8: Exclusive $\pi^{0}$ analysis Run Period A, EC-IC topology $M M^{2}(e p)$. Top plots: data (black: all $\mathrm{NH}_{3}$ data, red: charge scaled carbon data, blue: $\mathrm{NH}_{3}$ after carbon subtraction); bottom plots: Monte-Carlo simulation for $e p \pi^{0}$. Left plots: without any but the DIS cuts; right plots: with all exclusivity cuts applied but the one on $M M^{2}(e p)$. 

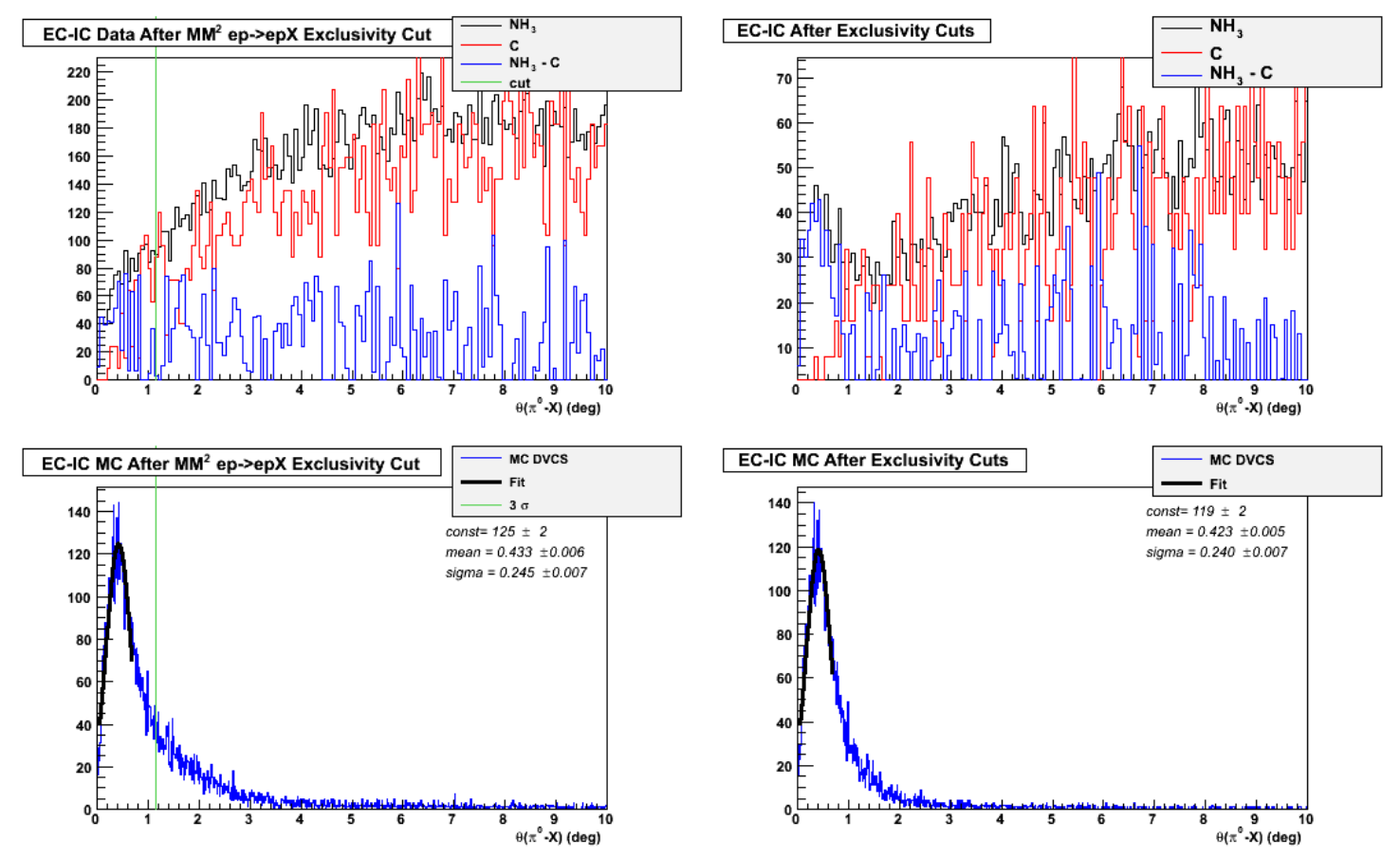

Fig. B.9: Exclusive $\pi^{0}$ analysis Run Period A, EC-IC topology $\theta_{\gamma X}$. Top plots: data (black: all $\mathrm{NH}_{3}$ data, red: charge scaled carbon data, blue: $\mathrm{NH}_{3}$ after carbon subtraction); bottom plots: Monte-Carlo simulation for $e p \pi^{0}$. Left plots: without any but the DIS and the $M M^{2}(e p)$ cuts; right plots: with all exclusivity cuts applied but the one on $\theta_{\pi^{0} X}$. 

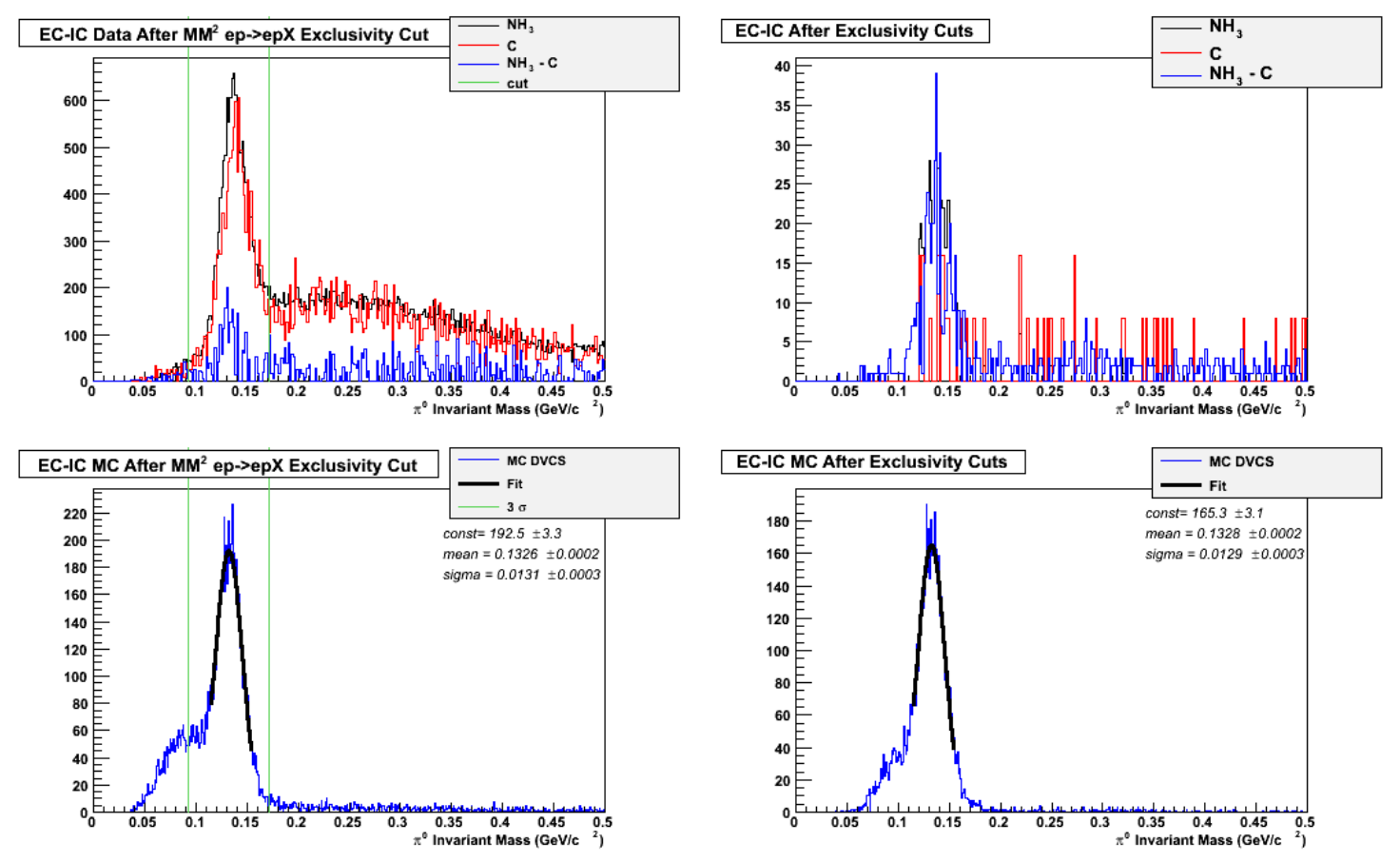

Fig. B.10: Exclusive $\pi^{0}$ analysis Run Period A, EC-IC topology two-photons invariant mass $M(\gamma \gamma)$. Top plots: data (black: all $\mathrm{NH}_{3}$ data, red: charge scaled carbon data, blue: $\mathrm{NH}_{3}$ after carbon subtraction); bottom plots: Monte-Carlo simulation for $e p \pi^{0}$. Left plots: without any but the DIS and the $M M^{2}(e p)$ cuts; right plots: with all exclusivity cuts applied but the one on $M(\gamma \gamma)$. 


\section{Appendix C}

\section{$\pi^{0}$ Contamination - Run Period A}
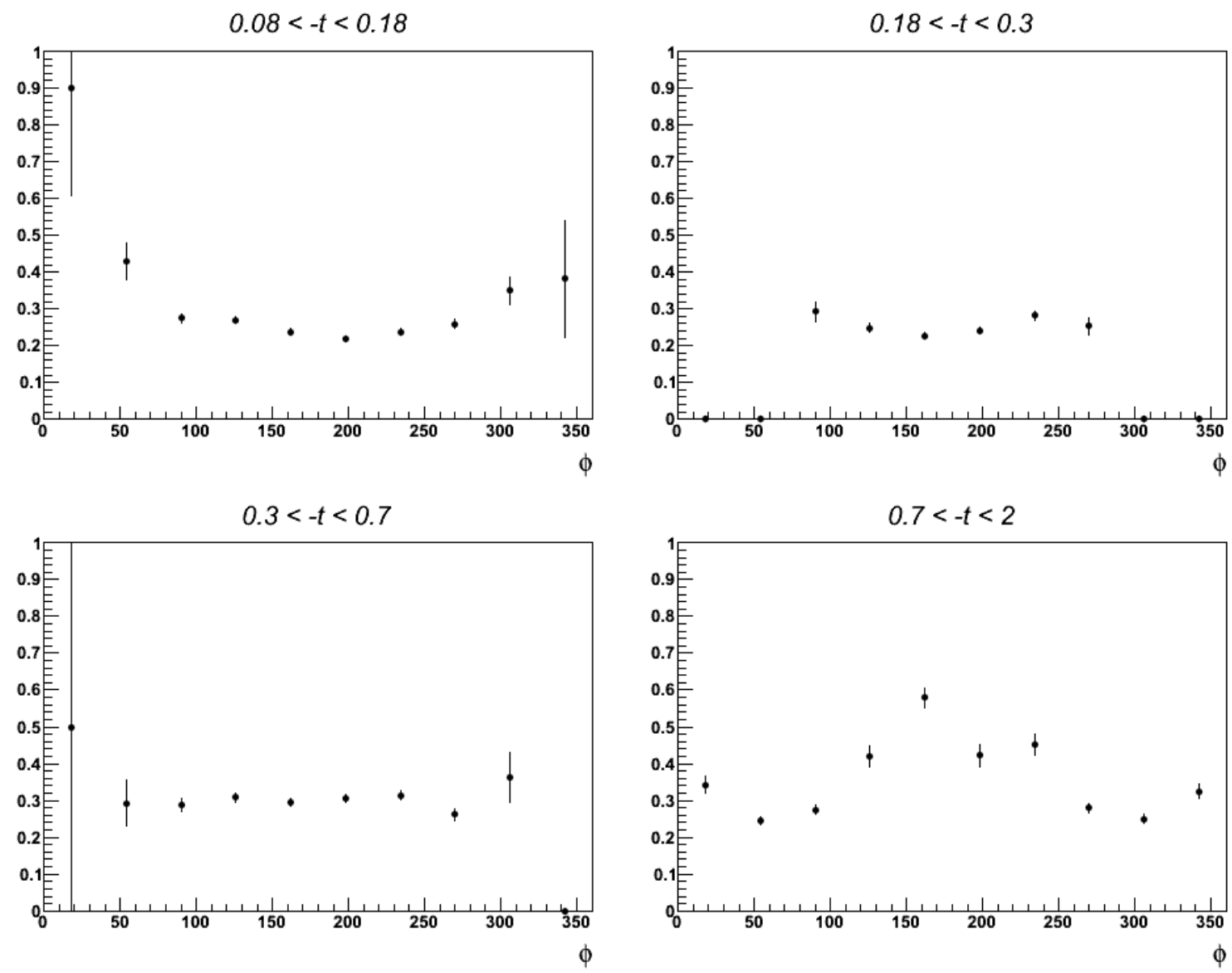

Fig. C.1: Monte-Carlo ratio $\frac{R_{\pi^{0}(\gamma)}}{R_{\pi^{0}(\gamma \gamma)}}$ Run Period A, as a function of $\phi$, for each bin in $-t$, for the first bin in $Q^{2}-x_{B}$. 

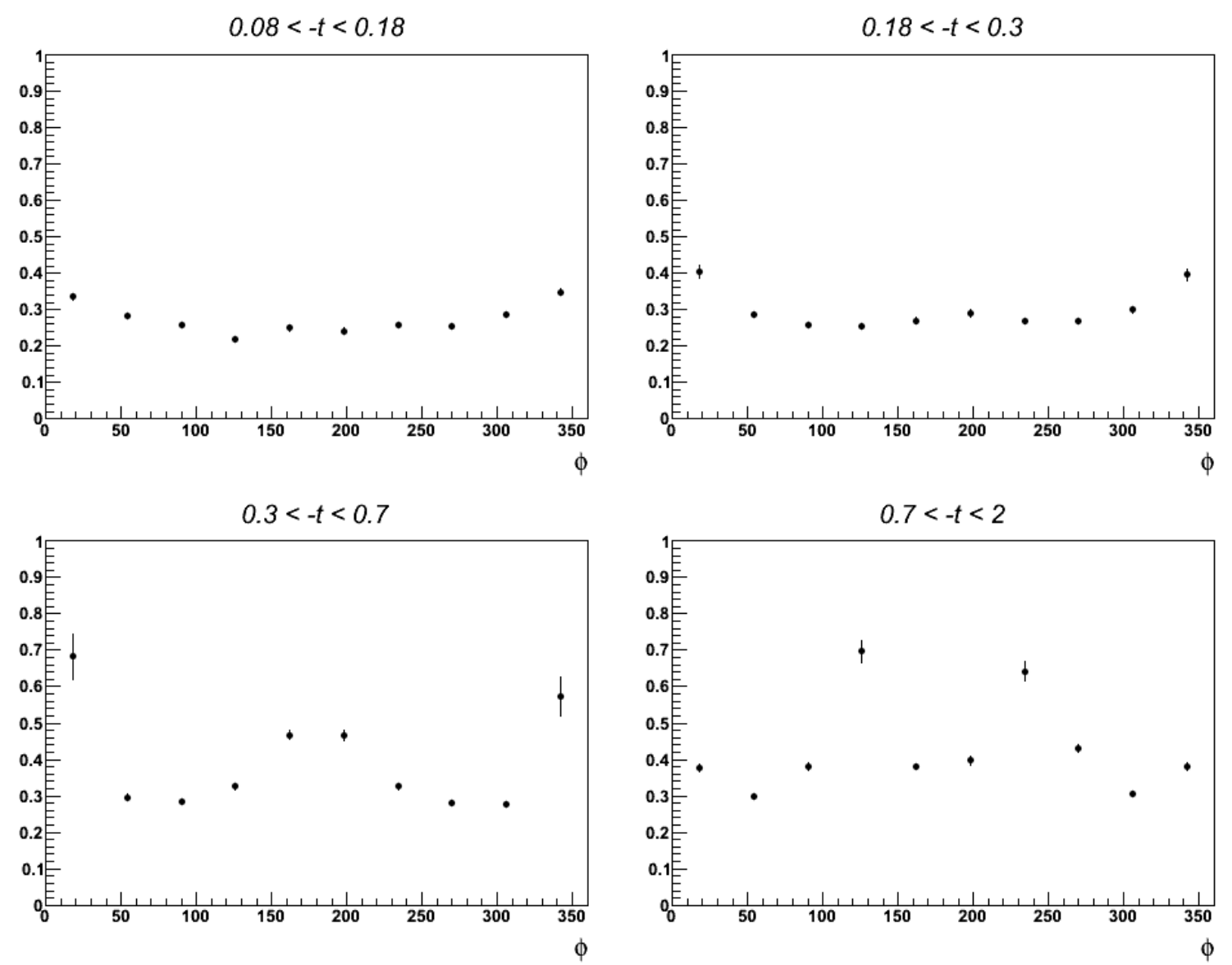

Fig. C.2: Monte-Carlo ratio $\frac{R_{\pi^{0}(\gamma)}}{R_{\pi^{0}(\gamma \gamma)}}$ Run Period A, as a function of $\phi$, for each bin in $-t$, for the second bin in $Q^{2}-x_{B}$. 

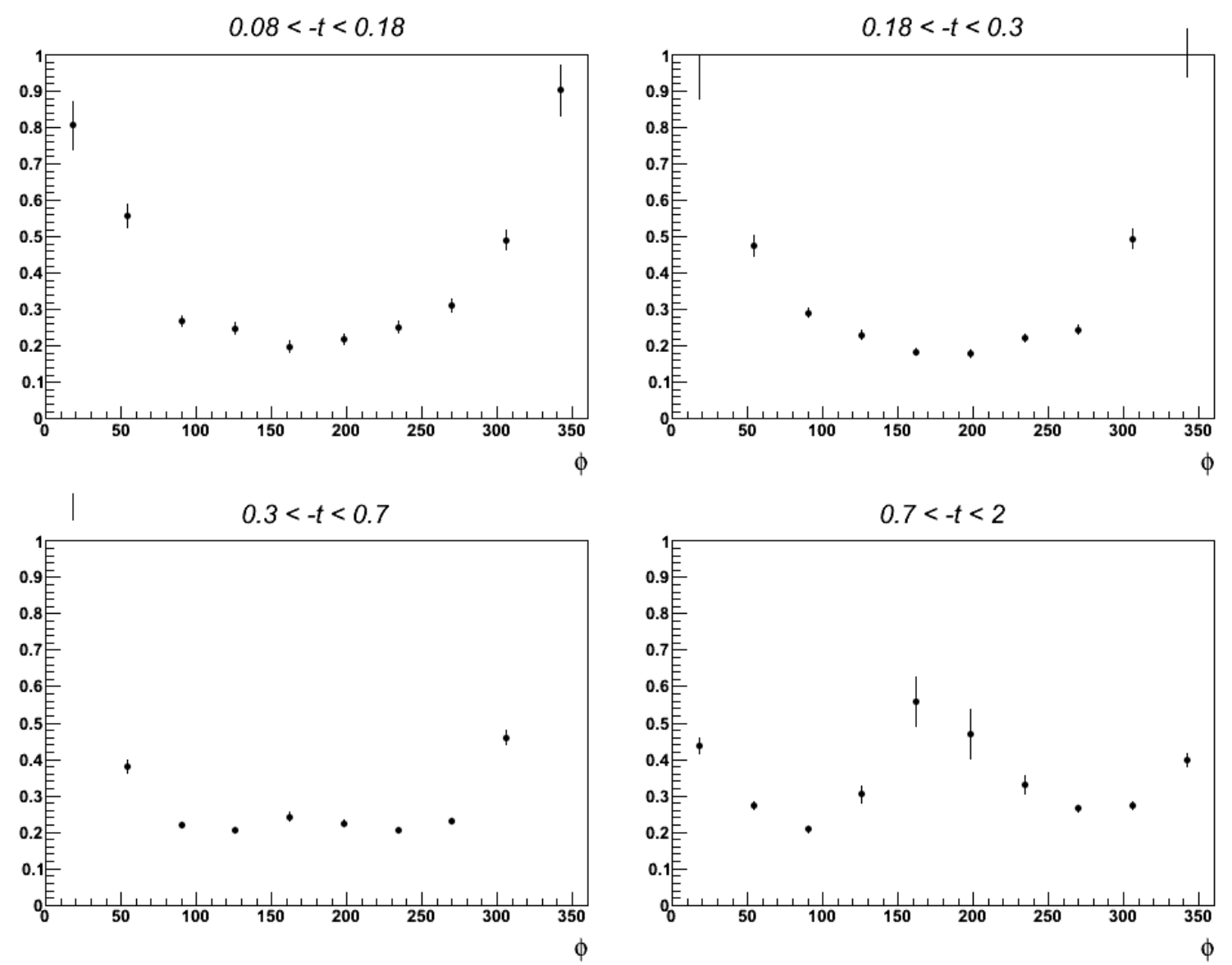

Fig. C.3: Monte-Carlo ratio $\frac{R_{\pi^{0}(\gamma)}}{R_{\pi^{0}(\gamma \gamma)}}$ Run Period A, as a function of $\phi$, for each bin in $-t$, for the third bin in $Q^{2}-x_{B}$. 

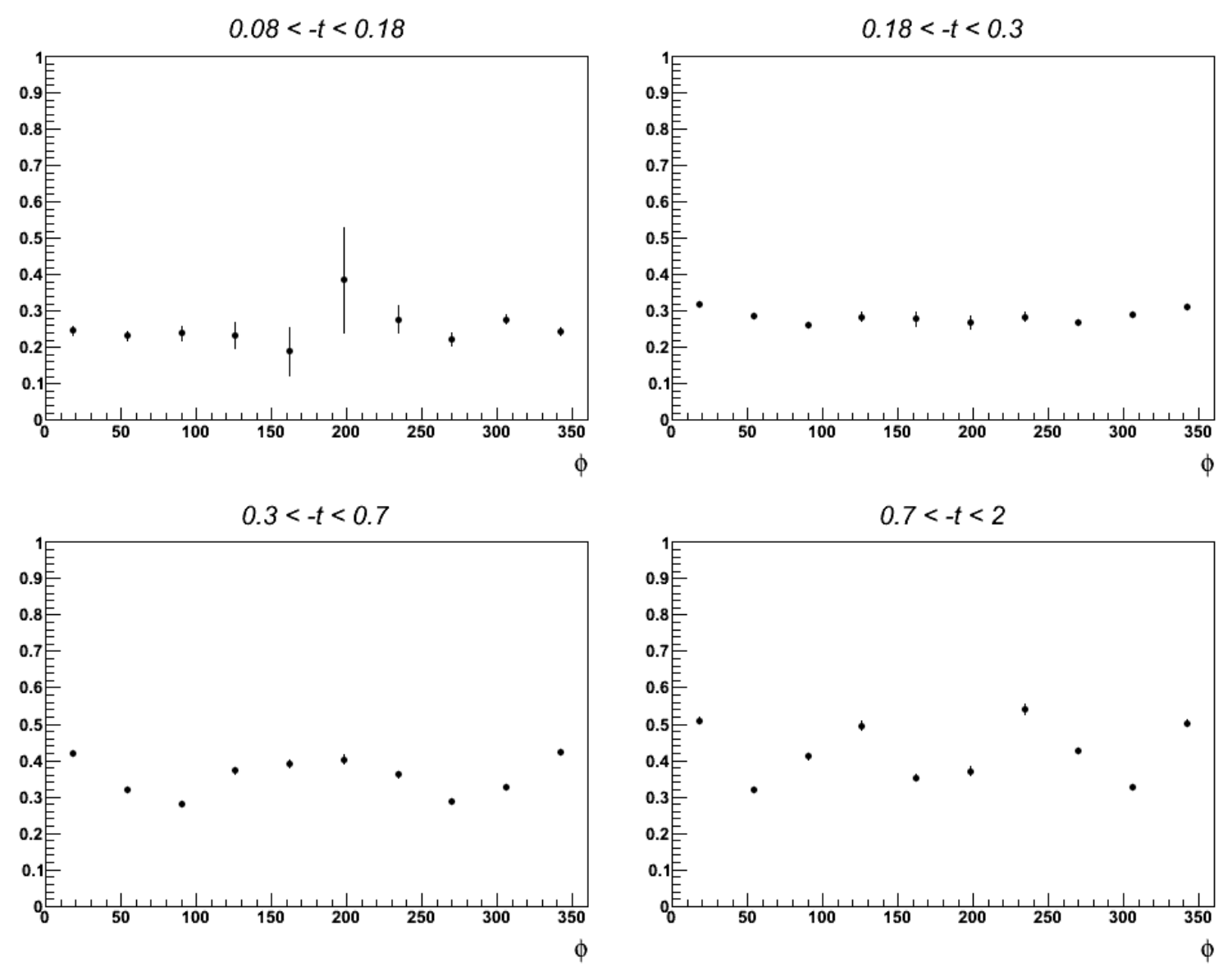

Fig. C.4: Monte-Carlo ratio $\frac{R_{\pi^{0}(\gamma)}}{R_{\pi^{0}(\gamma \gamma)}}$ Run Period A, as a function of $\phi$, for each bin in $-t$, for the fourth bin in $Q^{2}-x_{B}$. 

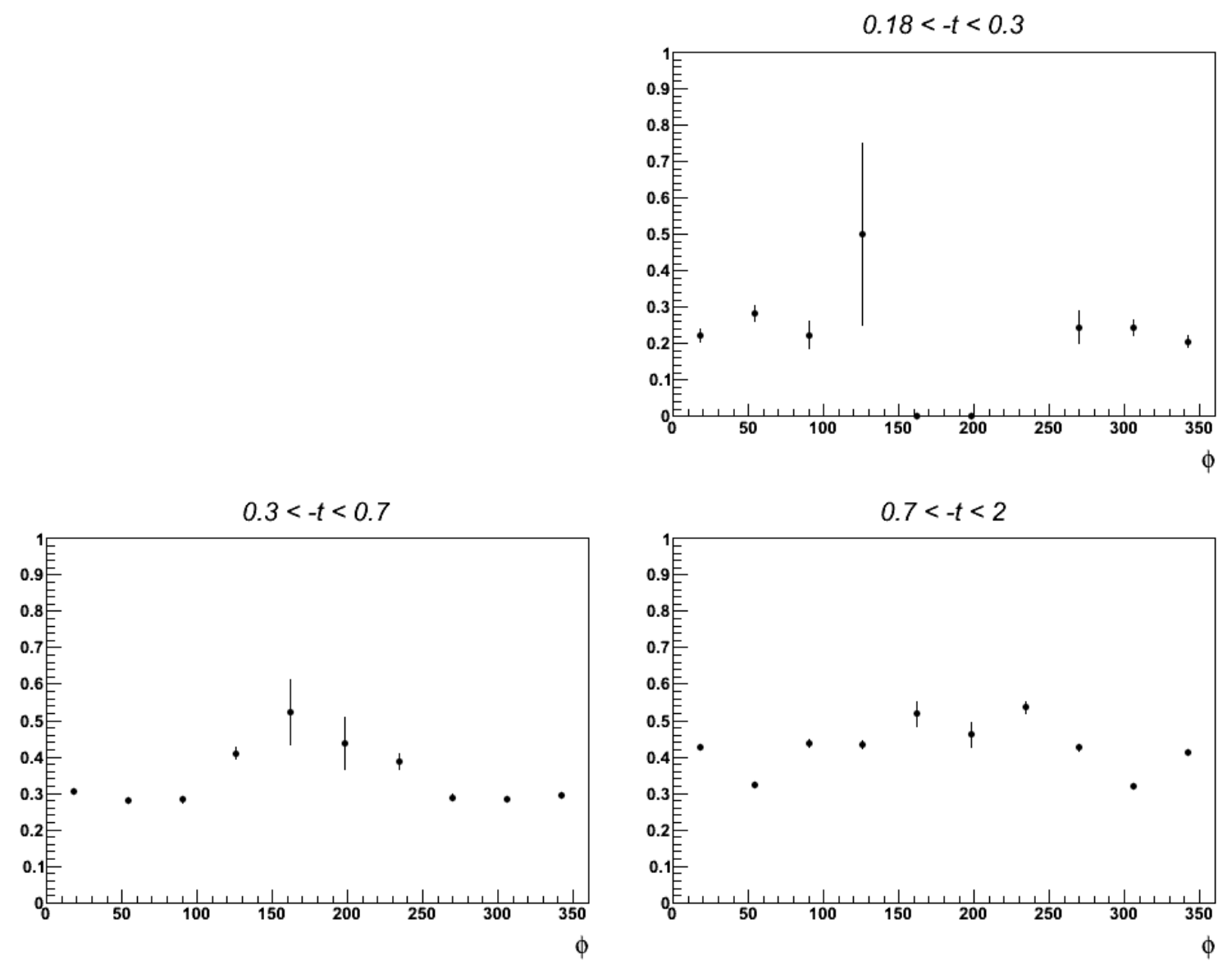

Fig. C.5: Monte-Carlo ratio $\frac{R_{\pi^{0}(\gamma)}}{R_{\pi^{0}(\gamma \gamma)}}$ Run Period A, as a function of $\phi$, for each bin in $-t$, for the fifth bin in $Q^{2}-x_{B}$. 

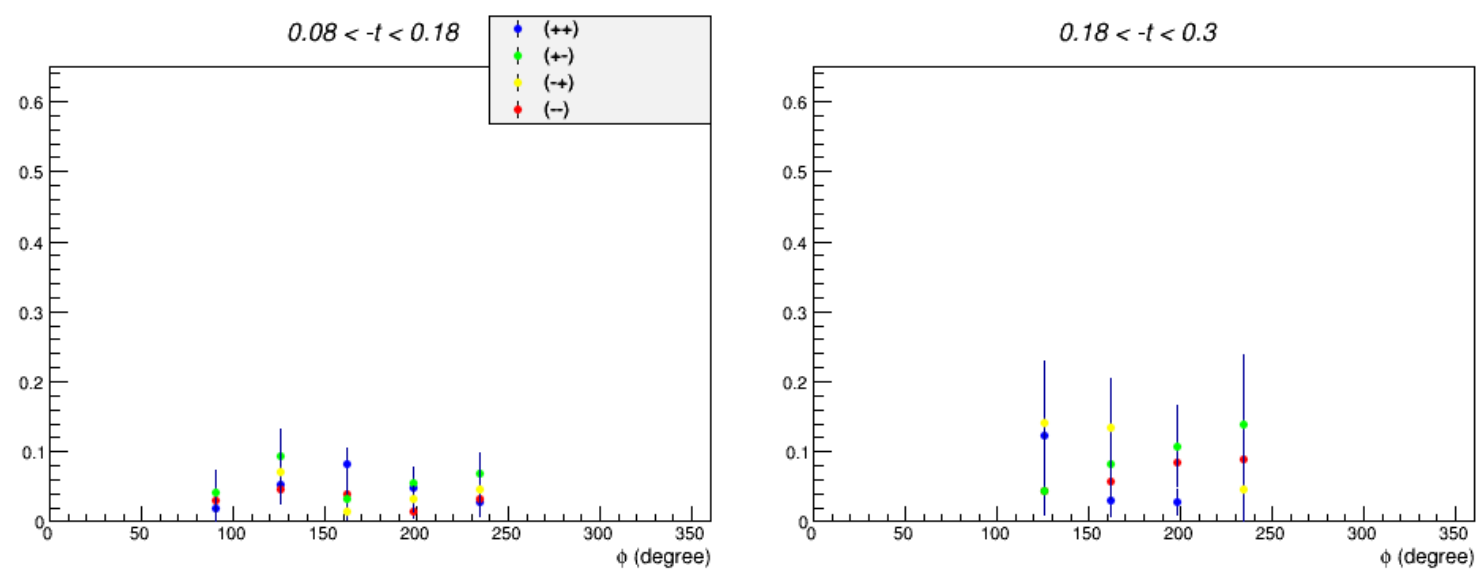

$$
0.3<-t<0.7
$$

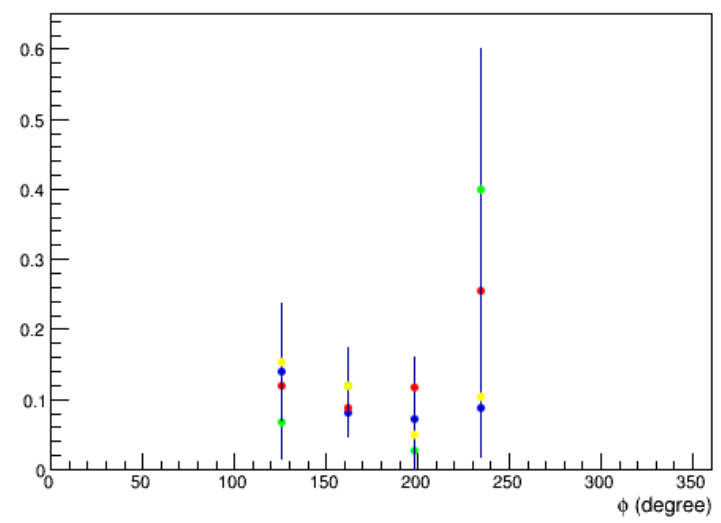

$0.7<-t<2$

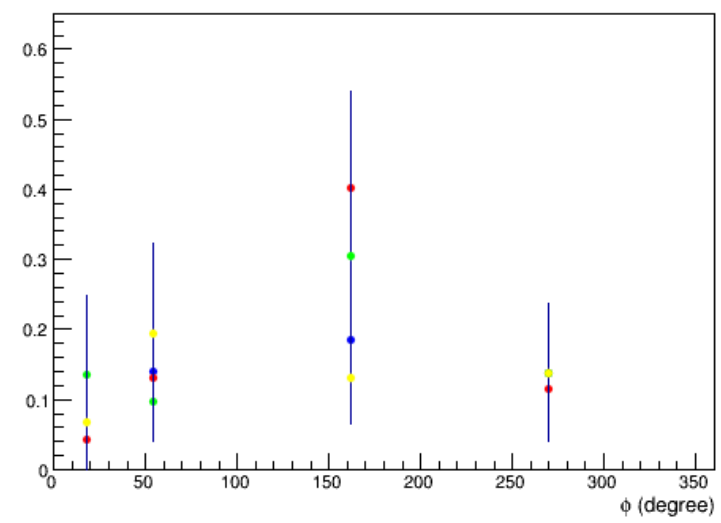

Fig. C.6: Run Period A: Fraction of $\pi^{0}$ contamination over the total number of selected $e p \gamma$ events, $B c k g r_{\pi^{0}}$, as a function of $\phi$, for each bin in $-t$, for the first bin in $Q^{2}-x_{B}$. The color code, where the notation is $b t$, where $b$ is beam helicity, $t$ is target polarization sign, is: ++ blue, -+ red, +- green, -+ yellow. 

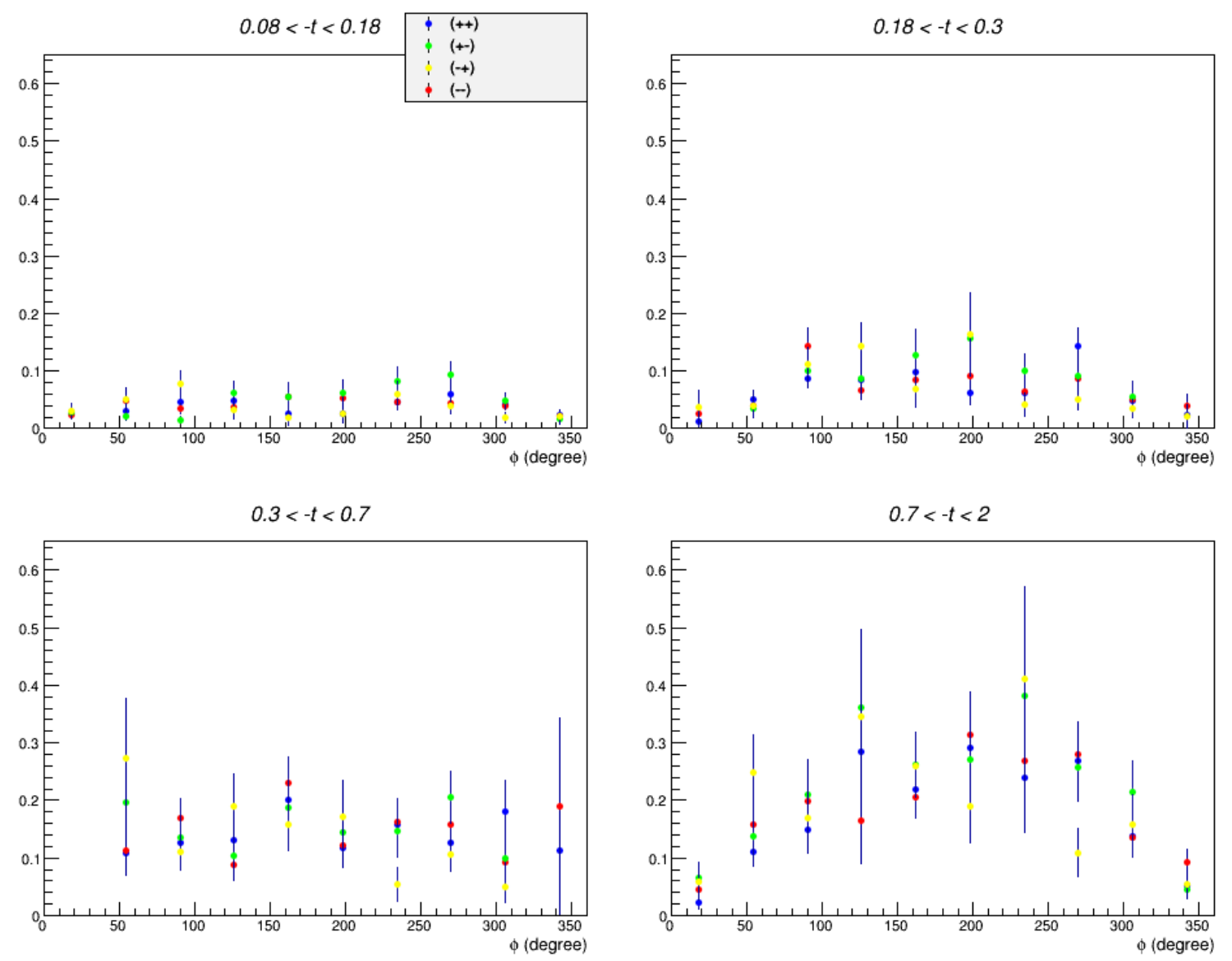

Fig. C.7: Run Period A: Fraction of $\pi^{0}$ contamination over the total number of selected $e p \gamma$ events, $B c k g r_{\pi^{0}}$, as a function of $\phi$, for each bin in $-t$, for the second bin in $Q^{2}-x_{B}$. The color code, where the notation is $b t$, where $b$ is beam helicity, $t$ is target polarization sign, is: ++ blue, -+ red, +- green, -+ yellow. 

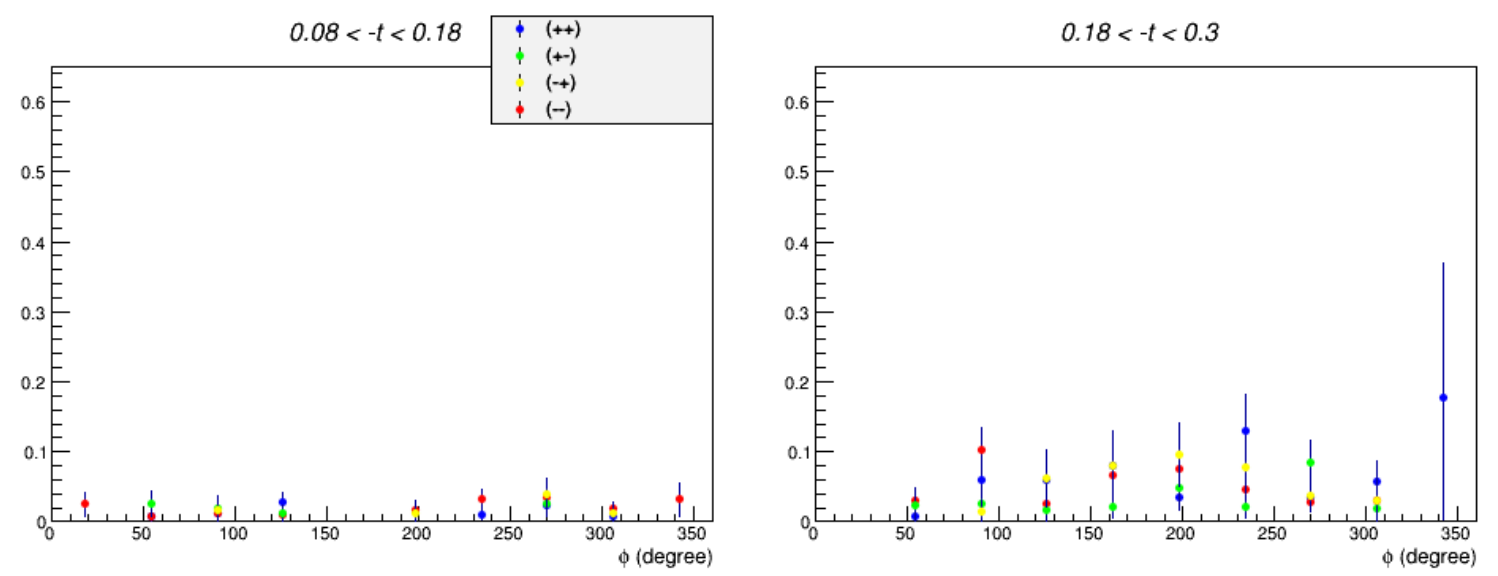

\section{$0.3<-t<0.7$}
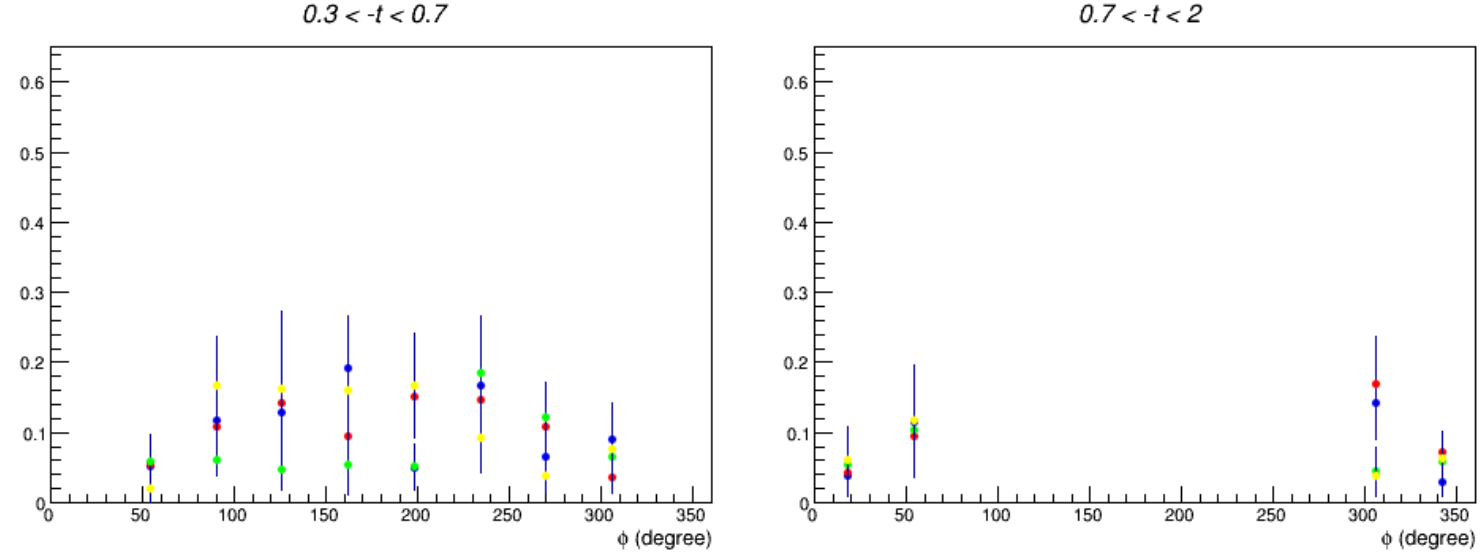

Fig. C.8: Run Period A: Fraction of $\pi^{0}$ contamination over the total number of selected $e p \gamma$ events, $B c k g r_{\pi^{0}}$, as a function of $\phi$, for each bin in $-t$, for the third bin in $Q^{2}-x_{B}$. The color code, where the notation is $b t$, where $b$ is beam helicity, $t$ is target polarization sign, is: ++ blue, -+ red, +- green, -+ yellow. 

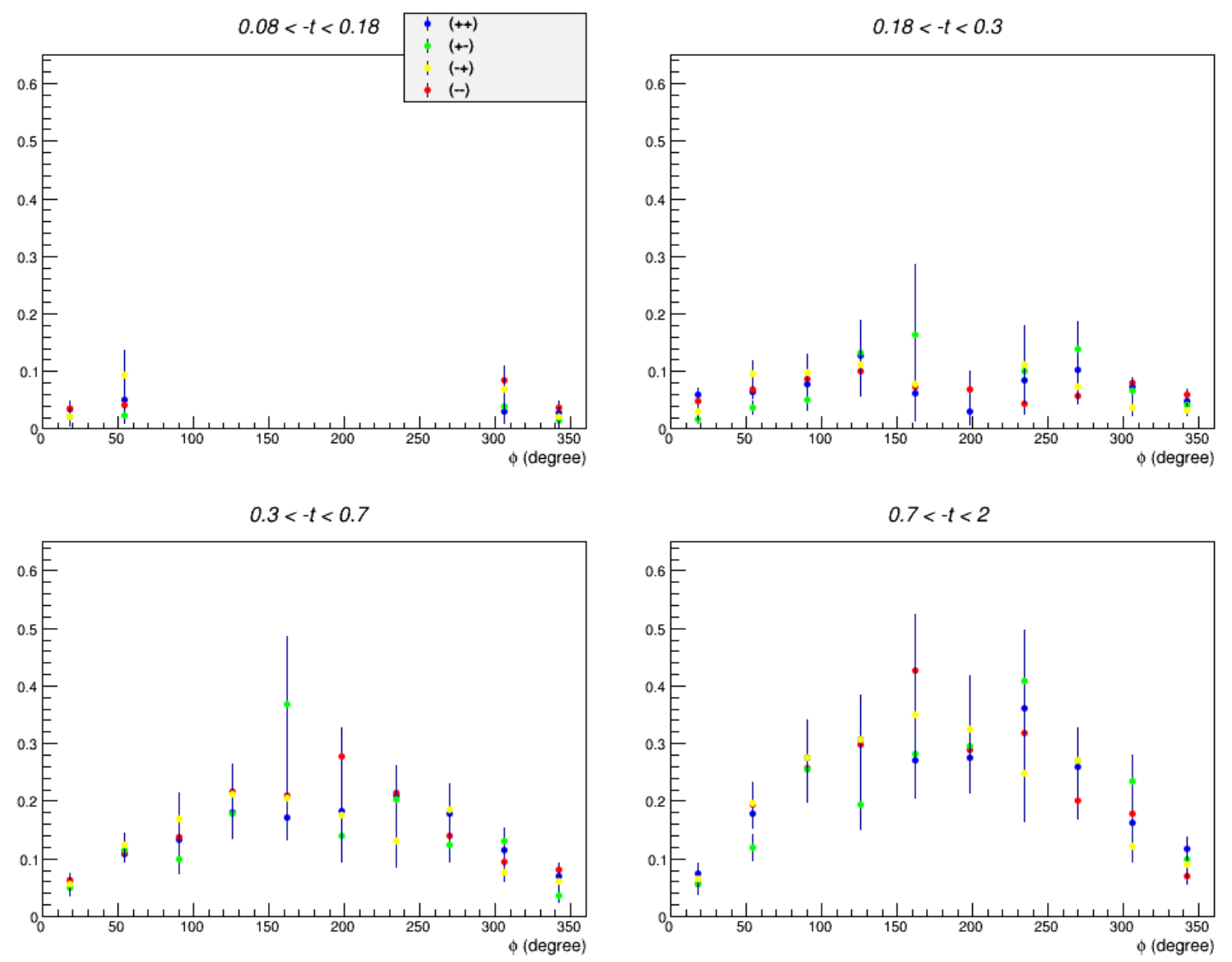

Fig. C.9: Run Period A: Fraction of $\pi^{0}$ contamination over the total number of selected $e p \gamma$ events, $B c k g r_{\pi^{0}}$, as a function of $\phi$, for each bin in $-t$, for the fourth bin in $Q^{2}-x_{B}$. The color code, where the notation is $b t$, where $b$ is beam helicity, $t$ is target polarization sign, is: ++ blue, -+ red, +- green, -+ yellow. 

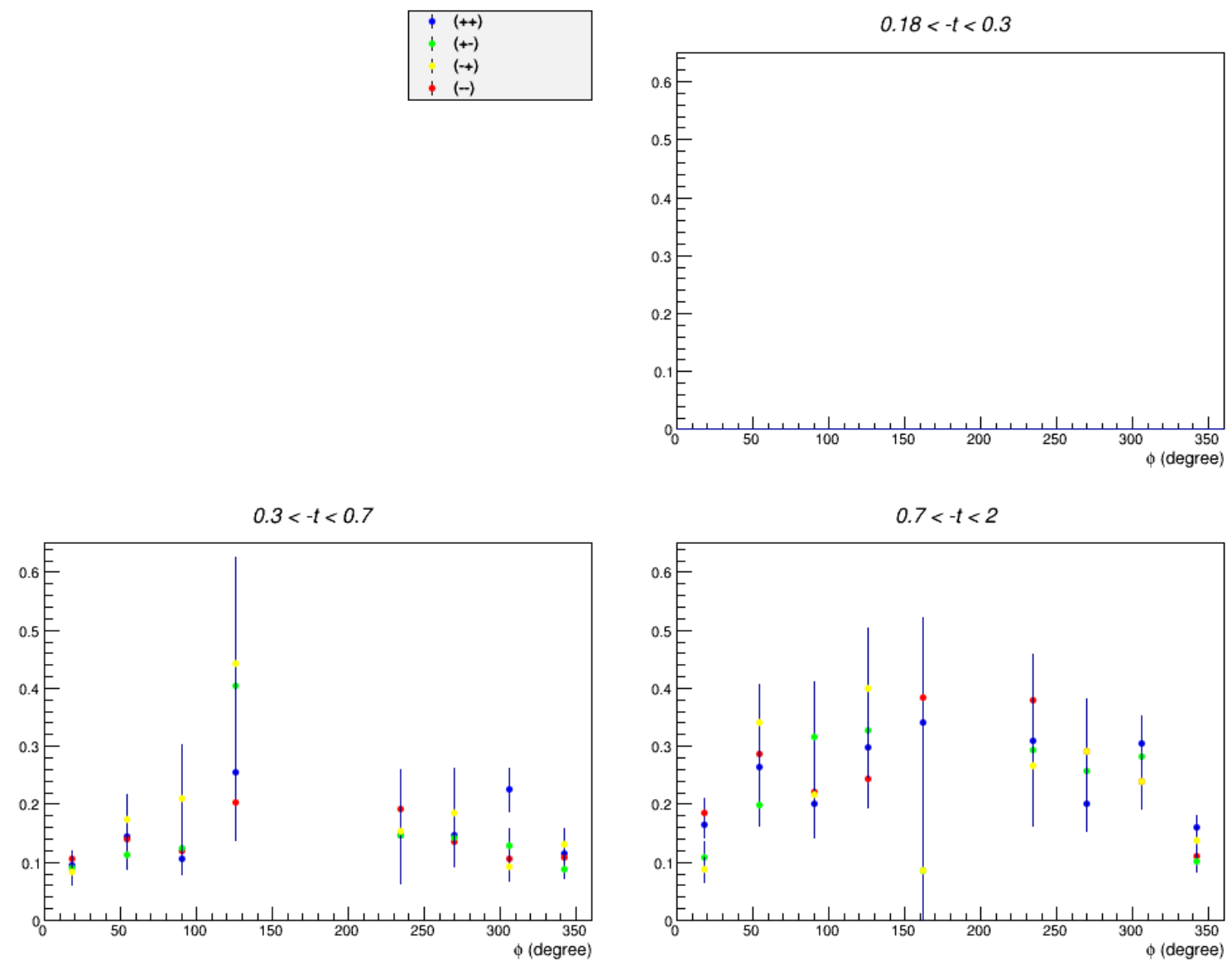

Fig. C.10: Run Period A: Fraction of $\pi^{0}$ contamination over the total number of selected $e p \gamma$ events, $B c k g r_{\pi^{0}}$, as a function of $\phi$, for each bin in $-t$, for the fifth bin in $Q^{2}-x_{B}$. The color code, where the notation is $b t$, where $b$ is beam helicity, $t$ is target polarization sign, is: ++ blue, -+ red, +- green, -+ yellow. 

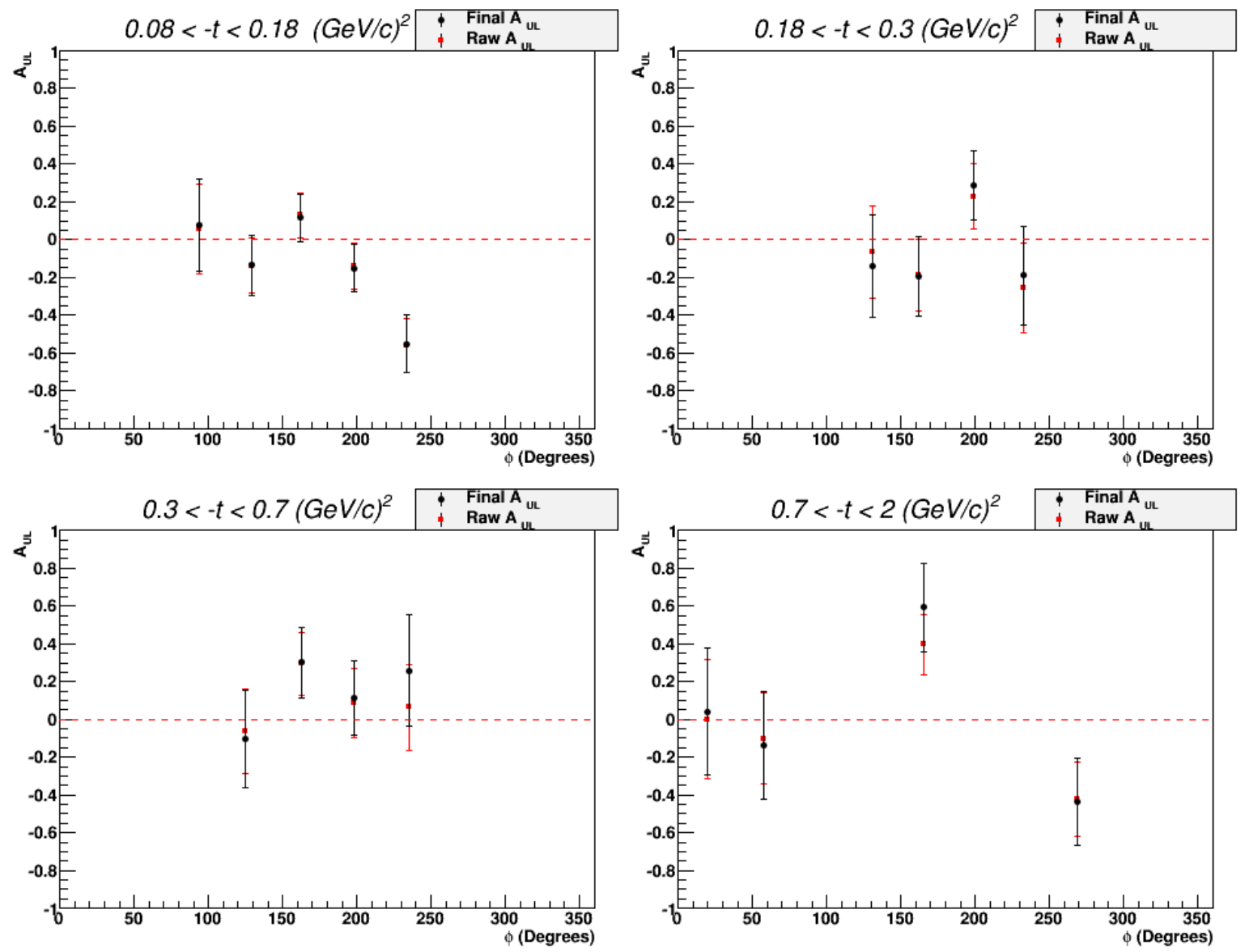

Fig. C.11: Target-spin asymmetry Run Period A, as a function of $\phi$, for each bin in $-t$, and for the first bin in $Q^{2}-x_{B}$ shown for Part B data. The black and red points represent, respectively, the asymmetries including $\pi^{0}$ background subtraction and the raw asymmetries. 

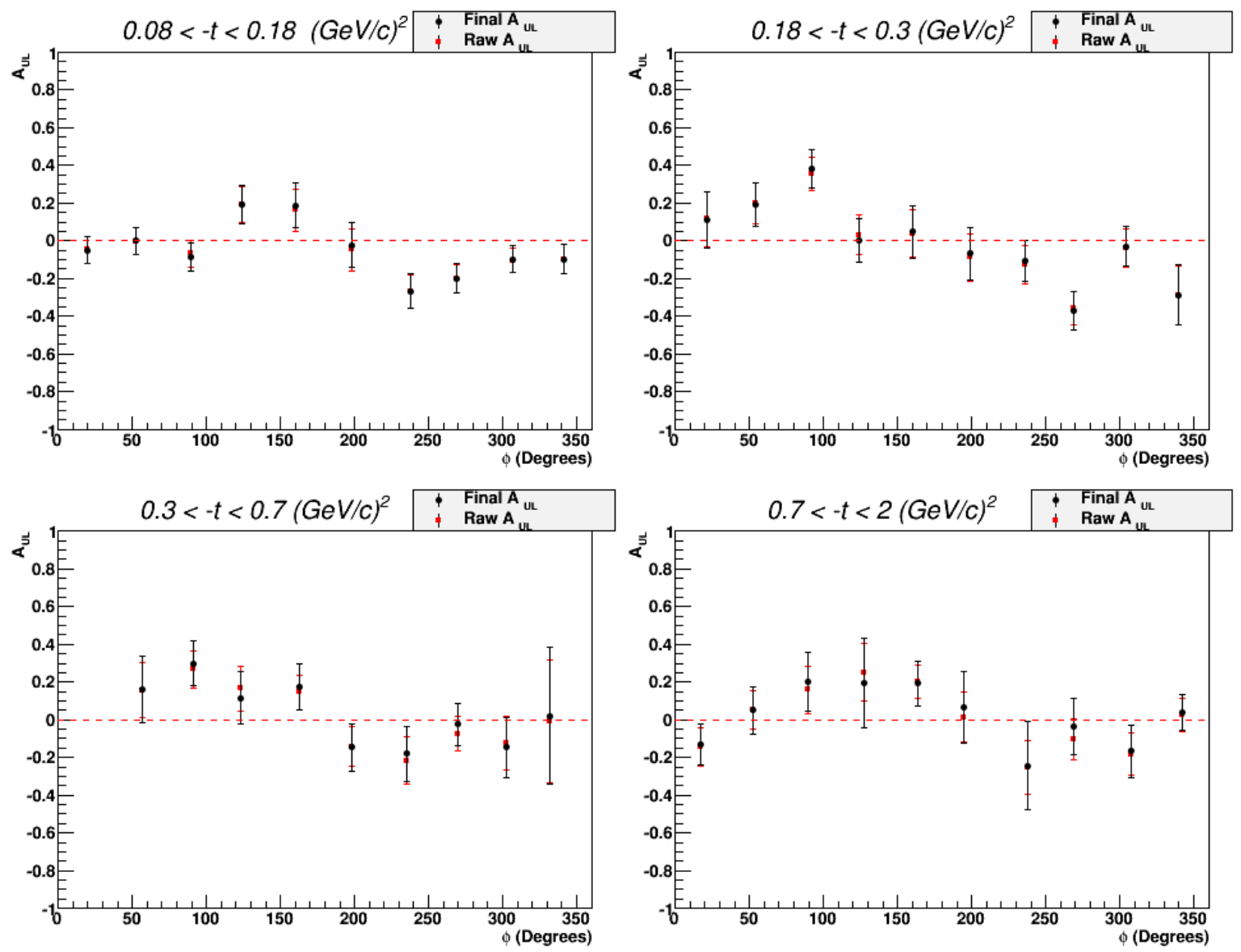

Fig. C.12: Target-spin asymmetry Run Period A, as a function of $\phi$, for each bin in $-t$, and for the second bin in $Q^{2}-x_{B}$ shown for Part B data. The black and red points represent, respectively, the asymmetries including $\pi^{0}$ background subtraction and the raw asymmetries. 

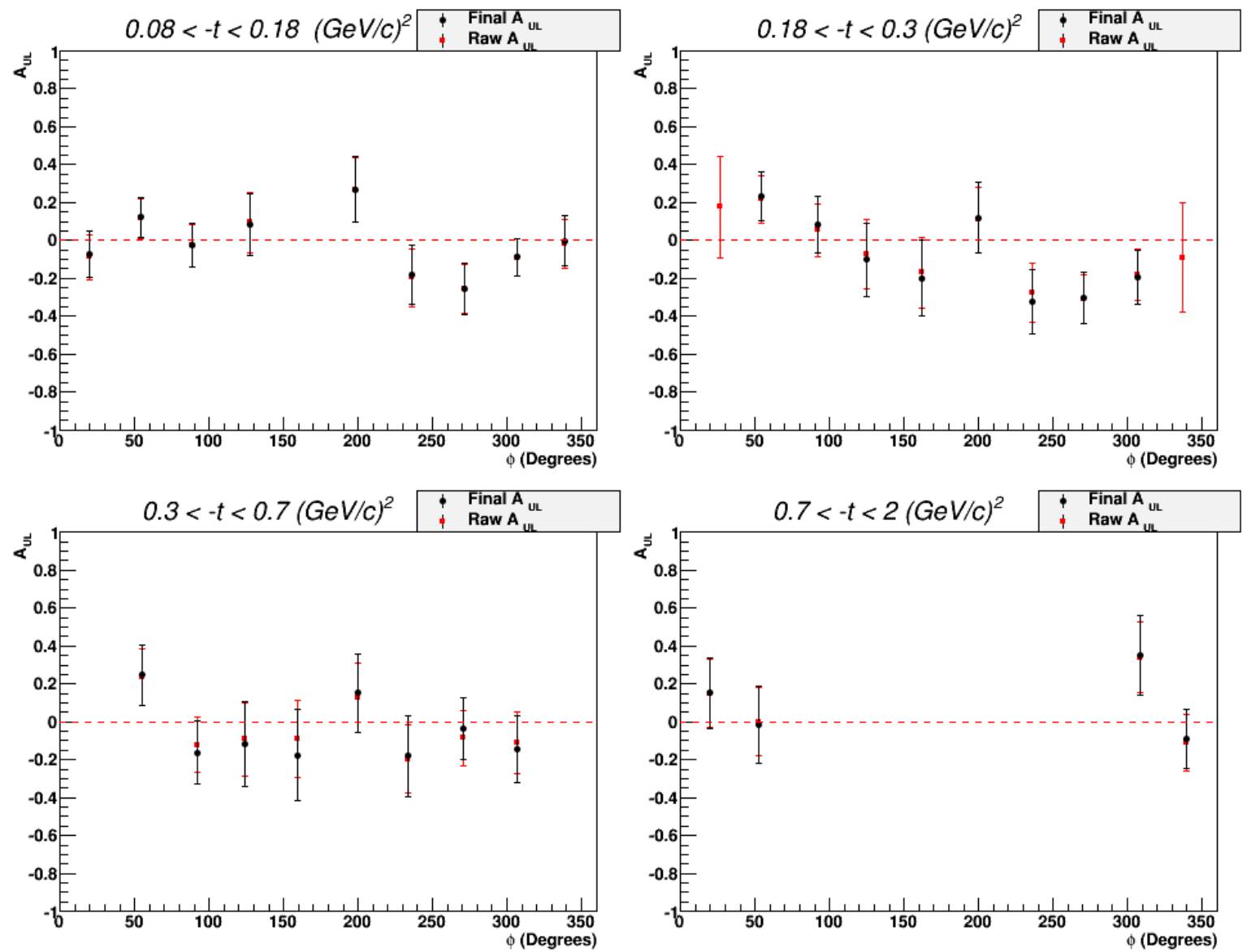

Fig. C.13: Target-spin asymmetry Run Period A, as a function of $\phi$, for each bin in $-t$, and for the third bin in $Q^{2}-x_{B}$ shown for Part B data. The black and red points represent, respectively, the asymmetries including $\pi^{0}$ background subtraction and the raw asymmetries. 

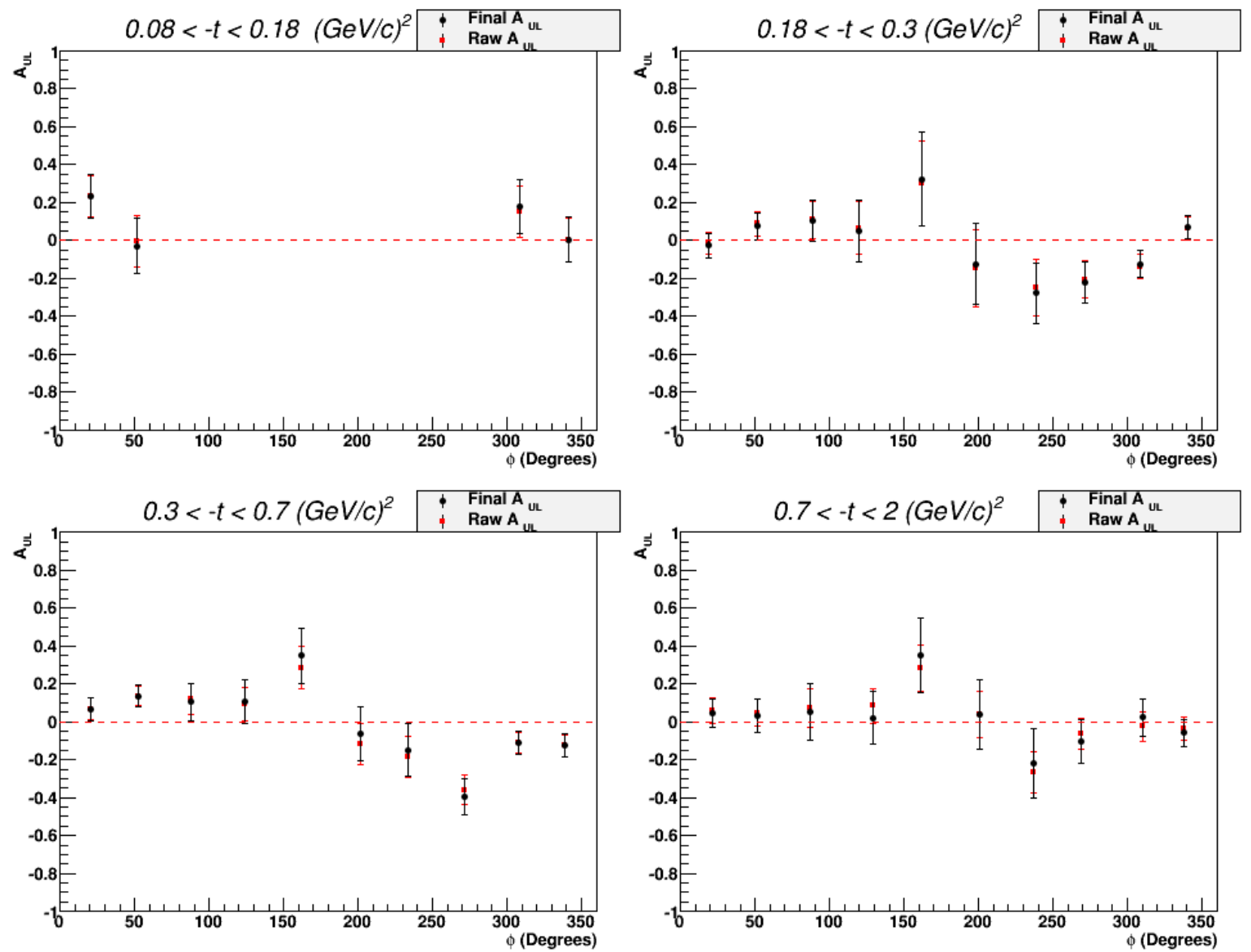

Fig. C.14: Target-spin asymmetry Run Period A, as a function of $\phi$, for each bin in $-t$, and for the fourth bin in $Q^{2}-x_{B}$ shown for Part B data. The black and red points represent, respectively, the asymmetries including $\pi^{0}$ background subtraction and the raw asymmetries. 

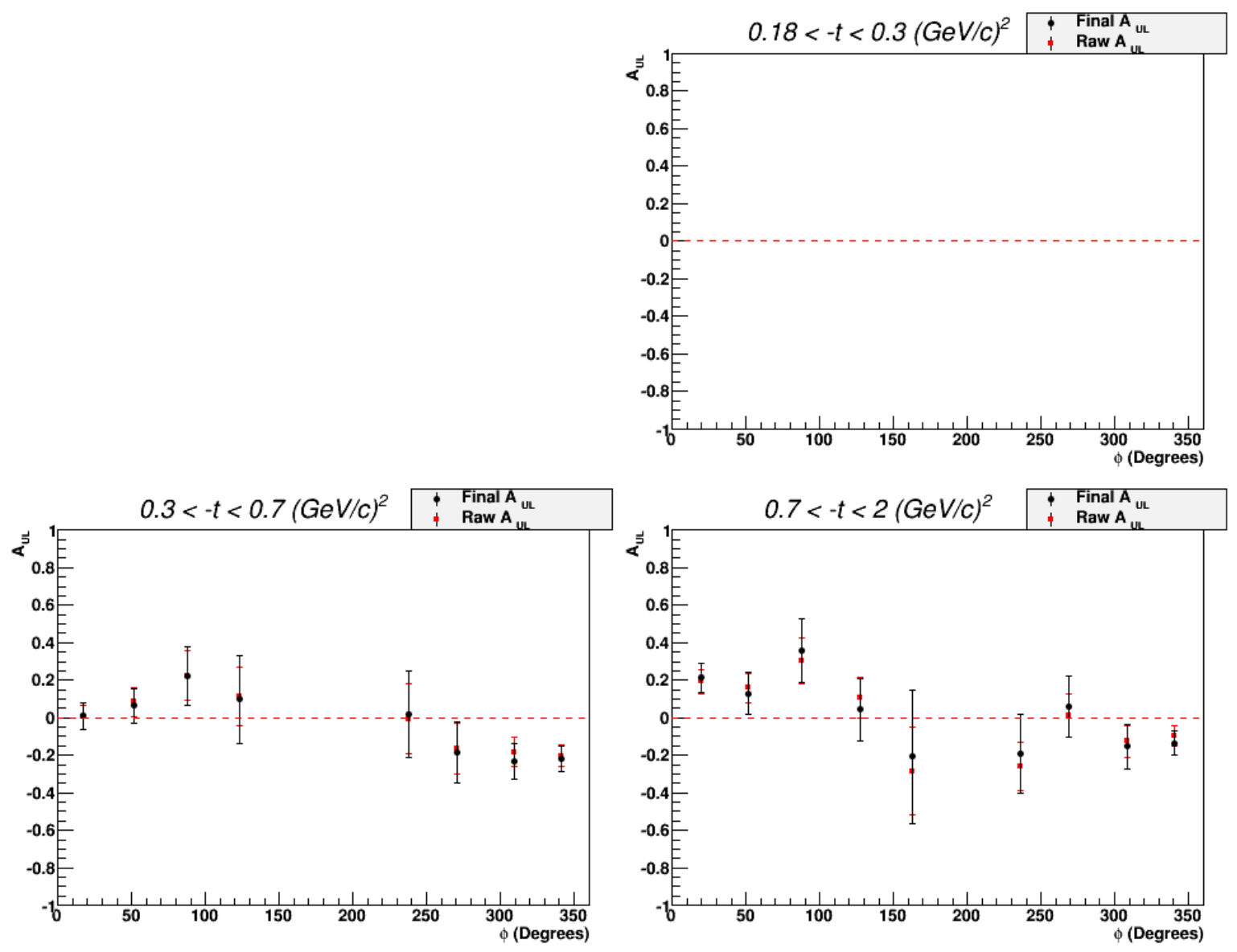

Fig. C.15: Target-spin asymmetry Run Period A, as a function of $\phi$, for each bin in $-t$, and for the fifth bin in $Q^{2}-x_{B}$ shown for Part B data. The black and red points represent, respectively, the asymmetries including $\pi^{0}$ background subtraction and the raw asymmetries. 


\section{Bibliography}

[1] S. Niccolai, S. Pisano, E. Seder, A. Biselli, G. Smith, URL

http : //clasweb.jlab.org/rungroups/eg1 - dves/wiki/index.php/DVCS_analysis_note.

[2] F. Halzen, A.D. Martin Quarks and Leptons. John Wiley \& Sons, Inc. (1984) ISBN $0-471-88741-2$.

[3] M. Guidal, Eur Phys. J. A 37, 319-332 (2008).

[4] M. Guidal, H. Moutarde, M. Vanderhaeghen, Rep. Prog. Phys. 76066202 (2013).

[5] A. Belitsky, D. Muller, and A. Kirchner, Nucl. Phys. B629, 323 (2002).

[6] D. Müller, D. Robaschik, B. Geyer, F.-M. Dittes, and J. Horejsi, Fortschr. Phys. 42 (1994) 101.

[7] X. Ji, Phys. Rev. Lett. 78 (1997) 610; Phys. Rev. D 55 (1997) 7114.

[8] A.V. Radyushkin, Phys. Lett. B 380 (1996) 417; Phys. Rev. D 56 (1997) 5524.

[9] J.C. Collins, L. Frankfurt and M. Strikman, Phys. Rev. D 56 (1997) 2982.

[10] K. Goeke, M. V. Polyakov and M. Vanderhaeghen, Prog. Part. Nucl. Phys. 47 (2001) 401.

[11] M. Diehl, Phys. Rept. 388 (2003) 41.

[12] A.V. Belitsky, A.V. Radyushkin, Phys. Rept. 418 (2005) 1.

[13] M. Burkardt, Phys. Rev. D 62, 071503(2000)

[14] C. Muñoz Camacho et al., Phys. Rev. Lett. 97, 262002 (2006).

[15] F.-X. Girod et al., Phys. Rev. Lett. 100, 162002 (2008).

[16] A. Airapetian et al., arXiv:0802.2499; A. Airapetian et al., JHEP 1006, 019 (2010).

[17] S. Chen et al., Phys. Rev. Lett. 97, 072002 (2006).

[18] B.A. Mecking et al., Nucl. Instr. and Meth. A, 503, 513 (2003).

[19] John O’Meara et al., IEEE TRANSACTIONS ON MAGNETICS, 25, 2, (1989). 
[20] M.D. Mestayer et al., Nucl. Instr. and Meth. A, 449, 81(2000).

[21] G. Adams et al., Nucl. Instr. and Meth. A, 465, 414(2001).

[22] E.S. Smith et al., Nucl. Instr. and Meth. A, 432, 265(1999).

[23] M. Amarian et al., Nucl. Instr. and Meth. A, 460, 239(2001).

[24] J. Pierce, Ph.D. thesis, University of Virginia (2008).

[25] W. Diamond, CEBAF-PR-87-011 (1987)

[26] R. DeVita, Ph.D. thesis, INFN Genova (2001).

[27] P. Bosted, URL http://clasweb.jlab.org/rungroups/eg1 dvcs/wiki/index.php/Result_of_Aug._31_\%22spin_dance\%22.

[28] C. Cuevas et al., Proceedings of the Fifth European Workshop on Beam Diagnostics \& Instrumentation, ESRF, Grenoble, France, (2001), p. 91.

[29] S. Jawalkar and P. E. Bosted, EG1-DVCS Technical Note 007 (2010).

[30] G. Ciullo, M. Contalbrigo, P. Lenisa, Polarized Sources, Targets and Polarimetry: Proceedings of the 13th international Workshop, World Scientific (2011).

[31] C. D. Keith et al., Nucl. Ins. Meth. A 501, 327 (2003).

[32] D. G. Crabb and W.Meyer, Ann. Rev. Nucl. Part. Sci. 47, 67-109 (1997).

[33] G.R. Court, D.W. Gifford, P. Harrison, W.G. Heyes, M.A. Houlden, Nucl. Instr. and Meth. A 324 (1993) 433.

[34] A. Kim, URL http : //clasweb.jlab.org/rungroups/eg1 - dvcs/wiki/index.php/Bad_Files_pass1.

[35] P. Bosted, URL http://clasweb.jlab.org/rungroups/eg1 dvcs/wiki/index.php/Even_newer_IC_shaadow_cut\%2C_and_new_track_quality_cut.

[36] K. S. Egiyan, CLAS NOTE 1999-007.

[37] P. Bosted, URL http : //clasweb.jlab.org/rungroups/eg1 - dvcs/wiki/index.php/Study_of_E/P

[38] W. R. Leo, Techniques for Nuclear and Particle Physics Experiments. Springer Verlag, Berlin and Heidelberg (1987) ISBN 3-540-17386-2.

[39] P. Bosted, EG1-DVCS Technical Note 012 (2010).

[40] F. X. Girod, IC Fiducial Cut, http : //clasweb.jlab.org/rungroups/e1 - dvcs/wiki/index.php/FX's_analysis. 
[41] S. Niccolai, URL

http : //clasweb.jlab.org/rungroups/eg1 - dvcs/wiki/index.php/GPP .

[42] A.V. Belitsky, D. Müller, A. Kirchner, Nucl. Phys. B 629 (2002) 323-392.

[43] I. Bedlinskiy et al., Physical Review Letters 109, 112001 (2012).

[44] M. Garçon and S. Niccolai, private communication.

[45] H.S. Jo, analysis note on DVCS cross section (in progress).

[46] A. Kim, URL http : //clasweb.jlab.org/rungroups/eg1 - dvcs/wiki/index.php/BeamEnergy_@kenjo.

[47] T. W. Donnelly and A. S. Raskin, Annals. of Phys. 169, 247-351 (1986).

[48] P. Bosted, Tech-NOTE E143-19, JLab, (1993).

[49] P. Bosted and S. Jawalkar, EG1-DVCS Technical Note 008 (2010).

[50] P. Bosted and A. Kim, EG1-DVCS Technical Note 002 (2010).

[51] P. Bosted, EG1-DVCS Technical Note 004 (2010).

[52] M. Vanderhaeghen, P.A.M. Guichon, and M. Guidal, Phys. Rev. D 60, 094017 (1999).

[53] M. Guidal, M.V. Polyakov, A.V. Radyushkin and M. Vanderhaeghen, Phys. Rev. D 72,054013 (2005).

[54] F.X. Girod et al., CLAS-analysis note (2007).

[55] S. Chen, Ph.D. thesis, Florida State University (2006).

[56] A.V. Afanasev et al., J. Exp. Theor. Phys. 102, 220 (2006).

[57] S. Niccolai, private communication. 